\title{
Fungal bioactive molecules in interactions with plants and animals
}

\author{
Dissertation \\ for the award of the degree \\ "Doctor rerum naturalium" \\ of Georg-August University Göttingen, Germany \\ within the doctoral program \\ "Plant Responses To Eliminate Critical Threats" \\ of the Georg-August University School of Science (GAUSS)
}

submitted by

\section{Ling Su}

born in Hohhot, China

Göttingen, September 2019 


\section{$\underline{\text { Thesis committee }}$}

Prof. Dr. Petr. Karlovsky, Molecular Phytopathology and Mycotoxin Research, Georg-August University Göttingen, Germany

Prof. Dr. Ivo Feußner, Department of Plant Biochemistry, Albrecht-von-Haller-Institute for Plant Science, Georg-August University Göttingen, Germany

Prof. Dr. Yuelin Zhang, Department of Botany, Faculty of Science, University of British Columbia, Vancouver, Canada

\section{Members of the Examination Board}

Referee: Prof. Dr. Petr. Karlovsky, Molecular Phytopathology and Mycotoxin Research, GeorgAugust University Göttingen, Germany

$2^{\text {nd }}$ Referee: Prof. Dr. Ivo Feußner, Department of Plant Biochemistry, Albrecht-von-HallerInstitute for Plant Science, Georg-August University Göttingen, Germany

\section{Further members of the Examination Board}

Prof. Dr. Yuelin Zhang, Department of Botany, Faculty of Science, University of British Columbia, Vancouver, Canada

Prof. Dr. Gerhard Braus, Department of Molecular Microbiology and Genetics, Georg-August University Göttingen, Germany

Prof. Dr. Andrea Polle, Department of Forest Botany and Tree Physiology, Georg-August University Göttingen, Germany

Prof. Dr. Christiane Gatz, Department of Plant Molecular Biology and Physiology, Albrecht-vonHaller-Institute for Plant Science, Georg-August University Göttingen, Germany

Date of disputation: $15^{\text {th }}$ October 2019 


\section{Contents}

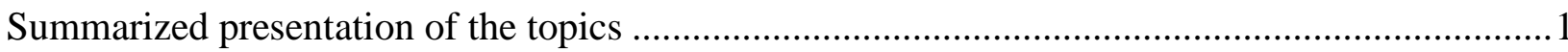

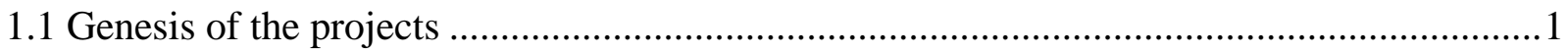

1.2 Stereotypes in the research on NLPs and phytoalexins may prevent us from asking

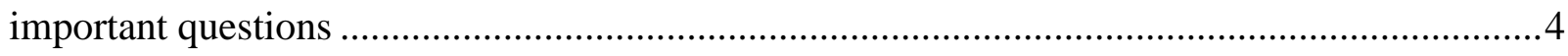

1.3 "Ecological molecules" as a common theme .........................................................

1.4 Ecological molecules: proteins or metabolites? ...................................................

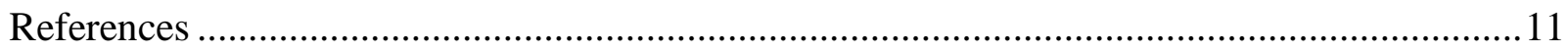

Article I: Elucidation of biological roles of NLPs of Verticillium longisporum in interaction with

Brassica napus and fungivorous invertebrates................................................................ 13

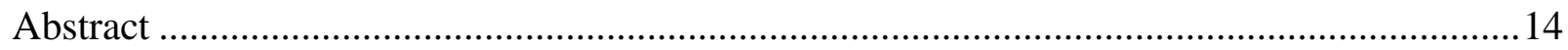

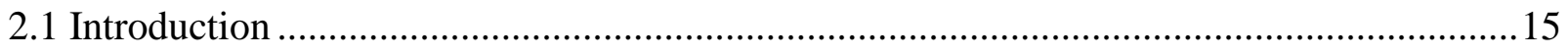

2.1.1 The fungal genus Verticillium ................................................................ 15

2.1.2 Necrosis and ethylene-inducing peptide 1 (Nep 1)-like proteins (NLPs) ................... 19

2.1.3 Cytotoxicity of NLPs and their activity as MAMPs ...........................................22

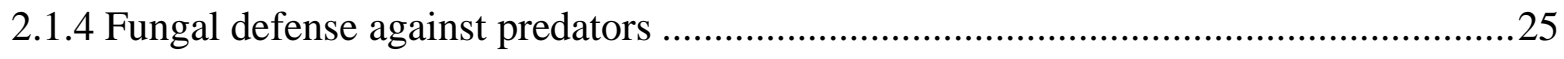

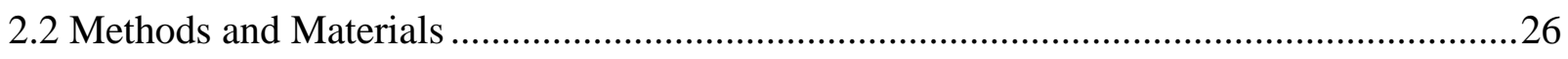

2.2.1 Bioinformatic analysis of VINLPs and their genes ...........................................26

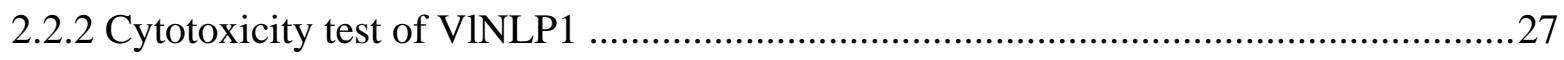

2.2.3 Activation of Mitogen-activated protein kinases (MAPK) ...................................28

2.2.4 V. longisporum strains, culture conditions and RNA extraction..............................29

2.2.5 Vector construction and Agrobacterium-mediated transformation.............................30

2.2.6 Testing $V$. longisporum strains in plants .......................................................... 31

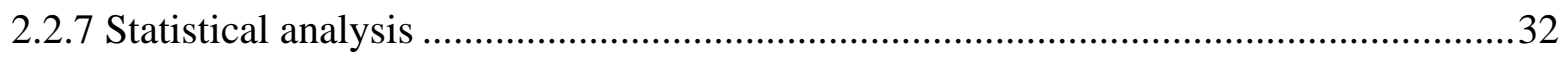

2.2.8 Effects of NLPs on fungal predation ............................................................ 32 


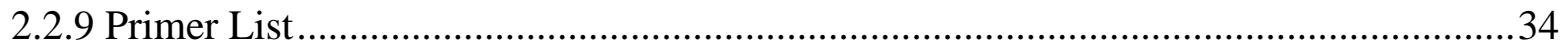

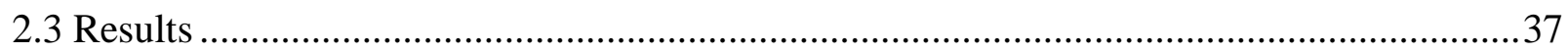

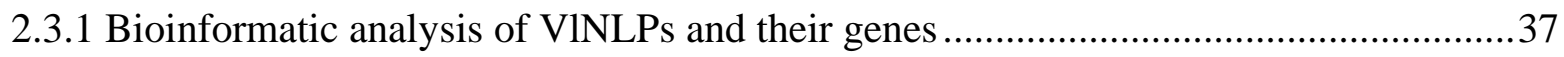

2.3.2 Necrosis-inducing activity of VINLP1 .............................................................43

2.3.3 Plant immune responses caused by VINLP1 ...................................................45

2.3.4 VlNLPs expression in various Verticillium isolates ...............................................48

2.3.5 Overexpression of VINLP3 and VINLP2 in $V$. longisporum VL43 _........................52

2.3.6 Do NLPs protect filamentous fungi from predation? ...........................................57

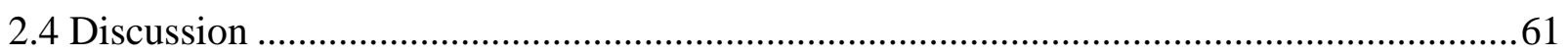

2.4.1 Comparison of NLPs from $V$. dahliae and $V$. longisporum ...................................61

2.4.2 Necrosis-inducing activity of NLPs ..............................................................62

2.4.3 Activation of plant immunity-associated responses by NLPs................................62

2.4.4 Primary biological function of NLPs is unlikely related to the virulence of plant

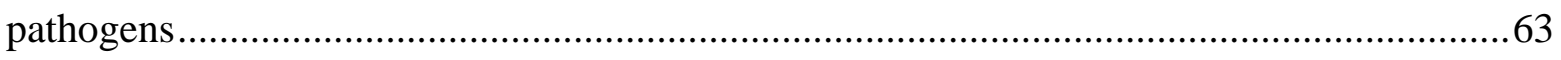

2.4.5 Biological function of NLPs in defense against predation was not supported............66

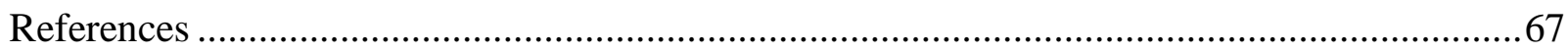

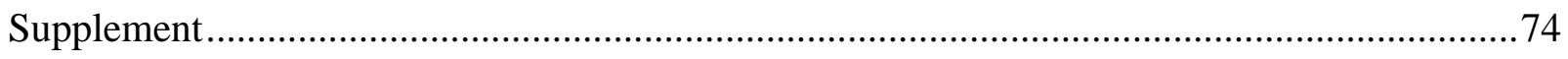

Article II: Induction of phytoalexins synthesis in oilseed rape by Verticillium longisporum and

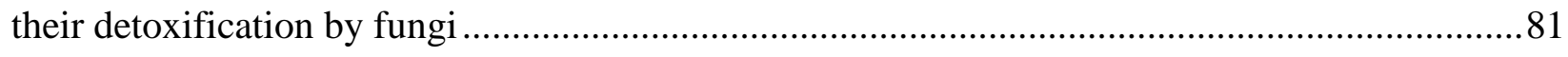

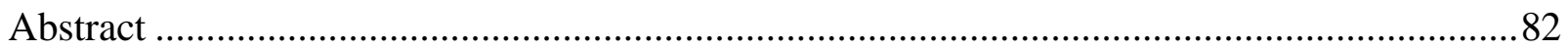

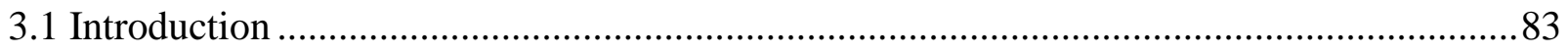

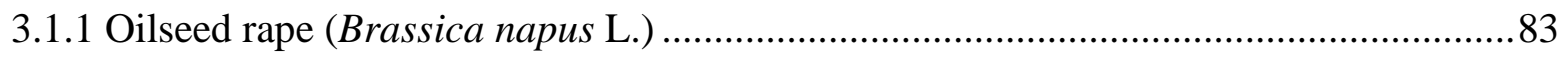

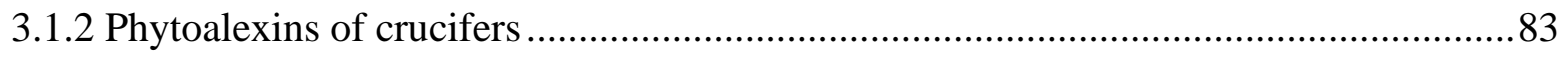

3.1.3 Detoxification of phytoalexins by pathogens ............................................... 84

3.1.4 Degradation of phytoalexins and fungal virulence ........................................... 85

3.1.5 Metabolic profiling of xylem sap from oilseed rape infected by $V$. longisporum.........87 


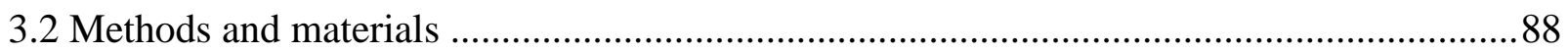

3.2.1 Chemicals, plant material and fungal strains ................................................... 88

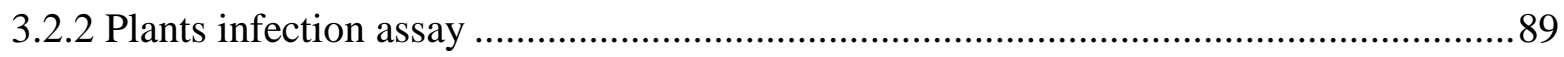

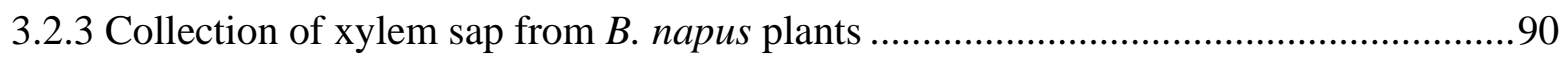

3.2.4 Abiotic and biotic elicitation of phytoalexins in B. napus .................................... 90

3.2.5 Extraction of secondary metabolites ........................................................... 90

3.2.6 Degradation of phytoalexins by fungi ....................................................... 91

3.2.7 Analysis of phytoalexins and their degradation products by HPLC .........................91

3.2.8 Quantification of fungal biomass ............................................................... 92

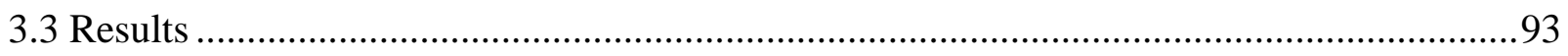

3.3.1 Phytoalexins in xylem sap of oilseed rape plants infected with $V$. longisporum ..........93

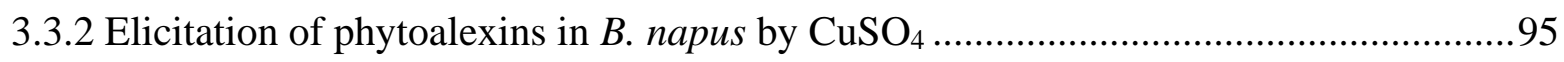

3.3.3 Brassilexin degradation in fungal cultures ......................................................96

3.3.4 Brassinin degradation by fungal cultures .................................................... 103

3.3.5 Cyclobrassinin degradation in fungal cultures .............................................. 106

3.3.6 Brassilexin inhibit germination of $V$. longisporum spores ..................................... 109

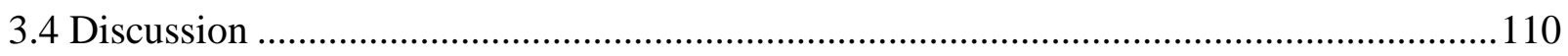

3.4.1 Elicitation of phytoalexins in B. napus by $V$. longisporum infection .......................111

3.4.2 Degradation of phytoalexins by Verticillium spp............................................. 114

3.4.3 Toxicity of phytoalexins to $V$. longisporum .................................................... 115

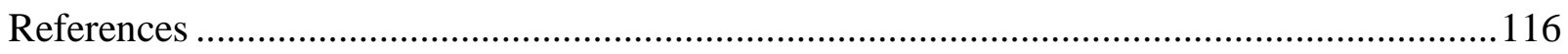

Article III: Bis-naphthopyrone pigments protect filamentous ascomycetes from a wide range of predators

Article IV: Small-Scale Bioreactor for Sterile Hydroponics and Hairy Roots: Metabolic Diversity and Salicylic Acid Exudation by Hairy Roots of Hyoscyamus niger .......................................140

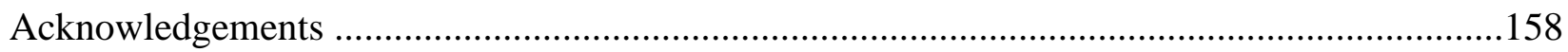




\section{Summarized presentation of the topics}

\subsection{Genesis of the projects}

This thesis covers four topics. In three topics, the working hypotheses were based on the assumption that bioactive molecules modulate interactions of fungi with plants or animals in certain ways. The molecules supposed to mediate fungal interactions in the first three projects were (i) fungal proteins hypothesized to modulate infection of host plants and/or protect the producers of the proteins from predators; (ii) plant metabolites toxic to microorganisms, which are supposed to prevent infection of their producers with pathogens; and (iii) fungal metabolites supposed to deter animal predators. The fourth project was unrelated to the common theme. I became part of my thesis as I supported a fellow student who lacked skills in sequence analysis and data processing, which I acquired while studying certain genes in Verticillium longisporum (see below). Some results obtained in the last project, however, tuned out to be relevant for general considerations about ecological roles of biologically active molecules, which were inspired by first three projects. In the following, the genesis of the projects is described and and decisions made regarding the focus and ramifications of the work and exploitation of new ideas are explained.

In the first project, the bioactive molecules studied were NLPs (Necrosis and Ethylene Inducing Peptide 1-Like Proteins) produced by the pathogen of crucifers Verticillium longisporum. The proposal for this project was based on preliminary results indicating that $V$. longisporum inherited a rather large set of $N L P$ genes from its parents and that some of these genes appeared not to be expressed. A second premise for the proposal was the observation that NLPs from many microbes act as microbe-associated molecular patterns (MAMPs), triggering plant defense responses (Oome et al. 2014). The proposal was based on the hypothesized that some NLPs of $V$. longisporum trigger immune response in B. napus, and that the genes encoding these NLPs have been silenced during the adaptation of $V$. longisporum to crucifers. To test this hypothesis, the goals were set to identify $N L P$ genes of $V$. longisporum that are not expressed and to elucidate the effect of their products on the infection of B. napus. Construction of V. longisporum strains expressing $N L P$ genes that are not expressed in the wild-type strain was the central strategy. Furthermore, the project proposal suggested testing the effect of NLPs on the integrity of plant 
tissue ahead of the fungus, following the hypothesis that NLPs secreted in the hypocotyl are transported with the transpiration stream upwards, reaching the top of the shoot. We speculated that there NLPs would destroy plant tissue, releasing nutrients that would be used at later stages of infection on by the pathogen. In the proposal, we also elaborated on the ability of NLPs to trigger immune responses. The proposal has foreseen that I would test NLPs from $V$. longisporum in Yuelin Zhang's laboratory in the University of British Columbia in Vancouver, Canada, though these experiments were not explicitly set as a scientific goal. After the project started, publications from other laboratories what study NLPs appeared, forcing us to adjust the focus of the work accordingly. Firstly, a publication from T. Nürnberger's laboratory in Tübingen described that a number of peptides derived from NLPs from different organisms acted as MAMPs (Albert et al. 2015). The set of peptides tested in thework included a sequence originating from Fusarium oxysporum, which was nearly identical with a subsequence of cytotoxic NLPs from V. longisporum. The difference occurred at two residues that were shown not be essential for cytotoxicity. Reproducing these results with a purified NLP from our laboratory has not appeared meaningful, but I carried out the experiment in order to learn the techniques and also to benefit from the exposure to a different working environment in Yuelin Zhang's laboratory.

NLP was a hot topic as the project started further key publications appeared. One of these publications revealed the mode of action of cytotoxic NLPs. The team led again by T. Nürnberger showed that NLPs bind to certain sphingolipids anchored in the plasmalemma of dicot plants (Lenarcic et al. 2017) and subsequently causes disintegration of the membrane. The loss of membrane integrity accounts for the cytotoxicity of certain NLPs, which is commonly visualized by infiltrating NLP solutions into leaves of dicot plants. In light of these results and considering that leakage of nutrients in upper shoots of B. napus infected with $V$. longisporum is unlikely to significantly benefit a pathogen that does not colonize this tissue before the onset of senescence, the priority was shifted to tasks that regarded as more promising. Analysis of the expression of $N L P$ genes in $V$. longisporum and construction and testing $V$. longisporum strains overexpressing silent $N L P$ genes remained major tasks of the project.

The enumeration of NLP genes in $V$. longisporum proved difficult due to conflicts among genome databases and contradictions between our sequencing results and database entries. Eventually the discrepancies we resolved and we established that $V$. longisporum harbored 8 NLP 
genes, which were very similar to $N L P$ genes of its likely parent $V$. dahliae (Santhanam et al. 2013, Zhou et al. 2012). These results are described in the first manuscript, which also shows expression analysis of $N L P$ genes in a set of $V$. longisporum strains of different aggressiveness, the construction and testing overexpression mutants, and additional results related to the potential roles of NLPs in the infection of B. napus with $V$. longisporum.

The second project was inspired by the observation that infection of B. napus with $V$. longisporum induced the synthesis of certain phytoalexins. Phytoalexins are plant defense metabolites toxic to fungi and bacteria, supposed to protect plants from pathogens (Ahuja et al. 2012). Numerous fungal pathogens of B. napus were shown to be able to detoxify phytoalexins, which is regarded as a counter-defense activity faciliating colonization of hosts protected by phytoalexins (Pedras and Abdoli 2017). V. longisporum was the only fungal pathogen of B. napus that has not been characterized yet regarding the detoxification of phytoalexins. The circumstances that nearly all work on the detoxification of phytoalexins of B. napus was carried out in the laboratory of Soledade Pedras in Saskatoon, Canada, and that V. longisporum has not been found in Canada before 2015 (Kochgar 2015) might explain this situation. We designed in vitro experiments to determine whether $V$. longisporum can detoxify phytoalexins that are induced by its infection in B. napus, and whether different lineages and strains of $V$. longisporum, including apathogenic isolates, differ in their detoxification activities. Other fungal pathogens and saprophytes have also been tested (see below).

The third project of the thesis was motivated by a critical evaluation of literature about NLPs, which was reviewed in the first project. We realized that the pervasive presence of NLP genes in the genomes of bacteria and fungi of all lifestyles clearly shows that the primary biological function of NLPs is unrelated to plant infection. Even if we would find that NLPs of $V$. longisporum contributed to its aggressiveness, as was shown for its parent $V$. dahliae in tomato (Santhanam et al. 2013), the results would not bring us any closer to the solution of the fundamental questions why NLPs exist and what is their primary function. We found this question fascinating and of prior scientific importance and created a working hypothesis by considering ecological circumstances that are common to bacteria, fungi and fungus-like protists (Oomycetes). These microorganisms obviously share primary metabolism, principles of genetic machinery and structural features that are common to all life on Earth. All these features are indispnesible. NLPs are however dispensable: disruption mutants nut just survive; they usually 
don't show any phenotype. We reasoned that these mutants must have a phenotype but laboratory tests used failed to reveal it, because they missed the conditions under which NLPs unfold their effects. Laboratory examination of gene disruption mutants commonly includes inspection of growth in axenic cultures and for pathogenic microorganisms also infection of hosts. These conditions failed to reveal the fundamental biological function of NLPs. The effects documented in the literature are secondary, as NLPs have been recruted for new functions, which in different phyla happened at different times and affected different activities. We reasoned the the fundamental biological function must be related to a trait that all microbes producing NLPs possess. All microorganisms in the ecosystem interact with other organisms. Because the 3D structure of some NLPs shared similarities with lectins that are toxic to animals (Ottmann et al. 2009), we hypothesized that NLPs are involved in the defense of microbes against animal predators. This hypothesis was in the heart of the third project. We investigated the effect of predation on the transcription of NLP genes in $V$. longisporum, tested the preference of different arthropods for fungal mutants with disrupted NLP genes as compared to strains producing NLPs, and fed nematodes with NLPs produced from cloned genes in E. coli. We also examined the effect of predation on fungal transcriptome. This work led us to the discovery of a widespread defense mechanism in fungi, which became the focus of the third manuscript and involved futher $\mathrm{PhD}$ students and internal as well as external collaborators.

\subsection{Stereotypes in the research on NLPs and phytoalexins may prevent us from asking important questions}

The regulations of GGNB requires that a thesis consisting of manuscripts is equipped with a general discussion section. Although the projects deal with different molecules (proteins and metabolites) and different systems (plant/pathogen and fungus/predator), they possess common features, which can are overarching topics that are discussed in this chapter. The disappointing results of testing traditional working hypothesis of plant pathology regarding the roles of NLPs and phytoalexins in the infection of $V$. napus with $V$. longisporum have raised the first common theme, which is the role of stereotypes in the assignment of function to bioactive molecules in plant pathology. The second common theme, discussed in the next section, is an analogy between 
the concept of ecological metabolites and the ecological functions of proteins mediating nonnutritional interactions among organisms.

In textbooks and even in texts targeting general public (BMBF 2019, Spiegel Online 1991, Hallmann et al. 2007), phytoalexins are described as metabolites that protect plants from microbial infection. The idea that detoxification of phytoalexins helps pathogens to overcome plant defense appears straightforward. The ability of pathogens to detoxify phytoalexins in vitro has been demonstrated in many publications. Conclusive evidence that these activities increased the fitness of pathogens within plants tissue, such as in the case of the detoxification of rishitin by Gibberella pulicaris (Weltring et al. 1998), is however very rare. Even in a textbook example of the detoxification of pisatin by Nectria haematococca (van Etten et al. 1989), which was one of major sources inspiring the phytoalexin hypothesis, the disruption of the gene for pisatin demethylase resulted in only a small decrease of virulence (Ciuffetti and van Etten 1996). As Agrios pointed this out in his textbook (Agrios 2005): "In general, it appears that phytoalexins may play a decisive or an auxiliary role in the defense of some hosts against certain pathogens, but their significance, if any, as factors of disease resistance in most host-pathogen combinations is still unknown" (highlighting by LS). The fact that detoxification of phytoalexins produced by B. napus has been exclusively studied in microorganisms that are able to infect B. napus further weakenes the inference drawn from these studies. An observation that detoxificaton activities are specific for pathogens of B. napus would support the hypothesis that they possess an adaptive value, but non-pathogens have not been included in these studies. Therefore, apart from the investigation of the detoxification of phytoalexins by strains of $V$. longisporum possessing different aggressiveness, we also studied pathogens that do not infect B. napus and saprophytes. This work was carried out by MSc student Cesar Figueroa under my supervision. It turned out that fungi that do not infect oilseed rape were often able to detoxify phytoalexins. These results do not corroborate the hypothesis that pathogens the developed of detoxification activities targeting phytoalexins produced by host plants was instrumental in the adaptation of pathogens to hosts.

The cautionary view of expressed by Agrios in 2005 is still justified today. Detoxification activities of pathogens towards phytoalexins of their hosts are often not as clear-cut as expected (e.g., Denny and van Etten 1983). The results obtained with non-pathogens of oilsed rape raise an intriguing question: Why do fungi possess degradation activities towards phytoalexins that they 
have not encountered in their life histories? We speculate that these activities might originate from enzymes that have different substrates in nature, for instance ecological metabolites produced by microbial competitors. The identification of the enzymes and genes involved in the degradation might be the first step towards clarification of this question.

Investigations of NLPs have been guided by stereotypes, too. The field has been dominated by the idea that NLPs are virulence factors of plant pathogens. The fact that the first NLP was isolated from the pathogen F. oxyporum and that certain NLPs cause spectacular necrosis in dicots may account for this stereotype. Apart from the role of NLPs as virulence factors, research on NLPs in the last years focused on the induction of plant immune response by NLPs acting as MAMPs, and on the mechanism of cytotoxicity of certain NLPs in dicots. The investigation of the MAMP activity of NLPs was very successful, explaining the induction of immune response by NLPs at a molecular level (Albert et al. 2015). The results are important for our understanding of plant defense against microbial infection but they tell us $\mathrm{n}$ nothing about NLPs. The mechanism of cytotoxicity of certain NLPs in dicot plants was investigated an international team lead by the same laboratory, and it was equally successful. The authors identified the molecule binding NLPs to plant membranes and showed why NLPs are toxic to dicots but not to monocots (Lenarcic et al. 2017).

Only a few laboratories were involved in studies of the mode of action of NLPs in dicots and the results led to impressive publications in leading journals. The situation is opposite regarding the research on the role of NLPs as virulence factors in plant infection. Many laboratories working on fungal pathogens tried to obtain support for the hypothesis that NLPs are virulence factors but most published results are negative, inconclusive (including previous results from our laboratory obtained with $V$. longisporum strains with $N L P$ genes silenced with RNAi), or unconvincing because only a single disruption mutant without complementation was tested. Redundancy may explain the failure of first gene disruption experiments but in the meantime all $N L P$ genes have been disrupted in several pathogens, e.g. four genes in M. gresea (Fang et al. 2017), without any detectable effect on virulence. These results are reviewed in the first manuscript. We realized that most work on NLPs of plant pathogens has been carried out with a single strain of a few strains at most and assumed that comparison of strains with different aggressiveness would be helpful. If NLPs are important in pathogenicity, the expression of their genes should be similar among aggressive strains, and it might be different in nonpathogenic strains. We found that expression 
patterns of eight NLP genes of $V$. longisporum varied widely among strains. The expression patterns have not matched the virulence of the strains, contradicted the hypothesis that NLPs of $V$. longisporum are involved in pathogenicity. Overexpression of the $N L P$ genes that are silenced in wild-type strains of $V$. longisporum has not affected the aggressiveness of the pathogen, disproving the hypothesis that silencing these genes was instrumental in the adaptation of $V$. longisporum to crucifers. The hypothesis was motivated by the discovery of MAMP-like activity of NLPs. Our results showed that the products of silenced NLP genes has not prevented or appreciably slowed down plant infection. The situation may be different in other systems but we doubt that the efforts to investigate further pathogens in the same way would be well spent. Investigating the question why pathogens produce NLPs that do not affect their interaction with hosts is more appealing and certainly more relevant.

Critical examination of literature reveals that the number of pathogens in which $N L P$ genes were shown not to affect aggressiveness of their producers might be even higher than conclusions of the publications suggest. The authors often tend to overstate weak positive results, as if they were disappointed that NLPs in their system had not shown the expected effects. For instance, in a recent publication the authors disrupted each of the two NLP genes in Penicillium expansum (Levin et al. 2019) and investigated the aggressiveness of the modified strains on apples. They concluded that "...deletion of Penlp1, but not Penlp2, resulted in reduced virulence on apples manifested by reduced rate of lesion development (disease severity)." Inspection of Fig. 5, which shows the pertinent data, reveals that the conclusion was overstated: the effect was marginal, and only a single mutant was tested without confirmation with an independent mutant or a complementation control. Furthemore, a discrepancy between qPCR and RNAseq results raises further doubts. It seems that the authors were disappointed to find that disruptions of $N L P$ genes caused none or minor effects on infection, though the result was actually more interesting that the exprected effects would be. We believe that negative result of infection assays with NLP mutants stimulate ecological reasoning and advance the field more than finding that cytotoxic proteins enhance aggressiveness of nectrotrophic pathogens. 


\section{3 "Ecological molecules" as a common theme}

The molecules studied this thesis included proteins and secondary metabolites. Fungal proteins were studied in the project on NLPs, plant metabolites in the projects on phytoalexins and bioreactor, and fungal metabolites in the project on fungal defense. Although these projects shared little similarity on the first sight, we noted parallels between certain attributes and of metabolites and proteins studied. We begin by reviewing the role of the exchange of organic molecules among organisms in ecosystems.

Exchange of molecules dominates inter-organismic interactions. Organisms in tropic relationships form food webs. The material that they exchange serves as a source of energy and provides building blocks for anabolic processes. Autotrophic organisms synthesize organic molecules from $\mathrm{CO}_{2}$ and salts using the energy of light (photoautotrophy) or chemical energy released in redox reactions (chemoautotrophy), while heterotrophs degrade organic molecules extracted from the bodies of living or dead autotrophs or other heterotrophs. Most organic molecules exchanged in multitrophic interactions among members of an ecosystem are primary metabolites such as proteins, polysaccharides, lipids and small molecules known as vitamins, which serve as precursors of enzymes. Small metabolites and building blocks of large molecules exchanged in food webs are conserved among all kingdoms of life. The high taxonomic specificity of structures of proteins and nucleic acids does not play any role in food webs because macromolecules are hydrolyzed into monomers by enzymes secreted into the environment or residing in the digestion track of animals. Food webs can only exist if the bodies of all participating organisms are based on the same chemistry. Conservation of primary metabolism is therefore a prerequisite for ecosystem functioning. The exchange of small and large molecules in food webs is well established.

There is a second chemical web spanning all ecosystems, which differ from food webs in several aspects. In contrast to the conservation of primary metabolism and building blocks of biological macromolecules, molecules exchanged in the second chemical web are heterogeneous and diverse. Each molecule is produced by a single species or a small group of species. A second difference is that these molecules are not necessary for growth, development and reproduction of their producers. They play adaptive roles by improving the fitness of their producers under adverse conditions caused by abiotic and biotic stresses such as draught, lack of nutrients, UV 
light, competition, predation, and parasitism. For convenience and due to the lack of a suitable term, the network of non-nutritional interactions among organisms mediated by biomolecules will be called "2 ${ }^{\text {nd }}$ web" here. Molecules exchanged in food webs provide receiving organisms with energy and building blocks for anabolic processes. In the "2 $2^{\text {nd }}$ web", the exchanged molecules convey information and/or modulate physiology or behavior of the receiving organism. The contribution of these molecules to energy uptake and anabolic processes in the target is negligible.

Small molecules participating on the "2 $2^{\text {nd }}$ web" are specialized metabolites (formerly secondary metabolites). Due to their prevailingly ecological roles, these metabolites have also been dubbed "ecological metabolites" (Karlovsky 2008). Ecological roles of macromolecules, which are mainly proteins but as we recently learned can also include RNAs (Hua et al. 2018), are the same as the roles of ecological metabolites: they modulate interactions of organisms with other organisms and with inorganic environment. Because the the thesis focused on interactions among organisms, molecules that protect their producers from abiotic stress or facilitate acquisition of nutrients will not be discussed here.

A common feature of food webs and the "2 ${ }^{\text {nd }}$ web" is that the trafficking of organic molecules is unidirectional. In food webs, nutrients are transferred from hosts, prey and dead bodies to parasites, predators and saprophytes, respectively. In the "2 $2^{\text {nd }}$ web", bioactive molecules move from producers to targets where they unfold their activities.

\subsection{Ecological molecules: proteins or metabolites?}

Specialized metabolites and proteins or peptides involved in non-nutritional interactions among organisms have been studied by different teams using dissimilar methods, and they have been treated separately in ecological considerations. These molecules are functionally equivalent, therefore we suggest embracing them in a common concept. Both kinds of molecules unfold their activity by binding to molecular targets with distinct structural features such as active sites of enzymes, receptors, membrane transporters or ion channels. Specialized metabolites are believed to fulfil ecological functions but these functions could so far be assigned only to a small fraction of known specialized metabolites (Karlovsky 2008). 
In contrast to macromolecules exchanged in food webs, the structures of proteins and RNAs exchanged in the " $2^{\text {nd }}$ web" is essential for their function. Protein and RNA molecules involved in the " $2^{\text {nd }}$ web" have to resist hydrolysis until they fulfill their ecological function, while molecules exchanged in food webs must be hydrolyzed in order to fulfill their ecological role. Similarly as specialized metabolites, macromolecules of the "2nd web" provide negligible energy and biosynthetic precursors to their target organisms.

Proteins and peptides control interactions among organisms by acting as effectors, toxins or physiological modulators. They cause the same sort of impacts as ecological metabolites. The term "ecological proteins" therefore appears suitable but because it is not established, it will be use it in quotation marks. "Ecological proteins" may kill fungal predators or deter them from feeding (Künzler 2015) or immobilize and kill the prey of carnivore animals (Chen et al. 2018), which are functions similar to the function of bis-naphthopyrones shown in our work to act as ubiquitous antifeedant (third manuscript). We hypothesized that NLPs act as "ecological proteins", protecting their producers from predation. The hypothesis was not supported but NLPs are still likely to belong to "ecological proteins", the biological function of which remains enigmatic. Effectors of plant pathogens that suppress plant defense (Toruno et al. 2016) and plant defense proteins targeting herbivores (Broadway et al. 1986, Peumanns and Van Damme 1995) fulfill the definition of "ecological proteins", too. Most research on NLPs so far focused on their potential roles in the infection of host plants by pathogens. Although the primary function of NLPs as "ecological proteins" cannot be related to plant infection, some pathogens seem to have recruited NLPs as factors supporting infection (Santhenam at al. 2013). The fact that NLPs serves as signals triggering plant defense responses (Albert et al. 2015) does not support their assignment to "ecological proteins" for the same reason as bacterial flagellin or fungal chitin do not belong to ecological molecules though they trigger defense responses in plants.

An interesting question is the position of enzymatic activities degrading phytoalexins in this system. Phytoalexins are clearly part of the "2 $2^{\text {nd }}$ web". Enzymes of pathogens that degrade phytoalexins are dispensable. They enhance the fitness of their producers under adverse conditions, which are landmarks of "ecological proteins". However, these enzymes are not exchanged among the interacting organisms, which would exclude them from the "2 $2^{\text {nd }}$ web". This illustrates the limits of the suggested classification system. Some enzymes degrading phytoalexins might be secreted into plant tissue, which means that they traffic between their 
producer and the target organisms. Thus these enzymes fulfill our definition of "ecological proteins". It appears artificial to assign extracellular but not intracellular degrading enzymes to the "2 ${ }^{\text {nd }}$ web". We therefore feel that the definition should be refined to include molecules that do not travel between interacting organisms but act upon molecules that have been exchanged.

\section{References}

Agrios, G. N. 2005. Plant Pathology. 5th ed., Academic Press: Amsterdam, Boston.

Ahuja, I., Kissen, R., and Bones, A. M. 2012. Phytoalexins in defense against pathogens. Trends Plant Sci. 17:73-90.

Albert, I., Böhm, H., Albert, M., Feiler, C. E., Imkampe, J., Wallmeroth, N., et al. 2015. An RLP23-SOBIR1-BAK1 complex mediates NLP-triggered immunity. Nat Plants. 1:15140.

BMBF. Pflanzenforschung. Available at: https://www.pflanzenforschung.de/de/themen/lexikon/antimikrobiell-1141 [Accessed August 29, 2019].

Broadway, R. M., Duffey, S. S., Pearce, G., and Ryan, C. A. 1986. Plant proteinase inhibitors: a defense against herbivorous insects? Entomologia Experimentalis et Applicata. 41:33-38.

Chen, N., Xu, S., Zhang, Y., and Wang, F. 2018. Animal protein toxins: origins and therapeutic applications. Biophys Rep. 4:233-242.

Ciuffetti, L. M. (Cornell U., and VanEtten, H. D. 1996. Virulence of a pisatin demethylase-deficient Nectria haematococca MPVI isolate is increased by transformation with a pisatin demethylase gene. Molecular plantmicrobe interactions : MPMI (USA). Available at: http://agris.fao.org/agris-search/search.do?recordID=US9708839 [Accessed August 31, 2019].

Denny, T. P., and Vanetten, H. D. 1983. Tolerance of Nectria haematococca MP VI to the phytoalexin pisatin in the absence of detoxification. Microbiology,. 129:2893-2901.

Fang, Y.-L., Peng, Y.-L., and Fan, J. 2017. The Nep1-like protein family of Magnaporthe oryzae is dispensable for the infection of rice plants. Sci Rep. 7:1-10.

Hallmann, J., Quadt-Hallmann, A., and von Tiedemann, A. 2007. Phytomedizin Grundwissen Bachelor. Ulmer Publisher.

Hua, C., Zhao, J.-H., and Guo, H.-S. 2018. Trans-Kingdom RNA Silencing in Plant-Fungal Pathogen Interactions. Molecular Plant. 11:235-244.

Karlovsky, P. 2008. Secondary Metabolites in Soil Ecology. In Secondary Metabolites in Soil Ecology, Soil Biology, ed. Petr Karlovsky. Berlin, Heidelberg: Springer Berlin Heidelberg, p. 1-19.

Kochhar, K. 2015. Latest Pest Reports - International Plant Protection Convention. Available at: https://www.ippc.int/en/countries/canada/pestreports/2015/11/january-2015-detection-of-verticillium-wilt-of-canolaverticillium-longisporum-in-manitoba/ [Accessed August 29, 2019].

Künzler, M. 2015. Hitting the sweet spot-glycans as targets of fungal defense effector proteins. Molecules. 20:81448167.

Lenarčič, T., Albert, I., Böhm, H., Hodnik, V., Pirc, K., Zavec, A. B., et al. 2017. Eudicot plant-specific sphingolipids determine host selectivity of microbial NLP cytolysins. Science. 358:1431-1434.

Levin, E., Raphael, G., Ma, J., Ballester, A.-R., Feygenberg, O., Norelli, J., et al. 2019. Identification and functional analysis of NLP-ecoding genes from the postharvest pathogen Penicillium expansum. Microorganisms. 7:175.

Oome, S., Raaymakers, T. M., Cabral, A., Samwel, S., Böhm, H., Albert, I., et al. 2014. Nep1-like proteins from three kingdoms of life act as a microbe-associated molecular pattern in Arabidopsis. PNAS. 111:16955-16960. 
Ottmann, C., Luberacki, B., Küfner, I., Koch, W., Brunner, F., Weyand, M., et al. 2009. A common toxin fold mediates microbial attack and plant defense. PNAS. 106:10359-10364.

Pedras, M. S. C., and Abdoli, A. 2017. Pathogen inactivation of cruciferous phytoalexins: detoxification reactions, enzymes and inhibitors. RSC Adv. 7:23633-23646.

Peumans, W. J., and Damme, E. V. 1995. Lectins as plant defense proteins. Plant Physiology. 109:347-352.

Roongsawang, N., Washio, K., and Morikawa, M. 2011. Diversity of nonribosomal peptide synthetases involved in the biosynthesis of lipopeptide biosurfactants. International Journal of Molecular Sciences. 12:141-172.

Santhanam, P., van Esse, H. P., Albert, I., Faino, L., Nürnberger, T., and Thomma, B. P. H. J. 2013. Evidence for functional diversification within a fungal NEP1-like protein family. Mol. Plant Microbe Interact. 26:278-286.

Spiegel Online. 1991. Pflanzenschutz: Eleganter Ersatz. Available at: https://www.spiegel.de/spiegel/print/d13490358.html [Accessed August 29, 2019].

Toruño, T. Y., Stergiopoulos, I., and Coaker, G. 2016. Plant-pathogen effectors: cellular probes interfering with plant defenses in spatial and temporal manners. Annual Review of Phytopathology. 54:419-441.

VanEtten, H. D., Matthews, D. E., and Matthews, P. S. 1989. Phytoalexin Detoxification: importance for pathogenicity and practical implications. Annual Review of Phytopathology. 27:143-164.

Weltring, K.-M., Loser, K., and Weimer, J. 1998. Genetic instability of rishitin metabolism and tolerance and virulence on potato tubers of a strain of gibberella pulicaris. Journal of Phytopathology. 146:393-398. 


\section{Article I: Elucidation of biological roles of NLPs of Verticillium longisporum in interaction with Brassica napus and fungivorous invertebrates}

Ling $\mathrm{Su}^{1}$, Yang Yuan $^{1}$, Markus Arndt ${ }^{1}$, Haidi Yin $^{1}$, Malte Beinhoff ${ }^{1}$, Susanna A. BrausStromeyer $^{2}$, Gehard H. Braus ${ }^{2}$, Yuelin Zhang ${ }^{3}$, Petr Karlovsky ${ }^{1 *}$

${ }^{1}$ University of Goettingen, Molecular Phytopathology and Mycotoxin Research, 37077 Göttingen, Germany

${ }^{2}$ University of Goettingen, Institute of Microbiology and Genetics, 37077 Göttingen, Germany

${ }^{3}$ University of British Columbia, Department of Botany, V6T 1Z4, Vancouver, BC Canada

*Corresponding authors: PK

Emails: pkarlov@gwdg.de

\section{Author contribution:}

Ling Su designed and carried out all experiments on VINLPs project except for the expression and purification of VINLP1. She guided MSc student Yang Yuan, BSc student Markus Arndt and student assistant Zaheer Abbas in conducting fungal transformation of VINLPs overexpression constructs and related plant test, food preference and fitness assay, and some RNA extraction, respectively. Partial infiltration experiments were done by Haidi Yin. Data were processed by Ms. Su alone, except for $V$. longisporum DNA quantification in plants, which was done by Ms. Yuan; food choice and fitness assay data were processed by Mr. Arndt. Ling Su wrote the entire manuscript. 


\section{Abstract}

The ascomycete Verticillium longisporum is an allodiploid hybrid adapted to infecting crucifers. Necrosis and Ethylene Inducing Peptide 1(Nep 1)-Like proteins (NLPs) are proteins sharing similarity with Nep 1 of Fusarium oxysporum. Some NLPs are cytotoxic in dicot plants and some induce plant defense responses. We found that the genome of $V$. longisporum harbors $8 N L P$ genes which are similar to $N L P$ genes of $V$. dahliae. The purified product of VlNLPl was cytotoxic in Arabidopsis, Brassica napus, and Nicotiana tabacum and triggered immune response in Arabidopsis. The expression pattern of NLP genes varied among the lineages of $V$. longisporum and among strains within a lineage. No correlation between the expression patterns of NLPs and strain aggressiveness was found. For example, VINLP3 was the only VINLP gene, the expression of which was not detectable in the virulent strain VL43 nor in the avirulent strains VL1198 and VL1199. VINLP3 was expressed to a high level in the avirulent strain VL32 and at low levels in the avirulent strain VL787 and the virulent strain VL1194. All VINLPs were expressed in VL32, indicating that NLPs in V. longisporum are not virulence factors. To test whether VINLP3 triggers plant responses that suppress disease symptoms, VINLP3 and VINLP2, which are very similar, were expressed in genetically engineered strain VL43. Infection of B. napus with two overexpression strains was indistinguishable from the infection with the wild type strain, showing that silencing VINLP3 was not necessary for the adaptation of $V$. longisporum to B. napus. Interestingly, overexpression of both VINLP2 and VINLP3 enhanced the transcription of $V I N L P 1$, indicating that $V l N L P$ genes are controlled by a shared regulatory circuit. In a search for an alternative biological function of NLPs, the similarity of NLPs to certain lectins inspired a hypothesis that NLPs protect microorganisms from animal predators. Predation by the nematode Aphelenchoides saprophilus or springtail Folsomia candida has not affected the expression of eight NLP genes in $V$. verticillium nor three NLP genes in Fusarium graminearum. The springtail $F$. candida has not distinguished between the wild type strain of Zymoseptoria tritici and a mutant with the only NLP gene disrupted in food choice experiments. Feeding $F$. candida on the $Z$. tritici strain with the disrupted NLP gene has not enhanced the fitness of the springtail as compared to feeding on the wild type strains accumulating NLP. In summary, these experiments show that NLPs of $V$. longisporum play none or marginal role in the infection of B.napus, and also indicate that NLPs of V. longisporum, F. graminearum and Z. tritici are not involved in defense against animal predation. 


\subsection{Introduction}

\subsubsection{The fungal genus Verticillium}

The ascomycete genus Verticillium currently contains ten soil-borne species (Inderbitzin et al. 2011a), including V. longisporum, V. alfalfae, V. nonalfalfae, V. albo-atrum, V. nubilum, $V$ dahliae, V. isaacii, V. tricorpus, V. zaregamsianum, and V. klebahnii (Inderbitzin et al. 2011a; Inderbitzin and Subbarao 2014). Among them, V. dahliae, V. longisporum and V. albo-atrum are described as the most economically important species, especially $V$. dahliae causing vascular wilting disease in more than 200 plant host species (Inderbitzin et al. 2011a; Pegg and Brady 2002). The estimated economic loss in one year caused by Verticillium disease can reach $€ 3$ billion over the world (Depotter et al. 2016). Germany is one of the main regions for the production of oilseed rape (Brassica napus) in Europe following France (Eurostat 2019). Therefore, research on $V$. longisporum, as one of the major oilseed rape pathogens, is becoming increasingly important. However, the yield loss in oilseed rape caused by $V$. longisporum under field condition varies estimated to range from 10 to $50 \%$ (Dunker et al. 2008).

$V$. longisporum was first isolated from horseradish and described as V. dahliae var. longisporum (Stark 1961). Although the authors found that conidia of $V$. longisporum were twice as long as $V$. dahliae and the oblong nuclei of $V$. longisporum differed from the spherical nucleus of $V$. dahliae, $V$. longisporum was not regarded as a distinct species for a long time. More than three decades later, Karapapa et. al. elevated V. longisporum to an independent species but until recently, V. dahliae and V. longisporum could not be easily distinguished (Karapapa et al. 1997). This re-classification is very critical, because these two fungi do not share plant hosts. To be specific, $V$. longisporum has only been observed to infect brassicaceous hosts in the field, while $V$. dahliae rarely infects crucifers but has a wide host range of non-cruciferous species (Inderbitzin and Subbarao 2014). Besides, V. longisporum contains about 1.7-1.8 times more nuclear DNA than $V$. dahliae, therefore it has been referred to as 'near-diploid' or amphidiploid (Karapapa et al. 1997; Collins et al. 2003; Steventon et al. 2002).

$V$. longisporum and $V$. dahliae are soil-borne plant pathogens that have similar life cycles (Fig. 1). Both pathogens are capable of producing black melanized microsclerotia and surviving in this dormant form for a long time until germination that is induced by root exudates of host and even 
non-host plants, followed by hyphal growth directed towards the roots (Berlanger, I. and Powelson, M.L. 2000; Leino 2006). When the hyphae reach root hairs, they grow towards the root surface and penetrate it. Natural root wounds are another way how the pathogens enter the host plants (Karapapa et al. 1997). Inside the root cortex, hyphae grow inter- and intracellularly towards the central cylinder and eventually colonize xylem vessels (Eynck et al. 2007) and clumped at the end of a tracheid. These conidia are able to germinate and penetrate into adjoining vessels (Garber, R. H. and Houston, B. R. 1966). Due to the restricted colonization of $V$. longisporum in individual xylem vessels, the transpiration stream is not disturbed by occluding xylem vessels (Kamble et al. 2013). In senescing plants V. longisporum breaks out of xylem, microsclerotia are formed in the tissue. With plant debris microsclerotia are deposited on the soil surface or plugged into the soil where they allegedly can survive up to 15 years (Fradin and Thomma 2006). A recent study has found that $V$. longisporum is capable to transmit by the seed of oilseed rapes under greenhouse condition and by the seed of spring oilseed rape in the field (Zheng et al. 2019a). It is important to note that the disease development and symptoms in oilseed rape after artificial infection with $V$. longisporum by root dipping differs from the disease development and symptoms observed under field conditions. The typical field symptom, which is dark unilateral striping on the stem, is missing in artificially inoculated plants, while the symptoms observed in the greenhouse, which are chlorosis and stunting at early growth stages, are missing in infected plants in the field (Eynck et al. 2007; Eynck et al. 2009; ZEISE and Tiedemann 2002). 


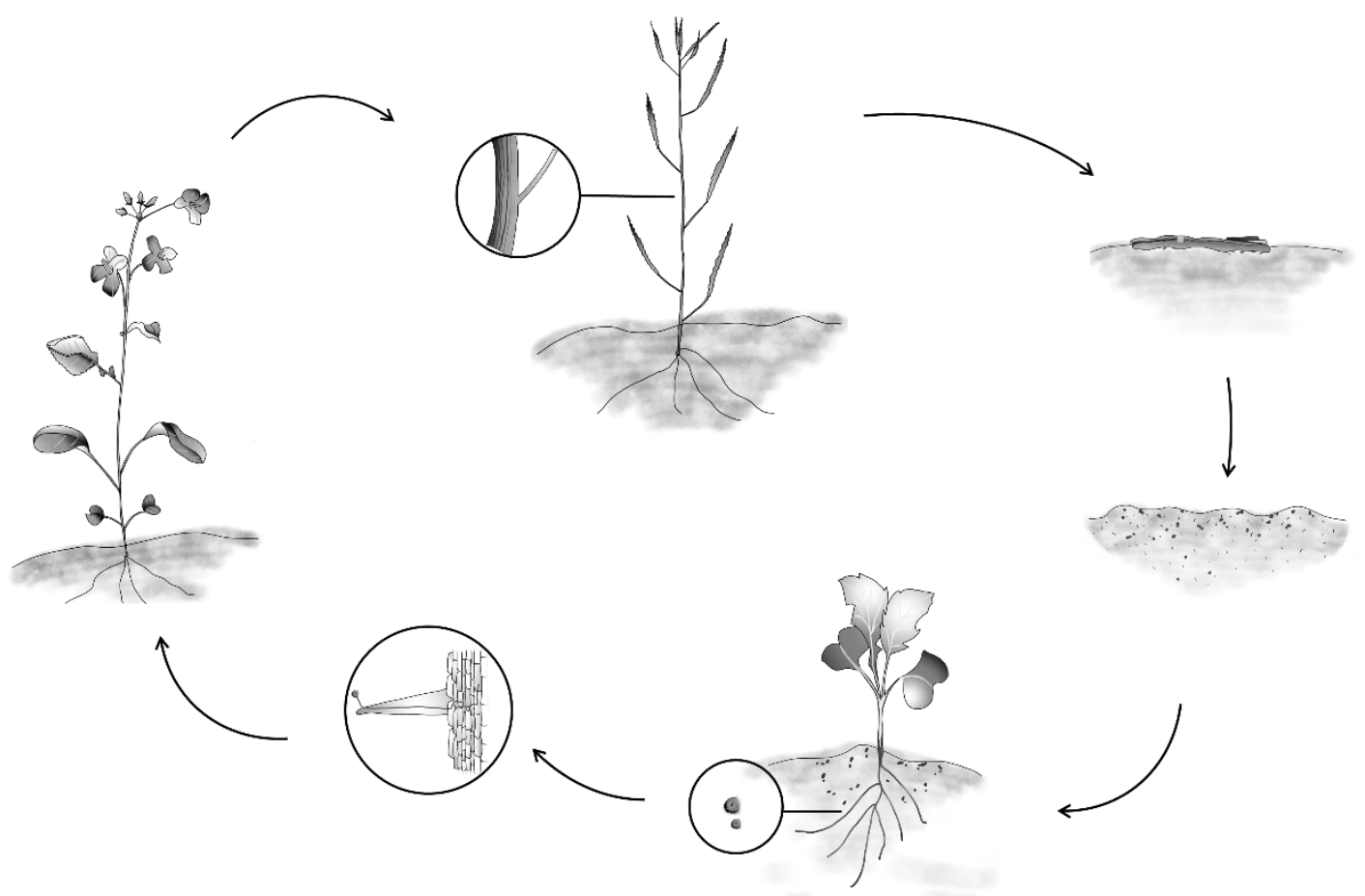

Fig. 1 Disease cycle of Verticillium longisporum on Brassica napus.

1. Microsclerotia reside in soil; 2. Root exudates trigger microsclerotia germination and hyphae grow toward roots; 3. V. longisporum penetrates the epidermal cells of lateral roots or enter through root wounds; 4. Before ripening, crops remain asymptomatic; 5. During ripening, dark unilateral striping are observed on plant stem; 6 . Microsclerotia are formed and released with plant debris in to soil. The figure was modified based on Depotter et al. (2016).

As indicated by its genome size and molecular marker studies, V. longisporum is a merodiploid (amphihaploid) hybrid deriving from two haploid species (Collins et al. 2005; Clewes et al. 2008). Comparison of sequences of 7 single-copy genes in a large set of $V$. longisporum isolates established that hybridization events leading to $V$. longisporum occurred at least three times and involved four parents comprising two lineages of $V$. dahliae and two yet undescribed Verticillium species (Inderbitzin et al. 2011b).

Hybridization of A1 with D1, D2 and D3 formed three $V$. longisporum lineages A1/D1, A1/D2 and A1/D3, respectively (Fig. 2). Among the parents, A1 and D1 is unknown species while D2 and D3 are considered to be lineages of $V$. dahliae (Inderbitzin et al. 2013) (Fig. 2). 


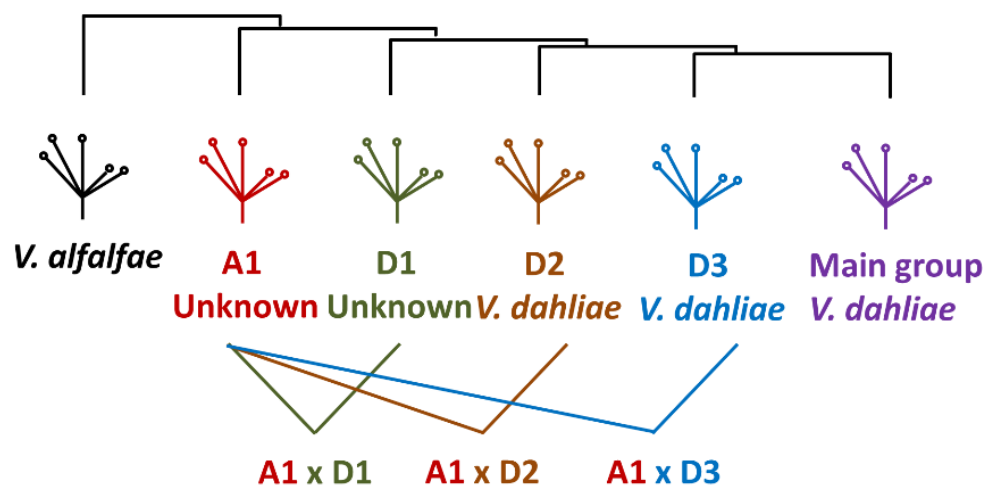

Fig. 2 The three lineages of Verticillium longisporum A. Phylogenic analysis of putative parents of $V$. longisporum. B. The three lineages A1/D1, A1/D2 and A1/D3 are hybridized by the combination of two unknown Verticillium species A1, D1 and two $V$. dahliae species D2 and D3, respectively. The figure was modified based on Depotter et al. (2016).

Because the lineages of $V$. longisporum cannot be differentiated by morphological characters, the molecular technique is used for lineages identification. PCR primers designed by Inderbitzin et al. (2013) for multiple PCR markers are commonly used to distinguishing the lineages of $V$. longisporum from each other and from V. dahliae.

Distinct differences in virulence and pathogenicity among the lineages of $V$. longisporum lineages and between $V$. longisporum and $V$. dahliae were demonstrated (Novakazi et al. 2015). All three lineages of $V$. longisporum are more virulent on crucifers than $V$. dahliae; differences among lineages in aggressiveness on crucifers have been observed, but the variation among isolates within lineages was large. The lineage A1/D1 is the most virulent group on all Brassicaceae plants. Lineages A1/D1 and A1/D3 show host specificity for oilseed rape and cauliflower, while A1/D2 is specific to horseradish. Interestingly, the most aggressive strain found so far on oilseed rape (VL1194) belongs to the lineage A1/D3. The overwhelming majority of strains in this lineage are less virulent on all tested host plants as compared to strains of the A1/D1 lineage. It is generally assumed that $V$. dahliae is more aggressive than $V$. longisporum on all host plants except Brassicaceae. Unexpectedly, one $V$. longisporum strain was demonstrated to be as virulent as $V$. dahliae on tomato, lettuce, eggplant and even watermelon, which indicates that the host range of $V$. longisporum is much wider than only Brassicaceae under conditions of artificial inoculation (Novakazi et al. 2015). 


\subsubsection{Necrosis and ethylene-inducing peptide 1 (Nep 1)-like proteins (NLPs)}

Necrosis and ethylene-inducing peptide 1 (Nep 1)-like proteins (NLPs) are distributed among a wide range of prokaryotic and eukaryotic microorganisms with diverse lifestyles, including the kingdoms of fungi, protists (Oomycetes) and eubacteria (Pemberton and Salmond 2004; Gijzen and Nürnberger 2006). The first characterized NLP, designated Nep 1, was $24 \mathrm{kDa}$ protein isolated from culture filtrates of the vascular wilt fungus Fusarium oxysporum. The infiltration of Nep 1 into various dicotyledonous plants, but not monocotyledon, caused necrosis on the leaves and induced ethylene production (Bailey 1996). The necrosis triggered by NLPs is different from the programmed cell death associated with plant immune response (Qutob et al. 2006). Proteins of the NLP family contain a conserved NPP1 domain containing a strongly conserved sevenamino-acid motif, GHRHDWE (Fellbrich et al. 2002). Based on the NPP1 domain, the Pfam domain PF05630 was defined (Fellbrich et al. 2002). An overwhelming majority of NLPs contain an N-terminal signal peptide (SP), indicating that they are secreted, and many NLPs have indeed been isolated from culture media. When NLP genes are expressed in plants, their product trigger necrosis only when the NLPs are secreted into the apoplast and thus can interact with the outer surface of plasmalemma (Qutob et al. 2003; Qutob et al. 2006). The cytotoxic activity of NLPs, the plant immune defense triggered by NLPs and the effect of NLPs on the aggressiveness and virulence of pathogens are the three most studied topics in research on NLPs.

Three NLP subfamilies are distinguished based on the presence of conserved cysteine residues. In 2006, type 1 NLP containing two conserved cysteines and type 2 NLP with four cysteines were defined by Nürnberger group (Gijzen and Nürnberger 2006). With the increasing number characterized NLP genes, the third type of NLP was defined (Oome and van den Ackerveken 2014), which differs from the types 1 and 2 to a larger degree than they differ from each other. While all type 1 and type 2 NLPs contain the entire NPP1 domain, type 3 NLPs share only a central sequence (around 50 amino acids) including the conserved heptapeptide motif. Moreover, the six cysteine residues of type 3 NLPs are not located near the "GHRHDWE" motif, hence they do not match the type 1 and type 2 disulfide bridge pattern. NLP ${ }_{P y a}$ found in the oomycete Phythium aphanidermatum (Oome and van den Ackerveken 2014; Ottmann et al. 2009), NLP Pcc identified in the soft-rot bacterium Erwinia (Pectobacterium) carotovora subsp. carotovora (Mattinen et al. 2004) and NLP from the fungus Aspergillus fumigatus (Afu5g02100) (Oome and 
van den Ackerveken 2014) were selected to show the architecture of the three NLP types in Fig. 3.

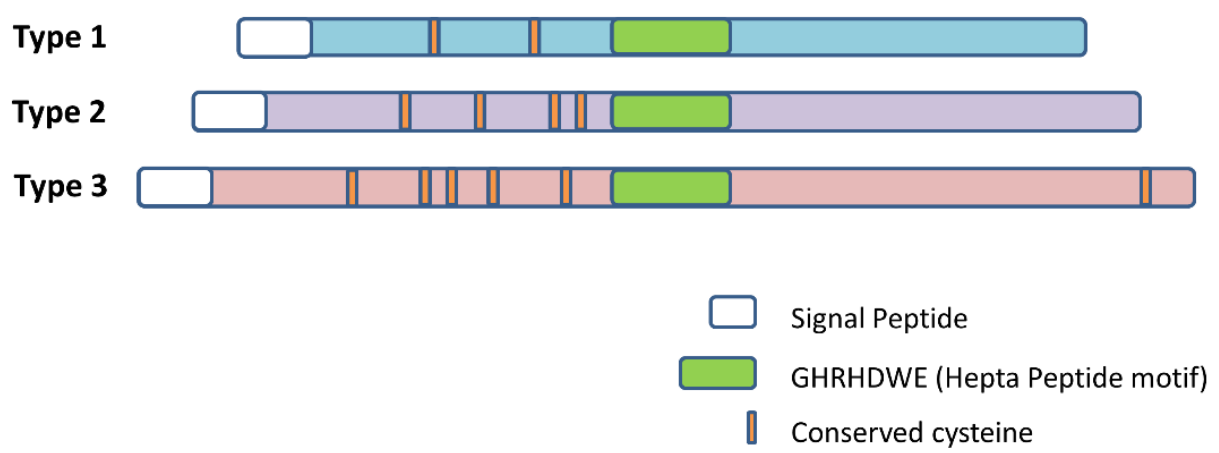

Fig. 3 Schematic representation of three types of NLPs. Phythium aphanidermatum, Erwinia (Pectobacterium) carotovora subsp. carotovora and Aspergillus fumigatus (Afu5g02100) were used as representatives for type 1, type 2 and type 3 NLPs. Type 1 NLPs contain two conserved cysteines and type 2 NLPs have four cysteines, while six cysteine residues of type 3 NLPs are not located near the "GHRHDWE" motif, hence they do not match the type 1 and type 2 disulfide bridge pattern. The figure was modified based on Oome and van den Ackerveken 2014).

The distribution of 533 NLP genes in more than 150 species showed that type 1 NLPs are present in fungi, oomycetes and bacteria; type 2 NLPs are found in fungi and bacteria, and type 3 NLPs only occur in fungi (Fig. 4). All three types of NLPs in a single species are only found in Ascomycetes, such as in the species Verticillium studied in this work. It indicates that NLPs might have originated in the phylum Ascomycota. Studying NLPs of Ascomycetes might help to clarify the evolutionary history of NLPs. Horizontal gene transfer of NLPs from Ascomycetes to other organisms have been suggested (Oome and van den Ackerveken 2014), but origin in a common ancestor followed by a selective loss of certain NLP types in some evolutionary lineages is also conceivable. 

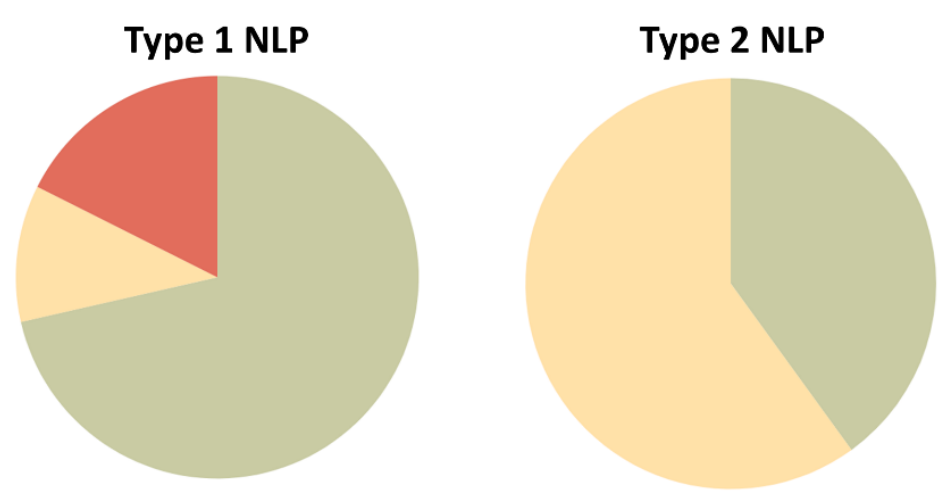

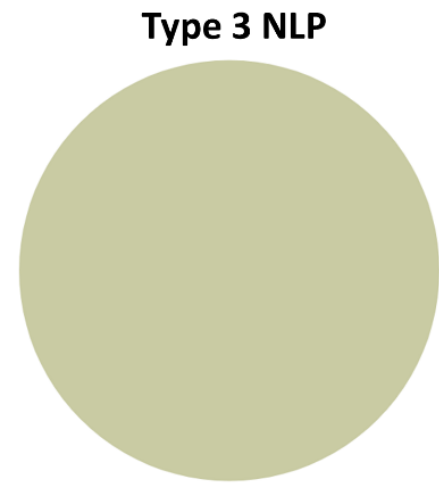

\section{Fungi Bacteria Oomycete}

Fig. 4 Distribution of three types of NLPs among three kingdoms. In all analyzed species containing NLPs, 91 species are type 1; 100 species are type 2 and 31 species are type 3 . Besides, fungi contain most species with type 1 and all species with type 3 NLPs. The figure was modified based on Oome and van den Ackerveken (2014).

Type 1 NLPs are classified based on a single disulfide bridge. This disulfide bridge is required for necrosis (Ottmann et al. 2009). Type 1 NLPs have been identified in fungal species that do not infect plants such as the cellar mold Zasmidium cellare (Crous et al. 2009), saprophytic species of the genera Neurospora and Aspergillus and mycoparasitic Trichoderma spp. (Schmoll et al. 2016). Pathogens of monocot plants have NLPs as well, such as the genera Magnaporthe, Setosphaeria, Phrenophora and Cochliobolus, though NLPs do not cause necroses in monocots (Bailey 1996).

Type 2 and type 1 NLPs share an overall structure. They differ by a second disulfide bridge in NLPs type 2, but which is lacking in type 1. The second disulfide bridge is not necessary for necrosis formation. In addition, type 2 NLPs have some conserved motifs that differ from type 1. For example, type 2 has a potential motif for binding calcium (Rigden and Galperin 2004). Most of type 2 NLPs are also found in the Ascomycota. In addition to the plant pathogens, entomopathogenic microorganisms and animal pathogens harbour type 2 NLPs as well (Vega et al. 2009; Santos et al. 2011).

Type 3 NLPs are special in not sharing homology with the other two types, except for the motif "GHRHDWE" and its surrounding amino acid residues, which form an acidic cation-binding 
sequence. Type 3 NLPs have only been identified in the Ascomycetes which were suggested as the origin of the entire NLPs family. Due to large differences among type 3 NLPs, it is difficult to build a representative three-dimension model. Although not many type 3 NLPs have been identified compared with the other two types, species not pathogenic to plants harbour this NLP type as well, such as the bat pathogen Geomyces destructans and the nematode pathogen Arthrobotrys oligospora (Albert et al. 2015; Oome and van den Ackerveken 2014).

In summary, the types of NLPs are defined by the presence of disulfide bridges and by sequence homology. All three types are found in species that do not infect plants, including saprophytes, insect pathogens and animal pathogens. Therefore, the primary biological function of NLPs is unlikely related to plant pathogenicity.

\subsubsection{Cytotoxicity of NLPs and their activity as MAMPs}

Since the first NLP from $F$. oxysporum was characterized, the cytotoxicity and necrosis-inducing activity of NLPs from a broad range of microbes have been demonstrated on dicotyledonous plants, but not on monocots (Santhanam et al. 2013; Mattinen et al. 2004; Feng et al. 2014). A signal peptide (SP) is necessary for the necrosis formation by NLP transiently expressed in plant cells, which indicated that the functional target of NLPs is extracellular of the outer surface of the plasma membrane of eudicot plants (Qutob et al. 2006). The glycosylinositol phosphorylceramide (GIPC) sphingolipids localized on the outer side of plasmalemma were identified as the molecules binding NLPs in a recent study (Lenarčič et al. 2017). The reason why NLPs does not cause necrosis in monocots was also explained. As shown in Fig. 5, the length of GIPC sugar head of monocots with three hexose units (called series B GIPC) is longer than the sugar head of dicots with two terminal hexoses (called series A GIPC). It means that the series A GIPCs of dicots is closer to the cell membrane surface than series B. The larger distance of NLPs bound to GIPCs in monocots from the membrane would prevent interaction with lipid targets in the membrane. 

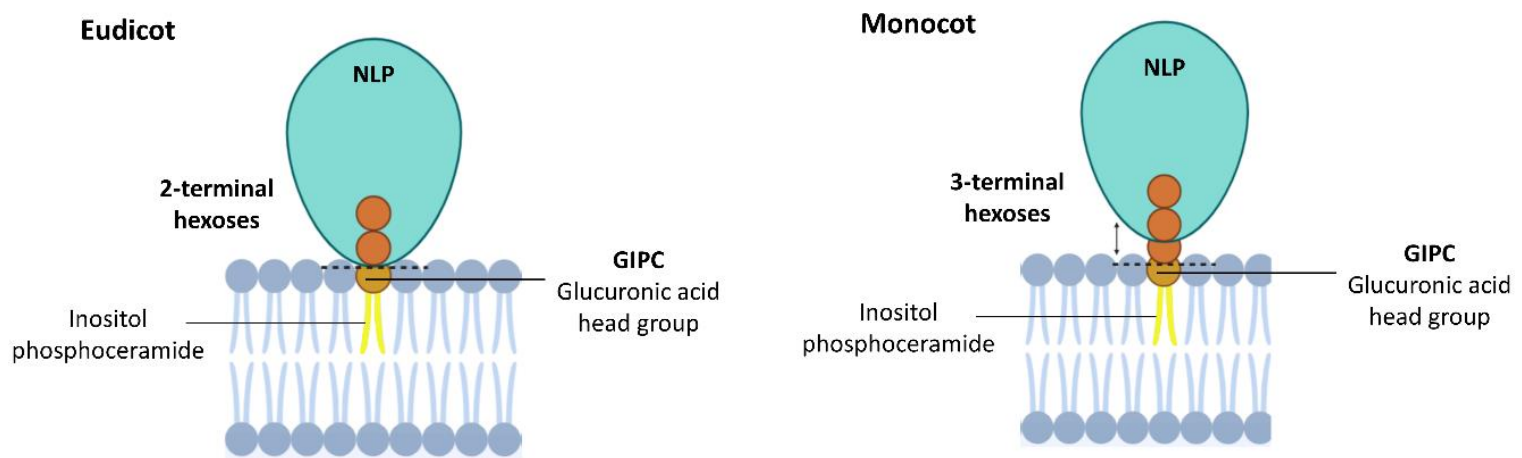

Fig. 5 Schematic figure of NLP cytotoxin specificity. The sphingolipid GIPCs of dicots have one less hexose than monocots, resulting in closer to the cell membrane surface and easier contact and insert into lipid bilayer. The figure was modified based on van den Ackerveken (2017).

In addition to SP, the disulfide bridge A formed by the first pairs of conserved cysteine is essential for necrosis, but the bridge B which only exist in type 2 NLP is not required (Oome and van den Ackerveken 2014). The integrity of proteins is also necessary (Veit et al. 2001).

Plasma membrane leakage caused by NLPs was elucidated by in vitro studies of permeabilization of membrane vesicles derived from dicot lipids (Ottmann et al. 2009). Permeabilization of the vesicles showed that NLPs were cytolytic toxins. Several amino acid residues located in the conserved region of the NPP1 domain and its heptapeptide motif (GHRHDWE) were essential for the permeabilization of membrane vesicles. Since the permeabilizing ability of NLPs inevitably leads to damage of plant cells, NLPs could act as damage-associated molecular patterns (DAMP), which induce plant immune response differently from pathogen-associated molecular patterns (PAMP).

Most of the cytotoxic NLPs belong to type 1, but necrosis-inducing abilities were also identified in type 2 and type 3 NLPs, such as type 2 NLP from Pectobacterium (Erwinia) carotovorum subsp. carotovorum (Mattinen et al. 2004) and type 3 NLP from Penicillium expansum (Levin et al. 2019). Most of NLPs are not cytotoxic. For example, seven out of nine VdNLPs from $V$. dahliae isolate V592 (Zhou et al. 2012), eleven out of nineteen PsNLPs in Phytophthora sojae (Kanneganti et al. 2006), two out of three PiNLPS in Phytophthora infestans (Kleemann et al. 2012) and twelve HaNLPs tested in Hyaloperonospora arabidopsidis are not able to cause 
necrosis in leaves of dicot plants (Chen et al. 2018). Therefore, all the evidence indicates that the initial role of NLPs is not related to the damage of plant cells.

Innate immune systems protect plants from microbial infection (Macho and Zipfel 2014). Plant immune responses start with the recognition of pathogen-associated molecular patterns (PAMP) or microbe-associated molecular patterns (MAPM) by pattern recognition receptors (PRRs) in a process called PAMP-triggered immunity (PTI) or MAPM-triggered immunity (MTI) (Böhm et al. 2014a; Gust and Felix 2014). During the co-evolution of plants and their pathogens, some microbes developed effectors in order to suppress PTI. Host plants, in turn, developed mechanisms triggering immune responses based on the recognition of effectors, called effectortriggered immunity (ETI) (Dou and Zhou 2012). Plant immune responses are subsequently activated, such as the production of reactive oxygen species, ethylene biosynthesis, PR protein expression, callose apposition and activation of mitogen-activated protein (MAP) kinase cascades (Thomma et al. 2011; Tsuda and Katagiri 2010; Nitta et al. 2014). In addition, the damage of plant cells inflicted by toxicity is also able to trigger plant immunity.

A certain conserved region of MAMPs is sufficient to trigger the basal immune response in plants. For instance, a 22 amino acids fragment of flagellin (flg22) can be recognized by proteins designated LRR PRRs and cause MTI in host plants (Felix et al. 1999). LRR PRRs include two groups, LRR-receptor kinase PRRs (LRR-RKs) and LRR-receptor protein PRRs (LRR-RPs), which mediate pattern binding and initiate intracellular signal transduction (Böhm et al. 2014a; Gust and Felix 2014). In addition, receptors usually work in complexes with co-receptors during signal transduction (Gust and Felix 2014).

Various necrosis and ethylene-inducing peptide 1 (Nep1)-like proteins (NLPs) act as MAMPs, triggering plant immune responses. As far as we know, NLP family is the first proteinaceous MAMP spread among three kingdoms of microorganisms. A pattern of 20 amino acids residues of NLP protein (nlp20) is sufficient to active plant immunity in different Brassicaceae species, including Arabidopsis. Unlike cytotoxicity, only type 1 NLPs was reported to be able to trigger plant immune responses. Nlp 20 is able to bind to RLP23 (LRR-RP), following a tripartite complex formation in a ligand-independent manner with SOBIR1 (Suppressor of Brassinosteroid insensitive 1 (BRI1)-associated kinase (BAK1)-interacting receptor kinase 1), and in a liganddependent fashion with BAK 1. In short, NLP-triggered immunity is mediated by a receptor 
complex RLP23-SOBIR1-BAK1 (Albert et al. 2015). Pretreatment of plants with NLPs increased plant resistance to other pathogens (Albert et al. 2015).

\subsubsection{Fungal defense against predators}

The biomass of soil fungi is a nutrient-rich food for soil invertebrates, such as mites, collembolans and nematodes. In the long co-evolutionary process, fungi developed chemical defense mediated by proteins or secondary metabolites in order to protect themselves from predation. Fungal toxins have been investigated extensively for their potential role as defense compounds acting against fungal predators (Janzen 1977). Our work in Article III revealed that a nontoxic pigment, bis-naphthopyrone aurofusarin, protects Fusarium fungi from predators, including springtails, woodlice and insects.

All members of the fungal genus Fusarium are plant pathogens, and all colonize in plant residues on the soil surface. They are famous for their capacity of mycotoxin production. Fusarium mycotoxins deoxynivalenol (DON), zearalenone (ZEA) and fumonisin B1 (FB1) endanger the health of consumers and life stock. Therefore, mycotoxin production in this fungal genus has been studied extensively. The role of mycotoxins of Fusarium in defense against predators has however not been supported. Therefore, defense proteins may protect Fusarium spp. from predators. A feature shared by all microbes producing NLPs is that they are the prey of soil fauna. Together with the observation that some NLPs act as toxins (though the toxicity has only been observed in dicot plants so far), this feature inspired the hypothesis that the biological function of NLPs maybe defense against predation. NLP members identified in Fusarium species are similar to VINLPs studied in our laboratory, therefore they appeared suitable for testing the new hypothesis.

Zymoseptoria tritici, previously known as Mycosphaerella graminicola, is a dimorphic ascomycete fungus (Quaedvlieg et al. 2011; Dean et al. 2012). It infects the leaves of wheat, causing septoria tritici blotch (STB) in both durum wheat (Triticum durum) and bread wheat (T. aestivum) (Perez-Nadales et al. 2014). Due to the importance of STB, the pathogen is very well studied. Z. tritici is an ideal model for examining the function of NLPs, because only one $N L P$-encoding gene, named $M g N L P$, was identified in its full genome sequence (Motteram et al. 2009). 
The genus Aphelenchoides belongs to the family Aphelenchoididae Skarbilovich. Most members of Aphelenchoides are fungivorous, including Aphelenchoides saprophilus.

Predators used in this study included Folsomia candida, Trichorhina tomentosa and Tenebrio molitor. F. candida belongs to the order of Collembola, which is commonly named springtails. It is a primitive arthropod, which can be found in soils worldwide. Its diet consists mainly of nematodes and fungi but also from algae and detritus (Ponge 2000). T. tomentosa, commonly known as dwarf white isopod, belongs to the order of isopoda and can be found in tropical regions in rotten wood, leaf litter and moist soil (Javidkar et al. 2015). They are used in research for testing the toxicities of chemicals in the terrestrial environment and for studies of food preference and growth (Drobne 1997), which made them an obvious choice for the following experiments.T. molitor, also known as yellow mealworm beetle, is an insect from the order of Coleoptera. They feed on various plant products, such as grain and flour, and also animal products, and can be found in granaries, mills and grain elevators as a storage pest. T. molitor is mostly found in synanthropic environments (Majka et al. 2008). For the following study, only the larvae of this species were of interest. Because worms consume the grain media in addition to fungi, this reduces the accuracy of the food choice and fitness assays (Guo et al. 2018).

\subsection{Methods and Materials}

\subsubsection{Bioinformatic analysis of VINLPs and their genes}

\section{Identification of NLP genes in Verticillium longisporum}

Malte Beinhoff (Beinhoff 2011) in his doctoral dissertation described five NLP genes from $V$. longisporum (VL43) genome, and named them as VINLP1-5. In addition, three other VINLPS are published in the VertiBase database. A total of eight VINLPS have been identified. The order of the names of VINLPs in VertiBase is not identical to Malte's dissertation. To ensure the consistency of our VINLPs project, we continued to use the names given by Malte Beinhoff (VlNLP1-5) and named the other three as VINLP6-8. The amino acid sequences of eight VINLPs were further confirmed by alignment with VdNLPs (Zhou et al. 2012). 
In addition to the genomic information available on VertiBase, we also generated our own VINLPs sequences for virulent VL43 and avirulent VL32 isolates. The CTAB method developed by Brandfass and Karlovsky (2008) was used to extract fungal DNA.The VINLPs genes were amplified using primers NO.3 to NO.15 (in 2.2.9 Primer List) by One Taq® polymerase (New England Bioline, London, UK). The PCR program considers an initial denaturation step at $94^{\circ} \mathrm{C}$ for $1 \mathrm{~min}$, followed by 30 cycles with a denaturation step at $94^{\circ} \mathrm{C}$ for $20 \mathrm{~s}$, an annealing step at $58-64^{\circ} \mathrm{C}$ for $1 \mathrm{~min}$, and an elongation step at $72^{\circ} \mathrm{C}$ for $45 \mathrm{~s}$. After isopropanol purification, the PCR products were sent for sequencing following the company's instruction (Macrogen Europe, Amsterdam, the Niederland). Afterwards our VINLPs sequences were aligned with the sequences from VertiBase, VlNLPs sequences and VaNLPs sequences (V. alfalfae VaMs.102) from EnsemblFungi database, and $V d N L P s$ sequences ( $V$. dahliae VdLs.17) from NCBI using Clustal Omega (Sievers et al. 2011) with the default setting.

\section{Phylogenetic relationships between VINLPs and NLPs of other organisms}

The amino acid sequences were predicted based on our VINLPS sequences. Collect proteins from GenBank with amino acid sequences similar to our eight VINLPs. After removing redundant results, they were aligned using Clustal Omega and analyzed by MEGA X V.10.0.4 using the neighbor-joining method (Kumar et al. 2018). A phylogenetic relationship tree was drawn with 1,000 bootstrap replications.

\subsubsection{Cytotoxicity test of VINLP1}

\section{VINLP1 Expression in Escherichia coli}

To improve the expression of VINLP1 protein in E. coli, the signal peptide predicted by SignalP 3.0 (Bendtsen et al. 2004) was removed. The coding sequence was ligated into the pET21a vector (Novagen) utilizing NdeI/BamHI via T4 ligase. The constructed pET21a_VINLP1 with a his-tag at C-terminus.

The constructs were transferred by means of heat shock and amplified in E. coli DH5 $\alpha$ cells. VLNLP1 protein production was performed in E.coli BL21 (DE3) cells, which was induced by $0.1 \mathrm{mM}$ IPTG (Isopropyl $\beta-\mathrm{D}-1$-thiogalactopyranoside). The protein induction was confirmed using SDS-PAGE. Ten microliters of the sample were loaded on the SDS-PAGE gel. The 
proteins were visualized by staining the gel with Coomassie Blue solution $(0.2 \%$ Coomassie Brilliant Blue R250, 48\% MeOH, 10\% acetic acid) for one hour and distaining the gel with $10 \%$ $\mathrm{MeOH} /$ acetic acid $(\mathrm{x} / \mathrm{x})$ for overnight. BL21 (DE3) cells carrying the pET21 vector backbone were used as control.

\section{Purification of VINLP1 protein}

The crude VINLP1 protein was purified using a cobalt ions column with Talon ${ }^{\circ}$ Metal affinity resin (Takara Bio, Japan) following the company manual. To elute the target protein, a $50 \mathrm{mM}$ of sodium phosphate elution buffer with a gradient of imidazole $(5-50 \mathrm{mM})$ consisting of $150 \mathrm{mM}$ of $\mathrm{NaCl}(\mathrm{pH}=7.0)$ was used. Purified proteins were demonstrated by SDS-PAGE.

\section{Leaf infiltration assay}

In order to test the toxicity of VINLP1 protein, $4^{\text {th }}$ leaves of 4-week-old $N$. tabacum and $4^{\text {th }}$ leaves of 4-week-old B. napus plants were infiltrated by $100 \mu \mathrm{l}$ purified VINLP1 solution at the concentration of $0.025 \mu \mathrm{g} / \mu \mathrm{l}$ respectively. Additionally, fully extended leaves of 4 -week-old Arabidopsis thaliana (Columbia-0 ecotype, Col-0) grown under short-day conditions were infiltrated as well. Necrosis caused by VINLP1 was observed after 4 days.

\subsubsection{Activation of Mitogen-activated protein kinases (MAPK)}

For transportation to Canada, the VINLP1 protein was lyophilized. $160 \mu \mathrm{l}$ of $2 \mathrm{mM}$ Hepes buffer containing $180 \mathrm{mM}$ trehalose was added into $40 \mu \mathrm{l}$ of purified VINLP1 protein. Subsequently, the mixture was immersed in liquid nitrogen and lyophilized. To assess the effect of lyophilization on the phytotoxicity activity of VINLP1, a leaf infiltration assay was performed. The lyophilized VINLP1 protein powder was dissolved in $40 \mu \mathrm{l}$ of double distilled water as stock solution. A dilution series of $0.003 \mu \mathrm{g} / \mu \mathrm{l}, 0.005 \mu \mathrm{g} / \mu \mathrm{l}, 0.0069 \mu \mathrm{g} / \mu \mathrm{l}, 0.025 \mu \mathrm{g} / \mu \mathrm{l}, 0.03 \mu \mathrm{g} / \mu \mathrm{l}, 0.05 \mu \mathrm{g} / \mu \mathrm{l}$, and $0.069 \mu \mathrm{g} / \mu \mathrm{l}$ was prepared using $2 \mathrm{mM}$ of Hepes buffer. The same dilution series of VINLP1 protein without lyophilization was used as a positive control. $2 \mathrm{mM}$ Hepes buffer was used as a negative control. One hundred microliter solution were infiltrated into $N$. tabacum leaf. Necrosis on leaves was observed after 4 days.

The bak1-4 and sobirl-1 A. thaliana mutants obtained from Prof. Zhang lab at the University of British Columbia and wild type A. thaliana Col-0 seedlings were grown on half strength 
Murashige and Skoog medium plates for twelve days. VINLP1 protein powder was dissolved in bidest water at a concentration of $0.5 \mu \mathrm{M}$ (around $13.34 \mathrm{ng} / \mu \mathrm{l}$ with the size of $26.68 \mathrm{kDa}$ ). Seedlings were collected 10 min after spraying plants with a $0.5 \mu \mathrm{M}$ of VINLP1 protein solution containing $0.01 \%$ silwet L-77. Collect untreated plants as a control. The proteins were extracted by grinding plant tissues in liquid nitrogen with protein extraction buffer ( $5 \mathrm{mM}$ of egtazic acid, 5

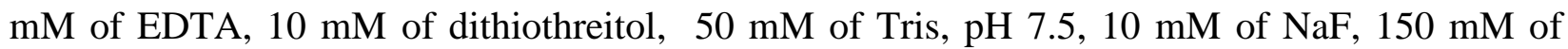
$\mathrm{NaCl}, 1 \times$ phenylmethylsulfonyl fluoride, $5 \%$ of glycerol, $1 \%$ of Triton $\mathrm{X}-100,10 \mathrm{mM}$ of $\mathrm{Na}_{3} \mathrm{VO}_{4}$, and $1 \times$ protease inhibitor). After centrifugation at full speed for $15 \mathrm{~min}$, the supernatant of protein samples was denatured together with the same volume of 2x SDS loading buffer at $99^{\circ} \mathrm{C}$ for $5 \mathrm{~min}$ and then centrifuged again at full speed for $90 \mathrm{~s}$. The protein samples were separated by SDS-PAGE after three hours of operation at $80 \mathrm{~V}$. Plant immune response was identified by western blot using antip44/42-ERK antibody (Cell Signaling, \#4370S) that recognizes the MAPK phosphorylation motif (Nitta et al. 2014).

\subsection{4 $\mathrm{V}$. longisporum strains, culture conditions and RNA extraction}

Table 1 Fungal strains used in this study

\begin{tabular}{llllllll}
\hline $\begin{array}{l}\text { Name used } \\
\text { in this study }\end{array}$ & Name & $\begin{array}{l}\text { Gö } \\
\text { Name }\end{array}$ & Species & Lineage & $\begin{array}{l}\text { Virulence } \\
\text { on crucifers }\end{array}$ & $\begin{array}{l}\text { Original } \\
\text { plant host }\end{array}$ & Geographical origin \\
\hline \multicolumn{2}{l}{ Verticillium species } & & & & & \\
VL43 & VL43 & IPP121 & V. longisporum & A1xD1 & + & Brassica napus & Mecklenburg/Germany \\
VL787 & PD644 & IPP787 & V. longisporum & A1xD1 & - & Sweden \\
VL1198 & PD730 & IPP1198 & V. longisporum & A1xD2 & - & Armoracia & Illinois/USA \\
VL1199 & PD356 & IPP1199 & V. longisporum & A1xD2 & - & Armoracia & Illinois/USA \\
VL1194 & PD589 & IPP1194 & V. longisporum & A1xD3 & + & Brassica & Gunma/ Japan \\
VL32 & VL32 & IPP0119 & V. longisporum & A1xD3 & - & Brassica napus & Mecklenburg/Germany \\
\hline
\end{tabular}

Gö Name represents the names used for fungal strains collections in Prof. A. von Tiedemann group.

+ represents virulent, - represents avirulent

Data source: Novakazi et al. (2015)

The isolates of $V$. longisporum used in this study are listed in Table 1. Six V. longisporum strains were grown in Simulated xylem fluid media (SXM) (DIXON and PEGG 1972). RNA was extracted from 14 days fungal mycelia by Trizol reagent (ambion ${ }^{\circledR}$, Thermo Fisher Scientific) 
following the company instruction. DNA was subsequently removed from RNA samples by DNase I. cDNA was then synthesized according to the manual of cDNA Synthesis Kit (New England Biolabs, Frankfurt, Germany). RT-qPCR was performed to detect the transcript levels of VINLPs with calibration by tubulin and Glyceraldehyde 3-phosphate dehydrogenase (GAPDH) gene as references. The primers involved in RT-qPCR were Primer NO.16 to NO. 24 (in 2.2.9 Primer List). CFX Manager Software (Bio-Rad) and REST 2009 software were implemented for data analysis.

\subsubsection{Vector construction and Agrobacterium-mediated transformation}

Binary vector pPK2 construction for VINLP3 and VINLP2 overexpression was performed using a Gibson assembly Master Mix from New England Biolabs (Frankfurt, Germany) according to instructions of the manufacturer. Vector pPK2 was digested at the restriction site of EcoRV. The plasmid backbone was re-assembled with the $A$. nidulans glyceraldehyde-3-phosphate dehydrogenase gpdA promoter (AN8041, A. nidulans FGSC A4), the tryptophan biosynthesis gene trpC (AN0648; A. nidulans FGSC A4) terminator and targeted VINLP3 or VINLP2 genes (Lima et al. 2009; David et al. 2008). The overlapped fragments shown on Fig. 6 were amplified by primers NO.25, NO.26, NO.27 and NO. 28 (in 2.2.9 Primer List).

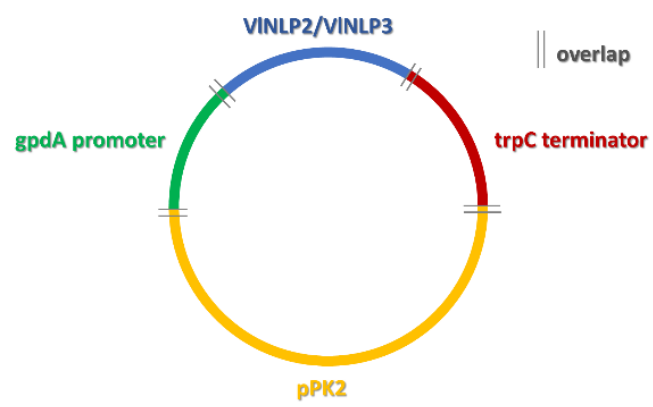

Fig. 6 The schematic figure of the re-assembled VINLP3 or VINLP2 overexpression constructs. Each pair of parallel lines represents the overlapped fragment used for Gibson assembly. Fragment NO. 1 in green is the gpdA promotor, fragment NO.2 in blue represents the target gene, fragment NO.3 in red is $\operatorname{trp} C$ terminator and the part in yellow is the pPK2 backbone.

Agrobacterium tumefaciens-mediated transformation of $V$. longisporum VL43 was performed based on the method developed by Utermark and Karlovsky (2007). The modified target genes in 
fungal isolates were further confirmed by means of PCR using primers from NO.29 to NO.32 (in 2.2.9 Primer List). Mutations were tested by sequencing the VINLP3 or VlNLP2 genes. Besides, RT-qPCR was used for verifying and quantifying the expression of target genes in transformed fungi using primers of NO. 17 and NO.18 (in 2.2.9 Primer List).

\subsubsection{Testing $V$. longisporum strains in plants}

Rapid cycling oilseed rape plants were inoculated with three VINLP3 (VINLP3_3,VINLP3_8, VINLP3_9) and three VINLP2 (VINLP2_3,VINLP2_6,VINLP2_7) overexpression VL43 strains using root dipping method described in Article II. Plants inoculated with wild type VL43 and mock inoculated with water were performed for comparison. Disease severity based on plant symptoms was assessed every 7 days till 28dpi according to the assessment key (as shown below) (Eynck et al. 2009).

\begin{tabular}{ll}
\hline Score & Symptom development \\
\hline 1 & No symptoms \\
2 & Weak symptoms on the oldest leaf (yellowing, black veins) \\
3 & Weak symptoms on the next younger leaves \\
4 & About $50 \%$ of the leaves have symptoms \\
5 & More than $50 \%$ of the leaves have symptoms \\
6 & Up to $50 \%$ of the leaves are dead \\
7 & More than $50 \%$ of the leaves are dead \\
8 & Only apex is still alive \\
9 & The plant is dead \\
\hline
\end{tabular}

Plant heights were measured at $28 \mathrm{dpi}$, subsequently quantify the fungal DNA in hypocotyl tissues by quantitative PCR (qPCR) with the primer NO.33 named as OLG 70 and OLG 71. Hypocotyl were collected, and lyophilized and pulverized. DNA was extracted from the fine powder utilizing the cetyltrimethylammonium bromide (CTAB) method developed by Brandfass and Karlovsky (2008). 


\subsubsection{Statistical analysis}

Our data analysis was performed by $\mathrm{R}$ (version 3.6.0). Shapiro-Wilk normality test was used for testing normal distribution. Afterwards, non-normal distributed data was tested by KruskalWallis test $(\mathrm{p}<0.01)$, whereas normal distributed data was tested by one-way ANOVA followed by Tukey's HSD test $(\mathrm{p}<0.01)$.

\subsubsection{Effects of NLPs on fungal predation}

\section{Fungal materials and Animals}

The beetle larvae Tenebrio molitor and the isopod Trichorhina tomentosa were purchased from Zoo \& Co. Zoo-Busch and b.t.b.e. Insektenzucht GmbH (Schnürpflingen, Germany). Folsomia candida culture (strain: Berlin) was obtained from the Institute of Zoology (University of Goettingen, Germany). Cultures of the nematodes Aphelenchoides saprophilus were obtained from Prof. Dr. Liliane Ruess (Humboldt-Universität zu Berlin, Germany). The F. graminearum strain IFA6646 (DON chemotype) was obtained from Dr. Marc Lemmens (BOKU, Tull, Austria). Wild type and two MgNLP-deficient mutants of Z. tritici were obtained from Prof. Dr. Jason J. Rudd (Centre for Sustainable Pest and Disease Management, UK).

\section{Exposure of fungi to predation}

Falcon tubes containing one rice kernel with $80 \mu \mathrm{l}$ demineralized water were autoclaved. Five microliter of $V$. longisporum (VL43) spore suspension containing around 35,000 conidia were inoculated onto rice kernels and incubated at $15^{\circ} \mathrm{C}$ in the dark. After 3-4 days, $50 \mu 1$ of nematode predators consist of 2000-3000 individuals of A. saprophilus were added to the fungal cultures. Fungal controls were incubated under the same conditions without nematodes.

The exposure of Fusarium graminearum IFA66 to springtail $F$. candida followed the method described in Article III. Transcriptomes of $V$. longisporum and F. graminearum were analyzed by RT-qPCR and RNAseq with three and four replicates, respectively.

\section{Food preference assay}

F. candida, T. tomentosa and T. molitor were involved in this assay. Gips media plates were moistened by applying $10 \mathrm{ml}$ of tap water. Two rice grains inoculated with wild type or $\triangle M g N L P$ 
mutants of Z. tritici were then positioned under sterile conditions on the media about $6 \mathrm{~cm}$ apart. Twenty randomly selected 14-day-old $F$. candida, which had been previously starved for 2 days, were released in the center of the media (Fig. 7). Afterwards, the plates were stored in a paper box to minimize the exposure to light, which was opened only during the counting process at 0.5 , $1.5,2.5,3.5,5,7,22,27$ and $45 \mathrm{~h}$ after their release on the plates. Two mutants with the same gene knock-out were performed in this study. Five replicates were used in this test.

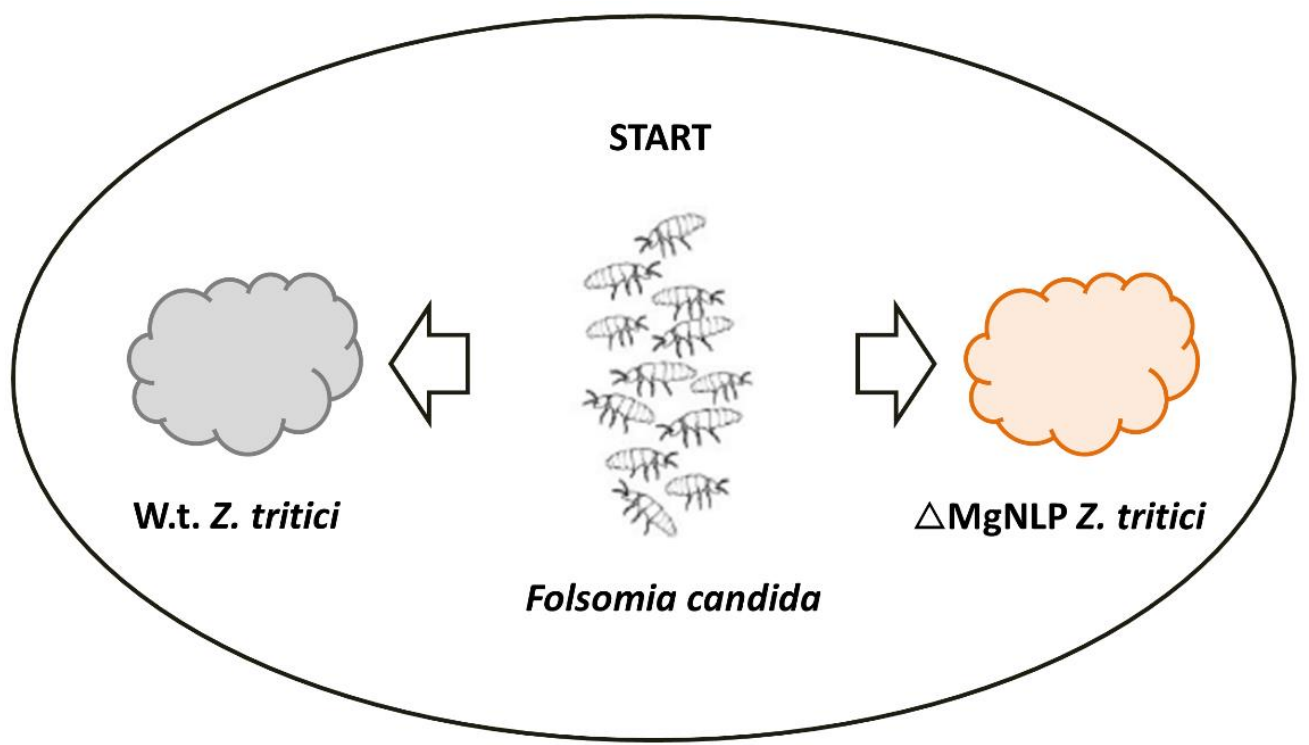

Fig. 7 Schematic diagram of food preference assay. Two inoculated rice grains with wild type or $\triangle M g N L P$ mutant of $Z$. tritici were positioned under sterile conditions on the media about $6 \mathrm{~cm}$ apart. Twenty randomly selected 14 -day-old $F$. candida were released in the center of the media. W.t., wild type; $\triangle \mathrm{NLP}, \triangle M g N L P$ mutant.

The food preference experiment of $T$. tomentosa was almost identical to the $F$. candida assay. Eight arthropods were placed on a plate and the time of counting was 1, 2, 3, 4, 5.5, 7.5, 22.5, 27.5 and $45 \mathrm{~h}$ after releasing.

As for the food preference test of $T$. molitor, eight randomly selected worms were positioned on the middle of a plate and counted after $10 \mathrm{~min}, 30 \mathrm{~min}$ and 1.5, 2.5, 5.5 and 22 hours.

\section{Fitness assay}

F. candida, T. tomentosa and T. molitor were involved in this assay. At beginning, pictures were taken for measuring animal sizes by a microscopic camera (Zeiss Stemi 305, Basler camera and software). Ten to fifteen small, randomly selected $F$. candida or five $T$. tomentosa were placed on 
gips media plates and fed by rice grains inoculated with wild type or $M g N L P$ - disrupted mutants of Z. tritici. Subsequently, the plates were sealed with parafilm and stored in a dark room at $18^{\circ} \mathrm{C}$. After four weeks, F. candida were killed by adding a piece of filter paper drenched in $50 \mu 1$ of ethyl acetate. Filter paper was removed after 30 minutes. The pictures were then analyzed by ImageJ (Version $1.51 \mathrm{k}$ ). The size of the T. tomentosa was recorded every seven days for four weeks under sterile conditions. Five replicates were conducted. Five grains inoculated with either wild type or $M g N L P$ - disrupted mutants of Z. tritici were placed on petri dishes. One T. molitor worm was placed on each plate and fifteen replicates were used in this assay. The initial weight, length and width of worm were record. Animals were stored at $25^{\circ} \mathrm{C}$. The weight of the fungi and worms, as well as the length and width of the worms was determined every seven days for three weeks.

\subsubsection{Primer List}

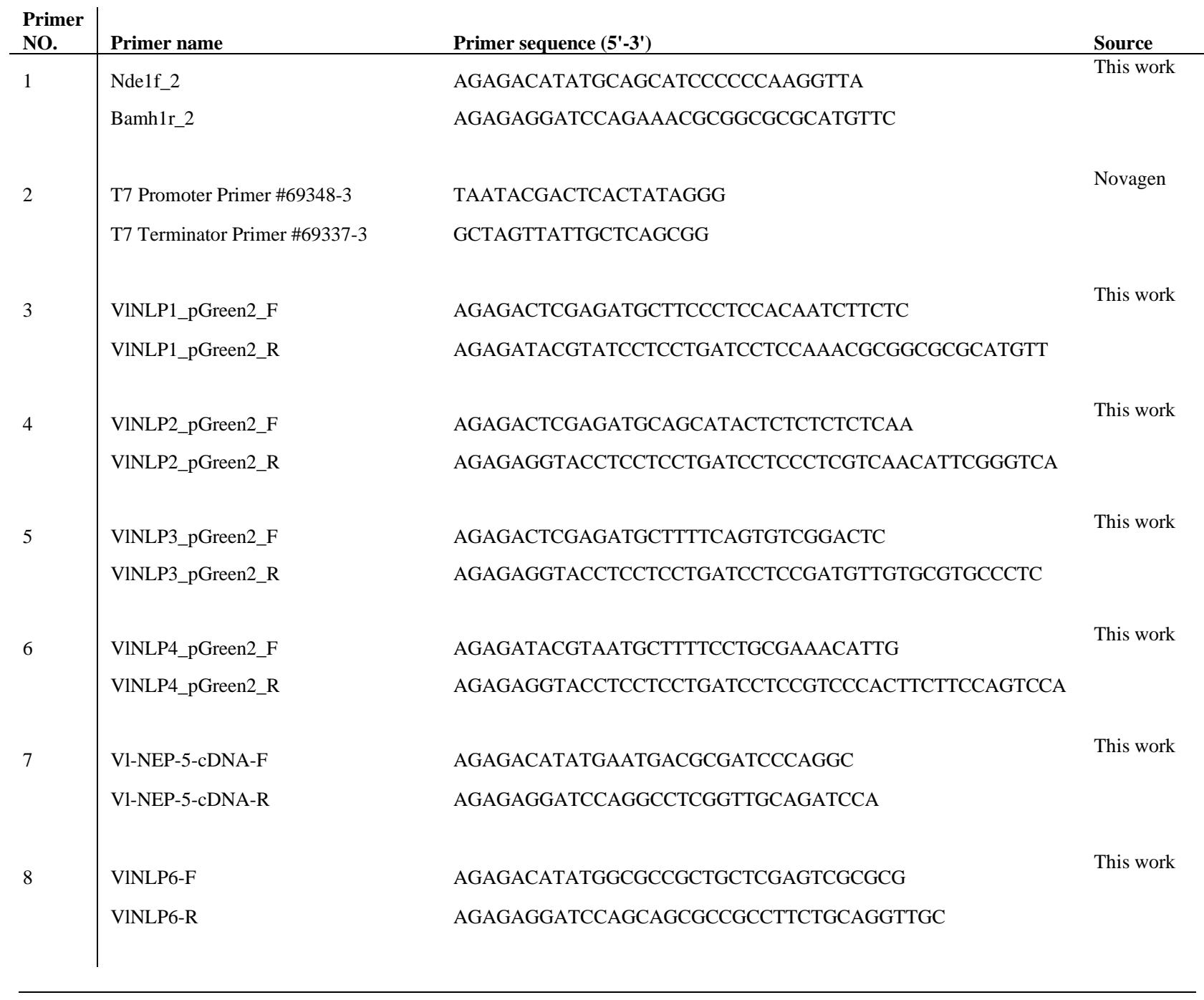




\begin{tabular}{|c|c|c|c|}
\hline \multirow[t]{2}{*}{9} & VINLP6_seq_F & CTCCTCCTCATCGTCTTCTCAT & This work \\
\hline & V1NLP6_seq_R & GATCTCCATCAGGTTCTTCACC & \\
\hline \multirow[t]{2}{*}{10} & VINLP6_seq2_F & GACGGGGAAAACCAATAACAT & This work \\
\hline & VINLP6_seq2_R & CCTCTGGTTCCGGTAAGGTAG & \\
\hline \multirow[t]{2}{*}{11} & VINLP6_seq_3_F & CCATCTGGTCTGCATCTTGTT & This work \\
\hline & VINLP6_seq_3_R1 & CATTCTTCCTCACAACCCAGA & \\
\hline \multirow[t]{2}{*}{12} & VINLP6_seq_3_F & CCATCTGGTCTGCATCTTGTT & This work \\
\hline & V1NLP6_seq_3_R2 & GAGATGTCCGTCTTCCATCT & \\
\hline \multirow[t]{2}{*}{13} & VINLP6_all_F & ATGTCGCCGTCTCTCATCAG & This work \\
\hline & VINLP6_all_R & CTACAGCGCCGCCTTCTG & \\
\hline \multirow[t]{2}{*}{14} & VINLP7-F & AGAGACATATGGGGCCTGTCAGCCTCCGGGCTGT & This work \\
\hline & VINLP7-R & AGAGAGGATCCAGGAGCGCCGCCTTGGCGAGGTT & \\
\hline \multirow[t]{2}{*}{15} & VINLP8-F & AGAGACATATGACTCCTCTGCCAGACACGCCT & This work \\
\hline & VINLP8-R & AGAGAGGATCCAGACCCCACGGCTTGAACTCGG & \\
\hline \multirow[t]{2}{*}{16} & VINLP1-qPCR-F & CCCACGGTTGCCAGCCTTAC & This work \\
\hline & VINLP1-qPCR-R & CGTTCAGGGTCATGGAGCGGG & \\
\hline \multirow[t]{2}{*}{17} & VINLP2-qPCR-F & ACAGCAATGTTTACTCCCGGC & This work \\
\hline & VINLP2-qPCR-R & ACTACGTTTTCCCAGTCGTGAC & \\
\hline \multirow[t]{2}{*}{18} & VINLP3.2_qPCR_F & CTGGGGTACTTTGGCTGGGAAA & This work \\
\hline & VINLP3.2_qPCR_R & GCCCTCGTCGTCAATGTTCTCA & \\
\hline \multirow[t]{2}{*}{19} & VINLP4-qPCR-F & ATTTGGATTCGTGGAGGTCTCGTCA & This work \\
\hline & VINLP4-qPCR-R & ATGGCCGGTAAAGTCGTCCTCATTA & \\
\hline \multirow[t]{2}{*}{20} & VINLP5-qPCR-F & GGCGACATCCAGAACACTGTGAA & This work \\
\hline & VINLP5-qPCR-R & AGTAGAATCCCTGATAAACCTTGGCA & \\
\hline \multirow[t]{2}{*}{21} & V1NLP7_qPCR_F & GACAGCGTCAAGGTCGAGTA & This work \\
\hline & VINLP7_qPCR_R & CCGAGATGGGGTACGTCTTG & \\
\hline 22 & VINLP8-qPCR-F & ACGATACGGACTCATGCTACAACAC & This work \\
\hline
\end{tabular}


VINLP8- qPCR-R

V1_ $\beta$-tubulin-F2

Vl_ $\beta$-tubulin-R2

24

25

26

27

28

29

30

31

33

\section{V1_GAPDH-F3}

V1_GAPDH-R3

VINLP3_pPK2_gibson_F1

VINLP3_pPK2_gibson_R1

VINLP3_pPK2_gibson_F2

VINLP3_pPK2_gibson_R2

VINLP3_pPK2_gibson_F3

VINLP3_pPK2_gibson_R3

VINLP2_pPK2_gibson_F2

VINLP2_pPK2_gibson_R2

pPK2-promotor_F

pPK2-promotor_R

promotor-VINLP_F

promotor-VINLP2_R

promotor-VINLP3_R

VINLP2-terminator_F

VINLP3-terminator_F

VINLP-terminator_R

terminator-pPK2_F

terminator-pPK2_R

OLG-70

OLG-71
CGCACGTAAATATTGGTCTGTTGCA

CATGATGGCCGCCTCTGAC

This work

CGAAGAGTTCTTGCTCTGGACGT

GCTCCCATCAAGGTTGGCAT

This work

GCGTACTTGGTCTCAATGAAGGG

CATGATTACGAATTCTTAATTAAGATGGTACCCGGGGATCTTTC

This work GGTGATGTCTGCTCAAGCG

GCTTGAGCAGACATCACCATGCTTTTCAGTGTCGGACTT

This work CCGGTCGGCATCTACTTCAGATGTTGTTGGTGCCCT

AGTAGATGCCGACCGGGAT

This work

GGGTACCGAGCTCGATAAAGAAGGATTACCTCTAAACAAGTG

GCTTGAGCAGACATCACCATGCAGCACACTCTCCTCTCA

This work

CCGGTCGGCATCTACT CTACTCGTCGACATTCGGGT

CTTACGAACCGAACGAGGCT

This work

TATCCTCTTGACACCGCTCC

TGCTTTGCCCGGTGTATGAA

This work

CGGCAGGGAACTCATTCGAT

CGAACCGGAAGCAATGTGTC

GTACGTGTTGCGCCATCTTG

This work

CCACGACTGGGAGCACATT

GGCGTATTGGGTGTTACGGA

CATTGGACCTCGCAACCCTA

This work

GCCAAAGGACCCTCTATGCT

CAGCGAAACGCGATATGTAG

(Eynck et al., 2007) 


\subsection{Results}

\subsubsection{Bioinformatic analysis of VINLPs and their genes}

Eight $N L P$ genes (VINLP1-8) were identified in $V$. longisporum genome

Verticillium longisporum contains eight $N L P$ genes (VlNLP1-8). Five of them (VINLP1-5) were described in Malte Beinhoff's thesis (Beinhoff 2011). In addition, VINLP6-8 are published in Vertibase. The names of VINLP gene were rearranged in this study (Table 2).

Table 2 Naming system of NLPs from V. longisporum in this study and VertiBase, and NLPs from V. dahliae

\begin{tabular}{lllllllll}
\hline This study & VINLP1 & VINLP2 & VINLP3 & VINLP4 & VINLP5 & VINLP6 & VINLP7 & VINLP8 \\
V. dahliae & $V d N L P 1$ & $V d N L P 4$ & $V d N L P 5$ & $V d N L P 9$ & $V d N L P 10$ & $V d N L P 2$ & VdNLP3 & VdNLP7 \\
VertiBase & $N L P 1$ & $N L P 4$ & $N L P 5$ & $N L P 9$ & $N L P 10$ & $N L P 2$ & $N L P 3$ & $N L P 7$ \\
\hline
\end{tabular}

\section{VINLPs sequences}

VINLPS sequences were further verified by sequencing as listed in Supplement Figure1. The primers used for sequencing were not designed for this purpose, so the amplified products only contain the entire VINLP-sequences without the up- and downstream information. The approximately $20 \mathrm{bp}$ at the beginning and the end of the sequence were modified based on the database information. In most cases, the same VINLP from different sources shared the sequences with only a slight difference, except the intron sequences in VINLP5 and VINLP7 (Suppl. Fig.1).

Unexpectedly, VINLP6 failed to be amplified using any of the primers designed based on the sequences gained from databases, including four pairs of primers (Primer NO. 9-12) located at the up- and downstream end of VINLP6 and one pair of primer (Primer NO. 13) designed from the start codon to stop codon. Therefore, VINLP6 is not involved in our following study.

\section{Putative amino acid sequences of VINLPs}

The alignment analysis based on the putative amino acid sequences of VINLPs (Fig. 8) showed that VINLP1, VINLP6 and VINLP7 are type 1; VINLP2, VINLP3, VINLP4 and VINLP8 are type 2; and VINLP5 is type 3 (Table 3). 
Article I

Table 3 Subfamilies of NLPs from $V$. longisporum

\begin{tabular}{lllllllll}
\hline VINLPs & VINLP1 & VINLP2 & VINLP3 & VINLP4 & VINLP5 & VINLP6 & VINLP7 & VINLP8 \\
NLP-Type & Type 1 & Type 2 & Type 2 & Type 2 & Type 3 & Type 1 & Type 1 & Type 2
\end{tabular}


VL43_2018_VINLP5_VdNLP10 NPP1domain

VL43_Base_VINLP6_VdNLP2

VL43_2018_VINLP1_VdNLP1

VL43_2018_VINLP7_VdNLP3

VL43_2018_VINLP4_VdNLP9

VL43_2018_VINLP8_VdNLP7

VL43_2018_VINLP2_VdNLP4

VL43_2018_VINLP3_VdNLP5

VL43_2018_V1NLP5_VdNLP10

NPP1domain

VL43_Base_VINLP6_VdNLP2

VL43_2018_VINLP1_VdNLP1

VL43_2018_VINLP7_VdNLP3

VL43_2018_VINLP4_VdNLP9

VL43_2018_VINLP8_VdNLP7

VL43_2018_VINLP2_VdNLP4

VL43_2018_VINLP3_VdNLP5

VL43_2018_VINLP5_VdNLP10

NPP1domain

VL43_Base_VINLP6_VdNLP2

VL43_2018_VINLP1_VdNLP1

VL43_2018_VINLP7_VdNLP3

VL43_2018_VINLP4_VdNLP9

VL43_2018_VINLP8_VdNLP7

VL43_2018_VINLP2_VdNLP4

VL43_2018_VINLP3_VdNLP5

VL43_2018_VINLP5_VdNLP10

NPP1domain

VL43_Base_VINLP6_VdNLP2

VL43_2018_VINLP1_VdNLP1

VL43_2018_VINLP7_VdNLP3

VL43_2018_VINLP4_VdNLP9

VL43_2018_VINLP8_VdNLP7

VL43_2018_VINLP2_VdNLP4

VL43_2018_VINLP3_VdNLP5

VL43_2018_VINLP5_VdNLP10

NPP1domain

VL43_Base_VINLP6_VdNLP2

VL43_2018_VINLP1_VdNLP1

VL43_2018_VINLP7_VdNLP3

VL43_2018_VINLP4_VdNLP9

VL43_2018_VINLP8_VdNLP7

---MY-HKILLVGVLAT-LTGLTSAN---DAIPGSAFENSGHEAA-------IAGAPMYH 45 -------MNVLTFLIAAAV---SL----AVVQADVISHDAVVPFAQPTATTTEQKAGVK 45

--MSP-SLISIVTWLAAASSTLAAP----LLESRAVINHDAVVGFPQTVPSGVSGQLMLK 53

--MLPSTIFSV----FA---LVGSA----LAQHPPKVNHDS INPVLHP--LGPNGDMIRK 45

---MVSKIFST----LASIALVAAG----PVSLRAVVPHDSLNPVKQGVQTGAFGDAIAK 49

--MLFLRNIAVVTAMVLSVPSTASVMRRQNNSSRILSESPALEPIVNG--HDFAYYFEVK 56

MTSLRTASFSAVAAL-LLLPAVIATP-LPDTPPTKLIRRDLLQPL-----GGSAWSEQEK 53

----MQHTLLSTAALLGALSAVN----A---SPAPILRRDIITAL-----PGSADEIENK 44

--MLFSVGLLALAALPSSFGAVIQARQDDPENPPRDPQPPPPGPI-----FGRAPDLDKR 53

:

F--GRSWDRKPCYPEAGQT-DGVKTDGVDSDLCFSSQNGGCADPGPWNGVNSPGNPFPVY 102

FKPQIHI-SNGCHPYPAVDANGNTSGGLKPT---GSSSAGCKGSGY--GS--------QV 91

FKPYLKV-FNGCVPFPAVNAGGDTGGGLATS---GSSNGGCSS-SA---G--------QV 97

FQPLLHI-AHGCQPYSAVNTRGEVNAGLQDS---GTTAGGCKETSK---G--------QT 90

FNPFFHI-ANGCQPYTAVKEAGDTSGGLQDS---GNISAGCRDQSK---G--------QT 94

FQPLVDFDTDSCYSVPAMTMDGTASEGLSPS----DDVGPCRPRSALDRT--------NV 104

WCPALDYDTDSCYNTVAISPSGQLNAGQDETKSGGEILGWCRKEVRLQQT--------NI 105

FQPILDFDTDGCYNTAAIDPDGNINPGKGA---TGTPQGDCRDPPQLENS--------NV 93

FQPALDFDTDSCYNVPAIGPNGDLAIGMYPF--EWPPQAGCRNEEMLDRG--------NV 103

: $\quad * \quad * \quad * \quad * \quad * \quad *$

YTVRQCNDNEWRVAYS IYYKKD---_--SGHKDWENS IVIWNGDGAGG--WKRSGT 151

YGRVATYNGVYAIMYSWYFPKDSPVTG----LGHRHDWEHVVV-WVDDIKLD-SPSIIAV 145

YARAGSYNGANAILYAWYMPKDAPSSG----LGHRHDWEGAVV-WLSSAAAD--ATVVGV 150

YARSMTLNGQFGIMYAWYWPKDQPADGNLA-SGHRHDWENVVI-WFNSNNAN-QAGILRG 147

YARAKVVNGQLAIMYSFYMPKDQPIAGNVA-GGHRHDWENIVV-FVDDPAANPAPGILGG 152

YVRGRCNRGWCAFVYAYYFQMDWAWSWPVSGYNHRHDWEHVVV-WAKEGK------VRGV 157

YVRSRCNNGWCVHMYDYYFEADFG-----W-GAHRHDWEHVAV-WVQHGQ------LKFV 152

YSGGRCNNGVCAIMYEYYFEKDQSVSGSFA-GGHRHDWENVVV-FARGDT------IVRV 145

YSRQRCNNGYCVIFYAYYFQKDTAT---PI-DGHRHDWEHIAV-WVRQSD----SFVTHV 154

$\star \quad * \star \star \star * \quad *: \star \star \quad:$ :

LLGWHSGWDYIAWGDIQNTVNNDGDLFDQG------AKDRNHAKVYQGFYYHATFSTRKT 205

SPSAHSGYNIYYPPESN------------------TIDGYSAK----VDYSSSWVVINH 182

AASAHGDFDVRPA-ADV----------------SFAGARPK----LGYRSTWP-VNH 185

AASGHGDYKKVNN---P-----------------QRNNNNLH----VEYFTSLG-KNH 180

AASGHGEYKKTAT---P----------------DREGDSVK----VEYFTTFP-TNH 185

SVSHHGGYKSRVAEDQRLRFDYTPKEFPYPAWDPMPTSVAMHPK----VVFHKDGA-RTH 212

SISQHGKWDIRILDGRTEA-------------P-RFEHGTHPK----VVYHKDGA-LTH 192

APSCHGGYGGALN----EF-------------P---VDGTSPQ----MVYHKDSA-GTH 179

AVSQHKGYDIRENSQITWT-------------A---AENGKPA----IVYHKDSI-LTH 192

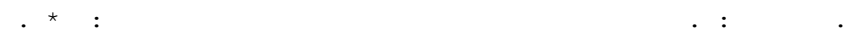




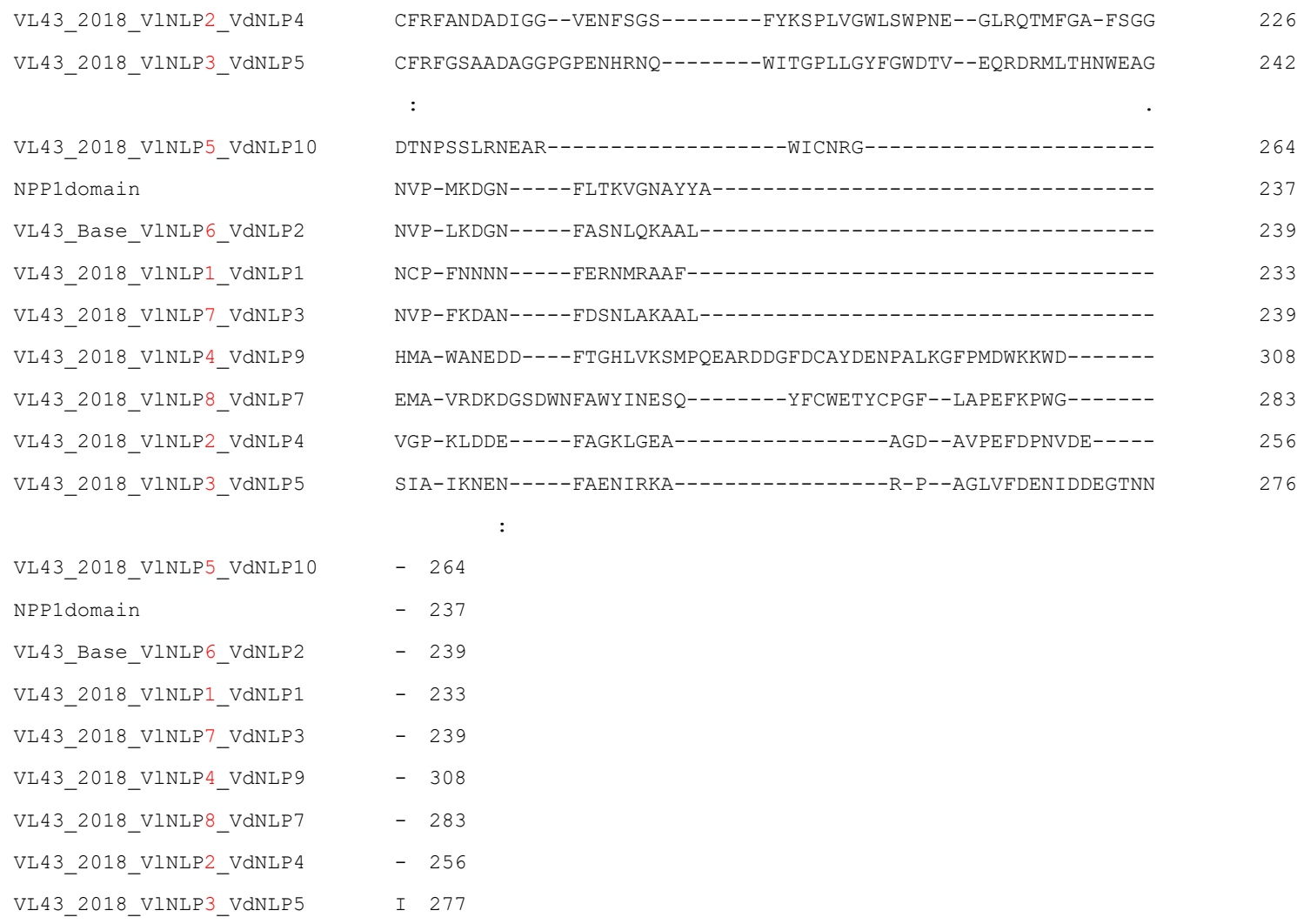

Fig. 8: Sequence alignment of putative amino acid sequences of VINLPs. Conserved features GHRHDWE and cysteine residues were mark with yellow background. Residues that are essential to its necrosis-inducing activity are in red; reduced cytotoxicity can be caused by replacing the amino acids labelled in blue.

Although all of VINLPs share the same NPP1 domain, the similarity at nucleotide level is rather low, even within the same type (Table 4).

Table 4 Similarity among VINLP sequences

\begin{tabular}{|c|c|c|c|c|c|c|c|c|c|}
\hline & V1NLP5 & NPP1 & V1NLP6 & V1NLP1 & VINLP7 & VINLP4 & V1NLP8 & V1NLP2 & V1NLP3 \\
\hline VL43_2018_V1NLP5_VdNLP10 & 100.00 & 20.00 & 21.72 & 18.32 & 21.24 & 19.30 & 17.78 & 20.20 & 19.44 \\
\hline NPP1 domain & 20.00 & 100.00 & 44.59 & 34.84 & 42.73 & 23.56 & 24.55 & 30.95 & 24.09 \\
\hline VL43_Base_VINLP6_VdNLP2 & 21.72 & 44.59 & 100.00 & 36.56 & 45.69 & 26.64 & 23.56 & 27.57 & 24.89 \\
\hline VL43_2018_VINLP1_VdNLP1 & 18.32 & 34.84 & 36.56 & 100.00 & 52.59 & 22.77 & 22.37 & 27.83 & 25.00 \\
\hline VL43_2018_V1NLP7_VdNLP3 & 21.24 & 42.73 & 45.69 & 52.59 & 100.00 & 24.23 & 23.53 & 28.37 & 25.68 \\
\hline VL43_2018_VINLP4_VdNLP9 & 19.30 & 23.56 & 26.64 & 22.77 & 24.23 & 100.00 & 34.44 & 32.26 & 36.02 \\
\hline VL43_2018_VINLP8_VdNLP7 & 17.78 & 24.55 & 23.56 & 22.37 & 23.53 & 34.44 & 100.00 & 40.41 & 36.92 \\
\hline VL43_2018_VINLP2_VdNLP4 & 20.20 & 30.95 & 27.57 & 27.83 & 28.37 & 32.26 & 40.41 & 100.00 & 39.29 \\
\hline VL43_2018_VINLP3_VdNLP5 & 19.44 & 24.09 & 24.89 & 25.00 & 25.68 & 36.02 & 36.92 & 39.29 & 100.00 \\
\hline
\end{tabular}


The alignment analysis of putative amino acid sequences between VINLPs and VdNLPs showed that the similarity of NLPs of $V$. longisporum and $V$. dahliae were more than $95 \%$, indicating that VINLPs may possess similar biochemical properties as VdNLPs (Table 5). Moreover, the different residues are mostly located out of the core part of NLP-sequence (suppl. Fig. 1).

Table 5 Similarity between NLP genes of $V$. longisporum and $V$. dahliae

\begin{tabular}{|c|c|c|c|c|c|c|c|c|}
\hline$\%$ & VdNLP1 & VdNLP4 & VdNLP5 & VdNLP9 & VdNLP10 & VdNLP2 & VdNLP3 & VdNLP7 \\
\hline $\begin{array}{l}\text { VINLP1 } \\
\end{array}$ & 96.57 & & & & & & & \\
\hline VINLP2 & & 97.27 & & & & & & \\
\hline VINLP3 & & & 98.92 & & & & & \\
\hline VINLP4 & & & & 98.38 & & & & \\
\hline VINLP5 & & & & & 100.00 & & & \\
\hline VINLP6_VertiBase & & & & & & 99.58 & & \\
\hline VINLP7 & & & & & & & 95.82 & \\
\hline VINLP8 & & & & & & & & 96.47 \\
\hline
\end{tabular}

Blastp analysis showed high identity of amino acid sequences of eight VINLPs containing proteins among various microorganism genome (Fig. 9). Overall, 56 NLPs obtained from NCBI database and eight VINLPs from present study were analyzed and clustered into three distinct groups. The classification of tested VINPs is also identically matched to the NLP-types shown in Table 3. Most of the tested NLPs belongs to type 1 and type 2. The phylogenetic results (Fig. 9) also confirmed that type 3 NLPs are more distinct than other two types. NLPs from Fusarium graminearum FGSG_03394 and FGSG_06017 are similar to VINLP2 and VINLP6, respectively, which are involved in following test related to predators. 


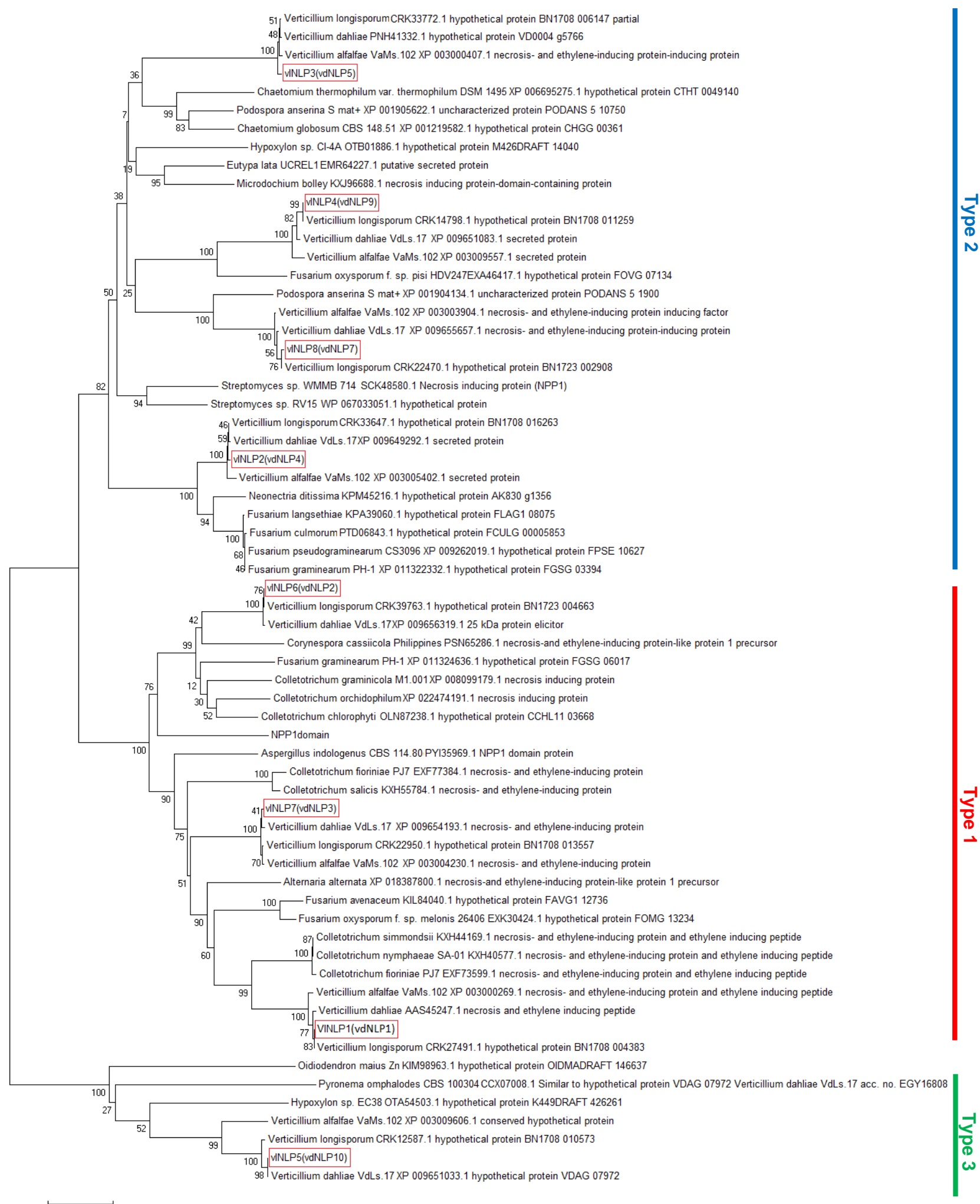

Fig. 9 Phylogenetic relationships of NLP proteins from Verticillium spp. and other microorganisms. 


\subsubsection{Necrosis-inducing activity of VINLP1}

\section{Production and purification of His-tagged VINLP1}

VINLP1 gene cloned from $V$. longisporum (VL43) was successful inserted into pET21a system containing His-tag. The insert sequence was verified utilizing sequencing.

$0.1 \mathrm{mM}$ Isopropyl- $\beta$-D-thiogalactopyranosid (IPTG) was sufficient to induce VINLP1 production in E. coli BL21(DE3) system. The SDS-PAGE gel (Fig. 10) showed that a thick band only appeared in the cells containing pET21a_VINLP1 construct after IPTG induction. Its size was also compliance with VINLP1 protein with a size of $26.68 \mathrm{kDa}$. Therefore, the production of VINLP1 protein is successful.

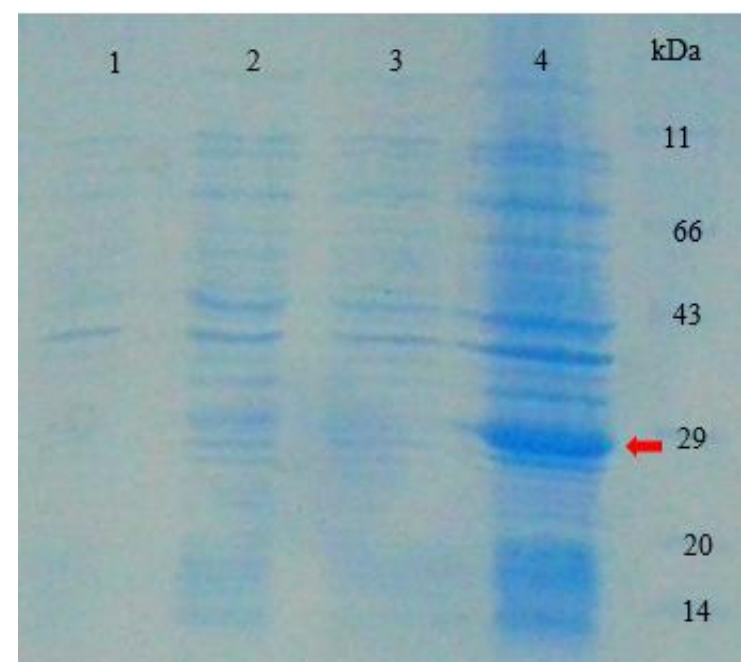

Fig.10. SDS-PAGE of VINLP1 produced in E. coli BL21 (DE3). Sample 1 is the control culture containing pET21 a vector backbone without IPTG $(0.1 \mathrm{mM})$ induction; sample 2 shows lysate of bacteria cells containing pET21a_VINLP1 construct without IPTG $(0.1 \mathrm{mM})$; sample 3 is shows control culture containing pET21a vector backbone with IPTG $(0.1 \mathrm{mM})$ induction; sample 4 shows lysate of bacterial cells containing pET21a_VINLP1 construct with IPTG $(0.1 \mathrm{mM})$ induction; the red arrow shows the expressed VINLP1 protein of approximately $27 \mathrm{kDa}$. The marker is Roti-Mark standard (Carl Roth GmbH, Karlsruhe, Germany).

As shown in the Fig. 11, produced VINLP1 protein was separated by its His-tag from all other proteins. Among the imidazole series $(5-50 \mathrm{mM})$ buffer. $30 \mathrm{mM}$ was of imidazole the optimum concentration for eluting the target VINLP1 protein from column. 


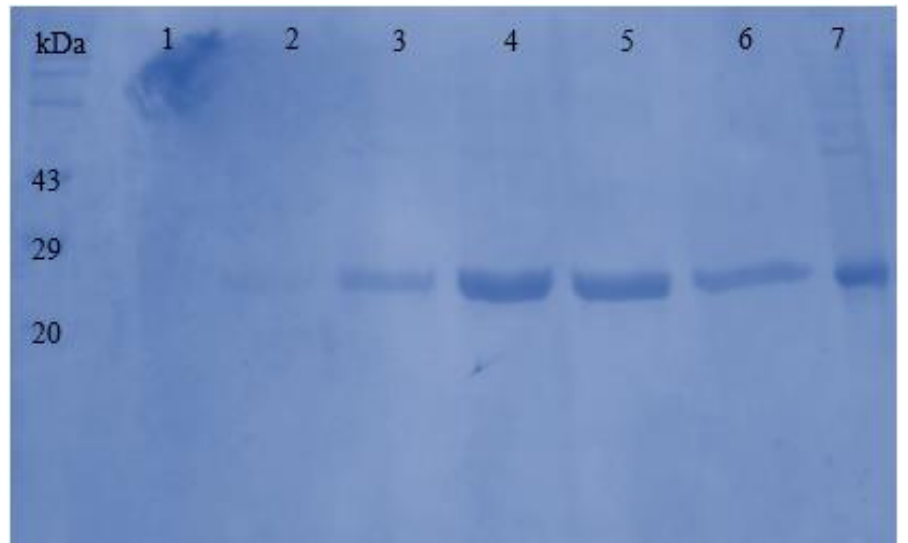

Fig.11 SDS-PAGE of His-tag purified VINLP1 protein. Sample 1 is protein eluted by $5 \mathrm{mM}$ imidazol; sample 2 protein eluted by $10 \mathrm{mM}$ imidazol; sample 3 is protein eluted by $20 \mathrm{mM}$ imidazol; sample 4 is protein eluted by $30 \mathrm{mM}$ imidazol; sample 5 is protein eluted by $40 \mathrm{mM}$ imidazol; sample 6 is protein eluted by $50 \mathrm{mM}$ imidazol; sample 7 is the unpurified VINLP1 protein; Marker is from Roti®-Mark standard (Carl Roth GmbH, Karlsruhe, Germany).

The purified protein was lyophilized into dry powder in $2 \mathrm{mM}$ of Hepes buffer containing trehalose. Both leaves infiltrated by non-lyophilized and lyophilized VINP1 showed the necrosis lesion on the leaf of Nicotiana tabacum (Fig. 12), indicating that the necrosis-inducing activity of VINP1was not affected by lyophilization. Additionally, lyophilized protein samples, which were kept at room temperature for one day, remains its high necrosis-inducing activity (data no shown).
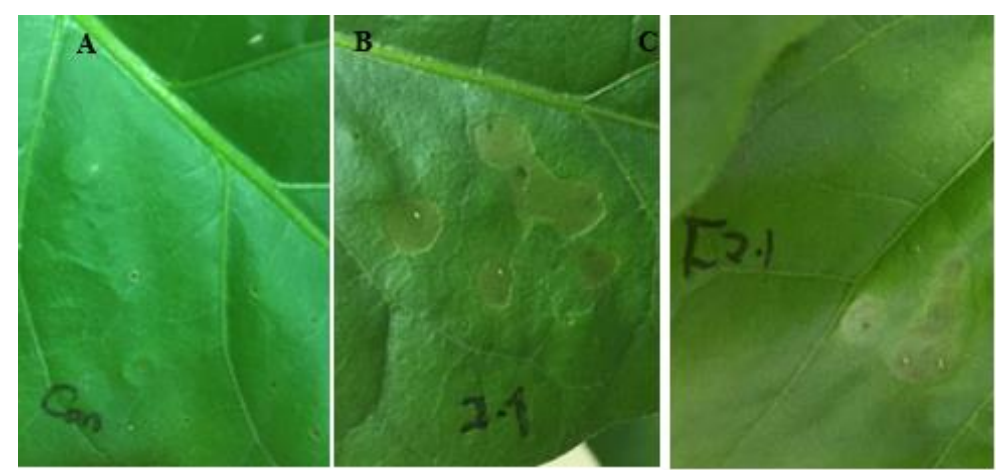

Fig. 12. Necrosis-inducing activity of VINLP1 after lyophilization. (A). Leaf of $N$. tabacum infiltrated by $100 \mu \mathrm{l}$ of $2 \mathrm{mM}$ Hepes solution; (B). Leaf of $N$. tabacum infiltrated by $100 \mu \mathrm{l}$ of $0.025 \mu \mathrm{g} / \mu \mathrm{l}$ nonlyophilized VINP1 protein solution; (C). Leaf of $N$. tabacum infiltrated by $100 \mu \mathrm{l}$ of $0.025 \mu \mathrm{g} / \mu \mathrm{l}$ lyophilized VINLP1 protein solution. 
Three different dicotyledons, N. tabacum, B. napus and A. thaliana (Col-0) have showed visible lesions on leaves after infiltrated by $0.025 \mu \mathrm{g} / \mu \mathrm{l}$ of purified protein (Fig. 13).
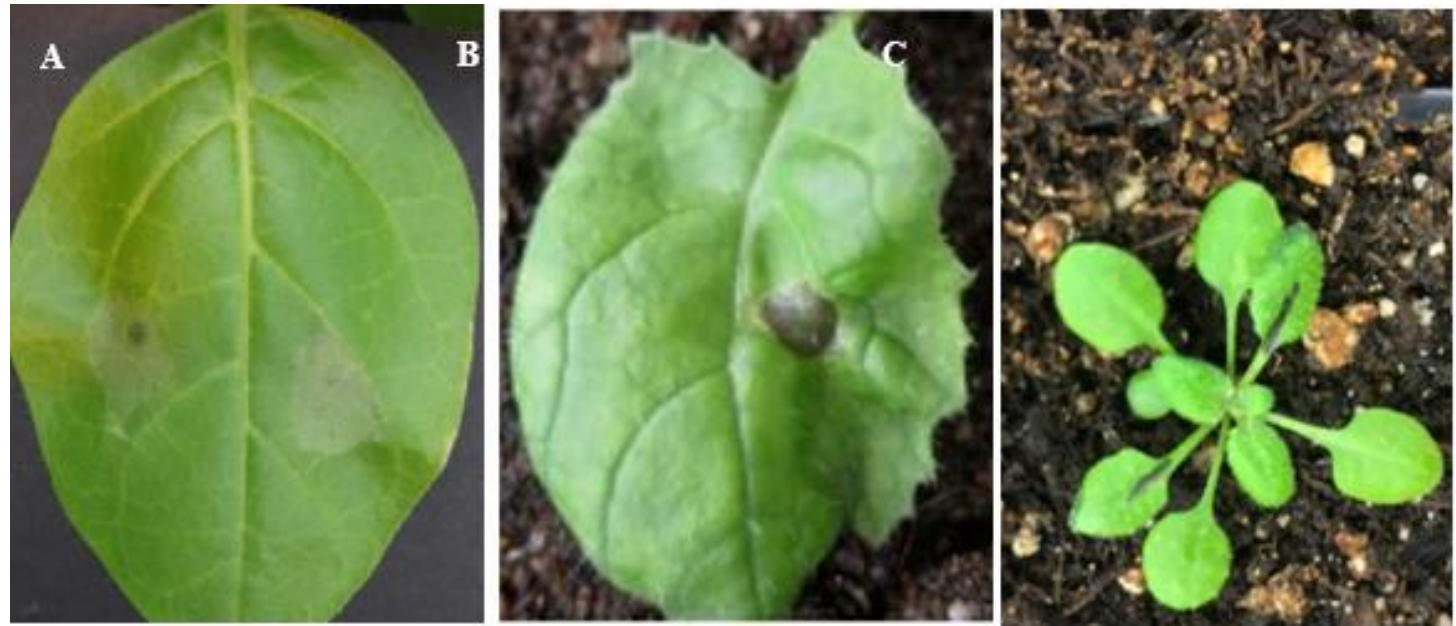

Fig. 13 Leaf infiltration assay for necrosis-inducing activity of VINLP1. (A). Necrosis lesions on leaf of $N$. tabacum infiltrated by $100 \mu \mathrm{l}$ of $0.025 \mu \mathrm{g} / \mu \mathrm{l}$ non-lyophilized VlNP1 protein solution after 24h; (B). Necrosis lesions on leaf of $B$. napus infiltrated by $100 \mu \mathrm{l}$ of $0.025 \mu \mathrm{g} / \mu \mathrm{l}$ non-lyophilized VlNP1 protein solution after 48h; (C). Necrosis lesions on leaf of $A$. thaliana Col-0 infiltrated by $100 \mu \mathrm{l}$ of $0.025 \mu \mathrm{g} / \mu \mathrm{l}$ lyophilized VINLP1 protein solution.

\subsubsection{Plant immune responses caused by VINLP1}

Böhm et al. (2014b) reported a pattern of 20 amino acid residues (nlp20), which is able to trigger plant defense and immunity in Arabidopsis. The amino acid sequence of VINLP1 includes 20 residues with 70 percent sequence similarity with published nlp20.

An RLP23-SOBIR1-BAK1 complex was published to confer nlp20 recognition and mediate NLP-triggered immunity in Arabidopsis (Albert et al. 2015). In addition to assessing whether VINLP1 is able to trigger plant immune responses, the associated receptor SOBIR1 and BAK1 was also tested based on MAPK activation detection. 


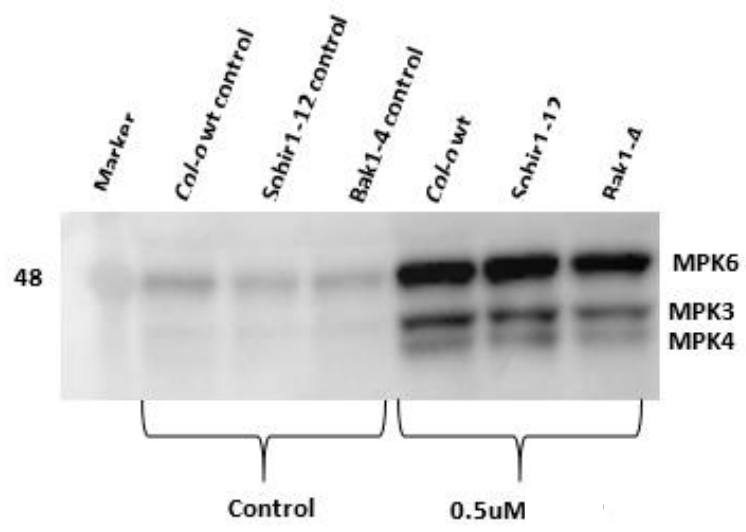

Fig.14 VINLP1 protein induced MAPK activation in wild type, sobir1-12 and bak1-4 of $A$. thaliana Col-0. $0.5 \mu \mathrm{M}$ VINLP1 solution sprayed on 12-day-old seedlings grown on $1 / 2 \mathrm{MS}$ medium for $10 \mathrm{~min}$. The control plants had no any treatment before sampling. The a-p44/42-ERK antibody (Cell Signaling Technology, Inc., \#4370s) was used for recognizing a conserved phosphorylation motif of MAP kinases of extracted protein in a western blot.

MAP kinase activation was increased in wild type and sobirl or bakl deletion Col-0 plants treated with VINLP1 solution, showing that RLP23-SOBIR1-BAK may not a requirement for VINLP1 protein recognition and plant immune responses induction. However, the complete VINLP1 protein is toxic to plants and able to cause necrosis on plants (Fig. 12). The plant immune responses may be caused by damage. So this immunoblot responses cannot indicate whether the receptor of VINLP1 is RLP23-SOBIR1-BAK1 in Arabidopsis. But it can be inferred by sequences comparison. The amino acid sequence of nlp20(VINLP1) with 70 percent sequence similarity with published nlp20 (Böhm et al. 2014b). Nlp20 (FoNLP) from F. oxysporum can trigger pattern-induced immunity in Arabidopsis. Our nlp20 (VINLP1) is highly identical to nlp20 (FoNLP) as shown in Table 6, except $A_{1} G$ and $V_{19} A$ that are not essential to trigger pattern-induced immunity in Arabidopsis. So it is very likely that nlp20(VINLP1) can also works as typical PTI triggering pattern, even can be recognized by RLP23-SOBIR1-BAK1. Although nlp20(V1NLP6) has a less similarity with nlp20 (FoNLP) compared to nlp20(V1NLP1), it contains four amino acids $\left(\mathrm{I}_{2}, \mathrm{Y}_{4}, \mathrm{~W}_{6}\right.$ and $\left.\mathrm{Y}_{7}\right)$ that are essential to trigger pattern-induced immunity in Arabidopsis as well (Table 6). 
Table 6. Eight nlp20 orthologous peptides from $V$. longisporum

\begin{tabular}{|c|c|c|c|c|c|c|c|c|c|c|c|c|c|c|c|c|c|c|c|c|c|c|c|c|c|c|c|c|c|c|c|}
\hline Name & ETI & Protein & NLP & & & & & & & & & & & ninc & $\mathrm{oac}$ & id s & equ & ienc & ce of & $\mathrm{nlp}$ & 20 & & & & & & & & & & \\
\hline nlp20 (ref.) & Yes & Yes & Type 1 & $A$ & 1 & $M$ & $\overline{\mathrm{Y}}$ & $\mathrm{S}$ & $\bar{M}$ & \begin{tabular}{|l|l|}
$Y$ & \\
\end{tabular} & $\mathrm{~F}$ & $P$ & $\mathrm{~K}$ & $\mathrm{D}$ & $\mathrm{S}$ & $\mathrm{P}$ & V & $\mathrm{T}$ & $G$ & - & - & $\mathrm{L}$ & - & - & $\mathrm{G}$ & $\mathrm{H}$ & $R$ & $\mathrm{H}$ & D & W & $E$ \\
\hline nlp20 (FoNLP) & Yes & Yes & Type 1 & A & 1 & $M$ & $\mathrm{Y}$ & A & w & $\mathrm{Y}$ & w & $P$ & $\mathrm{~K}$ & $\mathrm{D}$ & Q & $P$ & $A$ & $\mathrm{D}$ & G & - & $\mathrm{N}$ & $\mathrm{L}$ & V & $S$ & G & $\mathrm{H}$ & $\mathrm{R}$ & H & D & w & $\mathrm{E}$ \\
\hline nlp20 (VINLP1) & Yes & Yes & Type 1 & G & 1 & $\mathrm{M}$ & $\mathrm{Y}$ & A & W & $\mathrm{Y}$ & W & $\mathrm{P}$ & $\mathrm{K}$ & D & $\mathrm{Q}$ & $\mathrm{P}$ & A & $\mathrm{D}$ & G & - & $\mathrm{N}$ & $\mathrm{L}$ & A & $\mathrm{S}$ & G & $\mathrm{H}$ & $\mathrm{R}$ & $\mathrm{H}$ & D & W & $E$ \\
\hline nlp20 (VINLP2) & No & No & Type 2 & A & 1 & $\mathrm{M}$ & $\mathrm{Y}$ & $E$ & $\mathrm{Y}$ & $\mathrm{Y}$ & $\mathrm{F}$ & $\mathrm{E}$ & $\mathrm{K}$ & $\mathrm{D}$ & $Q$ & $S$ & V & $\mathrm{S}$ & G & - & $\mathrm{S}$ & $\mathrm{F}$ & A & G & G & $\mathrm{H}$ & $\mathrm{R}$ & H & D & W & $E$ \\
\hline nlp20 (VINLP3) & No & No & Type 2 & V & 1 & $\mathrm{~F}$ & $\mathrm{Y}$ & $A$ & $\mathrm{Y}$ & $\mathrm{Y}$ & $\mathrm{F}$ & $\mathrm{Q}$ & $\mathrm{K}$ & $\mathrm{D}$ & $\mathrm{T}$ & - & $A$ & $\mathrm{~T}$ & $\mathrm{P}$ & - & - & 1 & $\mathrm{D}$ & - & G & $\mathrm{H}$ & $\mathrm{R}$ & H & D & W & $E$ \\
\hline nlp20 (VINLP4) & No & No & Type 2 & A & $\mathrm{F}$ & V & $\mathrm{Y}$ & A & $\mathrm{Y}$ & $\mathrm{Y}$ & $\mathrm{F}$ & $\mathrm{Q}$ & M & $\mathrm{D}$ & W & A & W & $\mathrm{S}$ & W & $\mathrm{P}$ & V & $\mathrm{S}$ & $S$ & $Y$ & $\mathrm{~N}$ & $\mathrm{H}$ & $\mathrm{R}$ & $\mathrm{H}$ & D & W & $E$ \\
\hline nlp20 (VINLP5) & No & No & Type 3 & $\mathrm{R}$ & $\mathrm{V}$ & A & $\mathrm{Y}$ & $\mathrm{S}$ & I & $\mathrm{Y}$ & $Y$ & K & $\mathrm{K}$ & $\mathrm{D}$ & $S$ & - & - & - & - & - & - & - & - & - & G & $\mathrm{H}$ & $\mathrm{K}$ & $N$ & D & W & $E$ \\
\hline nlp20 (VINLP6) & Yes & Yes & Type 1 & A & 1 & $\mathrm{~L}$ & $\mathrm{Y}$ & A & $w$ & $\mathrm{Y}$ & M & $\mathrm{P}$ & $\mathrm{K}$ & D & $A$ & $\mathrm{P}$ & S & $\mathrm{S}$ & G & & & $\mathrm{L}$ & & & G & $\mathrm{H}$ & $\mathrm{R}$ & $\mathrm{H}$ & D & W & $E$ \\
\hline nlp20 (VINLP7) & No & No & Type 1 & A & 1 & $M$ & $\mathrm{Y}$ & $\mathrm{S}$ & $F$ & $\mathrm{Y}$ & M & $\mathrm{P}$ & $\mathrm{K}$ & D & $\mathrm{Q}$ & $\mathrm{P}$ & I & A & G & & $\mathrm{N}$ & V & A & G & G & $\mathrm{H}$ & $\mathrm{R}$ & $\mathrm{H}$ & D & W & $E$ \\
\hline nlp20 (VINLP8) & No & No & Type 2 & $\mathrm{~V}$ & $\mathrm{H}$ & $\mathrm{M}$ & $Y$ & $\mathrm{D}$ & $\mathrm{Y}$ & $Y$ & $\mathrm{~F}$ & $\mathrm{E}$ & A & $\mathrm{D}$ & $\mathrm{F}$ & & & & G & W & & & & G & A & $\mathrm{H}$ & $\mathrm{R}$ & $\mathrm{H}$ & D & W & $\mathrm{E}$ \\
\hline
\end{tabular}

nlp20(ref.) is the reference nlp20 sequence from Phytophthora parasitica (Böhm et al. 2014b); nlp20 (V1NLP1-8) are from Verticillium longisporum (this work); nlp20 (FoNLP) is from Fusarium oxysporum. (Böhm et al. 2014b) Red boxes represent four residues essential to trigger pattern-induced immunity in Arabidopsis (Böhm et al. 2014b). The residue difference between nlp20(V1NLP1) and nlp20(FoNLP) are labeled with yellow background. The ETI capability and protein toxicity of nlp20(V1NLPs) were predicted based on Böhm et al. $2014 \mathrm{~b}$ and Zhou et al. 2012, respectively.

Amino acid sequences comparison shows that triggering pattern-induced immunity is independent from necrosis-inducing activity (Table 7). HaNLP3 from Hyaloperonospora arabidopsidis is not able to induce necrosis on leaves, but its nlp20 triggered plant immunity successfully. In contrast, NLPpcc from Pectobacterium carotovorum pv. Carotovorum has necrosis-inducing acitivity, but its nlp20 does not contain four essential amino acids (Böhm et al. 2014b; Oome et al. 2014; Zhou et al. 2012).

Table 7 Nlp20 orthologous peptides from cytotoxic and non-cytotoxic NLP proteins

\begin{tabular}{|c|c|c|c|c|c|c|c|c|c|c|c|c|c|c|c|c|c|c|c|c|c|c|c|c|c|c|c|c|c|c|c|}
\hline Name & ETI & Protein & NLP & & & & & & & & & & Am & ino & aci & id & sequ & uen & ce o & of $n$ & Ip2C & & & & & & & & & & \\
\hline nlp20(ref.) & Yes & Yes & Type 1 & A & 1 & $M$ & $\mathrm{Y}$ & $\mathrm{S}$ & $w$ & $Y$ & $\mathrm{~F}$ & $P$ & $\mathrm{~K}$ & $\mathrm{D}$ & $\mathrm{S}$ & $\mathrm{P}$ & $\mathrm{V}$ & $T$ & G & - & - & $\mathrm{L}$ & - & - & G & $\mathrm{H}$ & $\mathrm{R}$ & $\mathrm{H}$ & $\mathrm{D}$ & $\mathrm{W}$ & $E$ \\
\hline nlp20 (HaNLP3) & Yes & No & Type 1 & $A$ & 1 & $M$ & $\mathrm{Y}$ & $A$ & $w$ & $\mathrm{Y}$ & $\mathrm{F}$ & $P$ & $\mathrm{~K}$ & D & $\mathrm{S}$ & $\mathrm{P}$ & $M$ & - & - & - & - & L & $\mathrm{L}$ & M & G & $\mathrm{H}$ & $\mathrm{R}$ & $\mathrm{H}$ & $\mathrm{D}$ & W & $E$ \\
\hline nlp20 (NLP $\left.{ }_{p c c}\right)$ & No & Yes & Type 2 & G & $\mathrm{s}$ & $\mathrm{F}$ & $\mathrm{Y}$ & $A$ & L & $\mathrm{Y}$ & $\mathrm{F}$ & $\mathrm{L}$ & $\mathrm{K}$ & $\mathrm{D}$ & Q & I & L & $\mathrm{N}$ & G & V & $\mathrm{N}$ & & & $S$ & G & $\mathrm{H}$ & $\mathrm{R}$ & $\mathrm{H}$ & $\mathrm{D}$ & W & $E$ \\
\hline
\end{tabular}

$\mathrm{Nlp} 20$ (ref) is the reference nlp20 sequence from Phytophthora parasitica (Böhm et al. 2014b); nlp20 (HaNLP3) is from Hyaloperonospora arabidopsidis (Oome et al. 2014); nlp20 (NLPpcc) is from Pectobacterium carotovorum pv. carotovorum (Oome et al. 2014, Mattinen et al. 2004); Red box represents four residues essential to trigger pattern-induced immunity in Arabidopsis. 


\subsubsection{VlNLPs expression in various Verticillium isolates}

Beinhoff (2011) pointed that not all of VINLPs are expressed in VL43. However, whether this VINLP gene suppression was specific to VL43 was not known. Six V. longisporum strains (Table 1) belonging to three lineages were selected for the study of the expression of VlNLP genes, including strains virulent and avirulent on oilseed rape (Novakazi et al. 2015). Due to conflicts of the sequence of VINLP6 that we generated with the sequence in VertiBase, VINLP6 was not included in this study.

The analysis of seven VINLPS transcript levels demonstrated that VINLP3 was the only gene expressed below the detectable level in VL43. Unexpectedly, VINLP3 was expressed in three out of six $V$. longisporum strains. As summarized in Table 8, lineage A1/D2 was not able to express VINLP3, while the avirulent strain expressed more VINLP3 than virulent strains in both lineage A1/D1 and A1/D3 (Fig. 15). Our preliminary assumption is that expression of VlNLP3 contributes to avirulence in $V$. longisporum. In another word, suppression of $V l N L P 3$ is prerequisite for the adaptation to pathogens.

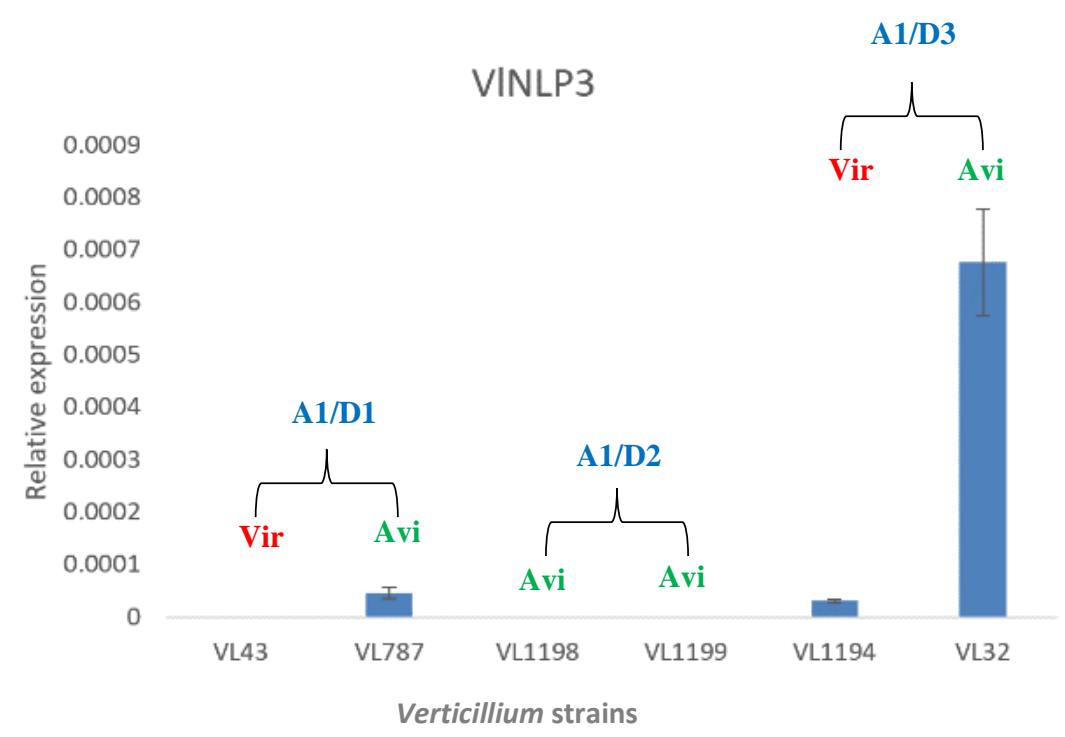

Fig. 15 VlNLP3 expression in $V$. longisporum strains. RT-qPCR was performed for this $V l N L P s$ transcript level analysis. The abbreviation "Vir" in red represents the oilseed rape virulent strain; "Avir" in green is the avirulent; A1/D1, A1/D2 and A1/D3 are the three lineages. The relative expression represents the transcript level with modification using reference gene GAPDH (Pfaffl 2001). Bars are the mean of tests conducted in triplicate \pm standard error. 
Table 8 Expression of $V I N L P$ s genes in various $V$. longisporum isolates

\begin{tabular}{|c|c|c|c|c|c|c|c|c|c|c|}
\hline & & & \multicolumn{8}{|c|}{ Gene expression } \\
\hline & & & \multicolumn{2}{|l|}{ A1/D1 } & \multicolumn{2}{|l|}{ A1/D2 } & \multicolumn{4}{|l|}{ A1/D3 } \\
\hline & & & Vir & Avir & Avir & Avir & Vir & Avir & & \\
\hline Toxicity & $\begin{array}{l}\text { NLP } \\
\text { Type }\end{array}$ & V. longisporum & $V L 43$ & $V L 787$ & VL1198 & VL1199 & VL1194 & $V L 32$ & V. dahliae & $\begin{array}{l}\text { Gene } \\
\text { expression }\end{array}$ \\
\hline Yes & Type1 & $V I N L P 1$ & + & ++ & +++++ & + & + & ++ & $V d N L P 1$ & + \\
\hline No & Type2 & $V I N L P 2$ & + & + & + & + & + & + & $V d N L P 4$ & + \\
\hline No & Type2 & VINLP3 & $\mathbf{x}$ & + & $\mathbf{x}$ & $\mathbf{x}$ & + & ++ & VdNLP5 & $\mathrm{x}$ \\
\hline No & Type2 & VINLP4 & + & + & + & + & + & + & $V d N L P 9$ & + \\
\hline No & Type3 & VINLP5 & + & + & + & + & + & + & $V d N L P 10$ & + \\
\hline No & Type1 & VINLP7 & + & + & + & + & + & + & $V d N L P 3$ & + \\
\hline No & Type2 & VINLP8 & + & + & + & + & + & + & $V d N L P 7$ & + \\
\hline
\end{tabular}

The comparison of each VlNLP-gene transcript level among six $V$. longisporum strains is summarized in figure 16. Apart from VINLP3, other six VINLPS were expressed in all tested strains. The highest expression of VINLPl was detected in the virulent isolate VL1194, while the avirulent isolate VL32 has the highest transcript level of most of non-cytotoxic VlNLPs, including $V I N L P 2, V L N L P 3, V I N L P 4$ and $V I N L P 7$. These results suggest that VINLPs expression level may not correlate with fungal virulence.

The comparison of seven VINLPs transcript levels in each V. longisporum strain is concluded in Figure 17. VlNLP1 is the highest expressed NLP gene in most strains, except VINLP2 in VL1198. But another avirulent strain VL1199 from the same lineage (A1/D2) as VL1198 expressed more VINLP1. The comparison revealed that VINLP1 is the most important VlNLP. In addition, $V I N L P 2$ may play essential role under certain unknown condition. 

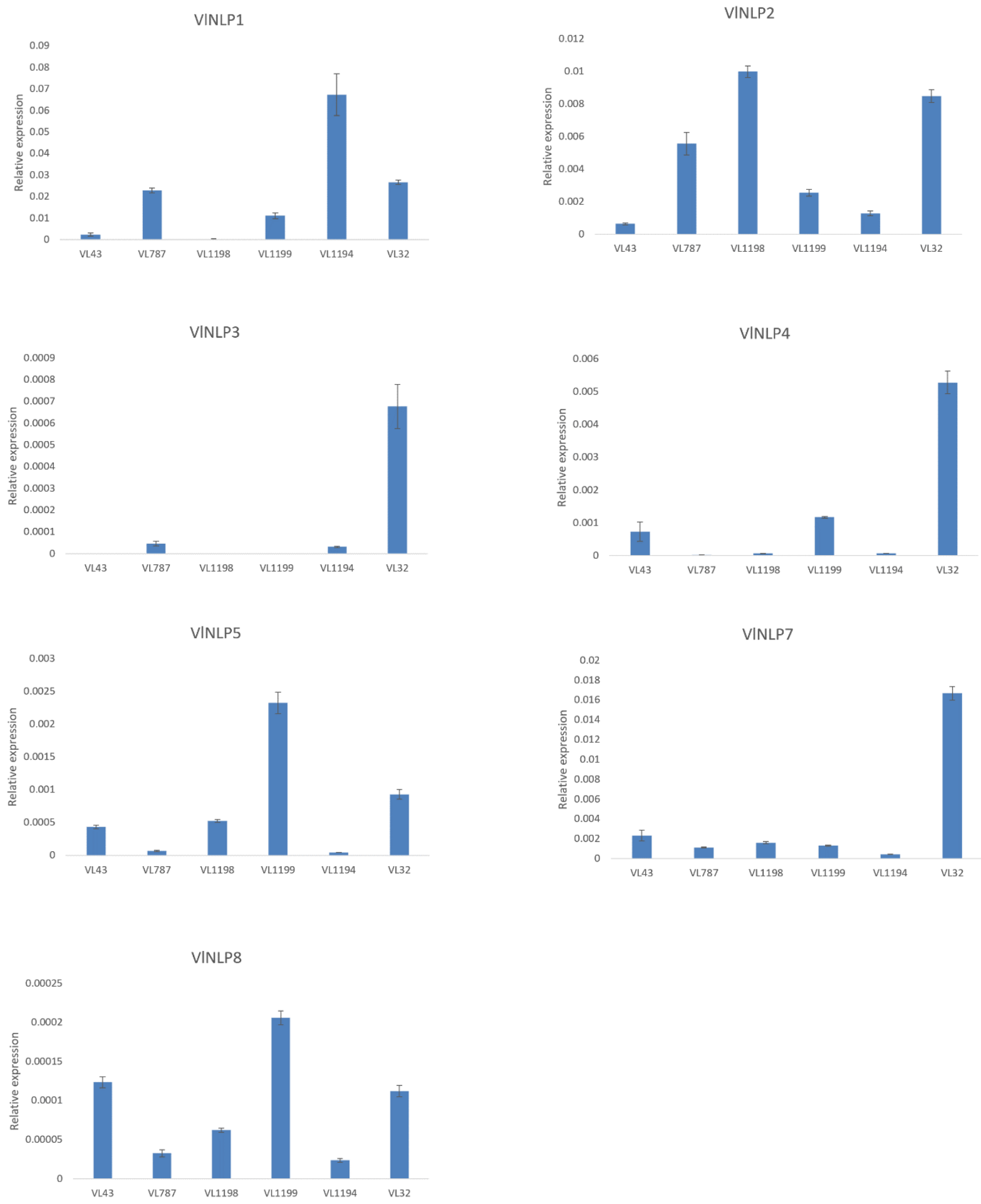

Fig. 16 Comparison of transcript level of each VINLP among various $V$. longisporum isolates. The relative expression represents the transcript level with the modification using reference gene GAPDH. Bars are the mean of tests conducted in triplicate \pm standard error. 

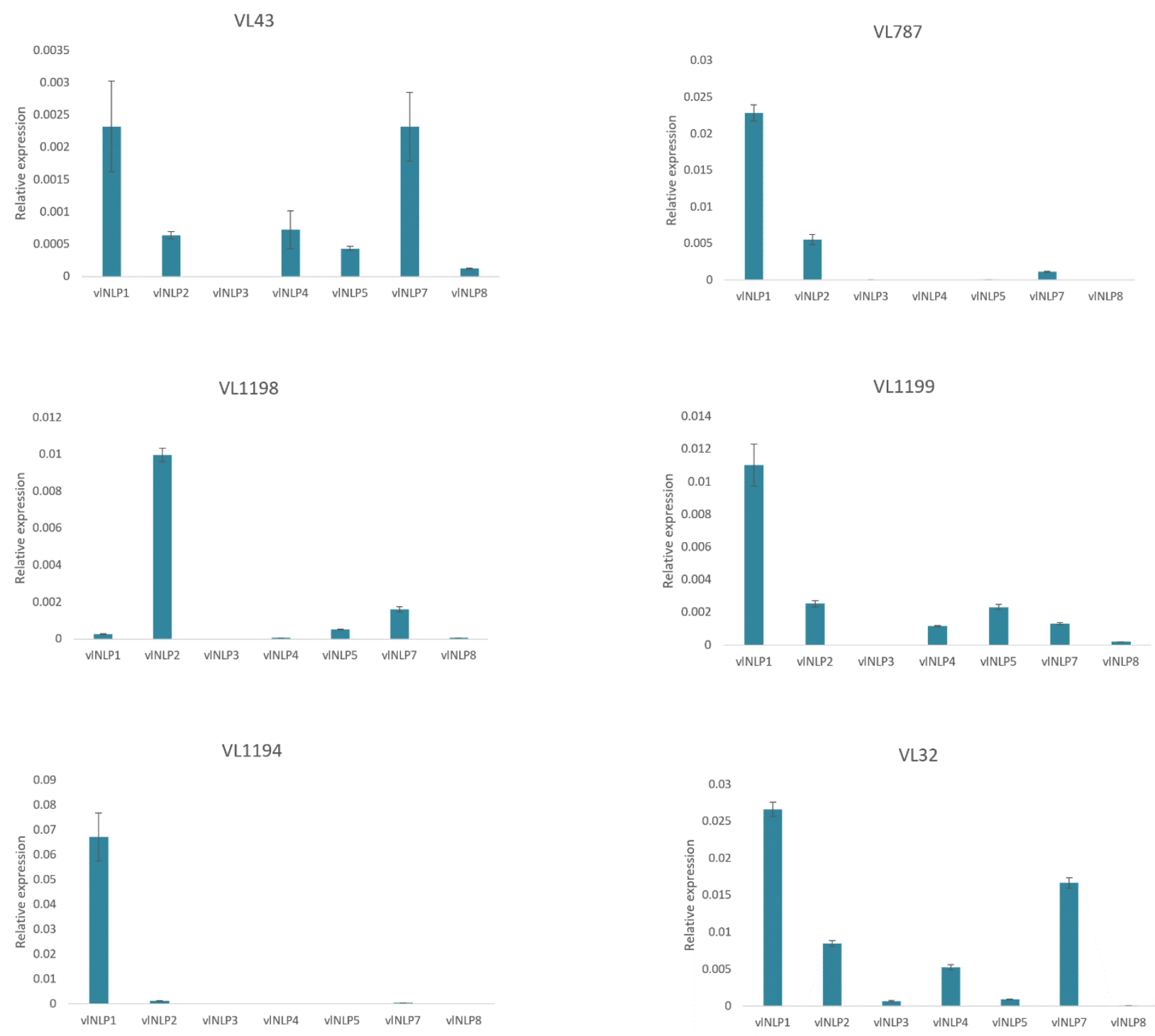

Fig.17 Comparison of transcript levels of seven VINLPs in each $V$. longisporum strain. The relative expression represents the transcript level with modification using reference gene $G A P D H$. Bars are the mean of tests conducted in triplicate \pm standard error. 


\subsubsection{Overexpression of $V I N L P 3$ and $V I N L P 2$ in $V$. longisporum VL43}

The following experiments were performed to test our assumption that the suppression of VINLP3 was a prerequisite for the adaptation of the pathogen to crucifers. VL43 mutant strain overexpressing VINLP3 was constructed. A root-dipping plant infection experiment was used to find out whether VINLP3 expression reduced the virulence of the pathogen. Because the sequence of VINLP2 gene was very similar to VINLP3, strains overexpressing VINLP2 were constructed for a comparison.

The modified VL43 strains showed overexpression of VINLP3 (VINLP3_3, VINLP3_8, VINLP3_9) and VINLP2 (VINLP2_3, VINLP2_6, VINLP2_7) (Fig. 18 and 20). The strains VINLP3_8 and VINLP2_3 have the highest expression of corresponding genes. Addition to the target genes, VINLP1 expression was also up-regulated in both overexpression lines (Fig. 19 and 21). This may indicate an interaction between NLP-members.

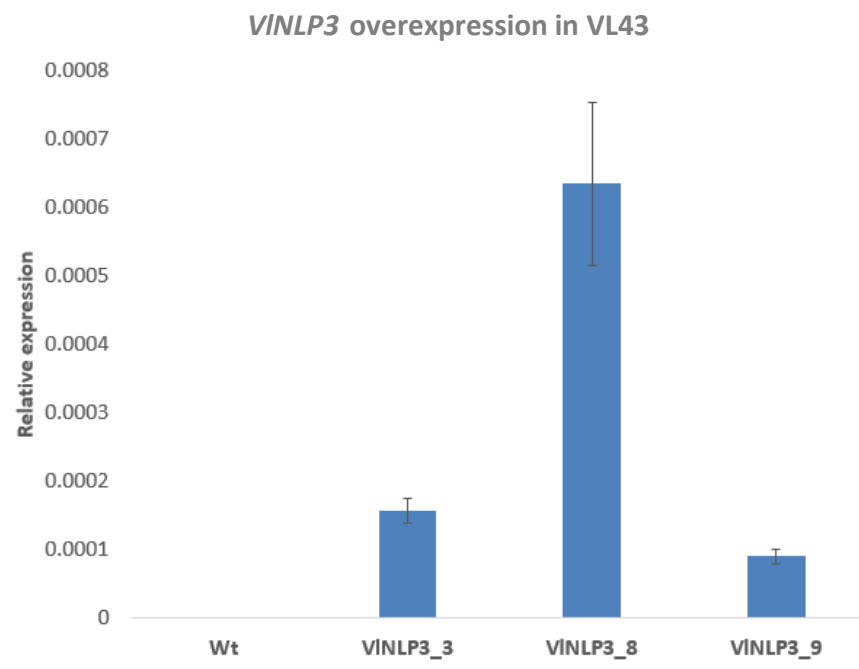

Fig. 18 Expression levels of $V I N L P 3$ in wild type and three VINLP3 overexpression mutants of $V$. longisporum VL43. The relative expression represents the transcript level with modification using reference gene $G A P D H$. Bars represent the mean of tests conducted in triplicate \pm standard error. 


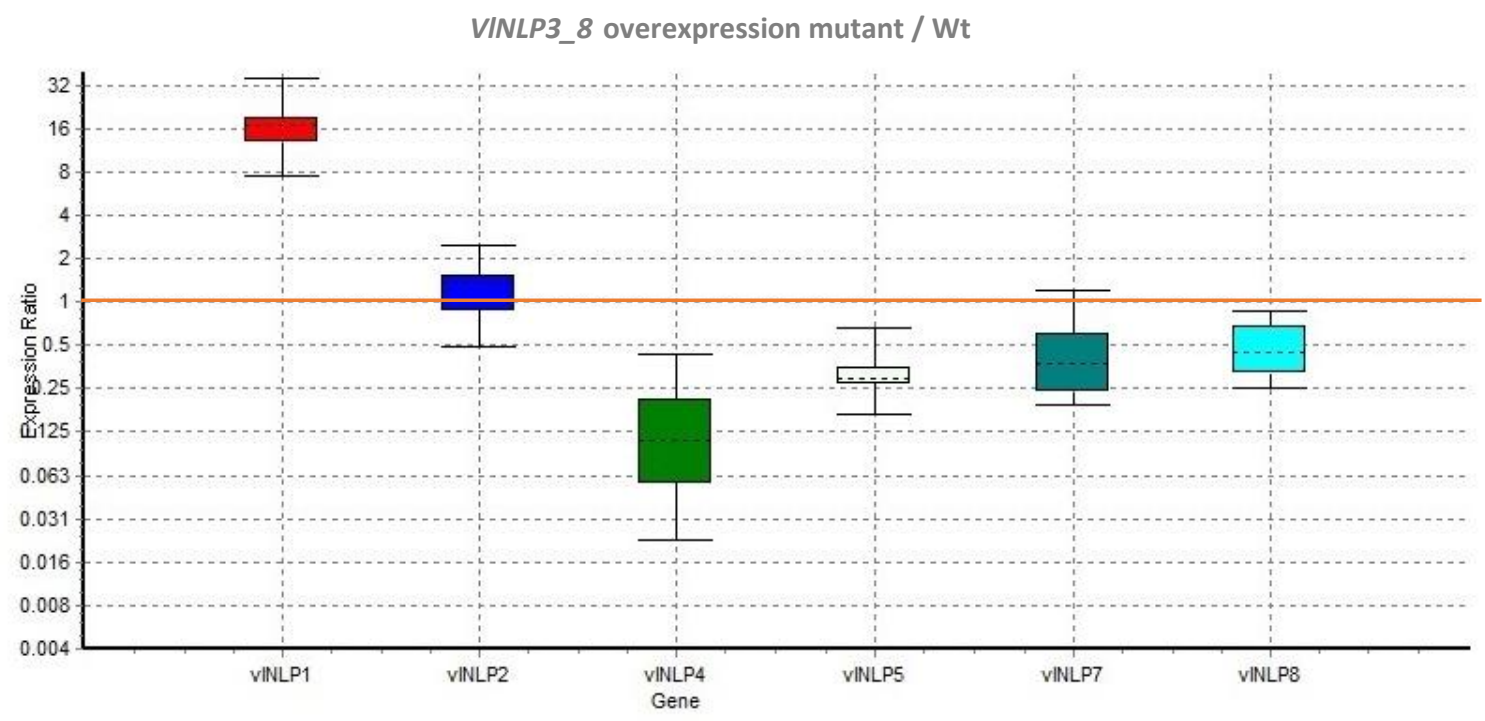

Fig. 19 Effect of the overexpression of VINLP3_8 on the expression of other VINLPs in VL43. The relative expression ratio figure with the modification using reference gene tubulin was created by software REST2009. Boxes are the $75^{\text {th }}$ percentile, median and the $25^{\text {th }}$ percentile. The box range above the orange line represents up-regulation, below down-regulation. Tests conducted in triplicate.

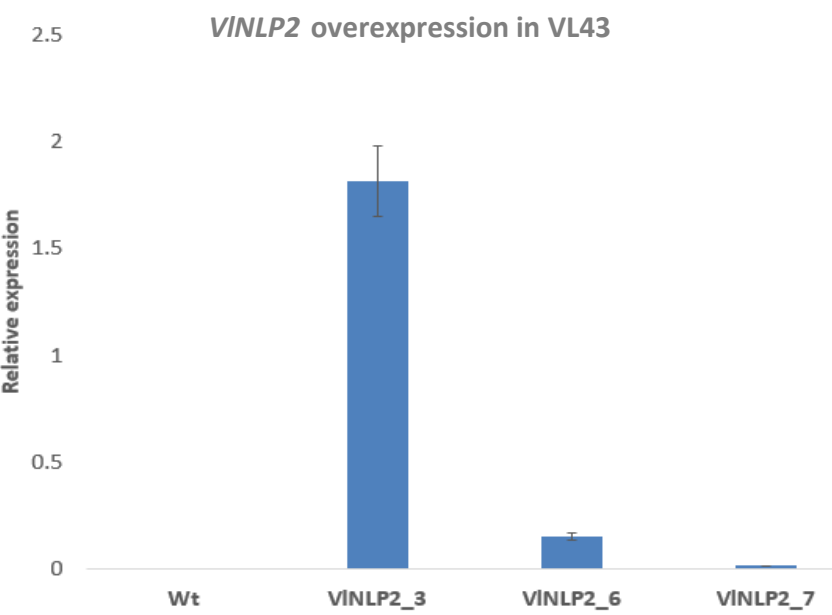

Fig. 20 Expression levels of $V I N L P 2$ in wild type and three VINLP2 overexpression mutants of $V$. longisporum VL43. The relative expression represents the transcript level with modification using reference gene $G A P D H$. Bars represent the mean of tests conducted in triplicate \pm standard error. 


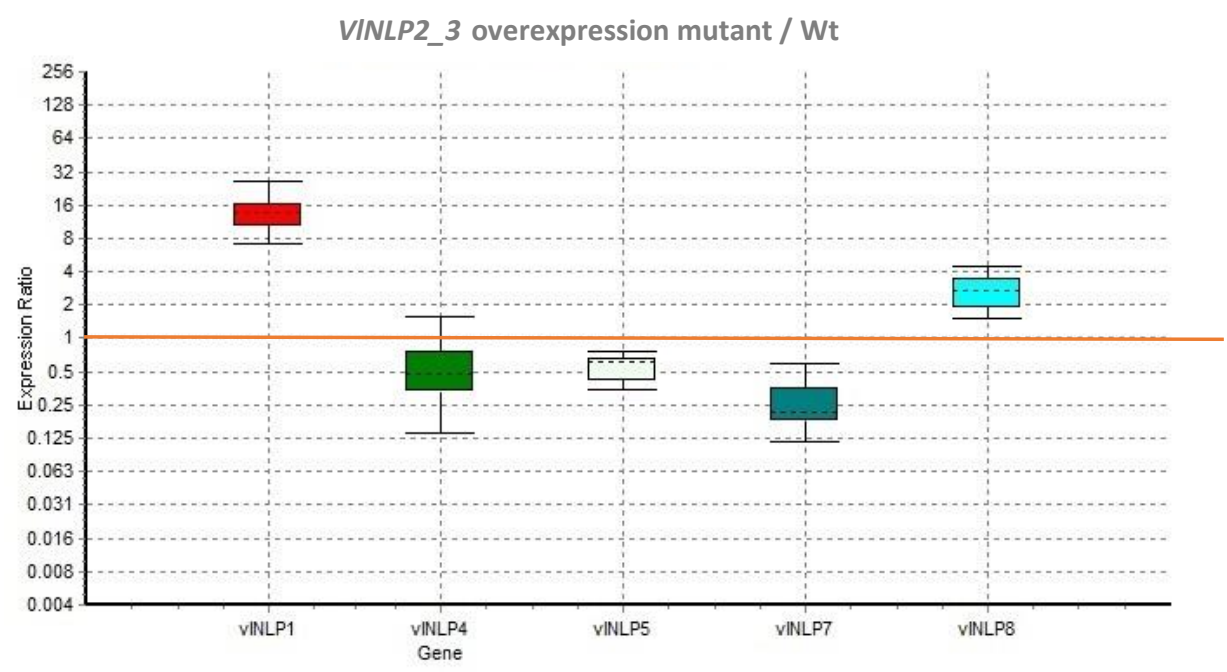

Fig. 21 Effect of the overexpression of VINLP2 on other VINLPS in VL43. The relative expression ratio figure with the modification using reference gene tubulin was created by software REST2009. Boxes are the $75^{\text {th }}$ percentile, median and the $25^{\text {th }}$ percentile. The box range above the orange line represents upregulation, below down-regulation. Tests conducted in triplicate.

\section{Plant infection experiments with mutants overexpressing VINLP2 and VINLP3}

Both VL43 mutant strains overexpressing VINLP3 and VINLP2 caused sever disease symptom and strong plant height reduction in oilseed rape, which were the same as wild type VL43 (Fig. 22 and 23). The fungal level of all tested wild type and mutants in hypocotyls of oilseed rape were also similar (Fig. 24). These results were confirmed in second independent experiment with identical treatments (Fig. 25, 26 and 27). The obtained results support the notion that expression of VINLP3 and VlNLP2 does not affect pathogenicity. 


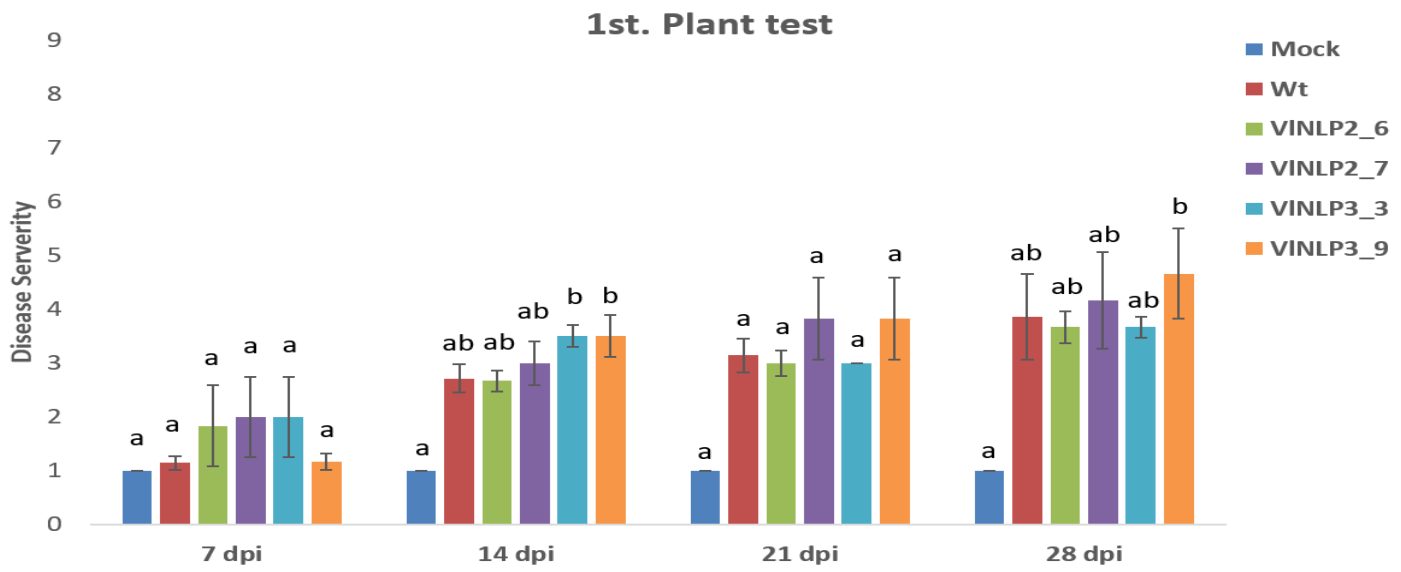

Fig. 22 Severity of diseases of oilseed rape infected with wild type VL43 and mutants overexpressing VINLP2 and VINLP3 in a climate chamber. Letters represent statistically significant differences (Kruskal-Wallis test, $\mathrm{p}<0.01)$. Six to seven plants were used infected with each isolate.

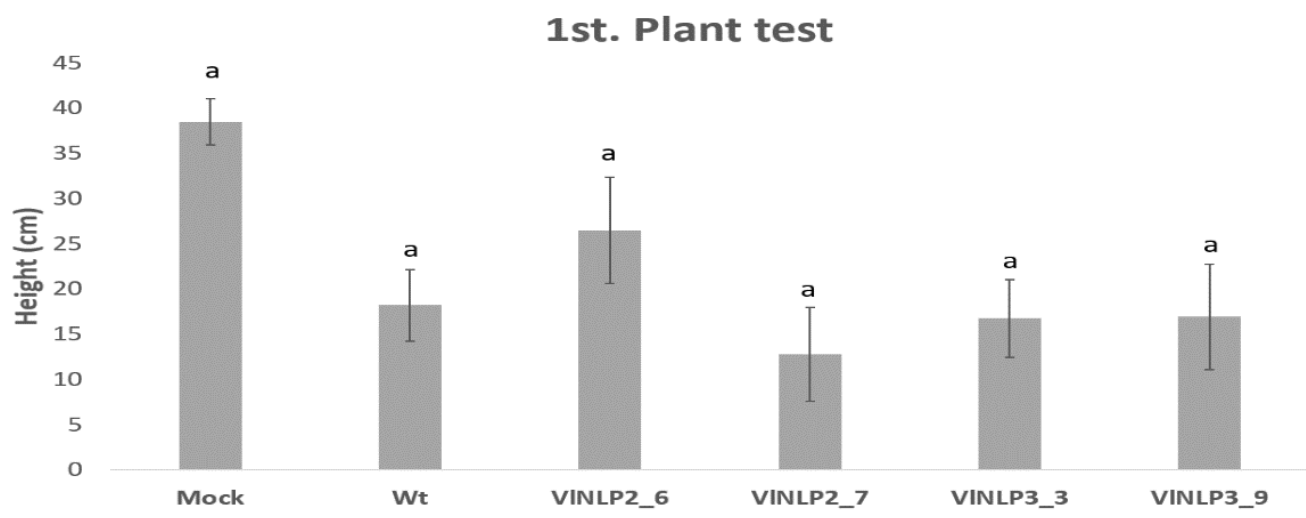

Fig. 23 Effect of the overexpression of VINLP2 and VINLP3 on the height of infected B. napus plants in a climate chamber. Letters represent statistically significant differences (Kruskal-Wallis test, $\mathrm{p}<0.01$ ). Six to seven plants were used infected with each isolate.

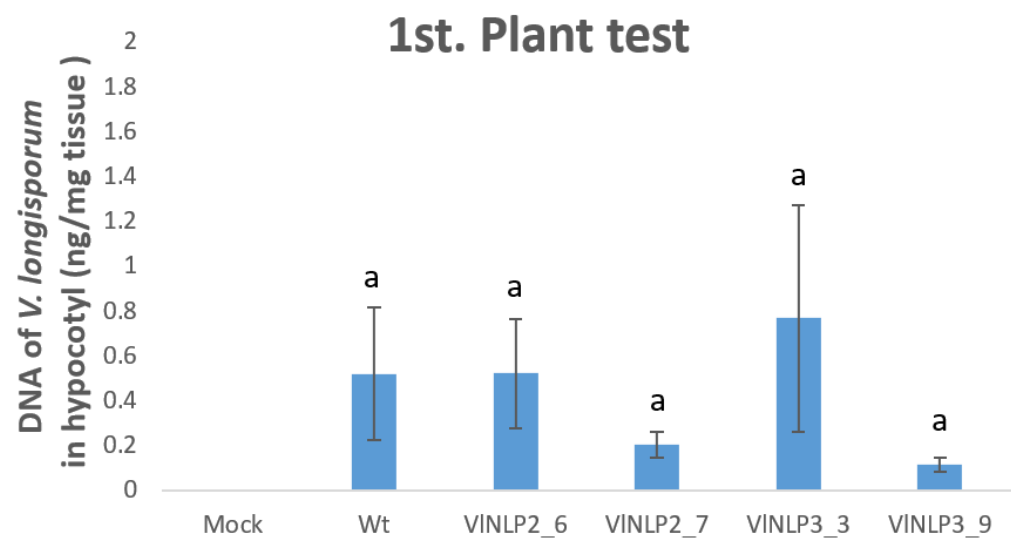

Fig. 24 Effect of the overexpression of VINLP2 and VINLP3 on fungal biomass in hypocotyl of infected $B$. napus plants in a climate chamber. Letters represent statistically significant differences (Kruskal-Wallis test, $\mathrm{p}<0.01$ ). Six to seven plants were used infected with each isolate. Mock plants were not included in statistic analysis. 


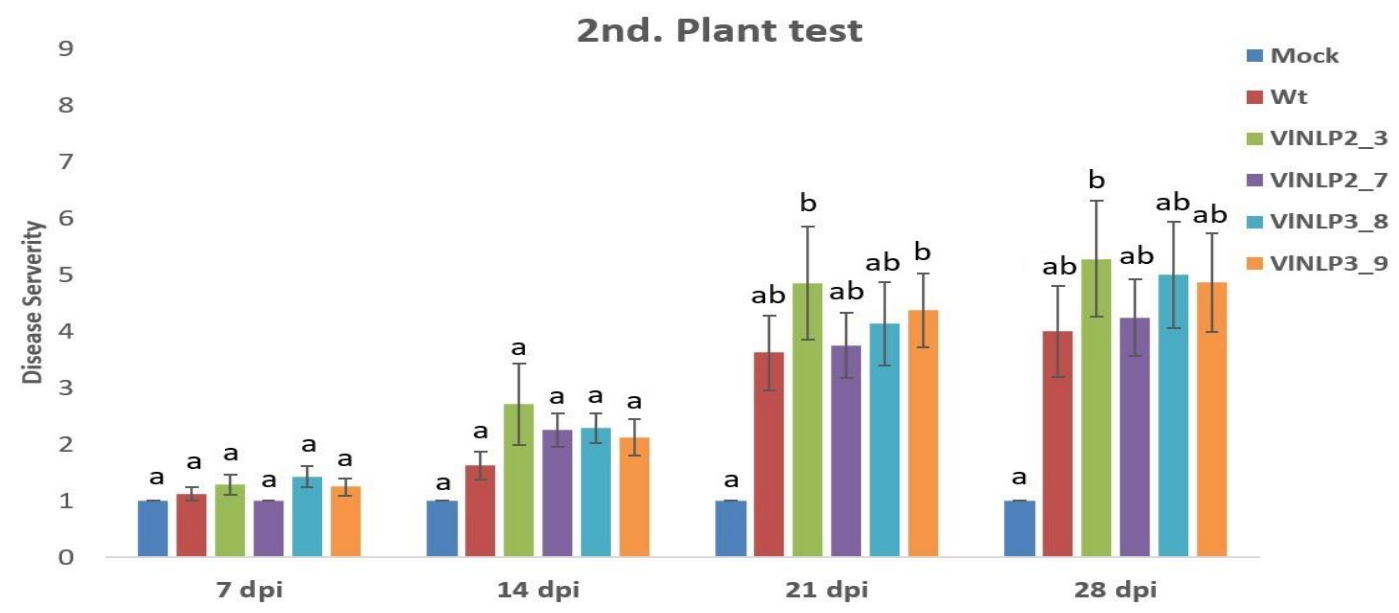

Fig. 25 Severity of diseases of oilseed rape infected with wild type VL43 and mutants overexpressing VINLP2 and VINLP3 in a green house. Letters represent statistically significant differences (Kruskal-Wallis test, $\mathrm{p}<0.01$ ). Six to seven plants were used infected with each isolate.

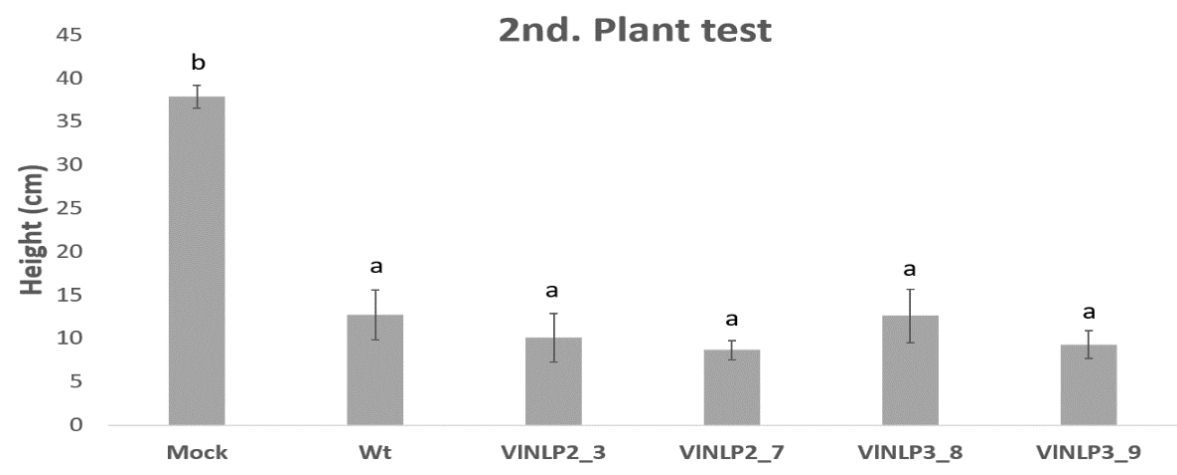

Fig. 26 Effect of the overexpression of $V I N L P 2$ and $V I N L P 3$ on the height of infected $B$. napus plants in a green house. Letters represent statistically significant differences (One-way ANOVA followed by Tukey's HSD test, $\mathrm{p}<0.01)$. Six to seven plants were used infected with each isolate.

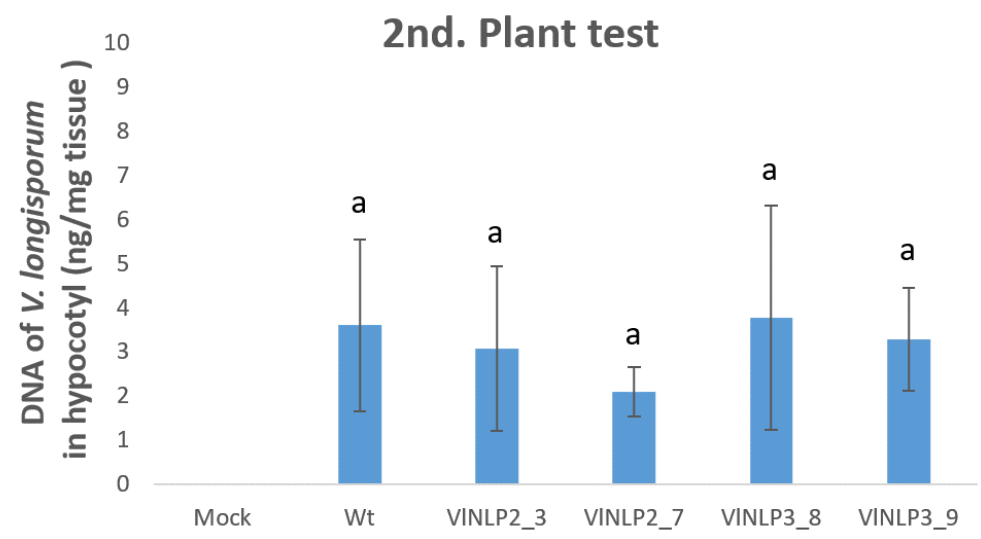

Fig. 27 Effect of the overexpression of VINLP2 and VINLP3 on fungal biomass in hypocotyl of infected $B$. napus plants in a green house. Letters represent statistically significant differences (Kruskal-Wallis test, $\mathrm{p}<0.01$ ). Six to seven plants were used infected with each isolate. Mock plants were not included in statistic analysis. 


\subsubsection{Do NLPs protect filamentous fungi from predation?}

\section{Predation failed to stimulate the production of NLP-proteins}

NLP-proteins are widely distributed among three kingdoms as described including microorganisms that are nonpathogenic to plants. Consequently, NLPs may serve for a more general purpose. Therefore, we hypothesized that the original biological role of NLPs is to defend soil microbes from predation.

Often, a specific threat triggers the expression or production of defense compounds . To explore, whether NLP-proteins expression can be induced by animal predation, two filamentous fungi, Verticillium longisporum and Fusarium graminearum, were subjected to nematode (Aphelenchoides saprophilus) or springtail grazing (Folsomia candida) and analyzed by RTqPCR for $V l N L P s$ and RNAseq for $F g N L P s$, respectively.

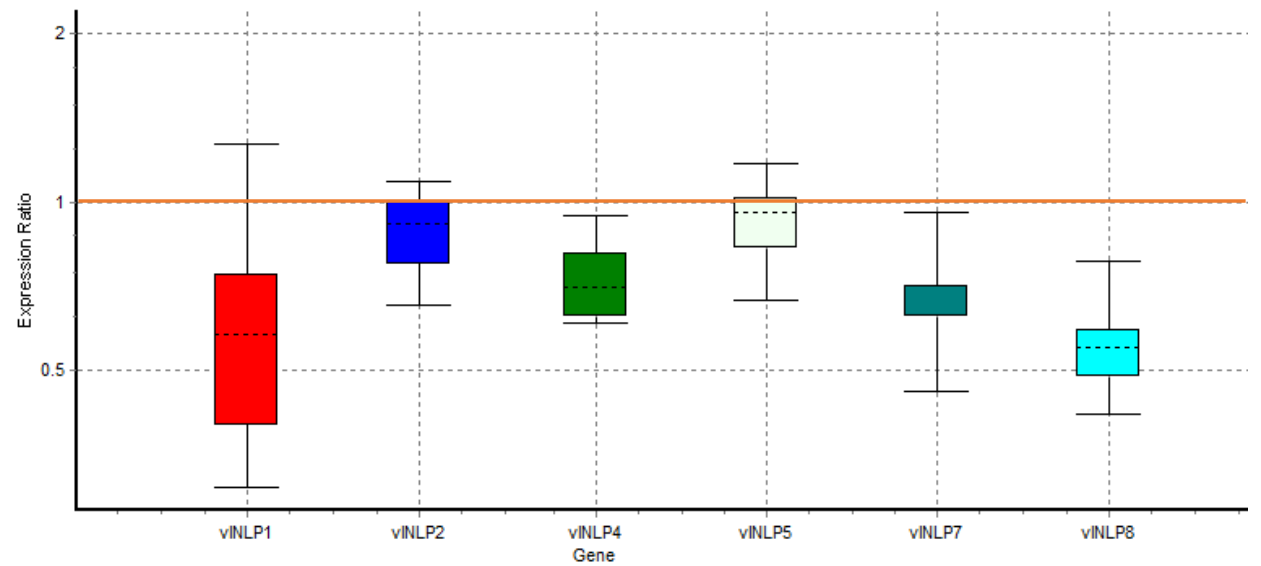

Fig. 28 Expression of VINLPS in $V$. longisporum exposed to feeding by the nematode Aphelenchoides saprophilus (RT-qPCR). RNA was extracted from $V$. longisporum mycelia exposed to nematodes $A$. saprophilus for 48 hours at $15^{\circ} \mathrm{C}$ in dark. The control fungi grew under the same condition. The relative expression ratio figure with the modification using reference gene tubulin was created by software REST2009. Boxes are the $75^{\text {th }}$ percentile, median and the $25^{\text {th }}$ percentile. The box range above the orange line represents up-regulation, below down-regulation. 
All VINLPs were not upregulated after $V$. longisporum was predated by nematodes. Four of VINLPs (VINLP1, VINLP4, VINLP7, VINLP8) were even slightly down-regulated (Fig. 28). Similar to $V$. longisporum, slight down regulation of FgNLPS was found in $F$. graminearum after exposure to grazing by $F$. candida for $48 \mathrm{~h}$ (Fig. 29). Expression of the three FgNLPs was checked by sequencing (RNAseq). FgNLPs information are listed in Suppl. Fig. 3. and Fig. 4.

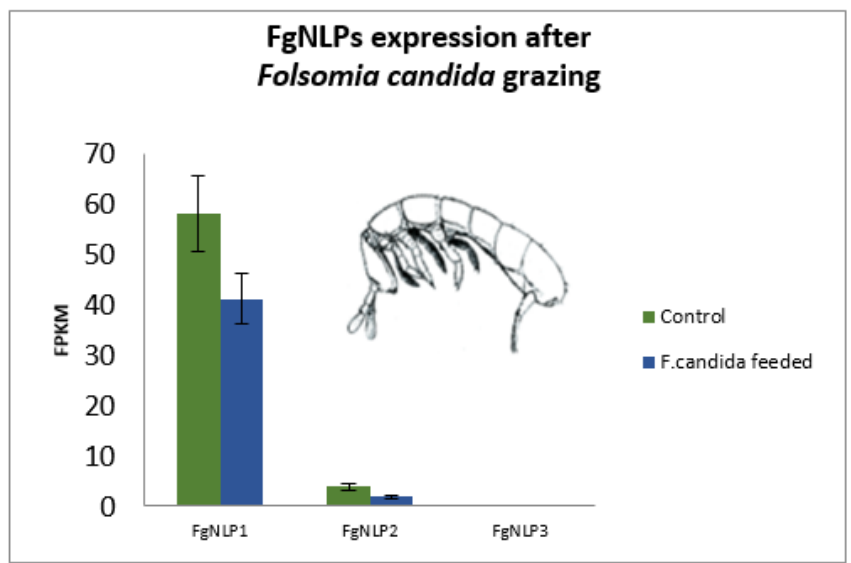

Fig. 29 Expression of FgNLPs in Fusarium graminearum exposed to grazing by the springtails Folsomia candida (RNAseq). RNA was extracted from $F$. graminearum mycelia exposed to $F$. candida for 48 hours at $15^{\circ} \mathrm{C}$ in dark. The control fungi grew under the same condition. Bars represent the mean of tests conducted in quadruplicates \pm standard error.

\section{NLP proteins do not affect food choice of predators}

F. candida food choice experiment showed random food preference between wild type and $M g N L P$-disrupted $Z$. tritici during time course assay.

Food choice experiments corroborated this conclusion. In the first experiment (Fig. 30), the $M g N L P$-disrupted mutant appeared to be preferred over the wild-type Z. tritici most of the time. However, repeated experiments showed different and often completely opposite preference. The experiments with the isopod $T$. tomentosa and the mealworm $T$. molitor showed similar results (data not shown). 


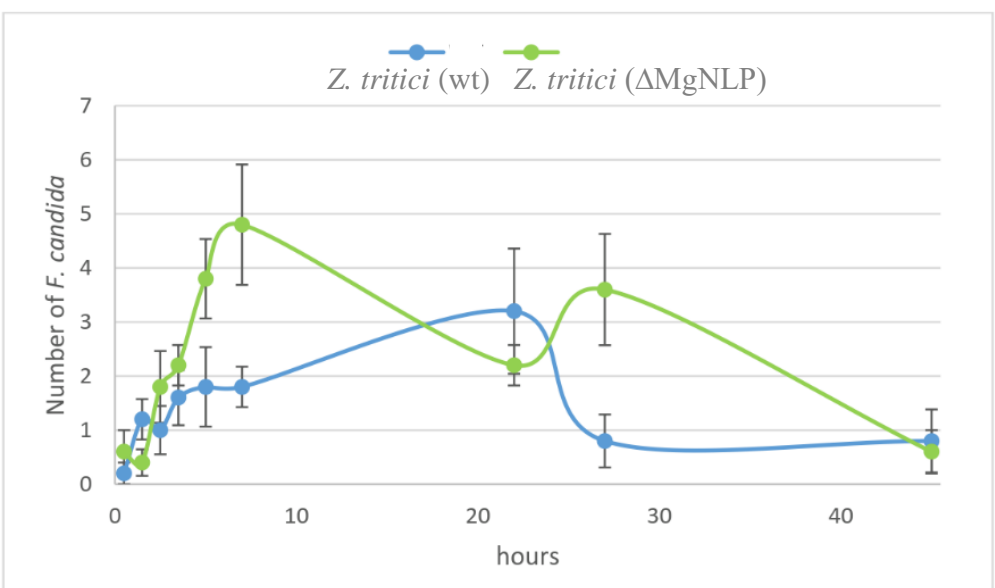

Fig. 30 Food preference of the springtail Folsomia candida for wild type and $M g N L P$-disrupted Zymoseptoria tritici. Twenty randomly selected 14-day-old $F$. candida were released in the center of the media. Afterwards, the counting were processed at $0.5,1.5,2.5,3.5,5,7,22,27$ and $45 \mathrm{~h}$ after their release on the plates. Points represent the mean of tests conducted with standard error.

\section{NLP proteins do not affect animal fitness}

F. candida growth were not affect by feeding on Z. tritici fungi with absence of $M g N L P$. Two indexes, animal body length and width, were used to represent fungal fitness in this study. Certain amount of animals with similar size were fed by either wild type Z. tritici or $\triangle M g N L P$ mutant, their body length and width were measured after 4 weeks incubation. As clearly shown in Fig. 31, there is no difference of body length and width between the animals which fed on $M g N L P$-containing $Z$. tritici or the $\triangle M g N L P$ mutant. Similar results were obtained with $T$. tomentosa and T. molitor as well (data not shown). The entire experiment was repeated once. The replicate gave us an identical result. NLP proteins cannot affect animal fitness. 


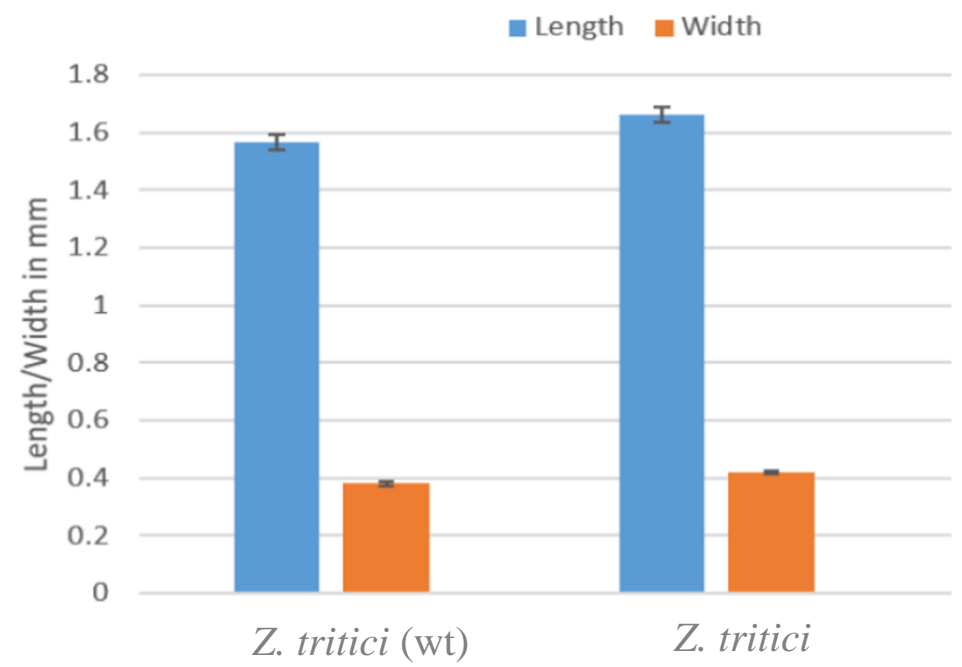

Fig. 31 Fitness of Folsomia candida fed on Zymoseptoria tritici and its $\triangle M g N L P$ mutant. Fifteen $F$. candida individuals were placed on fungal cultures and incubated in a dark room at $18^{\circ} \mathrm{C}$. After four weeks, the size of the killed $F$. candida was measured with a $\mathrm{mm}$ scale. Bars represent the mean of tests conducted with standard error. 


\subsection{Discussion}

\subsubsection{Comparison of NLPs from $V$. dahliae and $V$. longisporum}

The NLP-protein family is widespread among diverse microorganisms, including fungi, bacteria and oomycetes (Pemberton and Salmond 2004; Gijzen and Nürnberger 2006). Only ascomycete fungi harbor all three types of NLPs together, which suggests that their evolutionary origin might have occurred within them (Oome and van den Ackerveken 2014). In concurrence with a research of $V$. dahliae (Zhou et al. 2012), eight NLPs including all three types (VINLP1-8) occur in the ascomycete Verticillium longisporum . Its pathogen record and extensive NLP equipment turned this fungus into a suitable study object to explore the biological role of NLPS.

The close affinity between $V$. longisporum and $V$. dahliae reflects itself in the similarity of their NLPs (Zhou et al. 2012). The first VdNLP was identified from a cotton-pathogenic $V$. dahliae isolate VdLs.17 (Wang et al. 2004). Afterwards, ten additional VdNLPs were discovered in various $V$. dahliae isolates. Before the third type of $N L P S$ was defined, nine of them were thought to constitute NLPS. However, an alignment showed that VdNLP6 and VdNLP8 were less homologous to the others (Zhou et al. 2012). Due to its negative bit score, VdNLP8 is not a NLP member. Additionally, further research revealed that $V d N L P 8$ is not part of the $N L P$ family and VdNLP6 was absent in most of the investigated $V$. dahliae and other Verticillium spp., including V. albo-atrum and V. tricorpus (Santhanam et al. 2013). As type 3 NLPs was defined (Oome and van den Ackerveken 2014), VDAG_079720T0 turned also out to be a NLP family member, VdNLP10.

The genome of both $V$. longisporum isolates VL43 and VL32 did not contain a gene similar to VdNLP6 that could not be found in many $V$. dahliae isolates as well. Currently, the presence of VdNLP6 is only known for the VdLs17 and Vd592 isolates of V. dahliae. This provides evidence against the assumption that VdL43 and VdL32 represent hybrids of VdLs17 and Vd592.

Amino acid sequences of eight VINLPs show over 95 percent identity to VdNLPs, it indicates that the characteristics and function of VdNLPs are also applicable to VINLPs. Accordingly, VdNLPs were used for NLP-proteins comparison between $V$. dahliae and V. longisporum in this study. In order to maintain consistency in the present study, VINLPs were named based on Malte Beinhoff's thesis (Beinhoff 2011), which differ from VdNLPs name (Table 2). 
VINLPS show similarity with NLPs from other Verticillium species (in addition to VdNLPs) or even other microorganisms, suggesting strong conservation in the amino acid sequence of VINLPs and those in other microbe species. This might imply the existence of a functional constraint among pathogens that infect different plants. Conversely, NLPS are not likely to be involved in pathogen host specificity.

\subsubsection{Necrosis-inducing activity of NLPs}

Zhou et al. (2012) and Santhanam et al. (2013) studied the cytotoxic effects of VdNLPs and obtained concurring results. One study used the cotton-pathogen $V$. dahliae V592 and the other the lettuce-pathogen $V$. dahliae VdLs.17. Due to the remarkably high amino acid sequence similarity of NLPs between $V$. dahliae and $V$. longisporum, any potential cytotoxic activity of the eight VINLPs could be deduced by simple comparison. VdNLP1 (homologue to VINLP1) and VdNLP2 (homologue to VINLP6) were able to induce necrosis by Agrobacterium spp. infiltration and His-tagged purified protein infiltration in dicotyledonous plant leaves.

The necrosis formation requires conserved amino acid sequences. Any exchange of individual amino acids can cause activity loss. Figure 8 indicates the position of the conserved amino acid regions within VINLPs. Those that are required for necrosis formation are marked either in red or in blue. Therefore, VINLP1 and VINLP6 are deduced as cytotoxic NLPs.

However, VINLP6 was not identified properly in V. longisporum and attempts to amplify it from V. longisporum VL43 isolate failed. Therefore, only VINLP1 could be tested in this study and was found to cause necrotic lesions on plant leaves.

\subsubsection{Activation of plant immunity-associated responses by NLPs}

Böhm et al. (2014b) suggested that NLPs trigger immune responses in plants in two ways, plant PTI activated by cytotoxic PAMPs (or DAMPs) and ETI triggered by effector proteins from microbes. Some members of NLP protein family can cause necrotic effects and are capable of destroying the plant cell plasma membrane by causing leakages (Ottmann et al. 2009). The NLP phytotoxicity was identified among all three NLP-types .Therefore, plant immune responses could be activated by the releasing of immunogenic DAMPs after NLP-mediated plant cell damage (Ottmann et al. 2009). 
However, abolishment of necrosis activities of type 1 and type 2 NLPs showed completely opposite effects on triggering of plant immunity. Two necrotic NLPs, Pectobacterium carotovorum $p v$. carotovorum-derived $\operatorname{NLP}_{P c c}$ (type 2) and Phytophthora parasitica-derived $\mathrm{NLP}_{P p}$ (type 1), were involved. Heat denaturation or mutation of essential amino acid residues failed to affect the plant defense-eliciting activity of NLP $_{P p}$ but successfully destroyed this ability of $\operatorname{NLP}_{P c c}$ (Böhm et al. 2014b). This, the defense-stimulating activity of NLP Pcc $_{\text {may }}$ be only based on its necrotoxicity, while NLP $_{P p}$ triggers PTI in two ways by inflicting cell damage and another mode that is not linked to its necrosis-inducing activity. The second mode is ETI that is activated by a specific pattern of 20 amino acid pattern (nlp20). NLP ${ }_{P c c}$ lacks two of the four

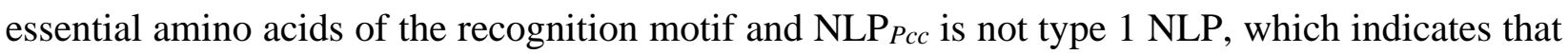
the four key amino acids and the number of potential disulfide bonds are two essential factors of ETI.

It is worth mentioning that the necrosis-inducing activities of NLPs can be entirely independent of ETI that is triggered by appropriate 20 amino acid motifs. Non-cytotoxic HaNLP proteins from H. arabidopsidis that harbor immunogenic motifs are able to activate defense responses without causing necrotic damage (Oome et al. 2014). The native HaNLP3 was an even slightly less efficient inducer of ethylene production (a fundamental plant immune response) than heat denatured HaNLP3, suggesting that the key residues may be located partially inside the NLP proteins.

MAPK activation test illustrated that the PTI in Arabidopsis was successfully triggered by the presence of V1NLP1. Genomic analysis revealed that nlp20(VINLP1) is also able to trigger pattern-induced plant immunity.

\subsubsection{Primary biological function of NLPs is unlikely related to the virulence of plant pathogens}

Bailey (1996) identified the Nep1 protein from the plant-pathogenic fungus Fusarium oxysporum. Therefore, the biological role of NLPs-family was originally considered to be related to fungal virulence on their host plants. However, their follow-up studies failed to confirm this hypothesis, because the disruption of NEP1 protein of $F$. oxysporum didn't affect its virulence on cocoa (Bailey et al. 2002). Since then, the role of the NLP protein family turned mysterious. 
In the following two decades, many research groups tried to verify the relationship between NLPs and pathogenicity towards their host plants. However, the diverse results of these studies did not allow clear conclusions. Table 9 summarizes the most important studies on effects that are caused by NLPS gene-disruption on pathogen virulence. It indicates that some members of NLP-family contribute to virulence on specific host plants, while some do not.

Firstly, the deletion of NLPS from some pathogens compromised their virulence, which was confirmed for fungi, bacteria and oomycetes. Disruption of the EccNip from the soft-rot bacteria Erwinia carotovora subsp. carotovora reduced its virulence on potato tubers, but not on potato stem (Mattinen et al. 2004). Three NLP-genes in Phytophthora capsici were shown to be required for full virulence of oomycetes (Feng et al. 2014). Reduction of Ssnep2 gene expression from Sclerotinia sclerotiorum resulted in decreased virulence on its host plant, Brassica napus (Dallal Bashi et al. 2010). Deletion of PeNLP1, but not PeNLP2, affected the virulence of post-harvest pathogen Penicillium expansum on apple fruits (Levin et al. 2019). Remarkably, disruption of either VdNLP1 (equal to VINLP1) or VdNLP2 (equal to VINLP6) genes encoding cytotoxic NLPs from Verticillium dahliae compromised its virulence on tomato and Arabidopsis, but only deletion of VdNLP1, not VdNLP2, affected its virulence on Nicotiana benthamiana (Santhanam et al. 2013). More interestingly, neither $V d N L P 1$ nor $V d N L P 2$ are essential to $V$. dahliae virulence on cotton plants. Even the simultaneous disruption of both genes failed to reduce virulence (Zhou et al. 2012). NLPs appear to have host-specific functions. It is noticeable, however, the reduced plant symptom representing less virulence may not always have biological meaningful in reality, for example, $15 \%$ less diameter of the lesion.

The virulence reduction was also observed in our previous study of VINLP. Beinhoff (2011) showed that disruption of VINLP1 resulted in a partial loss of virulence of VL43 on its host Brassica napus. B. napus plants infected by VINLPI knockout V. longisporum isolates were obviously higher than the plants infected by wild type fungi.

However, other studies provided opposite results. The wheat pathogen Mycosphaerella graminicola contains only a single $N L P$, but the $N L P$-deletion strain was equal to virulent (Motteram et al. 2009). Researches on two Botrytis fungi, Botrytis cinerea and Botrytis elliptica, showed that the disruption of NLPS genes failed to influence the virulence on their hosts tomato and lily (Cuesta Arenas et al. 2010; Staats et al. 2007). Even if the four MoNLPs in Magnaporthe oryzae had been disrupted together, virulence remained intact (Fang et al. 2017). 
Certain conditions are required for $N L P$ genes expression in some microorganisms. For example, Nep gene from the bacteria Erwinia carotovora was only expressed after the contact with solid medium (Mattinen et al. 2004). In contrast, except VdNLP5, most VdNLPs of the cotton-pathogen $V$. dahliae (V592) were expressed in fungal hyphae or spores without requiring specific conditions (Zhou et al. 2012). Our results indicate that VINLPs have a similar expression pattern as VdNLPs, which is that VlNLP3 (equal to VdNLP5) gene is also not expressed in the VL43 isolate when cultured in SXM.

Current researches studies mainly focus on the expressed NLPs, but ignore the fact that a variety of NLP genes are suppressed. For instance, 13 out of 33 NLP genes are suppressed in $P$. sojae (Dong et al. 2012), whereas 4 of 12 NLPs from H. arabidopsidis are also not expressed during infection (Cabral et al. 2012). More interestingly, our study shows that VlNLP3 suppression is strain-specific in VL43. Its expression was only detected in the isolates that belong to the A1/D1 and A1/D3 lineages including VL787, VL1194 and VL32. In addition to lineages difference, VINLP3 expression is possibly related to fungal virulence as well. The preliminary results indicated that avirulent isolates may have better capability to express $V I N L P 3$, which matches to our assumption that suppression of VINLP3 expression is a prerequisite for pathogenicity. Although VL1194 is an oilseed rape virulent strain, its VINLP3 transcript level is much lower than in the avirulent VL32 isolate from the same A1/D3 lineage as VL1194.

Unexpectedly, in the root-dipping experiment, the oilseed rape plants inoculated with VlNLP3 overexpressed VL43 spore suspension failed to show any disease severity reduction compared with plants infected by the wild type strain, neither disease symptoms nor fungal DNA amounts in plant hypocotyl tissue. According to these results, VINLP3 is not involved in pathogenicity.

In conclusion, the current insights of the role of NLP protein family are still controversial. The disruption of NLP genes failed to affect fungal virulence in many cases. Conclusively, the obtained data suggest that the original biological role of NLPs may not be related to plant pathogenicity. Besides, the study of VdNLPs from $V$. dahliae can also be involved in fungal vegetative growth and conidiospore production, but these results are only supported by a single published study (Santhanam et al. 2013). 


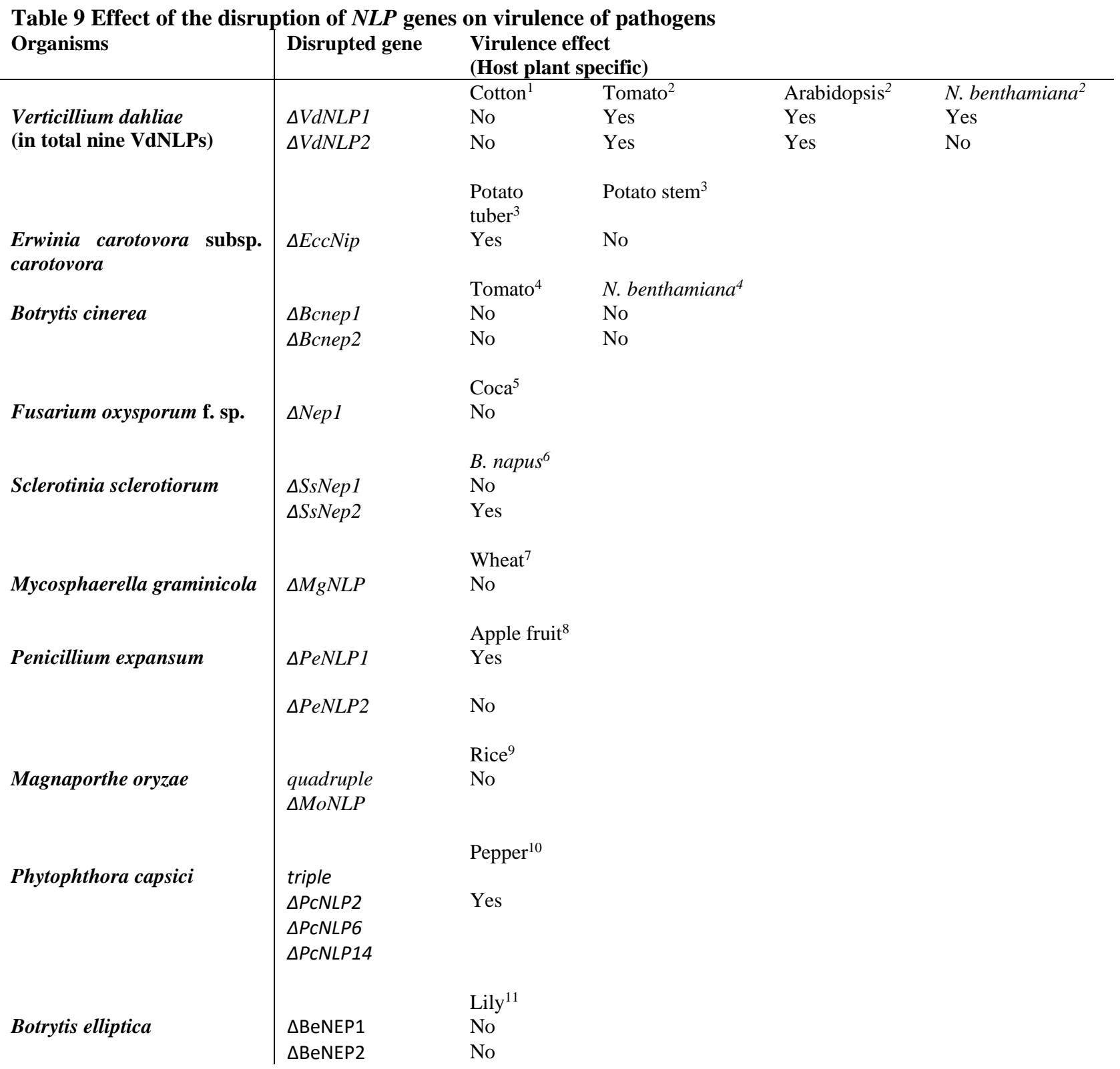

References: 1: Dou and Zhou (2012); 2:Santhanam et al. (2013); 3.Mattinen et al. (2004); 4.Cuesta Arenas et al. (2010); 5.Bailey et al. (2002); 6.Dallal Bashi et al. (2010); 7.Motteram et al. (2009); 8.Levin et al. (2019); 9.Fang et al. (2017); 10.Feng et al. (2014); 11. Staats et al. (2007)

\subsubsection{Biological function of NLPs in defense against predation was not supported}

NLPs proteins are widely distributed and identified from the microorganisms, many of which are not plant pathogens. Even in plant pathogens, in the last three decades, many studies proved that NLPs are not always involved in virulence. For this reason, we assume that the original role of NLP-family may be independent of plant pathogenicity, but be more general. 
Non-toxic compounds can also be involved in protecting fungi against a wide range of predators as described in Article III. The role as defense compounds matches our above stated assumption of a more general role. Therefore, we hypothesized that NLP-family act as defense compounds against fungal predators.

However, all experiments aimed at exploring this aspect failed to support our hypothesis. Normally, gene expression induction experiments indicate a potential function. Animal predation, however, failed to stimulate the expression of $N L P$ genes. Two filamentous ascomycetes fungi, $V$. longisporum and F. graminearum, were damaged by two different feeding modes: direct feeding by nematodes and grazing by springtails. None of them showed induction of NLPs compared with NLPs expression in undamaged fungal mycelia.

Besides, more direct results from food preference and animal fitness tests revealed that whether fungi contain NLPs does not affect fungal food choice and animal growth. Z. tritici possesses a single NLP protein. Therefore, its $\triangle M g N L P$ mutant is an ideal test organism for this purpose. No preference of three animals, F. candida, T. tomentosa and T. molitor, was detected for the mutant lacking the MgNLP protein. Also, the growth of tested three animals did not benefit from feeding on the $\triangle M g N L P$ mutant. In addition, the nematodes, Caenorhabditis elegans, did not show any food preference or better growth to transgenic E. coli strain which can produce VINLP1 protein (data not shown).

In conclusion, the role of NLP-family is still unclear. It is not related to fungal self-protection against predators but has nothing effect on plant pathogenicity as well. These results can be interpreted as an encouragement that research on NLPs biological role should not be restricted to plant disease alone.

\section{References}

Albert, Isabell; Böhm, Hannah; Albert, Markus; Feiler, Christina E.; Imkampe, Julia; Wallmeroth, Niklas et al. (2015): An RLP23-SOBIR1-BAK1 complex mediates NLP-triggered immunity. In Nature plants 1, p. 15140. DOI: 10.1038/nplants.2015.140.

Bailey, B. A.; Apel-Birkhold, Patricia C.; Luster, Douglas G. (2002): Expression of NEP1 by Fusarium oxysporum f. sp. erythroxyli After Gene Replacement and Overexpression Using Polyethylene Glycol-Mediated Transformation. In Phytopathology 92 (8), pp. 833-841. DOI: 10.1094/PHYTO.2002.92.8.833.

Bailey, Bryan A. (1996): Purification of a Protein from Culture Filtrates of Fusarium oxysporum that Induces Ethylene and Necrosis in Leaves of Erythroxylum coca. In Phytopathology 85 (10), p. 1250. DOI: 10.1094/Phyto85-1250. 
Beinhoff, Malte (2011): Molecular and functional characterization of potential pathogenicity related genes from Verticillium longisporum. PhD dissertation. Georg August University, Göttingen, Germany. Available online at http://hdl.handle.net/11858/00-1735-0000-0006-AB46-F.

Bendtsen, Jannick Dyrløv; Nielsen, Henrik; Heijne, Gunnar von; Brunak, Søren (2004): Improved prediction of signal peptides. SignalP 3.0. In Journal of molecular biology 340 (4), pp. 783-795. DOI: 10.1016/j.jmb.2004.05.028.

Berlanger, I. and Powelson, M.L. (2000): Verticillium wilt. Available online at http://www.apsnet.org/edcenter/intropp/lessons/fungi/ascomycetes/Pages/VerticilliumWilt.aspx.

Böhm, Hannah; Albert, Isabell; Fan, Li; Reinhard, André; Nürnberger, Thorsten (2014a): Immune receptor complexes at the plant cell surface. In Current opinion in plant biology 20, pp. 47-54. DOI:

10.1016/j.pbi.2014.04.007.

Böhm, Hannah; Albert, Isabell; Oome, Stan; Raaymakers, Tom M.; van den Ackerveken, Guido; Nürnberger, Thorsten (2014b): A conserved peptide pattern from a widespread microbial virulence factor triggers pattern-induced immunity in Arabidopsis. In PLoS pathogens 10 (11), e1004491. DOI: 10.1371/journal.ppat.1004491.

Brandfass, Christoph; Karlovsky, Petr (2008): Upscaled CTAB-based DNA extraction and real-time PCR assays for Fusarium culmorum and $F$. graminearum DNA in plant material with reduced sampling error. In International journal of molecular sciences 9 (11), pp. 2306-2321. DOI: 10.3390/ijms9112306.

Cabral, Adriana; Oome, Stan; Sander, Nick; Küfner, Isabell; Nürnberger, Thorsten; van den Ackerveken, Guido (2012): Nontoxic Nep1-like proteins of the downy mildew pathogen Hyaloperonospora arabidopsidis. Repression of necrosis-inducing activity by a surface-exposed region. In Molecular plant-microbe interactions : MPMI 25 (5), pp. 697-708. DOI: 10.1094/MPMI-10-11-0269.

Chen, Xiao-Ren; Huang, Shen-Xin; Zhang, Ye; Sheng, Gui-Lin; Li, Yan-Peng; Zhu, Feng (2018): Identification and functional analysis of the NLP-encoding genes from the phytopathogenic oomycete Phytophthora capsici. In Molecular genetics and genomics : MGG 293 (4), pp. 931-943. DOI: 10.1007/s00438-018-1432-7.

Clewes, E.; Edwards, S. G.; Barbara, D. J. (2008): Direct molecular evidence supports long-spored microsclerotial isolates of Verticillium from crucifers being interspecific hybrids. In Plant Pathology 57 (6), pp. 1047-1057. DOI: 10.1111/j.1365-3059.2008.01897.x.

Collins, A.; Mercado-Blanco, J.; Jimenez-Diaz, R. M.; Olivares, C.; Clewes, E.; Barbara, D. J. (2005): Correlation of molecular markers and biological properties in Verticillium dahliae and the possible origins of some isolates. In Plant Pathology 54 (4), pp. 549-557. DOI: 10.1111/j.1365-3059.2005.01240.x.

Collins, Alex; Okoli, C. Ada N.; Morton, Anne; Parry, David; Edwards, Simon G.; Barbara, Dez J. (2003): Isolates of Verticillium dahliae Pathogenic to Crucifers Are of at Least Three Distinct Molecular Types. In Phytopathology 93 (3), pp. 364-376. DOI: 10.1094/PHYTO.2003.93.3.364.

Crous, P. W.; Schoch, C. L.; Hyde, K. D.; Wood, A. R.; Gueidan, C.; Hoog, G. S. de; Groenewald, J. Z. (2009): Phylogenetic lineages in the Capnodiales. In Studies in mycology 64, 17-47S7. DOI: 10.3114/sim.2009.64.02.

Cuesta Arenas, Yaite; Kalkman, Eric R.I.C.; Schouten, Alexander; Dieho, Mirjam; Vredenbregt, Peter; Uwumukiza, Beatrice et al. (2010): Functional analysis and mode of action of phytotoxic Nep1-like proteins of Botrytis cinerea. In Physiological and Molecular Plant Pathology 74 (5-6), pp. 376-386. DOI: 10.1016/j.pmpp.2010.06.003.

Dallal Bashi, Zafer; Hegedus, Dwayne D.; Buchwaldt, Lone; Rimmer, S. Roger; Borhan, Mohammed H. (2010): Expression and regulation of Sclerotinia sclerotiorum necrosis and ethylene-inducing peptides (NEPs). In Molecular plant pathology 11 (1), pp. 43-53. DOI: 10.1111/j.1364-3703.2009.00571.x.

David, Helga; Ozçelik, Ilknur S.; Hofmann, Gerald; Nielsen, Jens (2008): Analysis of Aspergillus nidulans metabolism at the genome-scale. In BMC genomics 9, p. 163. DOI: 10.1186/1471-2164-9-163.

Dean, Ralph; van Kan, Jan A. L.; Pretorius, Zacharias A.; Hammond-Kosack, Kim E.; Di Pietro, Antonio; Spanu, Pietro D. et al. (2012): The Top 10 fungal pathogens in molecular plant pathology. In Molecular plant pathology 13 (4), pp. 414-430. DOI: 10.1111/j.1364-3703.2011.00783.x.

Depotter, Jasper R. L.; Deketelaere, Silke; Inderbitzin, Patrik; Tiedemann, Andreas Von; Höfte, Monica; Subbarao, Krishna V. et al. (2016): Verticillium longisporum, the invisible threat to oilseed rape and other brassicaceous plant hosts. In Molecular plant pathology 17 (7), pp. 1004-1016. DOI: 10.1111/mpp.12350. 
DIXON, G. R.; PEGG, G. F. (1972): Changes in Amino-acid Content of Tomato Xylem Sap Following Infection with Strains of Verticillium albo-atrum. In Annals of Botany 36 (1), pp. 147-154. DOI:

10.1093/oxfordjournals.aob.a084567.

Dong, Suomeng; Kong, Guanghui; Qutob, Dinah; Yu, Xiaoli; Tang, Junli; Kang, Jixiong et al. (2012): The NLP toxin family in Phytophthora sojae includes rapidly evolving groups that lack necrosis-inducing activity. In Molecular plant-microbe interactions : MPMI 25 (7), pp. 896-909. DOI: 10.1094/MPMI-01-12-0023-R.

Dou, Daolong; Zhou, Jian-Min (2012): Phytopathogen effectors subverting host immunity. Different foes, similar battleground. In Cell host \& microbe 12 (4), pp. 484-495. DOI: 10.1016/j.chom.2012.09.003.

Drobne, Damjana (1997): Terrestrial isopods-a good choice for toxicity testing of pollutants in the terrestrial environment. In Environ Toxicol Chem 16 (6), pp. 1159-1164. DOI: 10.1002/etc.5620160610.

Dunker, S.; Keunecke, H.; Steinbach, P.; Tiedemann, A. von (2008): Impact of Verticillium longisporum on Yield and Morphology of Winter Oilseed Rape (Brassica napus ) in Relation to Systemic Spread in the Plant. In $J$ Phytopathol 156 (11-12), pp. 698-707. DOI: 10.1111/j.1439-0434.2008.01429.x.

Eynck, C.; Koopmann, B.; Grunewaldt-Stoecker, G.; Karlovsky, P.; Tiedemann, A. von (2007): Differential interactions of Verticillium longisporum and V. dahliae with Brassica napus detected with molecular and histological techniques. In Eur J Plant Pathol 118 (3), pp. 259-274. DOI: 10.1007/s10658-007-9144-6.

Eynck, C.; Koopmann, B.; Tiedemann, A. von (2009): Identification of Brassica accessions with enhanced resistance to Verticillium longisporum under controlled and field conditions. In J Plant Dis Prot 116 (2), pp. 63-72. DOI: $10.1007 / \mathrm{BF} 03356288$.

Fang, Ya-Li; Peng, You-Liang; Fan, Jun (2017): The Nep1-like protein family of Magnaporthe oryzae is dispensable for the infection of rice plants. In Scientific reports 7 (1), p. 4372. DOI: 10.1038/s41598-017-04430-0.

Felix, Georg; Duran, Juliana D.; Volko, Sigrid; Boller, Thomas (1999): Plants have a sensitive perception system for the most conserved domain of bacterial flagellin. In The Plant Journal 18 (3), pp. 265-276. DOI: 10.1046/j.1365313X.1999.00265.x.

Fellbrich, Guido; Romanski, Annette; Varet, Anne; Blume, Beatrix; Brunner, Frédéric; Engelhardt, Stefan et al. (2002): NPP1, a Phytophthora-associated trigger of plant defense in parsley and Arabidopsis. In The Plant journal : for cell and molecular biology 32 (3), pp. 375-390.

Feng, Bao-Zhen; Zhu, Xiao-Ping; Fu, Li; Lv, Rong-Fei; Storey, Dylan; Tooley, Paul; Zhang, Xiu-Guo (2014): Characterization of necrosis-inducing NLP proteins in Phytophthora capsici. In BMC plant biology 14, p. 126. DOI: 10.1186/1471-2229-14-126.

Fradin, Emilie F.; Thomma, Bart P. H. J. (2006): Physiology and molecular aspects of Verticillium wilt diseases caused by V. dahliae and V. albo-atrum. In Molecular plant pathology 7 (2), pp. 71-86. DOI: 10.1111/j.13643703.2006.00323.x.

Garber, R. H. and Houston, B. R. (1966): Penetration and development of Verticillium albo-atrum in the cotton plant.

Gijzen, Mark; Nürnberger, Thorsten (2006): Nep1-like proteins from plant pathogens. Recruitment and diversification of the NPP1 domain across taxa. In Phytochemistry 67 (16), pp. 1800-1807. DOI: 10.1016/j.phytochem.2005.12.008.

Guo, Zhiqing; Pfohl, Katharina; Karlovsky, Petr; Dehne, Heinz-Wilhelm; Altincicek, Boran (2018): Dissemination of Fusarium proliferatum by mealworm beetle Tenebrio molitor. In PloS one 13 (9), e0204602. DOI: 10.1371/journal.pone.0204602.

Gust, Andrea A.; Felix, Georg (2014): Receptor like proteins associate with SOBIR1-type of adaptors to form bimolecular receptor kinases. In Current opinion in plant biology 21, pp. 104-111. DOI: 10.1016/j.pbi.2014.07.007.

Inderbitzin, Patrik; Bostock, Richard M.; Davis, R. Michael; Usami, Toshiyuki; Platt, Harold W.; Subbarao, Krishna V. (2011a): Phylogenetics and taxonomy of the fungal vascular wilt pathogen Verticillium, with the descriptions of five new species. In PloS one 6 (12), e28341. DOI: 10.1371/journal.pone.0028341.

Inderbitzin, Patrik; Davis, R. Michael; Bostock, Richard M.; Subbarao, Krishna V. (2011b): The ascomycete Verticillium longisporum is a hybrid and a plant pathogen with an expanded host range. In PloS one 6 (3), e18260. DOI: 10.1371/journal.pone.0018260. 
Inderbitzin, Patrik; Davis, R. Michael; Bostock, Richard M.; Subbarao, Krishna V. (2013): Identification and Differentiation of Verticillium Species and V. longisporum Lineages by Simplex and Multiplex PCR Assays. In PloS one 8 (6), e65990. DOI: 10.1371/journal.pone.0065990.

Inderbitzin, Patrik; Subbarao, Krishna V. (2014): Verticillium systematics and evolution. How confusion impedes Verticillium wilt management and how to resolve it. In Phytopathology 104 (6), pp. 564-574. DOI: 10.1094/PHYTO-11-13-0315-IA.

Janzen, Daniel H. (1977): Why Fruits Rot, Seeds Mold, and Meat Spoils. In The American Naturalist 111 (980), pp. 691-713. DOI: $10.1086 / 283200$.

Javidkar, Mohammad; Cooper, Steven J. B.; King, Rachael A.; Humphreys, William F.; Austin, Andrew D. (2015): Molecular phylogenetic analyses reveal a new southern hemisphere oniscidean family (Crustacea. Isopoda) with a unique water transport system. In Invert. Systematics 29 (6), p. 554. DOI: 10.1071/IS15010.

Kamble, A.; Koopmann, B.; Tiedemann, A. von (2013): Induced resistance to Verticillium longisporum in Brassica napus by $\beta$-aminobutyric acid. In Plant Pathology 62 (3), pp. 552-561. DOI: 10.1111/j.1365-3059.2012.02669.x.

Kanneganti, Thirumala-Devi; Huitema, Edgar; Cakir, Cahid; Kamoun, Sophien (2006): Synergistic interactions of the plant cell death pathways induced by Phytophthora infestans Nepl-like protein PiNPP1.1 and INF1 elicitin. In Molecular plant-microbe interactions : MPMI 19 (8), pp. 854-863. DOI: 10.1094/MPMI-19-0854.

Karapapa, V. K.; Bainbridge, B. W.; Heale, J. B. (1997): Morphological and molecular characterization of Verticillium longisporum comb, nov., pathogenic to oilseed rape. In Mycological Research 101 (11), pp. 1281-1294. DOI: $10.1017 /$ S0953756297003985.

Kleemann, Jochen; Rincon-Rivera, Linda J.; Takahara, Hiroyuki; Neumann, Ulla; van Loren Themaat, E. ver; van Themaat, Emiel Ver Loren et al. (2012): Sequential delivery of host-induced virulence effectors by appressoria and intracellular hyphae of the phytopathogen Colletotrichum higginsianum. In PLoS pathogens 8 (4), e1002643. DOI: 10.1371/journal.ppat.1002643.

Kumar, Sudhir; Stecher, Glen; Li, Michael; Knyaz, Christina; Tamura, Koichiro (2018): MEGA X. Molecular Evolutionary Genetics Analysis across Computing Platforms. In Molecular biology and evolution 35 (6), pp. 15471549. DOI: $10.1093 / \mathrm{molbev} / \mathrm{msy} 096$.

Leino, M. (2006): Fungal Diseases on Oilseed Rape and Turnip Rape.

Lenarčič, Tea; Albert, Isabell; Böhm, Hannah; Hodnik, Vesna; Pirc, Katja; Zavec, Apolonija B. et al. (2017): Eudicot plant-specific sphingolipids determine host selectivity of microbial NLP cytolysins. In Science (New York, N.Y.) 358 (6369), pp. 1431-1434. DOI: 10.1126/science.aan6874.

Levin, Elena; Raphael, Ginat; Ma, Jing; Ballester, Ana-Rosa; Feygenberg, Oleg; Norelli, John et al. (2019): Identification and Functional Analysis of NLP-Encoding Genes from the Postharvest Pathogen Penicillium expansum. In Microorganisms 7 (6). DOI: 10.3390/microorganisms7060175.

Lima, Juliana O.; Pereira, Jorge F.; Rincones, Johana; Barau, Joan G.; Araújo, Elza F.; Pereira, Gonçalo A. G.; Queiroz, Marisa V. (2009): The glyceraldehyde-3-phosphate dehydrogenase gene of Moniliophthoraperniciosa, the causal agent of witches' broom disease of Theobroma cacao. In Genetics and molecular biology 32 (2), pp. 362-366. DOI: $10.1590 / \mathrm{S} 1415-47572009000200024$

Macho, Alberto P.; Zipfel, Cyril (2014): Plant PRRs and the activation of innate immune signaling. In Molecular cell 54 (2), pp. 263-272. DOI: 10.1016/j.molcel.2014.03.028.

Majka, Christopher G.; Bouchard, Patrice; Bousquet, Yves (2008): Tenebrionidae (Coleoptera) of the Maritime Provinces of Canada. In Can Entomol 140 (6), pp. 690-713. DOI: 10.4039/n08-047.

Mattinen, Laura; Tshuikina, Marina; Mäe, Andres; Pirhonen, Minna (2004): Identification and characterization of Nip, necrosis-inducing virulence protein of Erwinia carotovora subsp. carotovora. In Molecular plant-microbe interactions : MPMI 17 (12), pp. 1366-1375. DOI: 10.1094/MPMI.2004.17.12.1366.

Motteram, Juliet; Küfner, Isabell; Deller, Siân; Brunner, Frédéric; Hammond-Kosack, Kim E.; Nürnberger, Thorsten; Rudd, Jason J. (2009): Molecular characterization and functional analysis of MgNLP, the sole NPP1 domaincontaining protein, from the fungal wheat leaf pathogen Mycosphaerella graminicola. In Molecular plant-microbe interactions : MPMI 22 (7), pp. 790-799. DOI: 10.1094/MPMI-22-7-0790. 
Nitta, Yukino; Ding, Pingtao; Zhang, Yuelin (2014): Identification of additional MAP kinases activated upon PAMP treatment. In Plant signaling \& behavior 9 (11), e976155. DOI: 10.4161/15592324.2014.976155.

Novakazi, Fluturë; Inderbitzin, Patrik; Sandoya, German; Hayes, Ryan J.; Tiedemann, Andreas Von; Subbarao, Krishna V. (2015): The Three Lineages of the Diploid Hybrid Verticillium longisporum Differ in Virulence and Pathogenicity. In Phytopathology 105 (5), pp. 662-673. DOI: 10.1094/PHYTO-10-14-0265-R.

Oome, Stan; Raaymakers, Tom M.; Cabral, Adriana; Samwel, Simon; Böhm, Hannah; Albert, Isabell et al. (2014): Nep1-like proteins from three kingdoms of life act as a microbe-associated molecular pattern in Arabidopsis. In Proceedings of the National Academy of Sciences of the United States of America 111 (47), pp. 16955-16960. DOI: 10.1073/pnas.1410031111.

Oome, Stan; van den Ackerveken, Guido (2014): Comparative and functional analysis of the widely occurring family of Nep1-like proteins. In Molecular plant-microbe interactions : MPMI 27 (10), pp. 1081-1094. DOI: 10.1094/MPMI-04-14-0118-R.

Ottmann, Christian; Luberacki, Borries; Küfner, Isabell; Koch, Wolfgang; Brunner, Frédéric; Weyand, Michael et al. (2009): A common toxin fold mediates microbial attack and plant defense. In Proceedings of the National Academy of Sciences of the United States of America 106 (25), pp. 10359-10364. DOI: 10.1073/pnas.0902362106.

Pedras, M. S.; Okanga, F. I. (1999): Strategies of cruciferous pathogenic fungi. Detoxification of the phytoalexin cyclobrassinin by mimicry. In Journal of agricultural and food chemistry 47 (3), pp. 1196-1202. DOI: 10.1021/jf980854r.

Pedras, M. S. C.; Minic, Zoran; Jha, Mukund (2008): Brassinin oxidase, a fungal detoxifying enzyme to overcome a plant defense -- purification, characterization and inhibition. In The FEBS journal 275 (14), pp. 3691-3705. DOI: 10.1111/j.1742-4658.2008.06513.x.

Pedras, M. Soledade C. (1998): A convenient synthesis of the cruciferous phytoalexins brassicanal A and brassilexin by mimicry of a fungal detoxification pathway. In Chem. Commun. (15), pp. 1565-1566. DOI: 10.1039/a803485k.

Pedras, M. Soledade C.; Abdoli, Abbas; Chumala, Paulos B.; Saha, Pijus; Schatte, Gabriele (2013): Unprecedented spirocyclization of 3-methyleneindoline-2-thiones during hydrolysis of the phytoalexin cyclobrassinin. In Bioorganic \& medicinal chemistry letters 23 (2), pp. 484-487. DOI: 10.1016/j.bmcl.2012.11.042.

Pedras, M. Soledade C.; Ahiahonu, Pearson W. K.; Hossain, Mohammad (2004): Detoxification of the cruciferous phytoalexin brassinin in Sclerotinia sclerotiorum requires an inducible glucosyltransferase. In Phytochemistry 65 (19), pp. 2685-2694. DOI: 10.1016/j.phytochem.2004.08.033.

Pedras, M. Soledade C.; Chumala, Paulos B.; Jin, Wei; Islam, Mohammed S.; Hauck, Dominic W. (2009): The phytopathogenic fungus Alternaria brassicicola. Phytotoxin production and phytoalexin elicitation. In Phytochemistry 70 (3), pp. 394-402. DOI: 10.1016/j.phytochem.2009.01.005.

Pedras, M. Soledade C.; Hossain, Mohammad (2006): Metabolism of crucifer phytoalexins in Sclerotinia sclerotiorum. Detoxification of strongly antifungal compounds involves glucosylation. In Organic \& biomolecular chemistry 4 (13), pp. 2581-2590. DOI: 10.1039/b604400j.

Pedras, M. Soledade C.; Hossain, Sajjad; Snitynsky, Ryan B. (2011): Detoxification of cruciferous phytoalexins in Botrytis cinerea. Spontaneous dimerization of a camalexin metabolite. In Phytochemistry 72 (2-3), pp. 199-206. DOI: 10.1016/j.phytochem.2010.11.018.

Pedras, M. Soledade C.; Suchy, Mojmir (2005): Detoxification pathways of the phytoalexins brassilexin and sinalexin in Leptosphaeria maculans. Isolation and synthesis of the elusive intermediate 3-formylindolyl-2-sulfonic acid. In Organic \& biomolecular chemistry 3 (10), pp. 2002-2007. DOI: 10.1039/b501907a.

Pegg, G.F; Brady, B. L. (2002): Verticillium wilts. Available online at https://doi.org/10.1079/9780851995298.0000.

Pemberton, Clare L.; Salmond, George P. C. (2004): The Nep1-like proteins-a growing family of microbial elicitors of plant necrosis. In Molecular plant pathology 5 (4), pp. 353-359. DOI: 10.1111/j.1364-3703.2004.00235.x.

Perez-Nadales, Elena; Nogueira, Maria Filomena Almeida; Baldin, Clara; Castanheira, Sónia; El Ghalid, Mennat; Grund, Elisabeth et al. (2014): Fungal model systems and the elucidation of pathogenicity determinants. In Fungal genetics and biology : FG \& B 70, pp. 42-67. DOI: 10.1016/j.fgb.2014.06.011. 
Pfaffl, M. W. (2001): A new mathematical model for relative quantification in real-time RT-PCR. In Nucleic acids research 29 (9), e45. DOI: 10.1093/nar/29.9.e45.

Ponge, J.-F. (2000): Vertical distribution of Collembola (Hexapoda) and their food resources in organic horizons of beech forests. In Biology and Fertility of Soils 32 (6), pp. 508-522. DOI: 10.1007/s003740000285.

Quaedvlieg, W.; Kema, G. H. J.; Groenewald, J. Z.; Verkley, G. J. M.; Seifbarghi, S.; Razavi, M. et al. (2011): Zymoseptoria gen. nov. A new genus to accommodate Septoria-like species occurring on graminicolous hosts. In Persoonia 26, pp. 57-69. DOI: 10.3767/003158511X571841.

Qutob, Dinah; Huitema, Edgar; Gijzen, Mark; Kamoun, Sophien (2003): Variation in structure and activity among elicitins from Phytophthora sojae. In Molecular plant pathology 4 (2), pp. 119-124. DOI: 10.1046/j.13643703.2003.00158.x.

Qutob, Dinah; Kemmerling, Birgit; Brunner, Frédéric; Küfner, Isabell; Engelhardt, Stefan; Gust, Andrea A. et al. (2006): Phytotoxicity and innate immune responses induced by Nep1-like proteins. In The Plant cell 18 (12), pp. 3721-3744. DOI: 10.1105/tpc.106.044180.

Rigden, Daniel J.; Galperin, Michael Y. (2004): The DxDxDG motif for calcium binding. Multiple structural contexts and implications for evolution. In Journal of molecular biology 343 (4), pp. 971-984. DOI: 10.1016/j.jmb.2004.08.077.

Santhanam, Parthasarathy; van Esse, H. Peter; Albert, Isabell; Faino, Luigi; Nürnberger, Thorsten; Thomma, Bart P. H. J. (2013): Evidence for functional diversification within a fungal NEP1-like protein family. In Molecular plantmicrobe interactions : MPMI 26 (3), pp. 278-286. DOI: 10.1094/MPMI-09-12-0222-R.

Santos, Eidy de O.; Alves, Nelson; Dias, Graciela M.; Mazotto, Ana Maria; Vermelho, Alane; Vora, Gary J. et al. (2011): Genomic and proteomic analyses of the coral pathogen Vibrio coralliilyticus reveal a diverse virulence repertoire. In The ISME journal 5 (9), pp. 1471-1483. DOI: 10.1038/ismej.2011.19.

Schmoll, Monika; Dattenböck, Christoph; Carreras-Villaseñor, Nohemí; Mendoza-Mendoza, Artemio; Tisch, Doris; Alemán, Mario Ivan et al. (2016): The Genomes of Three Uneven Siblings. Footprints of the Lifestyles of Three Trichoderma Species. In Microbiology and molecular biology reviews : MMBR 80 (1), pp. 205-327. DOI: 10.1128/MMBR.00040-15.

Sievers, Fabian; Wilm, Andreas; Dineen, David; Gibson, Toby J.; Karplus, Kevin; Li, Weizhong et al. (2011): Fast, scalable generation of high-quality protein multiple sequence alignments using Clustal Omega. In Molecular systems biology 7, p. 539. DOI: 10.1038/msb.2011.75.

Soledade, M.; Pedras, C.; Taylor, Janet L. (1993): Metabolism of the Phytoalexin Brassinin by the "Blackleg" Fungus. In J. Nat. Prod. 56 (5), pp. 731-738. DOI: 10.1021/np50095a010.

Staats, Martijn; van Baarlen, Peter; Schouten, Alexander; van Kan, Jan A. L. (2007): Functional analysis of NLP genes from Botrytis elliptica. In Molecular plant pathology 8 (2), pp. 209-214. DOI: 10.1111/j.13643703.2007.00382.x.

Stark, C. (1961): Das Auftreten der Verticillium: Tracheomykosen in Hamburger Gartenbaukulturen. (26:493-528).

Steventon, Lisa A.; Fahleson, Jan; Hu, Qiong; Dixelius, Christina (2002): Identification of the causal agent of Verticillium wilt of winter oilseed rape in Sweden, V. longisporum. In Mycological Research 106 (5), pp. 570-578. DOI: $10.1017 /$ S0953756202005865.

Thomma, Bart P. H. J.; Nürnberger, Thorsten; Joosten, Matthieu H. A. J. (2011): Of PAMPs and effectors. The blurred PTI-ETI dichotomy. In The Plant cell 23 (1), pp. 4-15. DOI: 10.1105/tpc.110.082602.

Tsuda, Kenichi; Katagiri, Fumiaki (2010): Comparing signaling mechanisms engaged in pattern-triggered and effector-triggered immunity. In Current opinion in plant biology 13 (4), pp. 459-465. DOI: 10.1016/j.pbi.2010.04.006.

Utermark, Jan; Karlovsky, Petr (2007): Role of zearalenone lactonase in protection of Gliocladium roseum from fungitoxic effects of the mycotoxin zearalenone. In Applied and environmental microbiology 73 (2), pp. 637-642. DOI: 10.1128/AEM.01440-06.

van den Ackerveken, Guido (2017): How plants differ in toxin-sensitivity. In Science (New York, N.Y.) 358 (6369), pp. 1383-1384. DOI: 10.1126/science.aar4188. 
Vega, Fernando E.; Goettel, Mark S.; Blackwell, Meredith; Chandler, David; Jackson, Mark A.; Keller, Siegfried et al. (2009): Fungal entomopathogens. New insights on their ecology. In Fungal Ecology 2 (4), pp. 149-159. DOI: 10.1016/j.funeco.2009.05.001.

Veit, Stefan; Wörle, Jörg Manfred; Nürnberger, Thorsten; Koch, Wolfgang; Seitz, Hanns Ulrich (2001): A Novel Protein Elicitor (PaNie) from Pythium aphanidermatum Induces Multiple Defense Responses in Carrot, Arabidopsis, and Tobacco. In Plant Physiol. 127 (3), pp. 832-841. DOI: 10.1104/pp.010350.

Wang, Jian-Ying; Cai, Yu; Gou, Jin-Ying; Mao, Ying-Bo; Xu, Yan-Hua; Jiang, Wei-Hong; Chen, Xiao-Ya (2004): VdNEP, an elicitor from Verticillium dahliae, induces cotton plant wilting. In Applied and environmental microbiology 70 (8), pp. 4989-4995. DOI: 10.1128/AEM.70.8.4989-4995.2004.

ZEISE, K.; Tiedemann, A. von (2002): Host Specialization among Vegetative Compatibility Groups of Verticillium dahliae in Relation to Verticillium longisporum. In J Phytopathol 150 (3), pp. 112-119. DOI: 10.1046/j.14390434.2002.00730.x.

Zhou, Bang-Jun; Jia, Pei-Song; Gao, Feng; Guo, Hui-Shan (2012): Molecular characterization and functional analysis of a necrosis- and ethylene-inducing, protein-encoding gene family from Verticillium dahliae. In Molecular plant-microbe interactions : MPMI 25 (7), pp. 964-975. DOI: 10.1094/MPMI-12-11-0319. 


\section{Supplement}

\section{Table 1 VINLPs sequences from various sources}

\begin{tabular}{|c|}
\hline \\
\hline $\begin{array}{l}\text { >VL43_VlNLP1_2018 } \\
\text { atgCTTCCCTCCACAATCTTCTCGGTCTTTGCCCTCGTCGGCAGCGCCTTGGCTCAGCATCCCCCCAAGGTTAACCACGACAGTATCA } \\
\text { ACCCCGTCCTCCACCCTCTGGGGCCCAACGGCGACATGATCAGGAAGTTCCAGCCTCTGCTTCACATTGCCCACGGTTGCCAGCCT } \\
\text { TACTCCGCTGTCAACACCCGCGGTGAGGTCAAGTAAGACACGCAGCACTCCCTTCAGCTTACACAAAGCTCCACGCTAACACATAT } \\
\text { TCTGCAGCGCCGGTCTCCAAGACAGCGGTACCACCGCAGGCGGCTGCAAGGAAACCAGCAAGGGCCAGACCTACGCCCGCTCCAT } \\
\text { GACCCTGAACGGCCAGTTCGGCATCATGTACGCCTGGTACTGGCCCAAGGACCAGCCCGCCGACGGCAACCTCGCCAGCGGCCAC } \\
\text { CGCCACGACTGGGAGAACGTCGTCATCTGGTTCAACTCGAACAACGCAAACCAGGCCGGCATCCTGCGCGGCGCCGCCTCGGGCC } \\
\text { ACGGCGACTACAAGAAGGTCAACAACCCCCAGCGCAACAACAACAACCTCCACGTCGAGTACTTCACCAGCCTCGGCAAGAACCA } \\
\text { CGAGCTGCAGTTCAAGACGTCGCCCGGCCGCACCTACTGGATCTGGGACTGGGACAGGATGGACAGCACCGTCCAGGGCGCCCTC } \\
\text { AACCGCGCCGACTTTGGCAGCGCCAACTGCCCCTTCAACAACAACAACTTTGAGAGGAACATGCGCGCCGCGTTTtaa }\end{array}$ \\
\hline $\begin{array}{l}\text { >VL32_VlNLP1_2018 } \\
\text { atgCTTCCCTCCACAATCTTCTCGGTCTTCGCCCTCGTCGGCAGCGCCTTGGCTCAGCAGCCTCCCAAGGTTAACCACGACAGCATCA } \\
\text { ACCCCATCCGCGATACTCTGGGACCCAACGGCAACATGATCAGGAAGTTCCAGCCTCTGCTTCACATTGCCCACGGTTGCCAGCCT } \\
\text { TACTCCGCCGTCAACACCCGCGGTGAGGTCAAGTAAGACACGCAACACACACTTCAGCTTCCACAAAACTCCTCGCTAACACATGC } \\
\text { TCTGCAGCGCCGGTCTCCAGGACAGCGGTACTACCGCTGGCGGCTGCAAGCTAACCGACAAGGGCCAGACCTACGCCCGCTCTAT } \\
\text { GACCCTGAACGGCCAGTTCGGCATCATGTACGCCTGGTACTGGCCCAAGGACCAGCCCGCCGACGGCAACCTCGTCAGCGGCCAC } \\
\text { CGCCACGACTGGGAGAATGTCGTCATCTGGTTCAACTCGAACAACGCAAACCAGGCCGGCATCCTGCGCGGTGCGCTTCGGGCC } \\
\text { ACGGCAACTACAAGAAGGTCAACAACCCCCAGCGCAGCGGCAACAACCTCAACGTCGAGTACTTCACCAGCGGCGGCAAGAACC } \\
\text { ACGAGCTGCAGTTCAAGACGTCGCCCGGCCGCACCTACTGGATCTGGGACTGGGACAGGATGGACACCACCGTCCAGGGCGCCCT } \\
\text { CAACCGCGCCGACTTTGGCAGCGCCAACTGCCCCTTCAACAACAACAACTTTGAGAGGAACATGCGCGCCGCGTTTtaa }\end{array}$ \\
\hline $\begin{array}{l}\text { >VL43_VlNLP1_2013 } \\
\text { atgCTTCCCTCCACAATCTTCTCGGTCTTTGCCCTCGTCGGCAGCGCCTTGGCTCAGCATCCCCCCAAGGTTAACCACGACAGTATCA } \\
\text { ACCCCGTCCGCGATACTCTGGGGCCCAACGGCGACATGATCAGGAAGTTCCAGCCTCTGCTTCACATTGCCCACGGTTGCCAGCCT } \\
\text { TACTCCGCTGTCAACACCCGCGGTGAGGTCAAGTAAGACACGCAGCACTCCCTCAGCTTACACAAAGCTCCACGCTAACACATAT } \\
\text { TCTGCAGCGCCGGTCTCCAAGACAGCGGTACCACCGCAGGCGGCTGCAAGGAAACCAGCAAGGGCCAGACCTACGCCCGCTCCAT } \\
\text { GACCCTGAACGGCCAGTTCGGCATCATGTACGCCTGGTACTGGCCCAAGGACCAGCCCGCCGACGGCAACCTCGCCAGCGGCCAC } \\
\text { CGCCACGACTGGGAGAACGTCGTCATCTGGTTCAACTCGAACAACGCAAACCAGGCCGGCATCCTGCGCGGCGCCGCCTCGGGCC } \\
\text { ACGGCGACTACAAGAAGGTCAACAACCCCCAGCGCAACAACAACAACCTCCACGTCGAGTACTTCACCAGCCTCGGCAAGAACCA } \\
\text { CGAGCTGCAGTTCAAGACGTCGCCCGGCCGCACCTACTGGATCTGGGACTGGGACAGGATGGACAGCACCGTCCAGGGCGCCCTC } \\
\text { AACCGCGCCGACTTTGGCAGCGCCAACTGCCCCTTCAACAACAACAACTTTGAGAGGAACATGCGCGCCGCGTTTtaa }\end{array}$ \\
\hline
\end{tabular}

VINLP2 (equal to VdNLP4)

$>$ VL43_VINLP2_2018

atgCAGCACACTCTCCTCTCAACCGCCGCCCTGCTTGGCGCCCTTTCAGCCGTCAATGCTTCTCCTGCGCCCATTCTGAGGCGTGATA TTATCACCGCCCTCCCAGGAAGCGCTGACGAGATCGAGAACAAGTTCCAGCCAATTCTTGACTTCGATACAGACGGCTGTTACAAC ACAGCGGCCATTGACCCTGACGGCAACATAAACCCTGGCAAGGGCGCCACCGGCACTCCTCAGGGCGACTGCCGCGATCCCCCTC AGCTCGAGAACAGCAATGTTTACTCCGGTGGCCGCTGCAACAACGGCGTTTGCGCTATCATGTAAGGCTACACTGATTTGCATGAA GGTACCCCTTTTTGAAACACATCTGACAATAATAGGTACGAGTATTACTTTGAAAAGGACCAGTCGGTCAGCGGCTCGTTTGCGGG CGGCCACCGTCACGACTGGGAAAACGTAGTCGTCTTCGCACGCGGAGACACCATTGTACGTGTTGCGCCATCTTGCCACGGCGGCT ACGGCGGTGCATTGAATGAGTTCCCTGTCGACGGCACGAGCCCGCAGATGGTCTACCACAAGGACAGTGCCGGCACTCACTGCTTT CGCTTCGCCAACGACGCCGACATTGGCGGCGTCGAGAACTTCTCAGGCTCGTTCTACAAGAGCCCGCTCGTCGGATGGCTCAGCTG GCCCAACGAGGGGTTGCGCCAAACCATGTTTGGAGCTTTCAGCGGCGGAGTTGGGCCAAAGTTGGATGATGAGTTTGCGGGCAAG CTTGGTGAGGCAGCTGGTGATGCTGTTCCCGAGTTTGACCCGAATGTCGACGAGtag

$>$ VL32_VINLP2_2018

atgCAGCATACTCTCCTCTCAACCGCCGCCCTGCTTGGCGCCCTGTCAGCCGTCAATGCTTCTCCTGCGCCCATTCTGAGGCGTGATA TTATCACCGCCCTCCCAGGAAACGCCGACGAGATCGAGAACAAGTTCCAGCCAATTCTTGACTTCGATACAGACGGCTGCTACAAC ACAGCTGCCATTGATCCTGACGGCAACACAAACCCTGGCAAGGGCGCCACCGGCACTCCTCAGGGCGACTGCCGCGATCCTCCTC AGCTCGAGAACAGCAATGTTTACTCCCGGCGCCGCTGCAACAACGGCGTCTGCGCTATTATGTAAGGCCACAACATTTTTTCAGGA ACGTGCCCCTTTCTGAAACATAACTGACAATAATAGGTACGAGTATTATTTTGAAAAGGACCAGTCGGTCAGCAGCTCGTTTGCGG GCGGCCACCGTCACGACTGGGAAAACGTAGTCGTCTTCGCACGCGGCGACACTATTGTACGTGTTGCGCCATCTTGCCACGGTGGC TACGGCGGCGCATCGAATGAGTTCCCTGCCGACGGCACGAGCCCGCAGATGGTCTACCACAAGGACAGTGCCGGCACTCACTGCT TCCGCTTCGCCAACGACGCCGACATTGGCGGCGTCGAGAACTTCTCAGGCTCGTTCTACAAGAGCCCGCTCGTCGGATGGCTCAGC TGGCCTAACGAGGGGTTGCGCCAAACCATGCTTGGAGCTTTCAGCGGGGGAGTTGGGCCAAAGTTGGACGACGAGTTTGCAGGCA AGCTTGGTGAGGCAGCTGGTGATGCTGTCCCCGAGTTTGACCCGAATGTCGACGAAtag 
>VL43_VlNLP2_2013

atgCAGCATACTCTCTCTCTCAACCGCCCGCCCTGCTTGGCGCCCTGTCAGCCGTCAATGCTTCTCCTGCGCGCCATTTTGAGGCGTG ATATTATCACCGGCCTTCCCAGAAACGCCGACGAGATTGAGAACCAGTTCCAGCCCATTTTTGACTTTGATACAGACGGGTGGTAC AACACAGCTGCCCATGATCCTGACGGCAACACTAACCCTGGCAAGGGCGCCCCCGGCCCTTCTCAGGGGGGCTGCCCCGATTCTCC TTAACTTGAGAACAACCATGTTTACTTCCGGTGCCGGTGCAACAACGGCGTCTGCGCTATTATGTAAGGCCACCACATTTTTTCAG GAAGGTGCCCCTTTTTGAAACACATCTGACAATAATAGGTACGAGTATTATTTTGAAAAGGACCAGTCGGTCAGCAGCTCGTTTGC GGGCGGCCACCGTCACGACTGGGAAAACGTAGTCGTCTTCGCACGCGGAGACACTATTGTACGTGTTGCGCCATCTTGCCACGGCG GCTACGGCGGTGCATTGAATGAGTTCCCTGTCGACGGCACGAGCCCGCAGATGGTCTACCACAAGGACAGTGCCGGCACTCACTG CTTTCGCTTCGCCAACGACGCCGACATTGGCGGCGTCGAGAACTTCTCAGGCTCGTTCTACAAGAGCCCGCTCGTCGGATGGCTCA GCTGGCCCAACGAGGGGTTGCGCCAAACCATGTTTGGAGCTTTCAGCGGGGGAGTTGGGCCAAAGTTGGACGATGAGTTTGCGGG CAAGCTTGGTGAGGCAGCTGTGATGCTGTCCCGAGTTTGACCCGAATGTTGACGAGtag

\begin{abstract}
$V I N L P 3$ (equal to $V d N L P 5)$
>VL43_VINLP3_2018

atgCTTTTCAGTGTCGGACTTCTCGCCCTCGCGGCCCTGCCTTCGTCTTTCGGAGCTGTCATTCAGGCGCGCCAGGACGACCCCGAGA ACCCTCCCCGTGATCCCCAGCCTCCTCCTCCCGGCCCCATCTTCGGACGGGCACCCGACCTCGACAAGCGCTTCCAGCCTGCTCTTG ACTTTGACACGGACAGCTGCTACAATGTACCAGCAATCGGCCCCAACGGCGACCTCGCCATTGGCATGTATCCCTTCGAGTGGCCG CCTCAAGCGGGCTGCCGCAACGAAGAGATGCTGGACAGAGGCAATGTCTACTCCCGCCAGCGCTGTAACAACGGCTATTGCGTCA TCTTTTACGCCTACTACTTCCAGAAGGATACGGCGACGCCCATTGACGGGCACCGCCACGACTGGGAGCACATTGCCGTGTGGGTG CGCCAGAGCGACAGCTTCGTCACGCACGTTGCCGTCAGCCAGCACAAGGGCTACGACATTCGCGAAAACTCTCAGATCACCTGGA CTGCGGCGGAGAATGGAAAGCCGGCGATTGTCTACCACAAGGACAGCATTCTGACACACTGCTTCCGGTTCGGCAGTGCTGCCGA CGCCGGCGGTCCCGGGCCGGAGAACCACCGGAACCAGTGGATCACGGGCCCGCTTCTGGGGTACTTCGGCTGGGACACTGTCGAG CAGAGGGACAGGATGCTGACGCACAACTGGGAGGCTGGCTCTATCGCCATCAAGAATGAGAACTTTGCTGAGAACATTCGCAAGG CTAGGCCTGCTGGACTGGTCTTTGATGAGAACATTGACGACGAGGGCACCAACAACATCtga
\end{abstract}

$>$ VL32_VINLP3_2018

atgCTTTTCAGTGTCGGACTTCTCGCCCTCGCGGCCCTGCCTTCGTCTTTCGGAGCTGTCATTCAGGCGCGCCAGGACGACCCCGAGA ACCCTCCCCGTGATCCCCAGCCTCCTCCTCCCGGCCCCATCTTCGGACGGGCACCCGACCTCGACAAGCGCTTCCAGCCTGCTCTCG ACTTTGACACGGACAGCTGCTACAATGTACCAGCAATCGGCCCCAACGGCGACCTCGCCATTGGCATGTATCCCTTCGAGTGGCCG CCTCAAGCGGGCTGCCGCAACGAAGAGATGCTGGACAGAGGCAATGTCTACTCCCGCCAGCGCTGTAACAACGGCTATTGCGTCA TCTTTTACGCCTACTACTTCCAGAAGGATACGGCGACGCCCATTGACGGGCACCGCCACGACTGGGAGCACATTGCCGTGTGGGTG CGCCAGAGCGACAGCTTCGTCACGCACGTTGCCGTCAGCCAGCACAAGGGCTACGACATTCGCGAAAACTCCCAGATCACCTGGA CTGCGGCGGAGAATGGCAAGCCGGCGATTGTCTACCACAAGGACAGCATTCTGACACACTGCTTCCGGTTCGGCAGTGGCGCCGA CGCCGGCGGTCCCGGGCCGGAGAACCACAAGAACCAGTGGATCACGGGCCCGCTTCTGGGGTACTTCGGCTGGGACACCGTCGAG CAGAGGGACAGGATGCTGACGCACAACTGGGAGGCTGGCTCTATCGCCATCAAGAACGAGAACTTTGCTGAGAACATTCGCAAGG CTAGGCCTGCTGGGCTGGTCTTTGATGAGAACATTGACGACGAGGGCACCAACAACATCtga

>VL43_VINLP3_2013

atgCTTTTCAGTGTCGGACTCCTCGCCCTTGCGGCTTTGCCTACGTCTTTCGGAGCTGTCATTCAGGCGCGCCAGGACGACCCCGAGA ACCCTCCCCGTGAACCCCAGCCTCCTCCTCCCGGCCCCATCTTCGGACGGGCACCCGACCTCGACAAGCGCTTCCAGCCTGCTCTTG ACTTTGACACGGACAGCTGCTACAATGCACCAGCAATCGGCCCCAACGGCGACCTCGCCATTGGCATGTATCCCTTCGAGTGGCCG CCTCAAGCGGGCTGCCGCAACGAAGAGATGCTGGACAGAGGCAATGTCTACTCCCGCCAGCGCTGTAACAACGGCTATTGCGTCA TCTTTTACGCCTACTACTTCCAGAAGGATACGGCGACGCCCATTGACGGGCACCGCCACGACTGGGAGCACATTGCCGTGTGGGTG CGCCAGAGCGACAGCTTCGTCACGCACGTTGCCGTCAGCCAGCACAAGGGCTACGACATTCGTGAAAACTCCCAGATCACCTGGA CTGCGGCGGAGAATGGAAAGCCGGCGATTGTCTACCACAAGGACAGCATTCTGACACACTGCTTCCGGTTCGGCAGTGGTGCCGA CGCCGGCGGTCCCGGGCCGGAGAACCACAGGAACCAGTGGATCACGGGCCCGCTTCTGGGGTACTTCGGTTGGGACACTGTCGAG CAGAGGGACAGGATGCTGACGCACAACTGGGAGGCTGGCTCTATCGCCATCAAGAATGAGAACTTTGCTGAGAACATTCGCAAGG CTAGGCCTGCTGGGCTGGTCTCTGACGACAACTTTGACGACGAGGGCACGCACAACATCtga

VINLP4 (equal to VINLP9)

>VL43_VINLP4_2018

atgCTTTTCCTGCGAAACATTGCCGTGGTCACCGCCATGGTGCTGTCAGTGCCATCGACGGCCTCCGTCATGCGGCGCCAGAACAAC TCGAGTCGGATCCTCAGCGAGAGTCCAGCCCTCGAACCCATCGTGAACGGCCACGACTTTGCCTACTATTTTGAAGTCAAGTTCCA GCCGCTGGTGGACTTCGACACGGACAGCTGCTACAGCGTGCCCGCCATGACCATGGACGGAACCGCATCCGAGGGCCTTTCGCCC TCCGACGACGTCGGCCCGTGCCGTCCCCGCTCCGCCCTCGACCGGACCAACGTGTACGTCAGGGGCCGCTGCAACCGCGGCTGGTG CGCCTTCGTCTACGCCTACTACTTCCAGATGGACTGGGCCTGGTCGTGGCCCGTCAGCGGCTACAACCACCGCCACGACTGGGAGC ACGTCGTCGTCTGGGCCAAGGAGGGCAAAGTCCGCGGCGTGTCCGTCTCGCACCACGGCGGCTACAAGAGCCGCGTCGCCGAGGA CCAGAGGCTTCGCTTCGACTACACGCCCAAGGAGTTTCCGTATCCTGCGTGGGACCCCATGCCGACGAGCGTCGCCATGCACCCCA AGGTTGTCTTTCACAAGGACGGCGCCCGCACGCACTGCTTCCGCTTCGCCAAGGACAGCGACGACTACGAGGGCCAGGAGAATGA GAGGGGTATTTGGATTCGTGGAGGTCTCGTCAGCATGCTGCTCATGCCGAGCGACTGGCAGGACAAGTTTAGATCTCACGATTGGG AGAGTGCTCACATGGCATGGGCTAATGAGGACGACTTTACCGGCCATCTCGTCAAGTCCATGCCGCAGGAAGCCAGAGACGACGG 
GTTTGACTGTGCCTACGATGAGAATCCTGCTCTCAAAGGCTTTCCCATGGACTGGAAGAAGTGGGACtga

>VL32_VINLP4_2018

atgCTTTTTCTGCGAAACATTGCTGTGGTCACCGCCATGGTGCTGTCAGTGCCATCGACGGCTTCCGTCATGCGGCGCCAGAACAACT CGAGTCGGATCCTCAGCGAGAGTCCAGCCCTCGAACCCATCGTGAACGGCCACGACTTTGCCTACTATTTTGAAGTCAAGTTCCAG CCGCTGGTGGACTTCGACACGGACAGCTGCTACAGCGTGCCCGCCATGACCATGGACGGAACCGCATCCGAGGGCCTTTCGCCCTC CGACGACGTCGGCCCGTGCCGTCCCCGCTCCGCCCTCGACCGGACCAACGTGTACGTCAGGGGCCGCTGCAACCGCGGCTGGTGC GCCTTTGTCTACGCCTACTACTTCCAGATGGACTGGGCCTGGTCGTGGCCCGTCAGCGGCTACAACCACCGCCACGACTGGGAGCA CGTCGTCGTCTGGGCCAAGGAGGGCAAAGTCCGCGGCGTGTCCGTCTCGCAGCACGGCGGCTACGAGAGCCGCGTCGCCGAGGAC CAGAGGCTTCGCTTCGACTACACGCCCAAGGAGTTTCCGTATCCTGCGTGGGACCCCATGCCGACGAGCGTCGCCATGCACCCCAA GGTTGTCTTTCACAAGGACGGCGCCCGCACGCACTGCTTCCGCTTCGCCAAGGACAGCGACGACTACGAGGGCCAGGAGAATGAG AGGGGTATTTGGATTCGTGGCGGTCTCGTCAGCATGCTGCTCATGCCGAGCGACTGGCAGGACAAGTTTAGATCTCACGGTTGGGG GAGTGCTCACATGGCATGGGCTAATGAGGACGACTTTACCGGCCATCTCGTCAAGTCCATGCCGCAGGAAGCCAGAGATGACGGG TTTGACTGTGCCTACGATGAGAATCCAGCTCTCAAAGGCTTTCCCATGGACTGGAAGAAGTGGGACtga

$>$ VL43_VINLP4_2013

atgGTGCTGTCAGTGCCATCGACCGCCTCAGTCATGCGGCGCCAGAGCAACTCGAGTCGGATCCTCAGCGATAGTCAAGCGCTCGAA CCCATCGTGGGCGGCCACGACTTCGCCTACTATTTTGAAGTGAAGTTCCAGCCATTGGTGGACATCGACACCGACAGCTGCTACAG CGTGCCCGCCATGACCATGGACGGAACCGCATCCGAGGGCCTTTCACCCTCCGACGATGTCGGTCCGTGCCGTCCCCGCTCCGCCC TTGACCGGTCCAACGTGTATGTCAGGGGCCGCTGCAACCGCGGATGGTGCGCATTCGTCTACGCCTATTACTTCCAGATGGACTGG GCCTGGTCGTGGCCCGTGAGCAGCTACAACCACCGCCACGACTGGGAGCACGTCGTCGTCTGGGCCAAGGAGGGCAAAGTCCGCG GCGTTTCCGTCTCGCAACACGGCGGCTACGAGAACCGCGTCGCGGAAGACCAGAGGCTTCGCTTCGACTACACACCAAAGGAGTT TCCGTATCCTGCGTGGGATCCCATGCCGACGAGCGTAGCCATGCACCCCAAGGTCGTCTTTCACAAGGACGGTGCCCGCACGCACT GCTTCCGCTTCGCCAAGGACAGCGACGACTATGAGGGCCAGGAGAATGAGAGAGGCGTTTGGATTCGTGGCGGTCTCATCAGTAT GCTGCTCATGCCGAGCGACTGGCAGGAGAAGTTCAGATCTCAGAATTGGGGGAGTGCTCACATGGCATGGGCTAATGAGGAGGAC TTTACCGGGCATCTCGTCAAGTCCATGCCGCAGGAAGCCAGAGACGACGGGTTTGACTGTGCCTACGACGAGAATCCAGCTCTCA AAGGCTTTCCTATGGACTGGAAGAAGTGGGGACtga

VINLP5 (equal to VdNLP10)

$>$ VL43_VINLP5_2018

atgTATCACAAAATCCTTCTCGTCGGCGTGCTAGCCACCCTTACTGGGTTGACTTCCGCCAATGACGCGATCCCAGGCAGTGCTTTCG AAAGTGAGCCAACCCATGAACACAATTACAACACAGATCGCAGTCAACTAACGATCTAAGGACAGCGGACATGAAGCAGCCATC GCCGGTGCTCCCATGTATCACTTTGGTCGCTCGTGGGACCGCAAGCCGTGCTACCCAGAGGCCGGTCAGACTGACGGCGTTAAGAC GGACGGCGTTGACTCGGACCTGTGCTTCAGCAGTCAGAATGGCGGTTGTGCCGATCCCGGACCCTGGAACGGCGTCAACTCTCCCG GAAACCCGTTCCCGGTGTATTACACCGTTCGGCAGTGCAACGACAATGAGTGGCGCGTTGCCTACAGCATCTACTATAAGAAAGAT TCTGGCCATAAGAACGACTGGGAGAACAGCATTGTCATCTGGAACGGAGACGGTGCTGGCGGCTGGAAGCGGAGCGGTACGCTGC TTGGCTGGCACAGCGGCTGGGATTACATTGCTTGGGGCGACATCCAGAACACTGTGAACAAGTAAGTGAAATCCAGGAGATGCAA CTTTTCAAAGCGTCATTGCTGACAAACGCAGCGACGGCGATCTTTTCGACCAGGGAGCCAAGGATCGAAACCATGCCAAGGTTTAT CAGGGATTCTACTATCATGCCACGTTCTCGACTCGCAAGACGTCCCTCAACACCTGCGCCAATACTAGGGACGAGTTCCGATCGAA CGACTGGTACTTCCTTCCTGACGGCACGTGGCTTCACAACGGAGATCTCATCCAAGGTAGGCTGATAACGGAAAGAGTAATACCTT GAGCATCAAAGACTAACCCTGACTCCATCCAGATGGCTGGGATTATGGCTCTGCCGACACGAACCCGTCTTCTCTGCGAAATGAAG CGCGCTGGATCTGCAACCGAGGCtga

$>$ VL32_VlNLP5_2018

atgTATCACAAAATCCTTCTCGTCGGCGTGCTAGCCACCCTTACTGGGTTGACTTCCGCCAATGACGCGATCCCAGGCAGCGCTTTCG AAAGTGAGCCAACCCATGAACACACATACAACACAGATCGCAGTCAACTAACACTCTATAGACAGCGGACATGAAGCAGCCATCG CCGGTGCTCCCATGTATCACTTTGGTCGCTCGTGGGACCGCAAGCCGTGCTACCCAGAGGCCGGTCAGAATGATGGCGTCAAGACG GACGGCGTTGACTCGGACCTCTGCTTCAGCAGTCAGAACGGCGGTTGTGCCGATCCCGGACCCTGGAACGGCGTCAACTCTCCCGG AAACCCGTTTCCGGTGTATTACACCGTTCGGCAGTGCAACGACAATGAGTGGCGCGTTGCGTACAGCATCTACTATAAGAAAGATT CTGGCCATAAGAACGACTGGGAGAACAGCATTGTCATCTGGAACGGAGACGGTGCTGGCGGCTGGAAGCGGAGCGGTACGCTGCT TGGCTGGCACAGCGGCTGGGATTACATTGCTTGGGGCGACATCCAGAACACCGTGAACAAGTAAGCGAAATCCAGGAGATGCAGC TTTTCAAAGCGTTATTGCTGACAAACTCAGCGACGGCGATCTTTTCGACCAGGGAGCCAAGGATCGAAACCATGCCAAGGTTTATC AGGGATTCTACTATCACGCCACGTTCTCGACTCGCAAGACGTCCCTCAACACCTGCGCCAACACTAGGGACGAGTTCCGATCGAAC GACTGGTACTTCCTTCCCGACGGCACGTGGCTTCACAACGGAGATCTCATCCAAGGTAGGCTGATAACGGAAAGAGAAATACCTT GAGCATCAAAGACTAACCCTGACTCCAGATGGCTGGGATTATGGCTCTGCCGACACGAACCCGTCTTCTCTGCGAAATGAAGCAC GCTGGATCTGCAACCGAGGCtga

>VL43_VINLP5_2013

atgTATCACAAAATCCTTCTCGTCGGCGTGCTAGCCACCCTTACTGGGTTGACTTCCGCCAATGACGCGATCCCAGGCAGTGCTTTCG AAAGTGAGCCAACCCAAGAACACAATTACAACACAGATCGCAGTCAACTAACGATCTATAGACAGCGGACAAGAAGCAGCCATC GCCGGTGCTCCCATGTATCACTTTGGTCGCTCGTGGGACCGCAAGCCATGCTACCCAGAGGCCGGTCAGACTGACGGCGTCAAGAC GGACGGCGTTGACTCGGACCTGTGCTTCAGCAGTCAGAATGGCGGTTGTGCCGATCCCGGACCCTGGAACGGCGTCAACTCTCCCG GAAACCCGTTTCCGGTGTATTACACCGTTCGGCAGTGCAACGACAATGAGTGGCGCGTTGCGTACAGCATCTACTATAAGAAGGAT TCTGGCCATAAGAACGACTGGGAGAACAGCATTGTCATCTGGAACGGAGACGGTGCTGGCGGCTGGAAGCGGAGCGGTACGCTGC TTGGCTGGCACAGCGGTTGGGATTACATTGCTTGGGGCGACATCCAGAACACTGTGAACAAGTAAGCAAAATCCAGGAGATGCAG 
CTTTTCAAAGCGTCTTTGCTGACAAACGTAGCGACGGCGATCTTTTCGACCAGGGCGCCAAGGATCGAAACCATGCCAAGGCTTAT CAGGGATTCTACTACCATGCCACGTTCTCGACTCGCAAGACGTCCCTCAACACCTGCGCCAACACTACGGACGAGTTCCGATCGAA CGACTGGTACTTCCTCCCTGACGGCACGTGGCTTCACAACGGAGATCTCATCCAAGGTAGGCTGGTAACGGAAAGAGTAATACGTT GAGCATCAAACACTAACTCTGACTCCATCCAGATGGCTGGGATTAGGGCTCTGCCGACACAAACCCGTCTTCTCTGCCAAATGAGC GCGCTGGATCTGCAACCGAGGCtga

\begin{tabular}{l}
\hline VlNLP6 (equal to VdNLP2) \\
\hline >VL43_VlNLP6_2018 \\
No amplification product \\
>VL32_VlNLP6_2018 \\
No amplification product \\
\hline >VL43_VlNLP6_2013 \\
No information
\end{tabular}

\begin{tabular}{|l}
\hline$V l N L P 7$ (equal to $V d N L P 3$ ) \\
\hline >VL43_VlNLP7_2018 \\
atgGTTTCCAAGATCTTCTCCACCCTCGCTTCCATCGCGCTCGTCGCGGCTGGGCCTGTCAGCCTCCGGGCTGTCGTGCCTCACGACT \\
CGCTGAACCCCGTGAAGCAGGGAGTCCAGACCGGAGCTTTTGGGACGCCATTGCCAAGTCAACCTTTTTCCACATTGCCAAT \\
GGGTGCCAACCCTACACGGCCGTCAAAGAAGCCGGCGATACCCGGTTTGATTTGTCCCAGAAGAATGACCCCGGGAACCCGGAC \\
CGGGTTTTATTTTATTTCGCTCCCCCCCCCCTCGGGCAAACGTGACTGACTTTGCCGGAACCAAACAGCGGCGGTCTCCAGGA \\
CAGTGGCAACATTCTGCGGGTGCAGGGACCAGTCCAAGGGCCAGACCTACGCCCGCGCCAAGGGGTCAACGGGCAGCTGGCC \\
ATCATGTACTCGTTCTACATGCCCAAGGACCAGCCGATCGCGGGCAACGTGGCAGGCGGCCACCGGCACGACTGGGAGAACATTG \\
TCGTCTTCGTCGACGACCCAGCCGCCAACCCGGCACCGGGCATCCTCGGCGGCGCCGCGTCTGGGCACGGCGAGTACAAGAAGAC \\
GGCGACGCCGGACCGCGAGGGCGACAGCGTCAAGGTCGAGTACTTTACGACGTTCCCGACCAACCACGAGCTCCAGTTCACGGCG \\
ACGACGGGCAAGACGTACCCCATCTCGGACTGGGACGCCATGCCCCAGGCGGCGCGCGACGCGCTCGAGACGACCGACTTTGGCA \\
GCGCCAACGTGCCTTTCAAGGACGCCAACTTTGACAGCAACCTCGCCAAGGCGGCGCTCtag \\
>VL32_VINLP7_2018 \\
atgGTTTCCAAGATCTTCTCCACCCTCGCTTCCATCGCGCTCGTCGCGGCTGGGCCTATCAGCCTCCGGGCTGTCGTGCCTCACGACT \\
CGCTGAACCCCGTGACGCAGCGAGTCCAGACCGGAGCTATTGGAGACGCCATTGCCAAGTCAACCCTCTCTCCACATTGCCAAT \\
GGCTGCCAACCCTACACGGCCGTCAACGATGCCGGCGATACCAGGTATGATTGGTCCGCAGATGACTGACCCGGTGAACCCGGAC \\
CGGGTCTTTATCTTTATCTCGCTCCCCCCCCCccTCGGGCAAACGTGACTGACTTTGCCGGAACCAAACAGCGGCGGTCTCCAGG \\
ACAGTGGCAACATTTCTGCGGGGTGCAGGGACCAGTCCAAGGGCCAGACCTACGCCCGCGCCAAGGTGTCAACGGGCAGCTGGC \\
CATCATGTACTCGTTCTACATGCCCAAGGACCAGCCGATCGCGGGCAACGTGGCAGGCGGCCACCGGCACGACTGGGAGAACATT \\
GTCGTCTTCGTCGACGACCCAGCCGCCAACCCGGCACCGGGCATCCTCGGCGGCGCCGCGTCTGGGCACGGCGAGTACAAGAAGA \\
CGGCGACGCCGGACCGCGAGGGCGACAGCGTCAAGGTCGAGTACTTTACGACGTTCCCGACCAACCACGAGCTCCAGTTCACGGC \\
GACGACGGGCAAGACGTACCCCATCTCGGACTGGGACGCCATGCCCCAGGCGGCGCGCGACGCGCTCGAGACGACCGACTTTGGC \\
AGCGCCAACGTGCCTTTCAAGGACGCCAACTTTGACAGCAACCTCGCCAAGGCGGCGCTCtag
\end{tabular}

VINLP8 (equal to VdNLP7)

>VL43_VINLP8_2018

atgACTTCTCTCAGAACGGCCTCCTTCTCCGCTGTGGCGGCTCTGCTCCTGTTGCCGGCAGTCATCGCAACTCCTCTGCCAGACACGC CTCCCACGAAGCTCATCCGCCGCGATCTCCTCCAGCCCCTTGGCGGCTCGGCCTGGTCCGAGCAGGAAAAGTGGTGCCCGGCCCTC GACTACGATACGGACTCGTGCTACAACACGGTCGCCATCTCTCCCTCAGGCCAGCTCAACGCCGGCCAGGACGAGACCAAGTCGG GCGGCGAGATCCTCGGCTGGTGCCGCAAGGAGGTCCGCCTGCAACAGACCAACATTTACGTGCGCTCGCGCTGCAACAACGGCTG GTGCGTGCACATGTACGACTATTACTTCGAGGCCGACTTTGGCTGGGGCGCCCACCGGCACGACTGGGAGCACGTTGCCGTCTGGG TGCAGCACGGCCAGCTCAAGTTCGTCAGCATCTCGCAGCACGGCAAGTGGGACATCCGCATCCTCGACGGCCGCACCGAGGCCCC GCGGTTCGAGCACGGCACCCACCCCAAGGTCGTGTACCACAAGGACGGCGCTCTGACCCACGCCTTCCGCTGGGCCAACGGCGGA GACGAGCCGCCCGAGAACCATTGGAAGAGCTGGCGCTGGGGCGTCGGCGCCGGCCTCATCGAGTGGGAAAGAATGCCGGACAAT CTGCGCAAGACGCTGTCGGCCAAGAATTGGGGCGCGGCCGAGATGGCGGTCCGCGACAAGGACGGAAGCGACTGGAACTTCGCG TGGTACATCAACGAGTCGCAATACTTCTGCTGGGAGACGTATTGCCCCGGCTTCCTCGCTCCCGAGTTCAAGCCGTGGGGTtga

$>$ VL32_VINLP8_2018

atgACTTCTCTCAGAACGGCCTCCTTCTCCGCTGTGGCGGCTCTGCTCCTGTTGCCGGCAGTCATCGCAACTCCTCTGCTCGACACGC CCCACCCGAAGCTCATCCGCCGCGAATCTCCTCCAGCCCCTCGGCGGCTCGGCATGGTCCGAGCAGGAAAAGTGGTGCCCGGCGC 
TCGACTACGATACGGACTCATGCTACAACACGGTCGCCATCTCTCCCTCAGGCCAGCTCAACGCCGGCCAGGACGAGAACAAGCC GGCCGGCGAGATCCTCGGCTGGTGCCGCAAGGAGGTCCACCTGCAACAGACCAATATTTACGTGCGCTCGCGCTGCAACAACGGC TGGTGCGTGCACATGTACGACTACTACTTCGAGGCCGACTTTGGCTGGGGCGCCCACCGGCACGACTGGGAGCACGTTGCCGTCTG GGTGCAGCATGGCCAGCTCAAGTTCGTCAGCATCTCGCAGCACGGCAAGTGGGACATTCGCATCCTCGACGGCCGCACCGACGCC CCGCGGTTCGAGCATGGCACCCACCCCAAGGTCGTGTACCACAAGGACGGCGCTCTGACCCACGCCTTCCGCTGGGCCAACGACG GAGACGAGCCGCCCGAGAACCATTGGAAGAGCTGGCGCTGGGGCGTGGGCGCCGGCCTCATCGAGTGGGAAAGAATGCCGGACA ATCTGCGCAAGACGCTGTCGGCCAAGAATTGGGGCGCCGCCGAGATGGCGGTCCGCGACAAGGACGGAAGCGACTGGAACTTTGC GTGGTACATCAACGAGTCGCAATACTTCTGCTGGGAGACCTATTGCCCCGGCTTCCTCGCTCCCGAGTTCAAGCCGTGGGGTtga

Grey background represents intron

Data Source:

VL43_2018: Sequencing result in this study (Macrogen Europe)

VL32_ 2018: Sequencing result in this study (Macrogen Europe)

VL43_2013: Sequencing result from Malte Beinhoff Ph.D thesis

VL32 V1NLP5 2018

VL43_VINLP5_2018

VL43 VINLP5 2013

VL43 VINLP5 VertiBase

VL_VINLP5_EnsemblFungi_8165

VA VINLP5 EnsemblFungi

VL_VlNLP5_EnsemblFungi_8145

VL $\overline{3} 2$ VlNL $\bar{P} 5$ VertiBase

VD_VINLP5_NCBI

VD_VINLP7_NCBI

VLĀ3_VlNLPי 72018

VL43_VINLP7 VertiBase

VL32 VINLP7 2018

VL32 VINLP7 VertiBase

VL_VİNLP7_EnsemblFungi_8145

VL_VINLP7_EnsemblFungi_8165

VA_VINLP7_EnsemblFungi

VD_VINLP7_NCBI

VL43 VINLP7 2018

VL43_VlNLP7_VertiBase

VL32 VINLP7 2018

VL32 V1NLP7 VertiBase

VL_VINLP7_EnsemblFungi_8145

VL_VINLP7_EnsemblFungi_8165

VA_VINLP7_EnsemblFungi

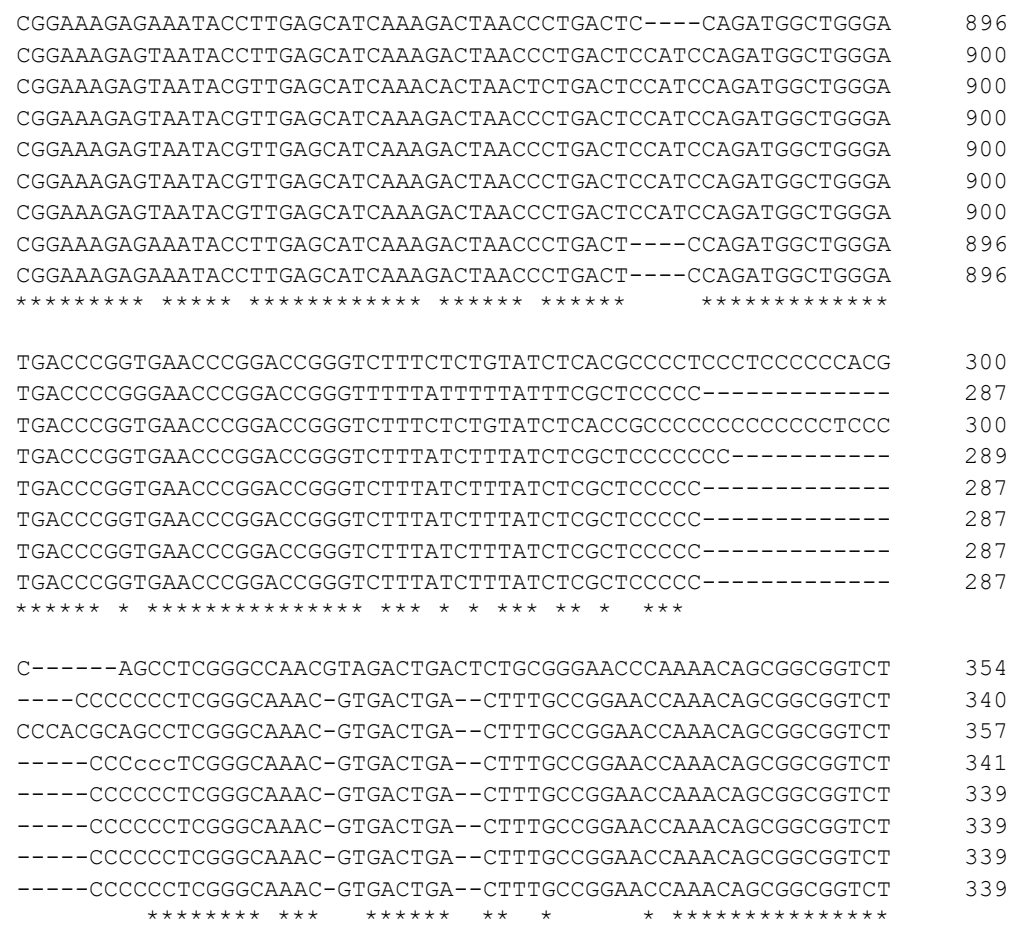

Fig.1 Sequence differences of VINLP5 and VINLP7 among various data sources.VL43_2013 confirmed the NLPs sequences from Malte Beinhoff's thesis. VL43_VertiBase and VL32_VertiBase represent the NLP sequences of Verticillium longisporum (au16) Strain 43 and Verticillium longisporum (au1) Strain 32 from the VertiBase database. VL_EnsemblFungi, VA _EnsemblFungi and VD _NCBI represented the NLP sequences of $V$. longisporum strain VL2, $V$. alfalfae and $V$. dahliae from fungi.ensembl database and NCBI separately.

Data Source:

VL43_ 2018: Sequencing result in this study (Macrogen Europe); VL32_ 2018: Sequencing result in this study (Macrogen Europe); VL43_2013: Sequencing result from Malte Beinhoff Ph.D thesis; VL43_VertiBase: www.vertibase.org Verticillium longisporum (au16), Strain 43, version 1.0; VL32_VertiBase: www.vertibase.org Verticillium longisporum (au1), Strain 32, version 1.0; VL_EnsemblFungi_8145: https://fungi.ensembl.org Verticillium longisporum (GCA_001268145) ASM24013v3; VL_EnsemblFungi_8165: https://fungi.ensembl.org Verticillium longisporum (GCA_001268165) ASM24013v3; VD_NCBI: https://www.ncbi.nlm.nih.gov Verticillium dahliae VdLs.17 GenBank: CP010982.1;VA_ EnsemblFungi : https://fungi.ensembl.org Verticillium alfalfae VaMs.102 (GCA_000150825) ScFostersB_v01 
VL32_2018, VL32_VertiBase and V. dahliae have four nucleotides less in the third intron of VINLP5 compared to others; There are too many successive cytosines in the intron of VlNLP7, so certain region contains variable numbers of cytosines between different sources.

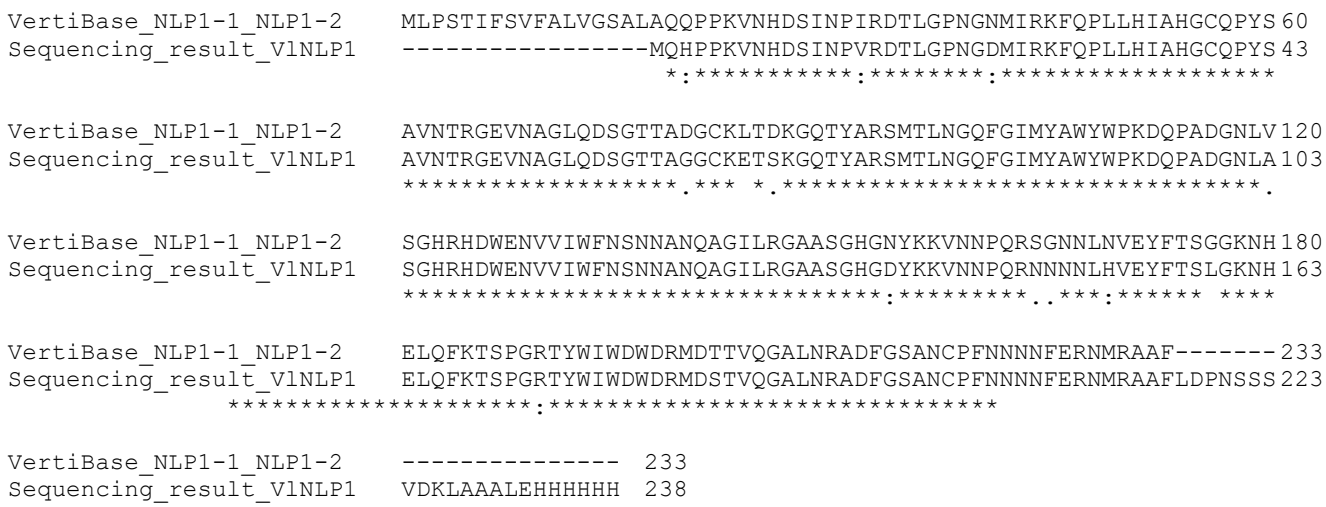

Fig.2 Alignment of VINLP1amino acid sequence between reference sequence from VertiBase and sequencing result in our study. The upper row represents the sequence combined NLP1-1 and NLP1-2 from VertiBase database; the lower row presents the sequence obtained in this study. VINLPI amino acids 1 to 18 predicted as signal peptide was removed from pET21a_VINLP1; in addition, sequencing result amino acids 217 to 238 was the additional sequence from pET21a vector containing His-tag at the end.

As shown in Suppl. Fig.2, the comparison between the amino acid sequence from VertiBase.org and the sequence translated from the sequencing result demonstrated that they represent one protein, although the sequences were not exactly identical.

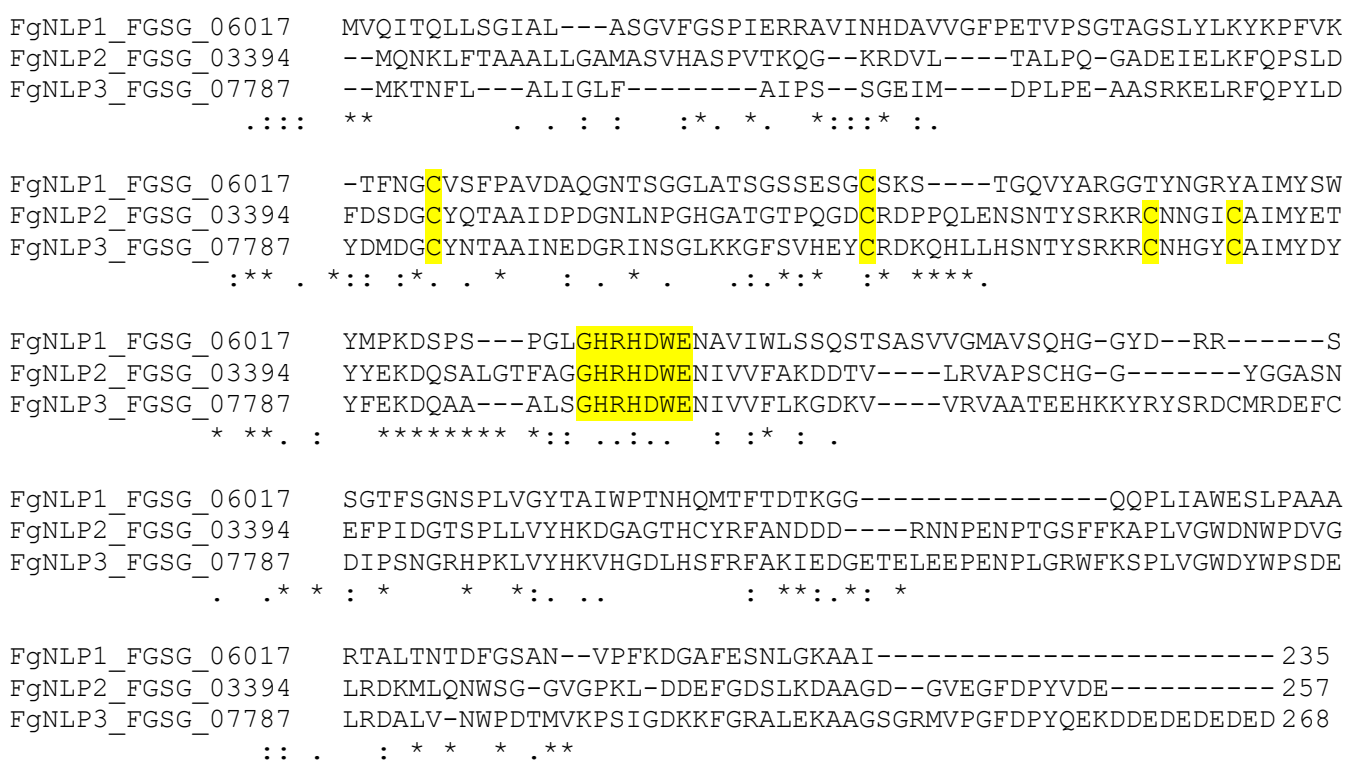

Fig.3 Alignment of three FgNLPs amino acid sequence. Yellow background indicate the conserved features GHRHDWE and cysteine residues. FgNLP1 is type1, FgNLP2 and FgNLP3 are type 2. 
As shown in Suppl. Fig.3, F. graminearum contains three FgNLPs based on the genomic analysis.

FgNLPs contained two NLP types. FgNLP1_FGSG_06017 has one disulfide bridge, whereas FgNLP2_FGSG_03394, FgNLP3_FGSG_07787 have two bridges. Therefore, FgNLP1 is type 1; FgNLP2 and FgNLP3 are type 2.

Table2. Alignment of amino acid sequences of VINLPs and FgNLPs

\begin{tabular}{|c|c|c|c|c|c|c|c|c|c|c|c|c|}
\hline & VINLP5 & VINLP1 & V1NLP7 & NPP1 & FgNLP1 & V1NLP6 & VINLP4 & V1NLP8 & V1NLP3 & FgNLP3 & FgNLP2 & V1NLP2 \\
\hline VL43_2018_V1NLP5_VdNLP10 & 100.00 & 18.27 & 21.29 & 17.82 & 20.00 & 17.50 & 18.50 & 18.34 & 16.52 & 24.07 & 20.66 & 20.66 \\
\hline VL43_2018_VINLP1_VdNLP1 & 18.27 & 100.00 & 52.59 & 34.65 & 34.80 & 35.56 & 24.20 & 22.17 & 24.66 & 25.00 & 25.12 & 24.29 \\
\hline VL43_2018_VINLP7_VdNLP3 & 21.29 & 52.59 & 100.00 & 43.04 & 44.40 & 45.22 & 21.97 & 21.78 & 25.00 & 28.50 & 28.24 & 26.51 \\
\hline NPP1domain & 17.82 & 34.65 & 43.04 & 100.00 & 47.16 & 45.85 & 24.09 & 24.34 & 25.33 & 24.30 & 25.94 & 28.44 \\
\hline FgNLP1_FGSG_06017 & 20.00 & 34.80 & 44.40 & 47.16 & 100.00 & 63.95 & 23.98 & 22.67 & 26.87 & 26.89 & 25.82 & 28.77 \\
\hline VL43_Verti_VINLP6_VdNLP2 & 17.50 & 35.56 & 45.22 & 45.85 & 63.95 & 100.00 & 22.17 & 22.91 & 27.56 & 27.57 & 24.41 & 27.83 \\
\hline VL43_2018_VINLP4_VdNLP9 & 18.50 & 24.20 & 21.97 & 24.09 & 23.98 & 22.17 & 100.00 & 33.73 & 37.16 & 31.75 & 29.67 & 31.84 \\
\hline VL43_2018_VINLP8_VdNLP7 & 18.34 & 22.17 & 21.78 & 24.34 & 22.67 & 22.91 & 33.73 & 100.00 & 37.60 & 34.15 & 34.57 & 39.26 \\
\hline VL43_2018_VINLP3_VdNLP5 & 16.52 & 24.66 & 25.00 & 25.33 & 26.87 & 27.56 & 37.16 & 37.60 & 100.00 & 32.42 & 33.06 & 36.84 \\
\hline FgNLP3_FGSG_07787 & 24.07 & 25.00 & 28.50 & 24.30 & 26.89 & 27.57 & 31.75 & 34.15 & 32.42 & 100.00 & 47.33 & 45.45 \\
\hline FgNLP2_FGSG_03394 & 20.66 & 25.12 & 28.24 & 25.94 & 25.82 & 24.41 & 29.67 & 34.57 & 33.06 & 47.33 & 100.00 & 71.88 \\
\hline VL43_2018_VINLP2_VdNLP4 & 20.66 & 24.29 & 26.51 & 28.44 & 28.77 & 27.83 & 31.84 & 39.26 & 36.84 & 45.45 & 71.88 & 100.00 \\
\hline
\end{tabular}




\section{Article II: Induction of phytoalexins synthesis in oilseed rape by Verticillium longisporum and their detoxification by fungi}

Ling Su, Anna Rathgeb, César Figueroa López, and Petr Karlovsky*

University of Goettingen, Molecular Phytopathology and Mycotoxin Research, 37077 Göttingen, Germany

*Corresponding authors: PK

Emails: pkarlov@gwdg.de

\section{Author contribution:}

Ling $\mathrm{Su}$ designed and carried out all experiments on phytoalexins with pathogens of crucifers. She guided MSc student Cesar Figueroa in experiments with fungi that cannot infect oilseed rape. She processed and integrated the results of Mr. Figueroa in the manuscripts. For HPLC analysis, Dr. Rathgeb helped Ms. Su developing the methods, and operated the machine with Ms. Su together. Ms. Su processed the data from in vitro experiments. The samples from $\mathrm{Cu}$-treated plants were analyzed by Dr. Rathgeb; Ms. Su combined these results with her and Mr. Figueroa's results and wrote the entire manuscript. 


\section{Abstract}

Verticillium longisporum is a soilborne vascular pathogen infecting oilseed rape and other crucifers. Phytoalexins are plant defense metabolites induced by pathogen infection that are toxic to microorganisms. This study describes the induction of phytoalexins in oilseed rape plants by $V$. longisporum infection, the toxicity of brassinin, brassilexin and cyclobrassinin to V. longisporum, and the ability of Verticillium species to detoxify these phytoalexins.

We found that infection of $B$. napus with $V$. longisporum induced the synthesis of phytoalexins brassilexin, spirobrassinin, rutalexin, and cyclobrassinin, which were found in extracts of whole shoots as well as in xylem sap. All four phytoalexins also accumulated in plants treated with copper sulfate. V. longisporum but also V. dahlie and V. albo-atrum, which do not infect crucifers, degraded brassilexin more efficiently than S. sclerotiorum, R. solani and L. maculans, which are pathogens of crucifers. Verticillium species pathogenic to crucifers did not possess higher degradation activities than species that do not infect crucifers. Brassilexin was degraded by Verticillium spp. to 3-(amino)methylenindoline-2-thione, which was further degraded to unknown products. Alternaria alternata, Aspergillus nidulans and Fusarium graminearum degraded brassilexin to the same product at a lower rate. S. sclerotiorum but no other fungi produced a glucose conjugate of brassilexin. Verticillium spp. degraded brassinin at a lower rate than other pathogens of crucifers. Brassilexin at a concentration of $20 \mu \mathrm{g} / \mathrm{ml}$ completely inhibited the germination of $V$. longisporum spores; brassinin and cyclobrassinin have not inhibited spore germination. None of the three phytoalexins at a concentration of $40 \mu \mathrm{g} / \mathrm{ml}$ inhibited the growth of $V$. longisporum. 


\subsection{Introduction}

\subsubsection{Oilseed rape (Brassica napus L.)}

The family Brassicaceae, commonly called crucifers, consists of about 340 genera and 3,709 species worldwide. This family includes many plants with importance in human nutrient, animal feeding and industrial utilities. Among the cash crops belonging to the genus Brassica, Brassica napus (oilseed rape) occupies a global important position. Oilseed rape is the second most important oilseed plant after soybean (USDA 2019).

Winter type and spring type of oilseed rape are cultivated in Europe, North America and Asia (Janick 2002). Winter type B. napus requires vernalization in order to induce stem elongation and flowering, but spring type does not. Brassica napus (AACC genome, $2 \mathrm{n}=38$ ) is derived from a hybridization between Brassica rapa (AA genome, 2n=20) and Brassica oleracea (CC genome, 2n=18) (Koh et al. 2017).

Due to a long-term extensive cultivation of oilseed rape on large areas, the crop is threatened by various disease, most important among which are caused by fungal infection. The fungal diseases with the largest economic impact include Sclerotinia stem rot (Sclerotinia sclerotiorum), blackleg (Leptosphaeria maculans and Leptosphaeria biglobosa), clubroot (Plasmodiophora brassicae), Alternaria blight (Alternaria brassicae), light leaf spot (Pyrenopeziza brassicae), grey mold (Botrytis cinerea) and Verticillium stem striping (Verticillium longisporum) (Dunker et al. 2008; Clarkson et al. 2014; Strehlow et al. 2015; Cai et al. 2018).

\subsubsection{Phytoalexins of crucifers}

Phytoalexins are plant secondary metabolites that are synthesized de novo by host plants infected with a pathogen and display antimicrobial activity (Kuć and Rush 1985). Abiotic stress, such as exposure to heavy metal salts or UV radiation, may also elicit phytoalexin synthesis in plants. Phytoalexins are usually not produced by healthy plants, which differentiates them from phytoanticipins (VanEtten et al. 1994). Because phytoalexins might harm plant cells as well, their biosynthesis is limited to the tissues experiencing stress. 
Over 30 Brassica species are capable of producing cruciferous phytoalexins with indole as a common precursor. Takasugi et al. (1986) reported the first three phytoalexins in crucifer plants, brassinin, 1-methoxybrassinin and cyclobrassinin. After three decades, fifty-four kinds of cruciferous phytoalexins have been characterized as listed in figure 2. The spectrum of phytoalexins varies with plant species, and also depends on the type of stress, to which the plant is exposed, for example on the pathogen species.

Brassinin is the first naturally occurring phytoalexin reported to be produced from mainly Brassica spp.; a common biosynthetic precursor of several other phytoalexins known to contains a dithiocarbamate group attached to a 3-methylindol moiety, which produces an insecticide/herbicide effects (Pedras and Abdoli 2017; Pedras et al. 2011b). Brassilexin is among the most potent antifungal sulphur-containing phytoalexin from Brassica spp.. Cyclobrassinin has been found to accumulate in several Brassica spp. tissue when elicited with Pseudonomas cichorri and Alternaria brassicae.

A review reported five phytoalexins produced by oilseed rape, brassilexin, cyclobrassinin, cyclobrassinin sulfoxide, 1-methoxybrassinin and spirobrassinin (Pedras et al. 2011).

\subsubsection{Detoxification of phytoalexins by pathogens}

Crucifer plants and their pathogens coexisted over a long period of time. During this coevolution, some of the pathogens developed a capability to metabolize cruciferous phytoalexins into nontoxic compounds. Although the benefit of the pathogens from phytoalexin detoxification has not been rigorously proven in most cases (Soledade et al. 1993), it is assumed that detoxifiation abilities developed during the adaptation of pathogens to their hosts and that these activities improve fitness of the pathogen in host plants. For instance, the best-studied phytoalexin brassinin can be metabolized by $L$. maculans isolates virulent on oilseed rape, L. biglobosa, A. brassicicola and $S$ sclerotiorum.

Pathogens of crucifers produce a variety of enzymes involved in many types of detoxification, such as oxidation, hydrolysis, reduction or even conjugation with glucose (Pedras et al. 2011b). They detoxify phytoalexin via enzymatic transformation into non-toxic metabolites to some intermediate products which were shown to be very similar to phytoalexins (i.e. indole-3carboxaldehyde), then further metabolized to nontoxic products (Pedras and Abdoli 2017; Pedras 
et al. 2011b; Pedras et al. 2009a; Soledade et al. 1993; Rouxel et al. 1991). Pedras and Abdoli reported that Leptosphaeria maculans (oilseed rape virulent isolate) and L. biglobosa isolates detoxified brassilexin via 3-aminomethyleneindole-2-thione, to the polar metabolite 3formylindolyl-2-sulfonic acid and Sclerotinia sclerotiorum to a glucosyl derivative (Pedras and Abdoli 2017; Pedras and Suchy 2005; Rouxel et al. 1991). It was reported also that Leptosphaeria maculans, Rhizoctonia solani and both Alternaria brassicola and A. brassicae were able to transform and detoxify several cruciferous phytoalexins including cyclobrassinin to non-toxic glucosyl and hydroxyl derivatives (Pedras and Abdoli, 2016; Pedras et al., 2004, 2011c, 1998; Dahiya and Rimmer, 1988). Main detoxification products of brassilexin, brassinin and cyclobrassinin were summarized in Figure 12, 15 and 18.

\subsubsection{Degradation of phytoalexins and fungal virulence}

Soledade et al. (1993) reported that the virulent and avirulent strains of L. maculans utilize different brassinin detoxification reactions that fundamentally affect brassinin degradation efficiency. It indicates that fungal capability of degrading phytoalexins is related to virulence. The virulent isolates requires only $24 \mathrm{~h}$ to degrade brassinin whereas avirulent isolate required more than 5 days (Fig. 1). Virulent L. maculans isolates transform brassinin rapidly via intermediate (compound 6 in Fig. 1), while avirulent isolates convert brassinin much slower via different intermediates (compound 2 and 3 in Fig. 1). However, the finally less toxic degradation products are the same in virulent and avirulent isolates, which include an aldehyde (compound 4 in Fig. 1) and an acid (compound 5 in Fig. 1).

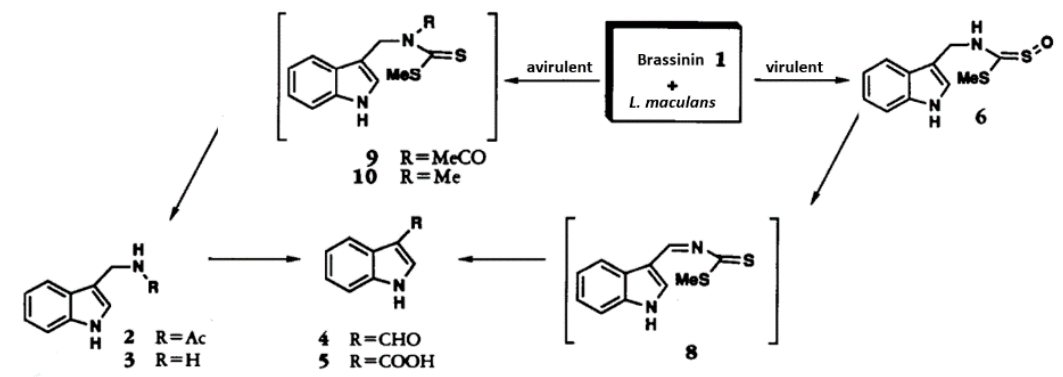

Fig. 1 Catabolism of brassinin by virulent and avirulent isolate of $\boldsymbol{L}$. maculans. Virulent $L$. maculans isolates transform brassinin rapidly via intermediate 6 , while avirulent isolates convert brassinin much slower via different intermediates 2 and 3. But the final less toxic degradation products are identical in virulent and avirulent isolates, which include the aldehyde 4 and acid 5. Figure is modified based on Soledade et al. (1993). 


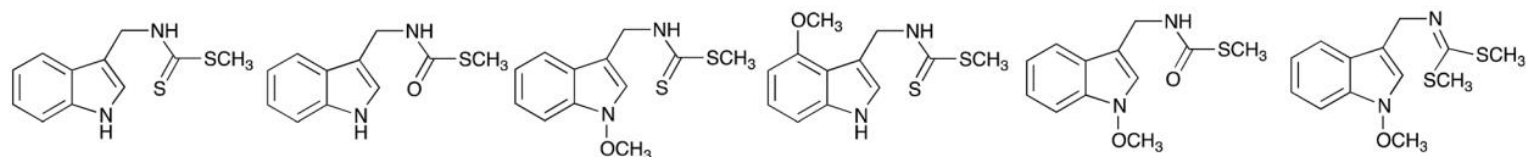

brassinin (1) $\quad$ brassitin (2) 1-methoxybrassinin (3) 4-methoxybrassinin (4) 1-methoxybrassitin (5) 1-methoxybrassenin A (6)<smiles>COn1cc(C(=O)/N=C(\C)SC)c2ccccc21</smiles>

1-methoxybrassenin B (7)<smiles>CON1C(=O)/C(=C/N=C(S)SC)c2ccccc21</smiles>

wasalexin $\mathrm{A}(\mathbf{8})$

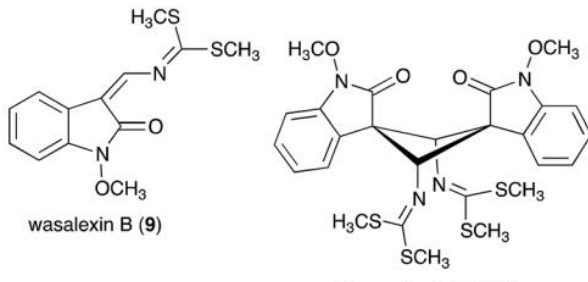

biswasalexin $\mathrm{A} 1$ (10)

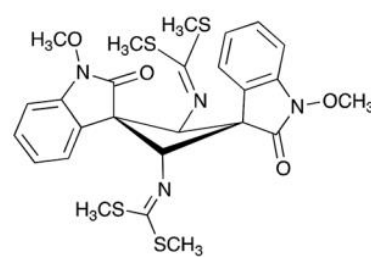

biswasalexin A2 (11)<smiles>CSC1=NCc2c([nH]c3ccccc23)S1</smiles><smiles>CS(=O)C1=NCc2c([nH]c3ccccc23)S1</smiles>

cyclobrassinin (12) cyclobrassinin sulfoxide (13)<smiles>COn1c2c(c3ccccc31)CN=C(S(C)=O)S2</smiles>
sinalbin A (14)<smiles>COn1c2c(c3ccccc31)CN=C(SC)S2</smiles>
sinalbin $\mathrm{B}(\mathbf{1 5})$<smiles>COc1cccc2[nH]c3c(c12)CN=C([SeH])S3</smiles><smiles>COc1cccc2nc3sc(SC)ncc-3c12</smiles><smiles>Cn1c(=O)sc2[nH]c3ccccc3c2c1=O</smiles>

4-methoxy cyclobrassinin (16) dehydro cyclobrassinin (17)

\section{4-methoxy} dehydrocyclobrassinin (18) rutalexin (19)<smiles>c1ccc2c(c1)[nH]c1sncc12</smiles>

brassilexin (20)<smiles>COn1c2ccccc2c2cnsc21</smiles>

sinalexin (21)<smiles>CON1c2ccccc2C2(CN=C(SC)S2)N(OC)c2ccccc2C12CN=C(SC)S2</smiles>
dioxibrassinin (22) (S)-spirobrassinin (23)

\section{$(R)$-1-methoxy
spirobrassinin (24)} $\begin{array}{ll}\text { 1-methoxy } & (2 R, 3 R)-1-\text { methoxyspiro } \\ \text { spirobrassinol (25) } & \text { brassinol methyl ether (26) }\end{array}$

erucalexin (27)<smiles>Cc1[nH]c2ccccc2c1C=O</smiles>

brassicanal A (28)<smiles>COC(=O)c1cn(OC)c2ccccc12</smiles>

methyl 1-methoxyindole3-carboxylate (34)<smiles>C[C@@]1(O)CSc2c(C=O)c3ccccc3n21</smiles>
brassicanal B (29)<smiles>c1ccc2c(-c3nccs3)c[nH]c2c1</smiles>

camalexin (35)

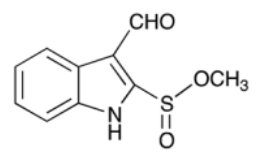

brassicanal C (30)<smiles>COc1ccc2c(-c3nccs3)c[nH]c2c1</smiles>

6-methoxycamalexin (36)

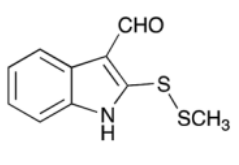

caulilexin A (31)<smiles>Cn1cc(-c2nccs2)c2ccccc21</smiles>

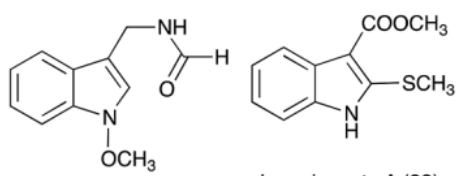

caulilexin B (32)

brassicanate $\mathrm{A}$ (33)<smiles>N#CCc1c[nH]c2ccccc12</smiles>

indolyl-3acetonitrile (38)<smiles>COn1cc(CC#N)c2ccccc21</smiles>

caulilexin C (39)<smiles>COc1cccc2[nH]cc(CC#N)c12</smiles>

arvelexin (40)

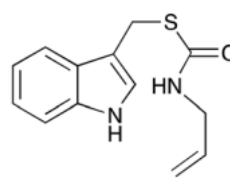

brussalexin $\mathrm{A}$ (41)<smiles>COc1cccc2[nH]cc([N+](=O)[O-])c12</smiles>

rapalexin $\mathrm{A}(\mathbf{4 2})$<smiles>COc1c(O)ccc2[nH]cc([N+](=O)[O-])c12</smiles><smiles>COc1cccc2c1C(=O)C(=O)N2</smiles>

rapalexin $B(43) \quad$ isalexin (44) 


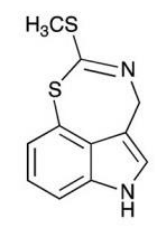

cyclonasturlexin (45)

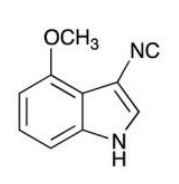

isocyalexin $\mathrm{A}(46)$

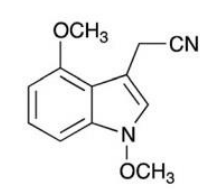

tenualexin (47)

$\mathrm{H}_{3} \mathrm{C}$
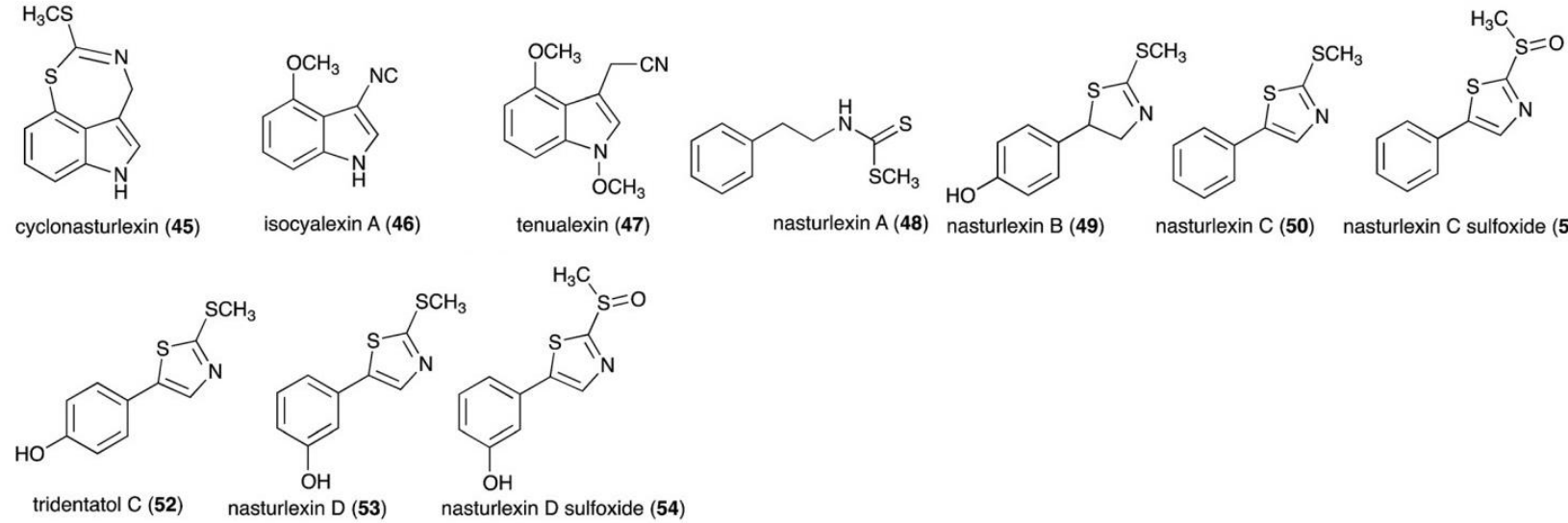

nasturlexin A (48) nasturlexin B (49)

nasturlexin $\mathrm{C}(\mathbf{5 0})$

nasturlexin $\mathrm{C}$ sulfoxide $(\mathbf{5 1})$

Fig. 2 Chemical structures of 54 known cruciferous phytoalexins. Source of chemical structures: Pedras and Abdoli (2017).

\subsubsection{Metabolic profiling of xylem sap from oilseed rape infected by $V$. longisporum}

Dr. Astrid Ratzinger's study revealed that Verticillium longisporum infection affects 19 metabolites in the xylem sap of Brassica napus via nontargeted metabolic profiling. Among them, certain metabolites were only detected in rapeseed plants infected with $V$. longisporum, but not in the mock plants. Considering this property, it was suggested that these metabolites might be phytoalexins (Ratzinger 2008). Dr. Mareike Possienke's metabolic fingerprinting research indicated that after infection with $V$. longisporum, some phytoalexins were induced in B. napus, such as cyclobrassinin (Possienke 2012). Dr. Husam Ibrahem Aroud further suggested that one of the purified compounds from B. napus elicited by $V$. longisporum can be a phytoalexin detoxification product of a cruciferous plant (Aroud 2013).

However, none of these studies continued to conduct further and more specialized research on phytoalexins. In this study, we clarify the phytoalexins produced by B. napus after $V$. longisporum infection and whether the fungal aggressiveness to cruciferous plants affects their phytoalexin degradation efficiencies. 


\subsection{Methods and materials}

\subsubsection{Chemicals, plant material and fungal strains}

Brassinin standard was purchased from Axon Medchem BV (Hanzeplein, the Netherlands). Brassilexin standard was purchased from Toronto Research Chemicals (Toronto, Canada). Cyclobrassinin was synthetized according to Csomos et al. (2005) and purified by preparative HPLC (purity 98\%). Rutalexin standard was synthesized according to Budovska et al. (2015) and purified by preparative TLC (purity $>85 \%$ ). An attempt to synthesize spirobrassinin according to Budovska (2014) lead to a mixture of three isomers. Because the amounts of rutalexin and spirobrassinin were not sufficient for degradation experiments, the compounds were only used for the identification of these metabolites in plant samples by HPLC-MS. Working solutions of phytoalexins were prepared in methanol. Acetonitrile, methanol and other solvents were HPLC grade.

Rapid-cycling Brassica napus (oilseed rape or canola) (SKU: 5-001, Wisconsin-fast plants, RAPID-CYCLING BRASSICA COLLECTION, University of Wisconsin-Madison, Department of Plant Pathology, Wisconsin, USA) and a Verticillium longisporum susceptible cultivar Falcon (Norddeutsche Pflanzenzucht, Hans-Georg Lembke KG, Hohenlieth, Germany) were used in this study. Seeds were surface sterilized in $70 \%$ of ethanol for $5 \mathrm{~s}$ and rinsed in sterile tap water for 30s twice.

After surface sterilization, seeds were pre-germinated in sterile silica sands, which were kept in climate chamber with $16 \mathrm{~h}$ light and $8 \mathrm{~h}$ darkness at a temperature of $25^{\circ} \mathrm{C}$ (day) $/ 22^{\circ} \mathrm{C}$ (night).

Nine isolates of Verticillium species and four other cruciferous fungal pathogens were used (Table 1), which were kindly provided by Prof. A. von Tiedemann (Plant Pathology and Crop Protection, Department of Crop Sciences, Georg-August-University Göttingen).

For long-term storage of Verticillium species, pure cultures were grown in potato extract glucose broth (PDB) medium (Carl Roth $\mathrm{GmbH}$, Karlsruhe, Germany) and incubated at $23^{\circ} \mathrm{C}$ under constant shaking $(130 \mathrm{rpm})$ at $23^{\circ} \mathrm{C}$ in the dark for 14 days. Conidia suspensions were prepared by filtering the fungal culture through double layers of sterile cotton gauze (Verbandmull $\mathrm{ZZ}$, Hartmann, Northeim, Germany) and the concentration of spores suspension were determined 
under a microscope by using a haemocytometer. Medium of suspension was removed after centrifugation at 5,000 rpm for $10 \mathrm{~min}$, and conidia stock concentrations were adjusted to $1 \times 10^{7}$ conidia/ml by re-suspending the pellet in sterile demineralized water containing $20 \%$ of glycerin. The aliquots were stored at $-80^{\circ} \mathrm{C}$.

Table 1 Fungal strains used in this study

\begin{tabular}{|c|c|c|c|c|c|c|c|}
\hline $\begin{array}{l}\text { Name used } \\
\text { in this study }\end{array}$ & Name & $\begin{array}{l}\text { Gö } \\
\text { Name }\end{array}$ & Species & Lineage & $\begin{array}{l}\text { Virulence } \\
\text { on } \\
\text { crucifers }\end{array}$ & $\begin{array}{l}\text { Original } \\
\text { plant host }\end{array}$ & Geographical origin \\
\hline \multicolumn{8}{|c|}{ Verticillium species } \\
\hline VL43 & VL43 & IPP121 & V. longisporum & $\mathrm{A} 1 \mathrm{xD} 1$ & + & $\begin{array}{l}\text { Brassica } \\
\text { napus }\end{array}$ & Mecklenburg/Germany \\
\hline VL1191 & PD402 & IPP1191 & V. longisporum & $\mathrm{A} 1 \mathrm{xD} 2$ & - & $\begin{array}{l}\text { Armoracia } \\
\text { rusticana }\end{array}$ & Illinois/USA \\
\hline VL1199 & PD356 & IPP1199 & V. longisporum & $\mathrm{A} 1 \mathrm{xD} 2$ & - & $\begin{array}{l}\text { Armoracia } \\
\text { rusticana }\end{array}$ & Illinois/USA \\
\hline VL1194 & PD589 & IPP1194 & V. longisporum & A1xD3 & + & $\begin{array}{l}\text { Brassica } \\
\text { oleracea }\end{array}$ & Gunma/ Japan \\
\hline VL1197 & PD687 & IPP1197 & V. longisporum & A1xD3 & - & $\begin{array}{l}\text { Armoracia } \\
\text { rusticana }\end{array}$ & Niedersachsen/Germany \\
\hline VL32 & VL32 & IPP0119 & V. longisporum & $\mathrm{A} 1 \mathrm{xD} 3$ & - & $\begin{array}{l}\text { Brassica } \\
\text { napus }\end{array}$ & Mecklenburg/Germany \\
\hline VL1190 & $\begin{array}{l}\text { PD322 } \\
\text { (Ls.17) }\end{array}$ & IPP1190 & V. dahliae & $\begin{array}{l}\text { Main } \\
\text { Group }\end{array}$ & - & $\begin{array}{l}\text { Lactuca } \\
\text { sativa } L \text {. }\end{array}$ & Watsonville/USA \\
\hline VL1192 & PD502 & IPP1192 & V. dahliae & D2 & - & Acer sp. & Greenfield/USA \\
\hline Va1584 & PD693 & IPP1584 & V. albo-atrum & & - & Potato & UK \\
\hline \multicolumn{8}{|c|}{ Other cruciferous pathogens } \\
\hline Ss1328 & Ss 1328 & IPP1328 & $\begin{array}{l}\text { Sclerotinia } \\
\text { sclerotiorum }\end{array}$ & & + & $\begin{array}{l}\text { Brassica } \\
\text { napus }\end{array}$ & Saskatoon/Canada \\
\hline Ss62 & Ss62 & & $\begin{array}{l}\text { Sclerotinia } \\
\text { sclerotiorum }\end{array}$ & & & & \\
\hline Rs1081 & $\begin{array}{l}\text { Rs } 108 \\
1\end{array}$ & IPP1081 & $\begin{array}{l}\text { Rhizoctonia } \\
\text { solani }\end{array}$ & $\begin{array}{lcc}\text { AG } & 2-1 \quad & / \\
\text { IfZ } & 0235 \\
\text { r5 } & \end{array}$ & + & & \\
\hline Lm0749 & $\begin{array}{l}\text { Lm074 } \\
9\end{array}$ & IPP0749 & $\begin{array}{l}\text { Leptosphaeria } \\
\text { maculans }\end{array}$ & & + & $\begin{array}{l}\text { Brassica } \\
\text { napus }\end{array}$ & Canada \\
\hline
\end{tabular}

Gö Name represents the names used for fungal strains collections in Prof. A. von Tiedemann group. + represents virulent, - represents avirulent Data source: Novakazi et al. (2015)

Fungal strains were provided by Prof. A. von Tiedemann (Plant Pathology and Crop Protection, Department of Crop Sciences, Georg-August-University Göttingen).

\subsubsection{Plants infection assay}

$150 \mu \mathrm{l}$ of conidia stock suspension of $V$. longisporum (VL43) $\left(10^{5}\right.$ to $10^{7}$ conidia/ml) were pipetted into $50 \mathrm{ml}$ sterile PDB medium and under constant shaking condition at $23^{\circ} \mathrm{C}, 130 \mathrm{rpm}$ in the dark for 14 days. Conidia suspension were filtered through sterile cotton gauze and concentrated by centrifugation at 4,500 rpm for $3 \mathrm{~min}$. The final concentration for inoculation was adjusted to $1 \times 10^{7}$ conidia/ml with sterile tap water. 
The roots of approximate two-week-old seedlings of oilseed rape were dipped into the conidia suspension for $45 \mathrm{~min}$ (ZEISE and Tiedemann 2002b, 2002a). Instead of conidia suspension, roots of plants used for control were immersed in sterile tap water. Afterwards, the seedlings were transplanted into $11 \times 11 \times 12 \mathrm{~cm}$ pots containing sterile compost soil: sand : turf mixture (2:1:1). Inoculated plants were kept in climate chamber with $16 \mathrm{~h}$ light and $8 \mathrm{~h}$ darkness at a temperature of $25^{\circ} \mathrm{C}$ (day) $/ 22^{\circ} \mathrm{C}$ (night).

\subsubsection{Collection of xylem sap from B. napus plants}

Scholander pressure chamber (Scholander et al. 1965) was used for collecting xylem sap from rapid cycling oilseed rape plants infected by VL43 at 42 days post inoculation (dpi) (Scholander et al. 1965; Ratzinger et al. 2009). Xylem sap of control samples were collected together. Before extraction, soil on the roots was washed off under running tap water. Stem of oilseed rape with its attached roots was inserted into the Scholander pressure chamber. The xylem sap was taken by collecting the gushed out liquid from the top of the stem. The used pressure during the process has not exceed 1.5 MPa. Collected xylem sap was stored at $-20^{\circ} \mathrm{C}$.

\subsubsection{Abiotic and biotic elicitation of phytoalexins in B. napus}

Eighteen 42-day-old seedlings of $B$. napus cultivar Falcon were sprayed with $10 \mathrm{mM} \mathrm{CuSO}_{4}$ solution (abiotic elicitor) containing 0.5\% TWEEN80 (HARPER and BERKENKAMP 1975). After 7 days, the leaves were harvest and rinsed up under running water for removing the rest of $\mathrm{CuSO}_{4}$. After lyophilization, the leaves were stored at $-20^{\circ} \mathrm{C}$.

Nineteen-day-old oilseed rape seedlings were root-dipping inoculated with $V$. longisporum (VL43) or sterile tap water. The leave samples were collect at $30 \mathrm{dpi}$, lyophilized and stored at $20^{\circ} \mathrm{C}$.

\subsubsection{Extraction of secondary metabolites}

Lyophilized leaf-samples were grinded and homogenized at room temperature. $200 \mathrm{mg}$ of leafpowder were suspended in $3 \mathrm{ml}$ acetone/water $(9: 1 \mathrm{vol} / \mathrm{vol})$ and incubated under shaking condition for $45 \mathrm{~min}$. After centrifugation at full speed for $10 \mathrm{~min}$, the supernatant was 
transferred into a new tube. The solvent was removed using a speed vacuum concentrator (RVC 2-25 CD plus, Christ, Osterode am Harz, Germany) at $30^{\circ} \mathrm{C}$. The samples were stored at $-20^{\circ} \mathrm{C}$.

Just prior to chemical analysis by HPLC-MS-QTOF, the samples were dissolved in methanol/water (1:1, vol./vol.) and incubated at room temperature for $30 \mathrm{~min}$. Subsequently, the samples were centrifuged at $8.000 \mathrm{x}$ g for $10 \mathrm{~min}$ to remove granular impurities.

\subsubsection{Degradation of phytoalexins by fungi}

For degradation analysis, pure cultures of Verticillium species were grown in $500 \mu 1$ of minimal medium (J.W. Bennett, L.L. Lasure (Eds.) 1991). A final conidia concentration of $2 \times 10^{5}$ conidia/ml was adjusted using water. An agar plug of Rhizoctonia solani or Leptosphaeria maculans containing fungal mycelia was placed into $500 \mu \mathrm{l}$ minimal media. Sclerotinia sclerotiorum was grown on PDA; half of a sclerotium was used for degradation assay.

To investigate the degradation ability of the tested fungi (Table 1), $5 \mu$ l of stock solutions of brassinin, brassilexin or cyclobrassinin were added to $500 \mu \mathrm{l}$ fungal cultures to a final concentration of $20 \mu \mathrm{g} / \mathrm{ml}$. To exclude fungal metabolites, $5 \mu \mathrm{l}$ of methanol were used as control. Five hundred $\mu \mathrm{l}$ of minimum medium without fungi were used to testing spontaneous degradation of phytoalexins. Three or six replicates were performed.

Samples for analysis were taken at $30 \mathrm{~min}, 6 \mathrm{~h}, 20 \mathrm{~h}$ and $72 \mathrm{~h}$ after adding phytoalexin into cultures. For brassinin degradation analysis, additional samples were collect at $144 \mathrm{~h}$. Forty-five microliter of culture supernatant was transferred into a new $1.5 \mathrm{ml}$ Eppendorf tube containing $45 \mu \mathrm{l}$ of acetonitrile. After centrifugation at full $8,000 \mathrm{x} \mathrm{g}$ for $10 \mathrm{~min}$, the samples were transferred into HPLC glass vials and stored at $-20^{\circ} \mathrm{C}$ until analysis.

\subsubsection{Analysis of phytoalexins and their degradation products by HPLC}

Samples were analysed via HPLC-QTOF-MS using models 6545 for QTOF and 1290 Infinity II for HPLC from Agilent (Darmstadt, Germany). These instruments were equipped with a quaternary pump, an injector, a diode array detector (wave length range 190-400 nm), a quadruple time of flight detector (spectra range 100-1700 $\mathrm{m} / \mathrm{z}$ ), and a degasser. 
HPLC-DAD analysis was carried out on Agilent Zorbax Eclipse Plus C18 column (1.8 $\mu \mathrm{m}$ particle size, $2.1 \mathrm{~mm}$ diameter and 50mm length), eluted with a gradient of distilled $\mathrm{H}_{2} \mathrm{O}(\mathrm{A})$ and $\mathrm{MeOH}$ (B) both acidified with $0.1 \%$ LC-MS formic acid: from 95\% to 5\% A in 4 min, hold for $0.5 \mathrm{~min}$ and then re-equilibrate to $95 \%$ A for $3.5 \mathrm{~min}$. The flow rate was $0.4 \mathrm{~mL} / \mathrm{min}$. HPLCQTOF method: Varian Polaris $3 \mathrm{C} 18$ Ether column $(3 \mu \mathrm{m}$ particle size, $2 \mathrm{~mm}$ diameter and 50 mm length) was eluted with a gradient of distilled $\mathrm{H}_{2} \mathrm{O}(\mathrm{A})$ and $\mathrm{MeOH}(\mathrm{B})$, both acidified with $0.1 \%$ formic acid: From $95 \%$ to $5 \% \mathrm{~A}$ in $20 \mathrm{~min}$, hold for $2.5 \mathrm{~min}$ and then re-equilibrated to $95 \%$ A for $7.5 \mathrm{~min}$. The flow rate was $0.2 \mathrm{~mL} / \mathrm{min}$. Temperature was set to $35^{\circ} \mathrm{C}$ and sample injection contained $10 \mu \mathrm{L}$. A solvent blank was measured after every fifth sample to keep the system clean and the mixture of the authentic purified phytoalexins was measured after every blank to be use as a reference point. Eluted compounds were monitored via the Agilent working station through an electrospray ionization source from the same instrument, which operated in positive ionization mode. The sheath gas temperature was set to $350{ }^{\circ} \mathrm{C}$ and the flow to $11 \mathrm{~L} / \mathrm{min}$. Three TOF spectra per second were acquired in MS1 mode from 100 to 1,700 m/z. The nitrogen temperature was set to $320^{\circ} \mathrm{C}$, with a flow of $8 \mathrm{~L} / \mathrm{min}$ and a nebulizer pressure of $35 \mathrm{psig}$. The voltages were set as follows: Nozzle voltage $1000 \mathrm{~V}$, capillary voltage $3500 \mathrm{~V}$, fragmentor $175 \mathrm{~V}$ and skimmer 65 V. Agilent MassHunter Workstation software was used for data processing.

\subsubsection{Quantification of fungal biomass}

In order to normalize the degradation efficiency by fungal biomass, two quantification methods were used in this study. Fungal mycelia after supernatants were lyophilized. Lyophilized mycelia were disintegrated by grinding with $500 \mu \mathrm{l}$ of lysis buffer containing $50 \mathrm{mM}$ Tris buffer, $\mathrm{pH} 8.0$ and $2 \mathrm{mM}$ dithiothreitol. The grinding was performed in reciprocal mill MM 4000 (Retsch, Haan, Germany) in $2 \mathrm{ml}$ Eppendorf tubes with four $2 \mathrm{~mm}$ stainless steel beads (Kugel-Winnie, Germany) at 30 beads/sec for $1 \mathrm{~min}$.

Fungal biomass was measured based on its turbidity. After grinding, $200 \mu \mathrm{l}$ of the blended mycelia suspension was immediately placed into the well of a 96-well plate. A series of VL43 mycelial samples weighing $2 \mathrm{mg}, 1.5 \mathrm{mg}, 1 \mathrm{mg}, 0.5 \mathrm{mg}, 0.1 \mathrm{mg}$ and $0.02 \mathrm{mg}$ was used as standards. The OD of the samples and standards were measured at $340 \mathrm{~nm}$ by Epoch Microplate Spectrophotometer (BioTek, Bad Friedrichshall, Germany). The weight of the mycelia sample was calculated based on standard curves. 
We also quantified fungal biomass based on protein content by Bradford method. The homogenates of fungal cultures were centrifuged for $10 \mathrm{~min}$ at $12,000 \mathrm{rpm}$. $200 \mu \mathrm{l}$ of the supernatants were added into the well of 96-well microtiter plate containing $50 \mu$ of Bradford reagent (Roth, Karlsruhe, Germany). Standards consisted of $100 \mu \mathrm{g} / \mathrm{ml}, 80 \mu \mathrm{g} / \mathrm{ml}, 60 \mu \mathrm{g} / \mathrm{ml}$, $50 \mu \mathrm{g} / \mathrm{ml}, 40 \mu \mathrm{g} / \mathrm{ml}, 30 \mu \mathrm{g} / \mathrm{ml}, 20 \mu \mathrm{g} / \mathrm{ml}, 15 \mu \mathrm{g} / \mathrm{ml}, 10 \mu \mathrm{g} / \mathrm{ml}, 5 \mu \mathrm{g} / \mathrm{ml}, 1 \mu \mathrm{g} / \mathrm{ml}$ and $0 \mu \mathrm{g} / \mathrm{ml}$ of bovine serum albumin solutions.

\subsection{Results}

\subsubsection{Phytoalexins in xylem sap of oilseed rape plants infected with $V$. longisporum}

Twenty to $100 \mu \mathrm{l}$ of xylem sap were collected from each of 8 infected plants and mock-infected control plants with the help of a Scholander pressure chamber. The samples were analyzed by HPLC-MS-QTOF in a positive ionization mode (Table 2). Four phytoalexins were identified: brassilexin, cyclobrassinin, rutalexin and spirobrassinin (Figs. 3 and 4). None of those phytoalexins were detectable in healthy control plants.

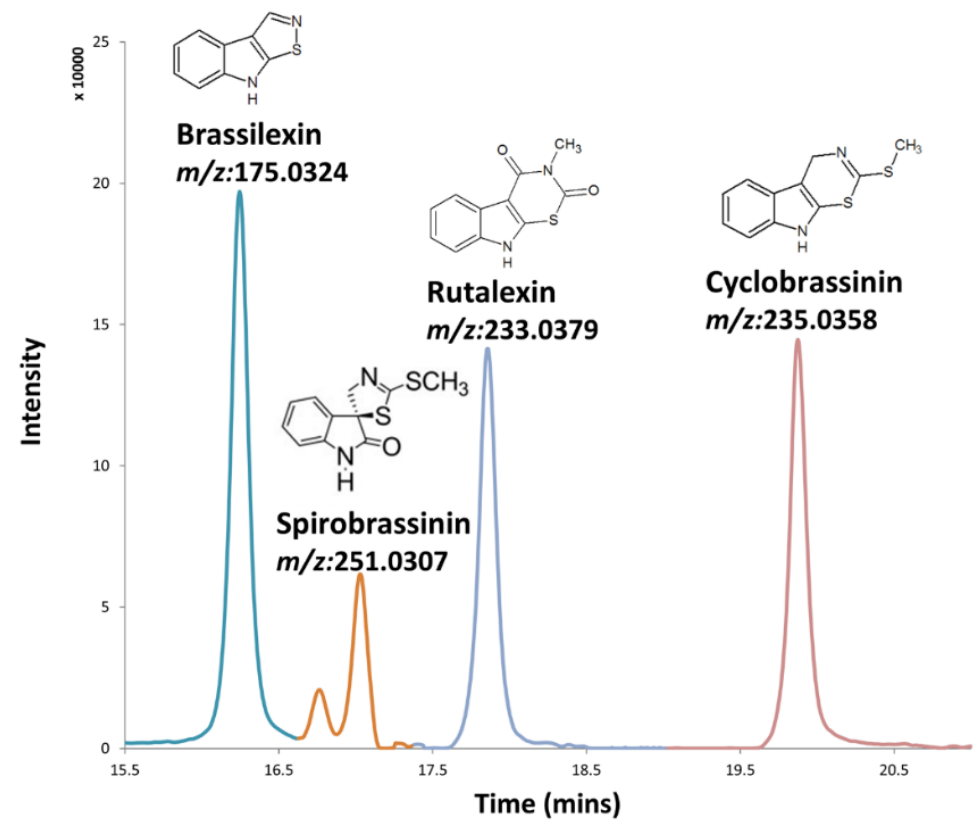

Fig. 3 V. longisporum VL43 infection caused accumulation of phytoalexins in xylem sap of oilseed rape. Xylem sap was collected from an individual 42 dpi rapid-cycling oilseed rape plant with a Scholander pressure chamber. The four induced phytoalexins were identified by HPLC-MS-QTOF (positive mode) based on their mass $(\mathrm{m} / \mathrm{z})$ using commercial (brassinin and brassilexin) and synthesized (cyclobrassinin, spirobrassinin and rutalexin) standards. All four phytoalexins were undetectable in xylem sap of healthy control plants. 
Table 2. Retention time and $m / z$ of phytoalexins detected by HPLC-MS-QTOF

\begin{tabular}{llll}
\hline Phytoalexin & Formula & Retention time $(\mathbf{m i n})$ & $\mathbf{m} / \mathbf{z}$ \\
\hline Brassilexin & $\mathrm{C}_{9} \mathrm{H}_{6} \mathrm{~N}_{2} \mathrm{~S}$ & 15.7 & $(\mathrm{M}+\mathrm{H})^{+} 175.0324$ \\
Spirobrassinin & $\mathrm{C}_{11} \mathrm{H}_{10} \mathrm{~N}_{2} \mathrm{OS}_{2}$ & 16.5 & $(\mathrm{M}+\mathrm{H})^{+} 251.0307$ \\
Rutalexin & $\mathrm{C}_{11} \mathrm{H}_{8} \mathrm{~N}_{2} \mathrm{O}_{2} \mathrm{~S}$ & 17.3 & $(\mathrm{M}+\mathrm{H})^{+} 233.0379$ \\
Cyclobrassinin & $\mathrm{C}_{11} \mathrm{H}_{10} \mathrm{~N}_{2} \mathrm{OS}_{2}$ & 19.3 & $(\mathrm{M}+\mathrm{H})^{+} 235.0358$ \\
Brassinin & $\mathrm{C}_{11} \mathrm{H}_{12} \mathrm{~N}_{2} \mathrm{~S}_{2}$ & 16.9 & $(\mathrm{M}+\mathrm{Na})^{+} 259.0334$ \\
1-methoxybrassinin & $\mathrm{C}_{12} \mathrm{H}_{14} \mathrm{~N}_{2} \mathrm{OS}$ & $(\mathrm{M}+\mathrm{H})^{+} 267.0620$ \\
\hline
\end{tabular}

- No standard available

Brassinin was not induced by the virulent strain V. longisporum VL43 to a detectable level. Avirulent strains VL1191 and VL1199 also elicited brassilexin, cyclobrassinin, spirobrassinin and rutalexin synthesis while brassinin was also not induced (data not shown). Replicate experiments showed essentially the same results.

In addition to the five phytoalexins mentioned before, HPLC-MS data were searched for further phytoalexins known from crucifers according to their high-resolution $\mathrm{m} / \mathrm{z}$. The signals were undetectable or very low, including 1-methoxybrassinin which was reported from oilseed rape before. Only signals corresponding to cyclobrassinin sulfoxide were found but due to the lack of a standard, the identity of the analyte could not be verified. Therefore, only the induction of brassilexin, cyclobrassinin, spirobrassinin and rutalexin by $V$. longisporum infection could be confirmed.

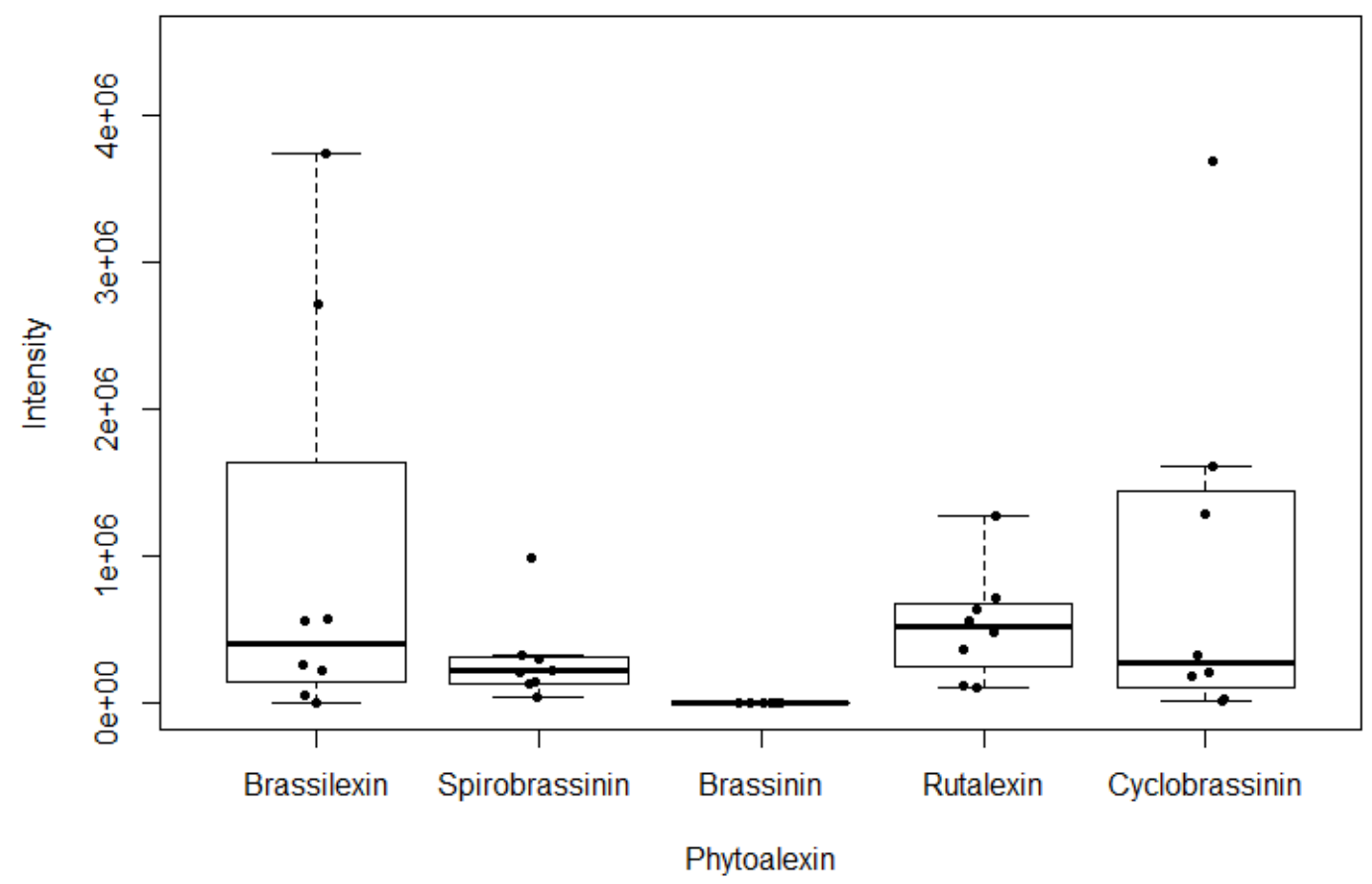


Fig. 4 Accumulation of phytoalexins in xylem sap of oilseed rape plants infected with $\mathbf{V}$. longisporum . Boxes represent the $75^{\text {th }}$ percentile median and the $25^{\text {th }}$ percentile. Eight replicates of both infected and healthy plants were analyzed.

\subsubsection{Elicitation of phytoalexins in $B$. napus by $\mathrm{CuSO}_{4}$}

All four phytoalexins induced by $V$. longisporum infection were also elicited by $\mathrm{CuSO}_{4}$. $\mathrm{Plants}$ infected with $V$. longisporum were examined in the same experiment for a comparison (Fig. 5).

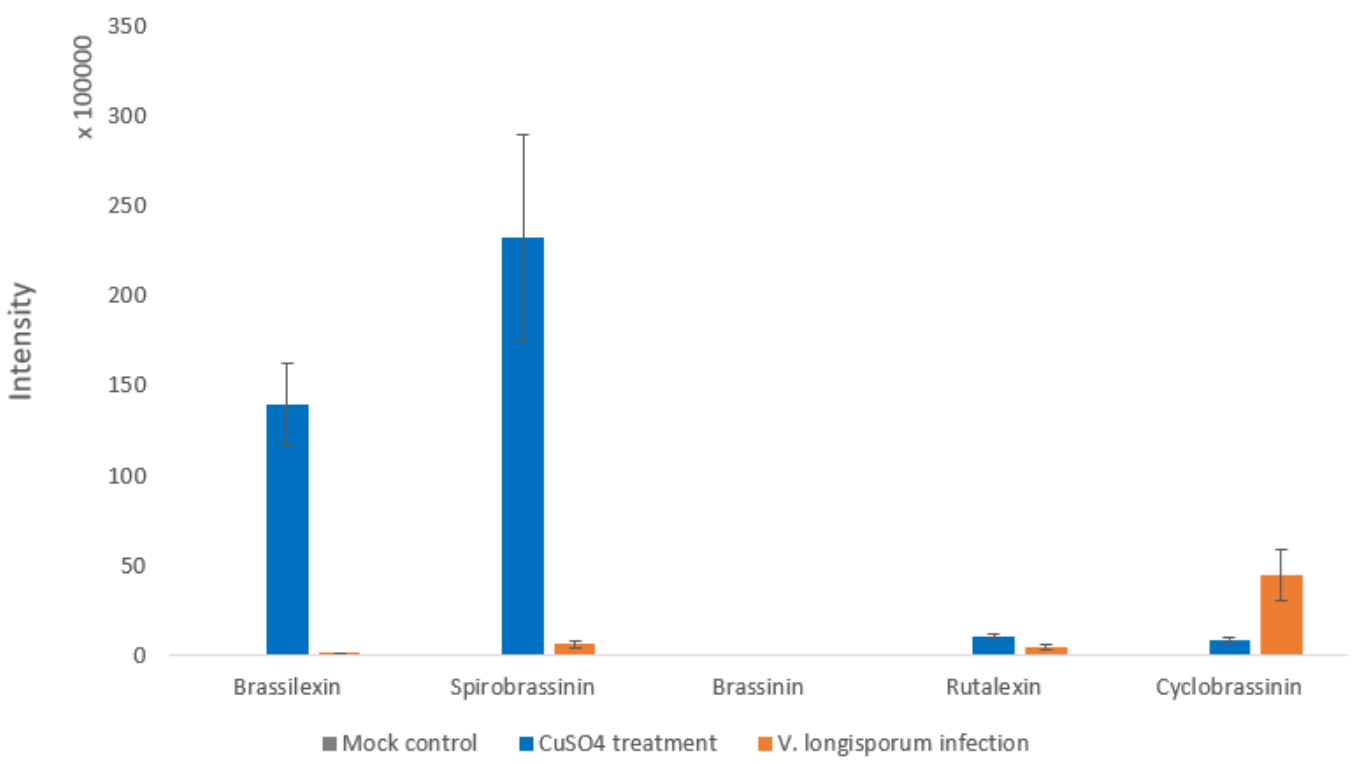

Fig. 5 Induction of phytoalexin synthesis in oilseed rape plants by Verticillium longisporum infection and $\mathrm{CuSO}_{4}$ treatment. Phytoalexin levels were determined in extracts of entire plants without roots. All five tested phytoalexins were non-detectable in control plants mock-inoculated with sterile tap water. Blue bars represent phytoalexins produced in plants sprayed with $10 \mathrm{mM} \mathrm{CuSO}_{4}$ solution. Orange bars are the samples extracted from plants infected with $V$. longisporum (VL43). Brassinin was not detected in any sample. Eight replicates were used.

The plants accumulated around 700 times and 40 times more brassilexin and spirobrassinin, respectively, after $\mathrm{CuSO}_{4}$ treatment as compared with V. longisporum infection. Cyclobrassinin was the only phytoalexin that accumulated in higher amounts after $V$. longisporum infection than after $\mathrm{CuSO}_{4}$ treatment. 


\subsubsection{Brassilexin degradation in fungal cultures}

Figure 6 illustrates how brassilexin was degraded in cultures of various fungi after 6 hours incubation. The cultures included Verticillium species, other cruciferous pathogens and fungi that are not able to infect crucifers. The fastest degradation of brassilexin was detected in Verticillium cultures; isolates virulent on oilseed rape metabolized phytoalexins slightly more efficiently than less virulent isolates. However, although $V$. dahliae does not infect crucifers in nature, V. dahliae showed a degradation efficiency comparable to virulent $V$. longisporum strains. This indicates that brassilexin degradation is not a specific capability related to the adaptation of $V$. longisporum to crucifers. Interestingly, Verticillium species, including $V$. longisporum, V. dahliae and V. alboatrum, degraded brassilexin more efficiently than other crucifer pathogens, such as S. sclerotiorum, R. solani, and L. maculans.

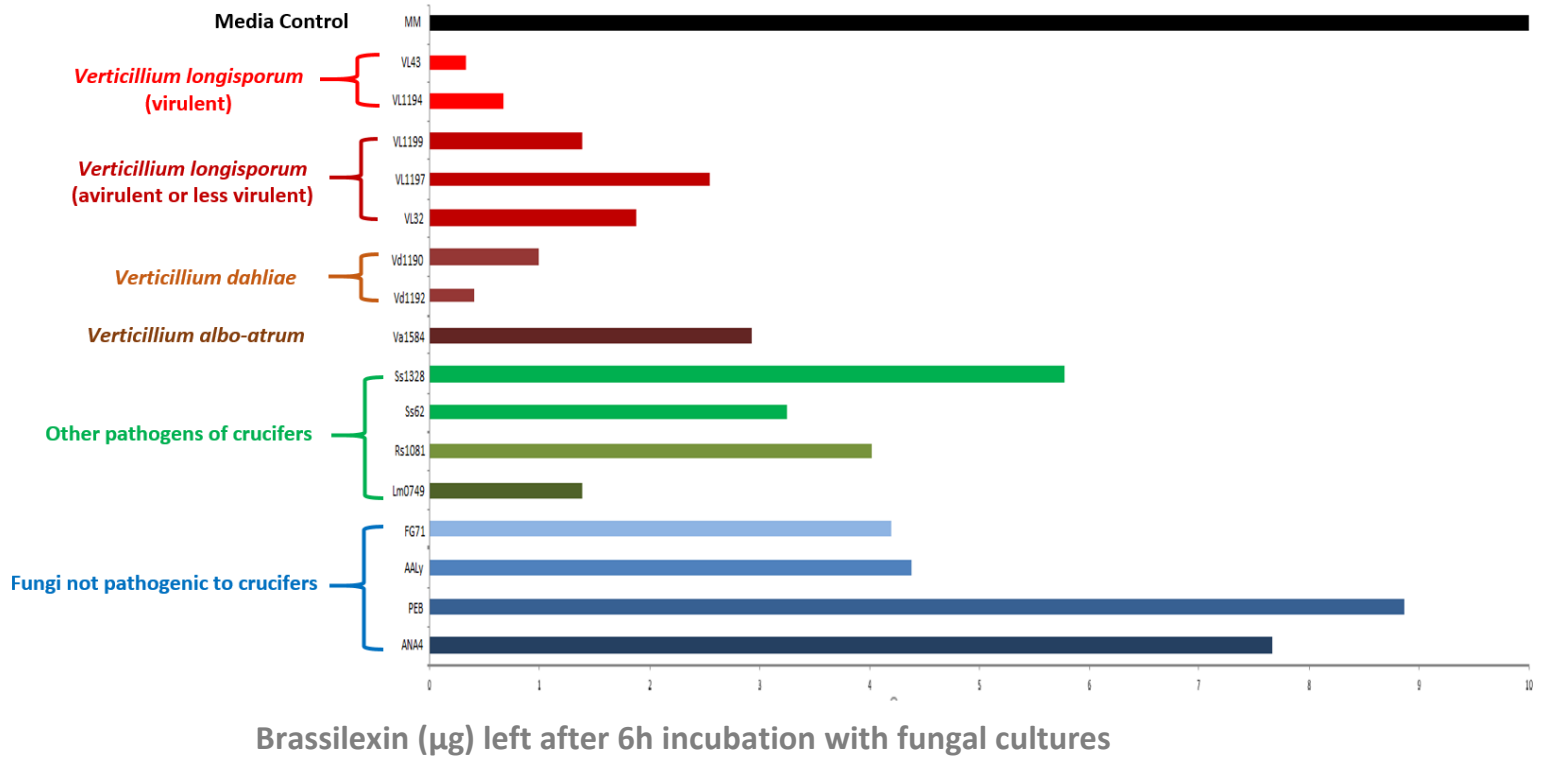

Fig. 6 Brassilexin degradation by fungi. $10 \mu \mathrm{g}$ of brassilexin were incubated in $500 \mu 1$ culture medium with various fungi for $6 \mathrm{~h}$ and the supernatants were analyzed by HPLC-DAD. The length of each bar represents the remaining amount of brassilexin. Species representing other pathogens of crucifers were Sclerotinia sclerotiorum (Ss1328 and Ss62), Rhizoctonia solani (Rs1081), Leptosphaeria maculans (Lm0749). Species representing fungi not pathogenic to crucifrs were Fusarium graminearum (FG71), Alternaria alternata (AAy), Penicillium expan (PEB) and Aspergillus nidulans (ANA4).

In order to clarify whether brassilexin degradation efficiency was related to the virulence of pathogenic species, we further analyzed differences between Verticillium species that are either virulent or avirulent to oilseed rape in short-time incubation. We reasoned that slow degradation 
it would not benefit the pathogen much. Therefore, a short (30 min), rather than long $(72 \mathrm{~h}$ ) incubation time was used. Figure 7 shows the degradation abilities of virulent and avirulent Verticillium strains after $30 \mathrm{~min}$. In all Verticillium cultures, $10 \%$ to $30 \%$ of brassilexin were degraded after 30 min incubation regardless of whether the strain was virulent on oilseed rape. One of $V$. dahliae strains was the fastest degrader. The difference in degradation ability among Verticillium species increased after $6 \mathrm{~h}$ incubation (Fig. 8). Two virulent $V$. longisporum strains degraded more brassilexin than the avirulent isolates, but V. dahliae, and even V. albo-atrum, showed similar degradation abilities as the virulent strains.. Based on these results, brassilexin degradation ability does not seem to affect pathogenicity and virulence of Verticillium spp. on oilseed rape.

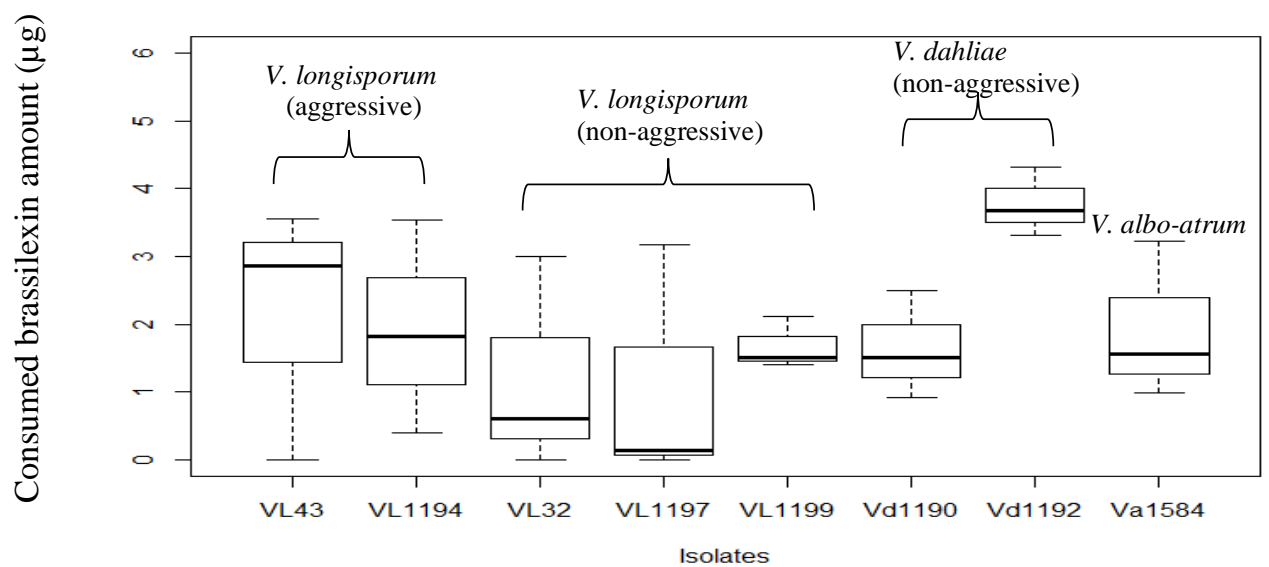

Fig. 7 Degradation of brassilexin by Verticillium spp. in 30 min. Fungal biomass was quantified based on turbidity. The initial amount of brassilexin was $10 \mu \mathrm{g}$. Boxes represent the decreased amount of brassilexin in each fungal cultures with $75^{\text {th }}$ percentile median and the $25^{\text {th }}$ percentile. Triplicates were performed for each strain. 


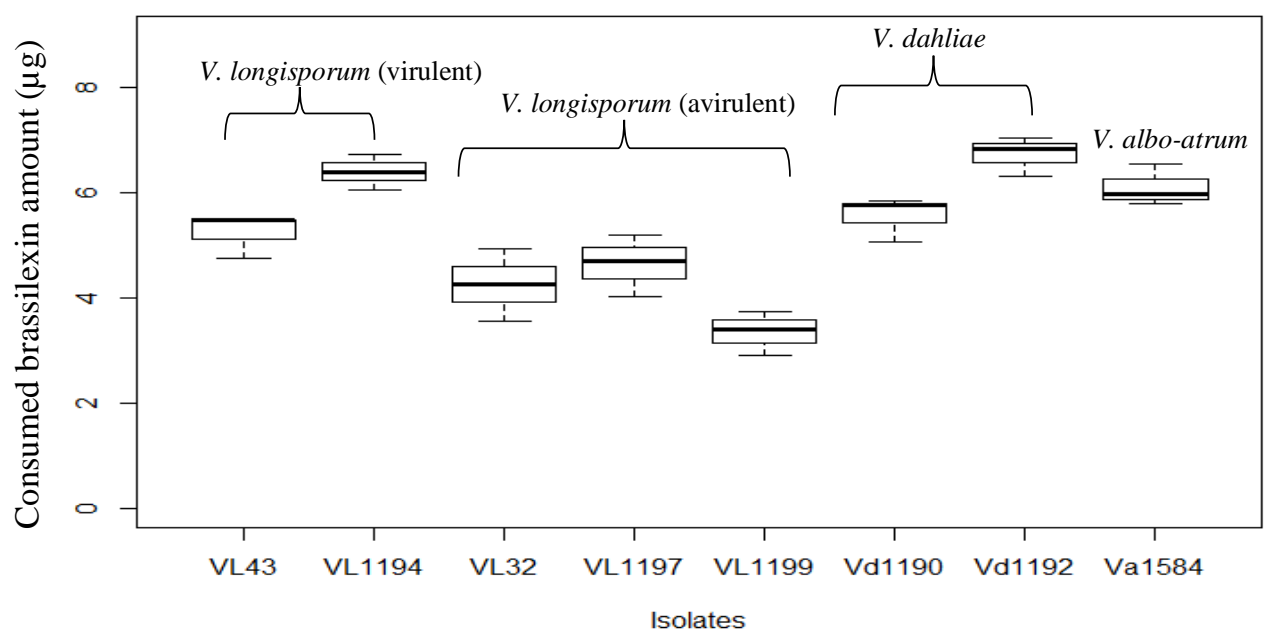

Fig. 8 Degradation of brassilexin by Verticillium spp. in $6 \mathrm{~h}$. Fungal biomass was quantified based on turbidity. The initial amount of brassilexin was $10 \mu \mathrm{g}$. Boxes show 75th percentile, median, and the 25th percentile. The assays were carried out in triplicates.

For the analysis of the degradation products of brassilexin by HPLC-MS, the incubation time was further extended. Twenty $\mu \mathrm{g}$ brassilexin per $\mathrm{ml}$ were completely degraded in less than $20 \mathrm{~h}$ by most of Verticillium strains (Fig. 9). The known fungal metabolites of brassilexin are shown in Figure 12 are 3-(amino)methylenindoline-2-thione produced by L. maculans and S. sclerotiorum; 3-formylindolyl-2-sulfonic acid produced by L. maculans; brassicanal A produced by S. sclerotiorum; and 1-ß-D-glucopyranosylbrassilexin produced by S. sclerotiorum (Pedras and Suchy 2005; Fradin and Thomma 2006; Pedras and Hossain 2006).

In products of the incubation of brassilexin with $V$. longisporum, a new HPLC-DAD peak at a retention time of $3.8 \mathrm{~min}$ was found. The area of the peak grew parallel to the decrease of the peak of brassilexin in time (data not shown). Based on HPLC-MS-QTOF data, the compound was predicted to be 3-(amino)methylenindoline-2-thione. It was detectable already 5 min after incubation of brassilexin with fungal cultures. The peak area decreased after $48 \mathrm{~h}$ incubation, indicating that 3-(amino)methylenindoline-2-thione was an intermediate rather than the final degradation product (Fig. 11). 3-(amino)methylenindoline-2-thione was described as an intermediate of brassilexin transformation by well-studied pathogens, such as L. maculans and $S$. sclerotiorum. Interestingly, our experiments has shown that 3-(amino)methylenindoline-2-thione was also produced by fungi that were not able to infect crucifers, including Alternaria alternata, 
Aspergillus nidulans, and Fusarium graminearum. Moreover, the amount produced by nonpathogens of oilseed rape was comparable to the amounts produced by pathogens. The metabolite was not found in media without any fungus, showing that it was not generate by spontaneous degradation.

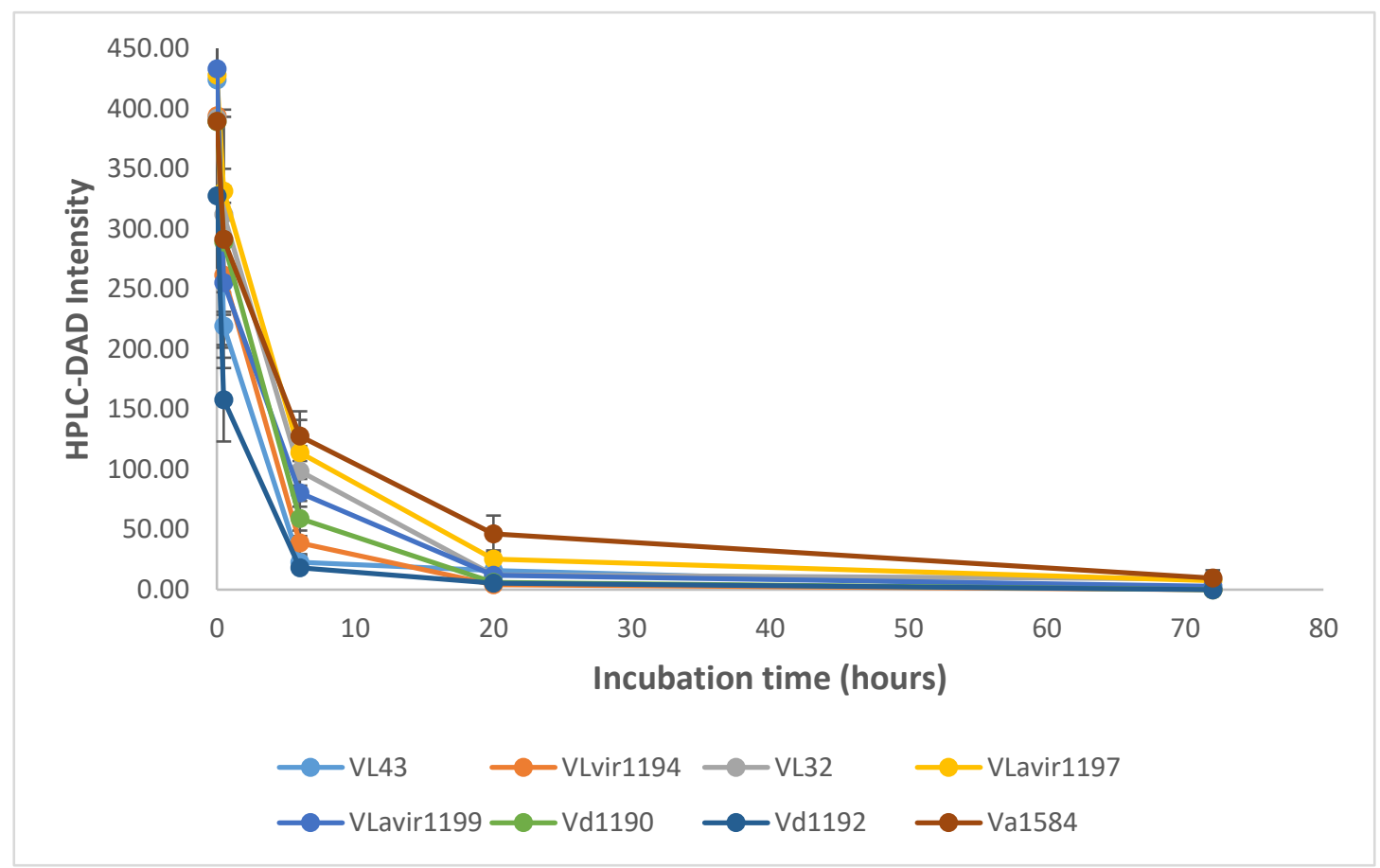

Fig. 9 Degradation progress curves of brassilexin by Verticillium spp. Samples were analyzed by HPLC-DAD. Points are the averages of tests conducted in triplicate \pm standard error. Each line color represents a Veticillium strain listed in Table 1.

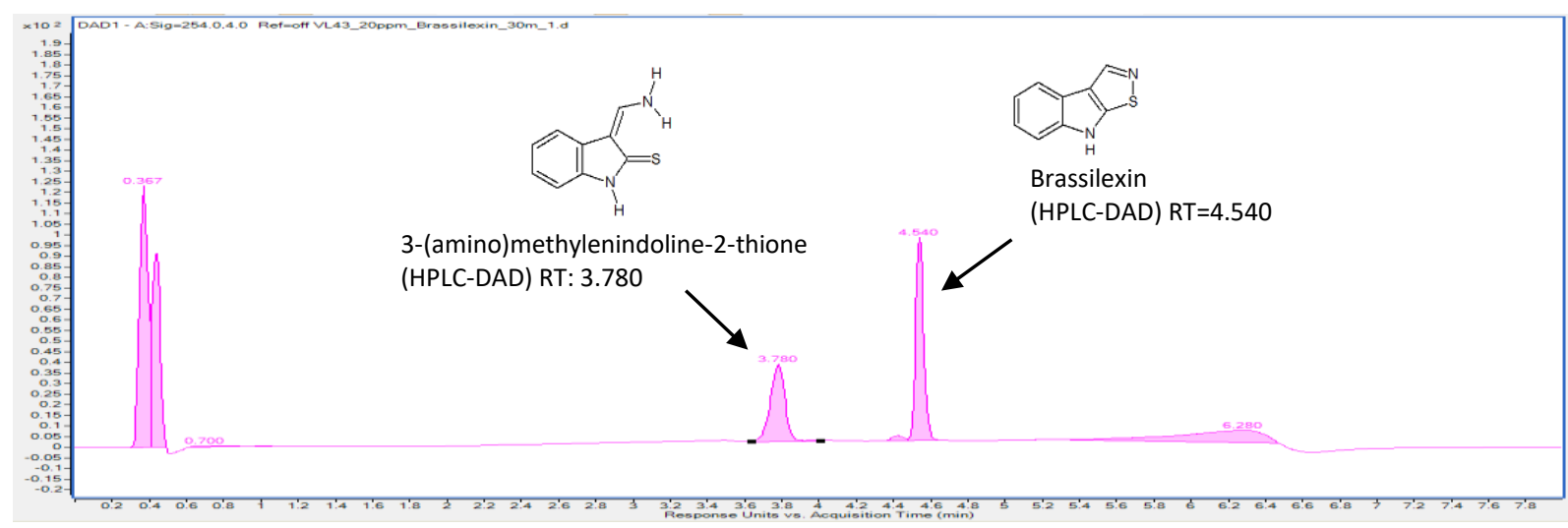

Fig. 10 A HPLC peak ( $R$ T=3.8 min) corresponding to a new metabolite was detected after 30 min incubation of $20 \mu \mathrm{g} / \mathrm{ml}$ brassilexin with $V$. longisporum VL43. The detection was by HPLC-DAD. The peak at $\mathrm{RT}=4.5 \mathrm{~min}$ is brassilexin. 


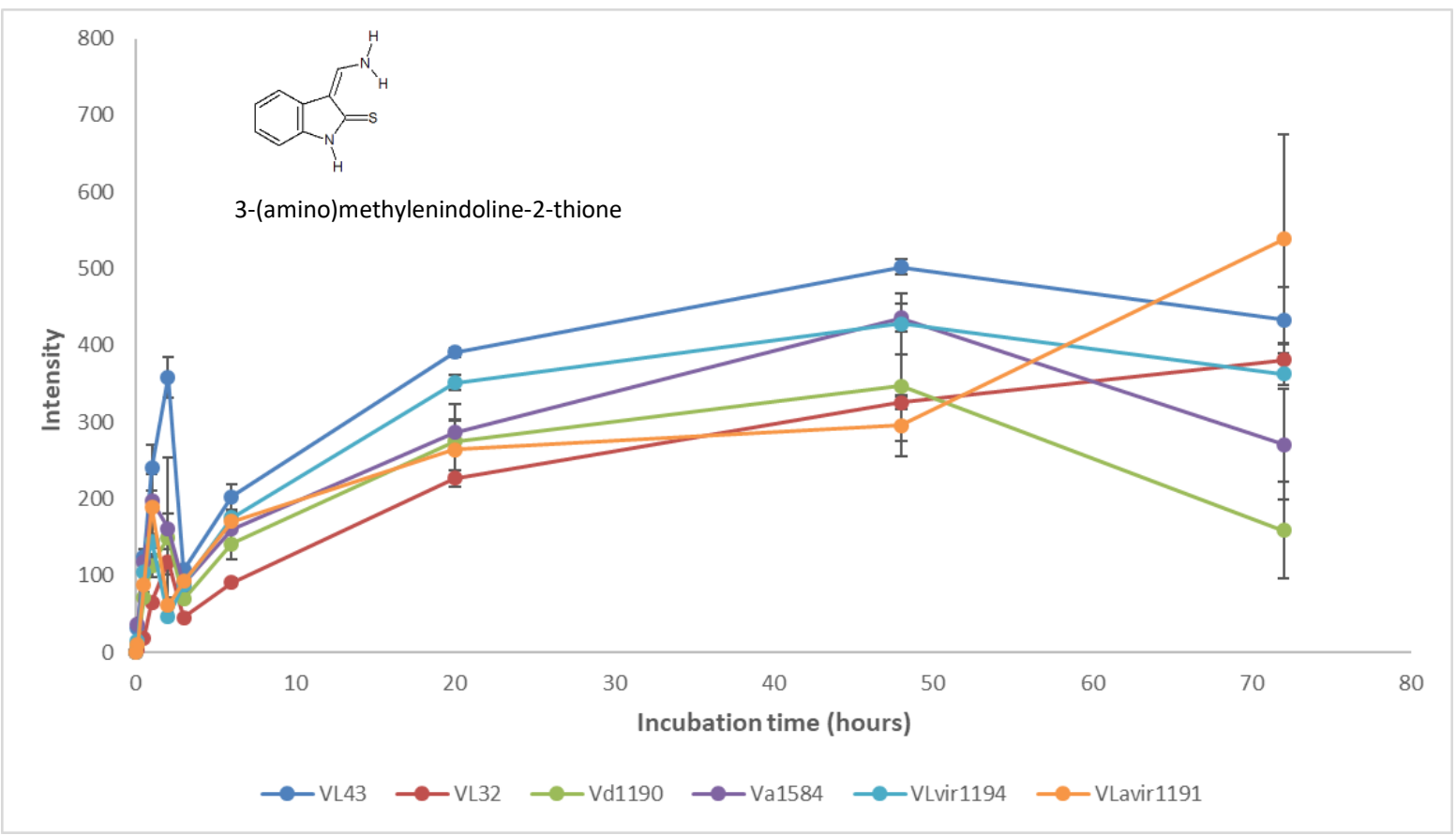

Fig. 11. Production of 3-(amino)methylenindoline-2-thione in cultures of various Verticillium strains incubated with brassilexin. Samples were analyzed by HPLC-DAD. Averages of triplicate tests \pm standard error are shown. Each line color represents a different Verticillium strain (Table 1).

In addition to 3-(amino)methylenindoline-2-thione, brassicanal $\mathrm{A}$ was also detected in Verticillium cultures incubated with brassilexin, but the same metabolite was found in all other fungal cultures, too, and even in minimum media without fungi. Brassicanal A was described as the product in a minor pathway of brassilexin transformation by S. sclerotiorum. After 72 hours incubation, brassicanal A amounts in Verticllium spp. cultures were 10 times lower than in S. sclerotiorum, but more than 1,000 times higher than in controls without fungi (Fig. 12). Therefore, Verticillium species may also produce brassicanal A as a degradation product, but most likely in a minor pathway.

Glycosylation is not a common reaction in fungal biotransformations. We found 1- $\beta$-Dglucopyranosylbrassilexin only after incubation of brassilexins with S. sclerotiorum. Both $S$. sclerotiorum strains but none of the other fungal species tested produced 1-B-Dglucopyranosylbrassilexin in a detectable level (Fig. 12).

However, not all known brassilexin degradation product could be detected in the investigated fungal cultures. 3-Formylindolyl-2-sulfonic acid was described as a transformation product of 
L. maculans. We have not found this metabolite in any fungal culture incubated with brasilexin, even not in L. maculans. 


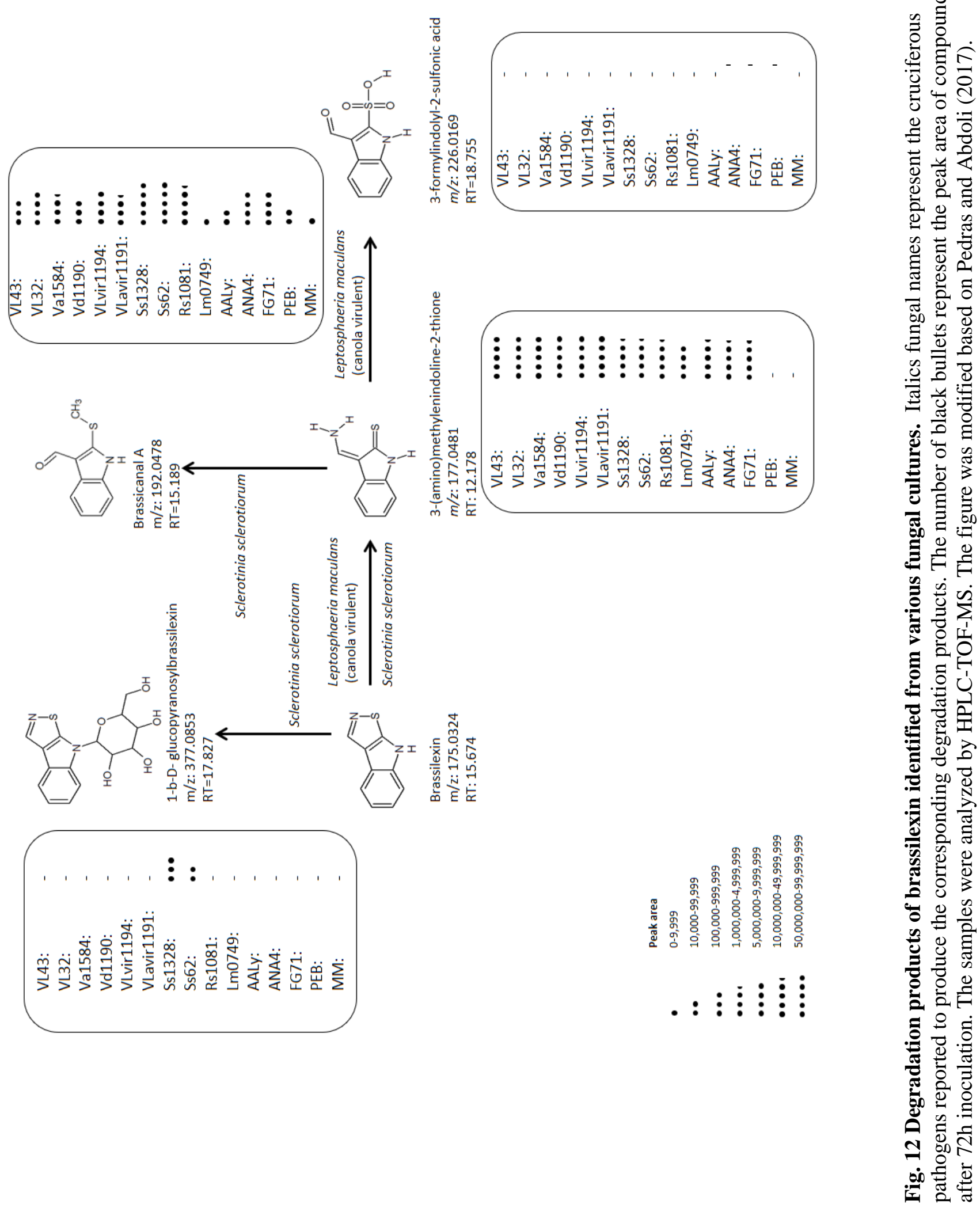




\subsubsection{Brassinin degradation by fungal cultures}

No apparent degradation of brassinin was detected in cultures of Verticillium species after $20 \mathrm{~h}$ incubation (Fig. 13). Although some brassinin was degraded in Verticillium cultures after $72 \mathrm{~h}$, the degraded amount was similar as in cultures of fungi that are not able to infect crucifers, and much lower than in cultures of other cruciferous pathogens, such as S. sclerotiorum, $R$. solani and L. maculans (Fig. 14).

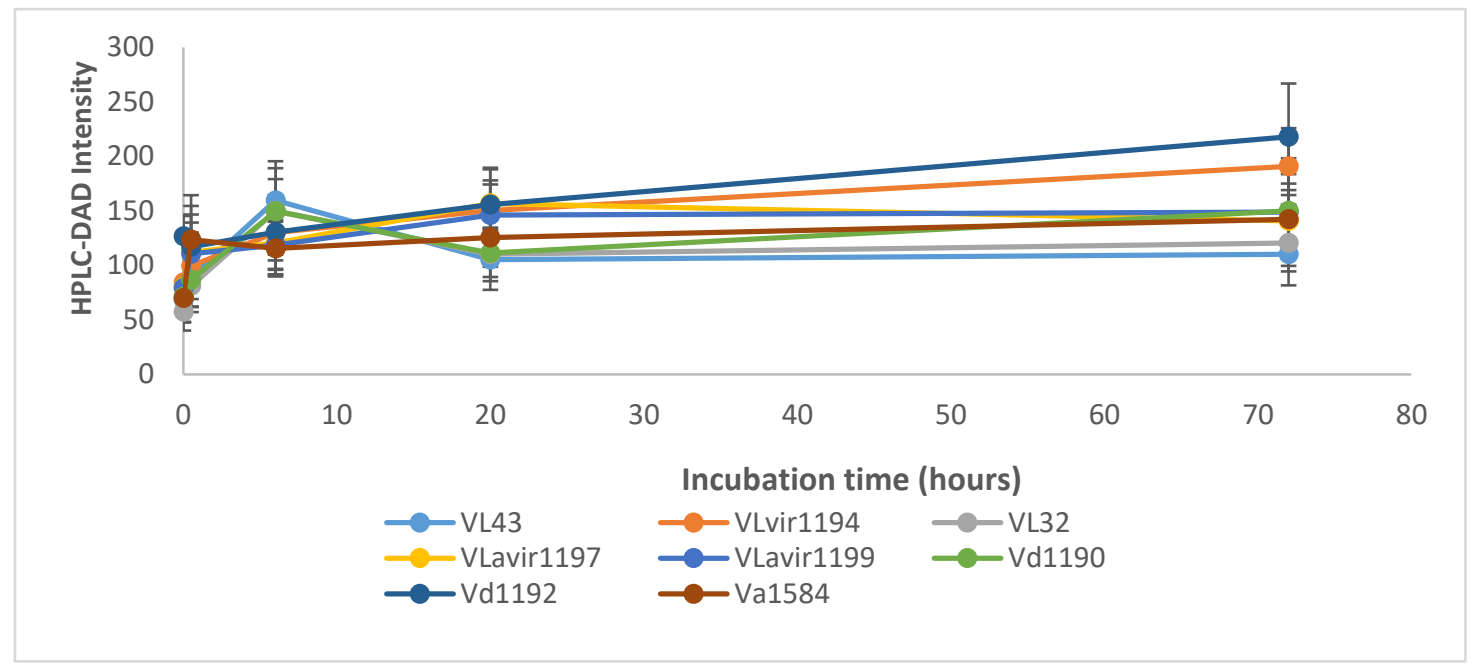

Fig. 13 Degradation progress curves of brassinin in various Verticillium cultures. Samples were analyzed by HPLC-DAD. Mean of tests conducted in triplicate \pm standard errors are shown. The Verticillium strains used are listed in Table 1.

It seems that brassinin cannot be degraded by Verticillium species. Figure 15 shows two degradation products detected in Verticillium cultures. However, indole-3-carboxaldehyde was also found in control medium that was free of fungal mycelium. It may therefore be a product of spontaneous breakdown of brassinin.

Indole-3-carboxylic acid was described as degradation product of brassinin by a variety of fungi, including L. maculans (canola virulent), L. maculans (brown mustard virulent), A. brassicicola and B. cinerea (Pedras et al. 2009a; Soledade et al. 1993; Pedras et al. 2011a). All these fungal species except $S$. sclerotiorum were included in this study. The amounts of indole-3-carboxylic acid detected in Verticillium cultures were similar to those of cultures of fungi which cannot infect crucifers, but almost 100 times lower than in cultures L. maculans, which is known to degrade brassinin to this compound. 


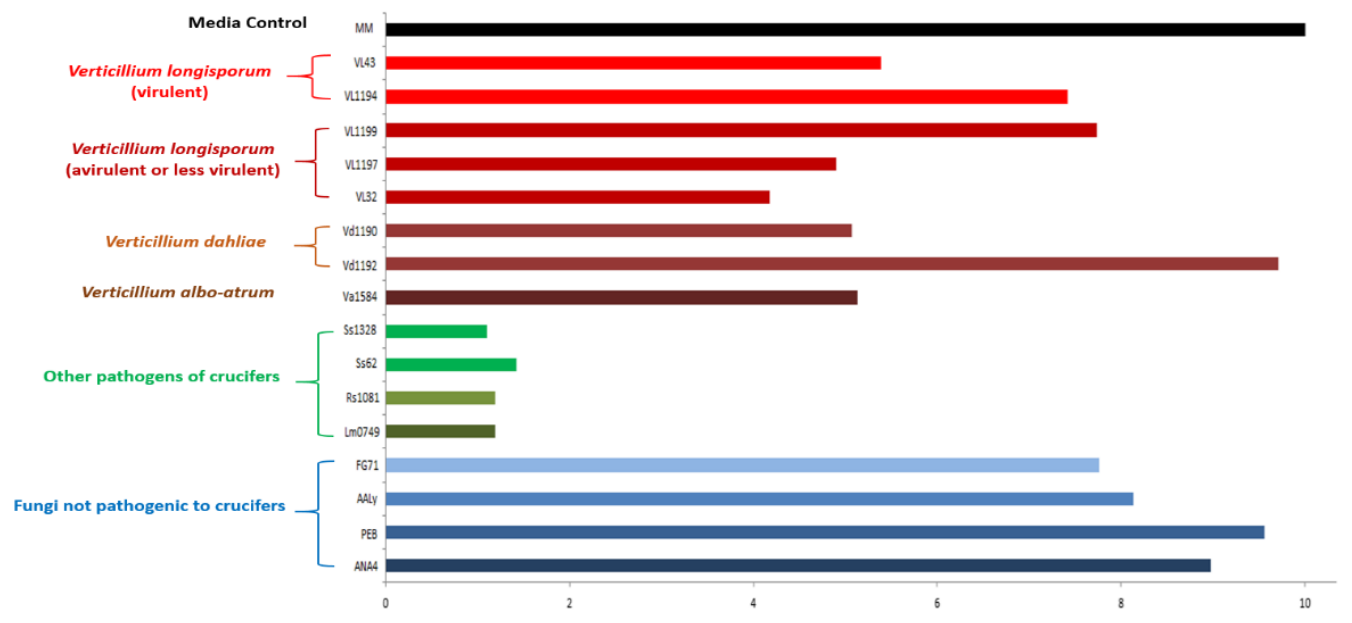

Detectable amount $(\mu \mathrm{g})$ of brassinin in fungal culture after $72 \mathrm{~h}$ incubation

Fig. 14 Brassinin degradation after $72 \mathrm{~h}$ incubation with various fungi. Samples were analyzed by HPLC-DAD. The length of each bar represents the detectable amount of brassinin (RT=4.8 min) in fungal culture. Fungal strains are listed in Table 1. The initial amount of brassinin was $10 \mu \mathrm{g}$. Species representing other pathogens of crucifers were Sclerotinia sclerotiorum (Ss1328 and Ss62), Rhizoctonia solani (Rs1081), Leptosphaeria maculans (Lm0749). Species representing fungi not pathogenic to crucifrs were Fusarium graminearum (FG71), Alternaria alternata (AAy), Penicillium expan (PEB) and Aspergillus nidulans (ANA4). 

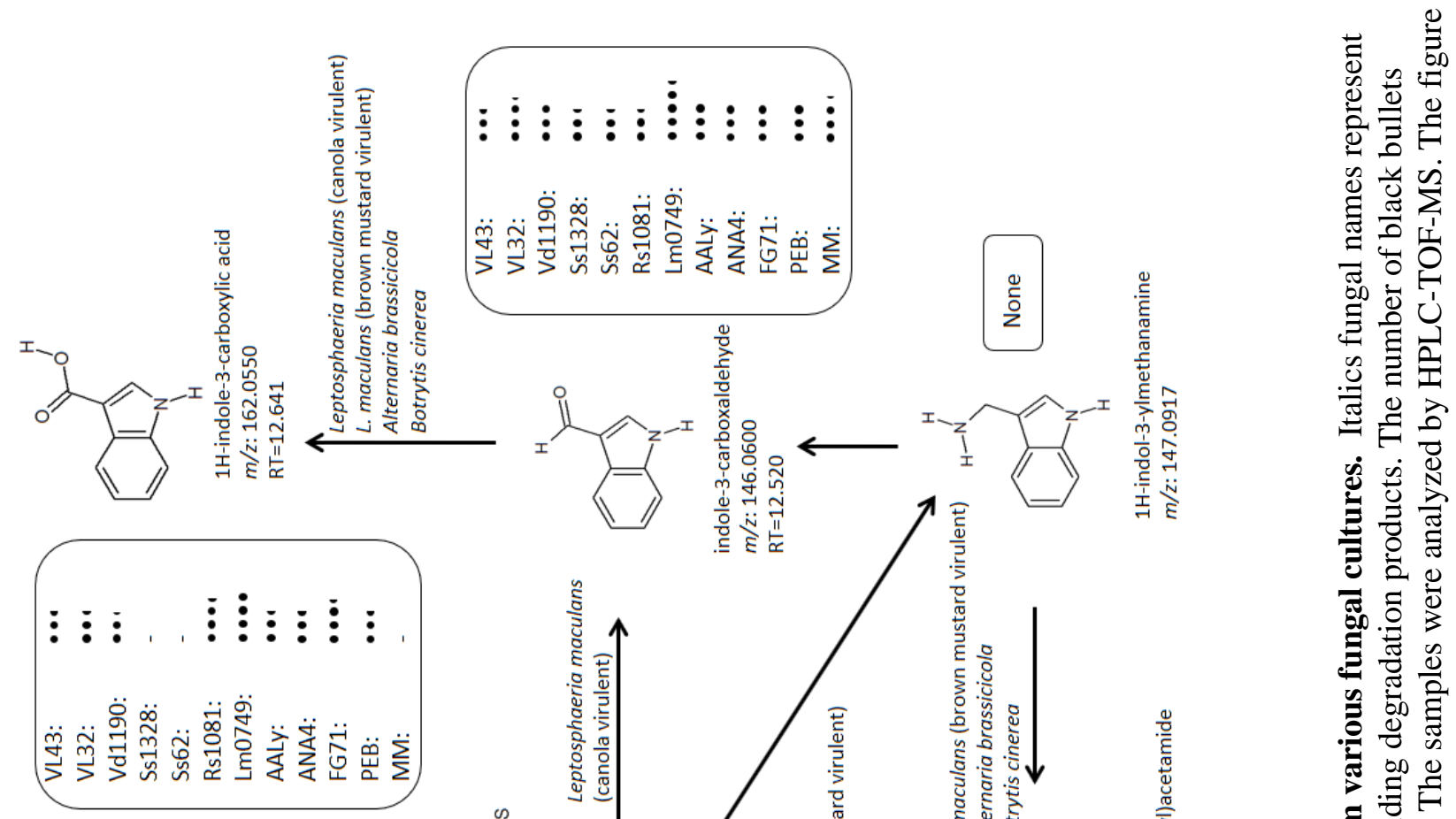

के चै

壱壱

สㅇํㅇ

离

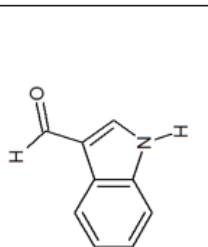

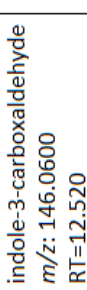
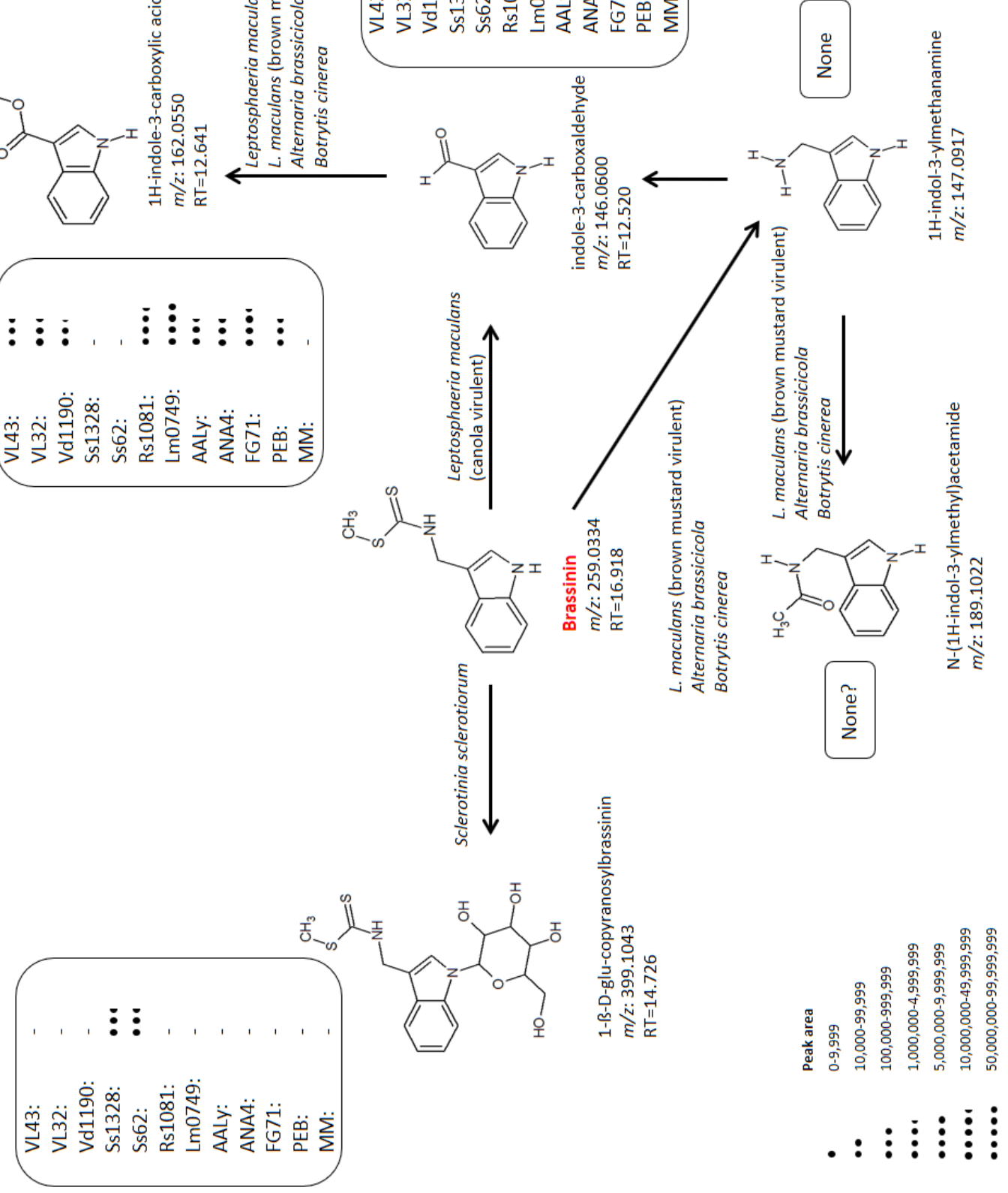

氜

跑

递离

ह

대요

恶 胥

影

50

क्ष

$\Xi \Xi E$

일

Dृ

ठ ठ ठ

로

웡유

.

它

웡 0 过

뭉

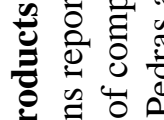

司

。ี

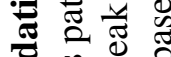

क्षे

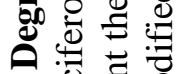

늘 ठ⿹

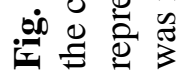




\subsubsection{Cyclobrassinin degradation in fungal cultures}

Unexpectedly, cyclobrassinin $(\mathrm{RT}=5.3 \mathrm{~min}$ ) was degraded in pure minimum media without fungi faster than in Verticillium cultures, but slower than in S. sclerotiorum cultures (Fig. 16).

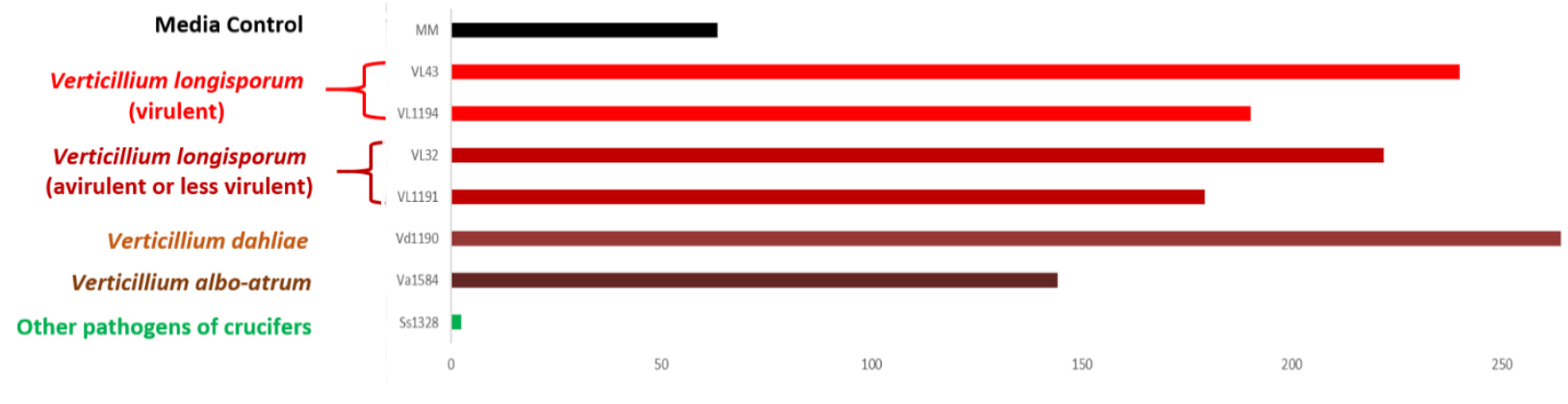

Detectable amount of cyclobrassinin in fungal culture after 20h incubation ( $\mu \mathrm{g})$

Fig. 16 Cyclobrassinin degradation after 20h incubation with various fungi. Samples were analyzed by HPLC-DAD. The length of each bar represents the detectable amount of cyclobrassinin (RT=5.3 min) in fungal culture. Fungal strains are listed in Table 1. The initial amount of cyclobrassinin was $10 \mu \mathrm{g}$. Species representing other pathogens of crucifers were Sclerotinia sclerotiorum (Ss1328 and Ss62), Rhizoctonia solani (Rs1081), Leptosphaeria maculans (Lm0749). Species representing fungi not pathogenic to crucifrs were Fusarium graminearum (FG71), Alternaria alternata (AAy), Penicillium expan (PEB) and Aspergillus nidulans (ANA4).

A new peak (HPLC-TOF-MS RT=16.8 min) appeared in the medium control after 7 days incubation with cyclobrassinin, while the peak (HPLC-TOF-MS RT=19.3 min) representing cyclobrassinin disappeared (Fig. 17). In addition, the new peak was also detected in pure simulated xylem media (SXM) and natural xylem sap extracted from B. napus incubated with cyclobrassinin for 7 days. Therefore, cyclobrassinin is not stable, and can brake down spontaneously into another compound.

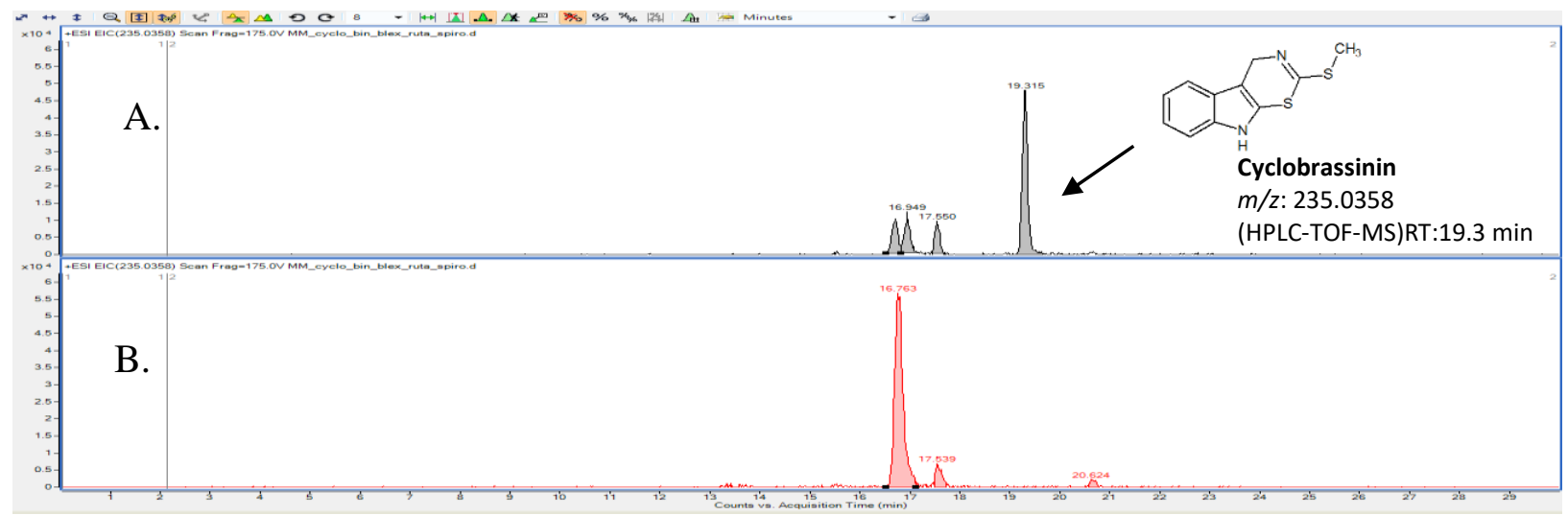

Fig. 17 Cyclobrassinin (A) was completely degraded into another compound (B) without fungi in 7 days (A). Peak (HPLC-TOF-MS RT=19.3 min) extracted from media incubated with $20 \mu \mathrm{g} / \mathrm{ml}$ 
cyclobrassinin for 1 day. (B). Peak (HPLC-TOF-MS RT=16.8) extracted from media incubated with 20 $\mu \mathrm{g} / \mathrm{ml}$ cyclobrassinin inoculated for 7 days .

Figure 18 shows that known degradation products of cyclobrassinin were either non-detectable in cultures of Verticillium species or were also found in media control. This indicates that none of the Verticillium spp. tested can degrade cyclobrassinin into its known fungal degradation products. 


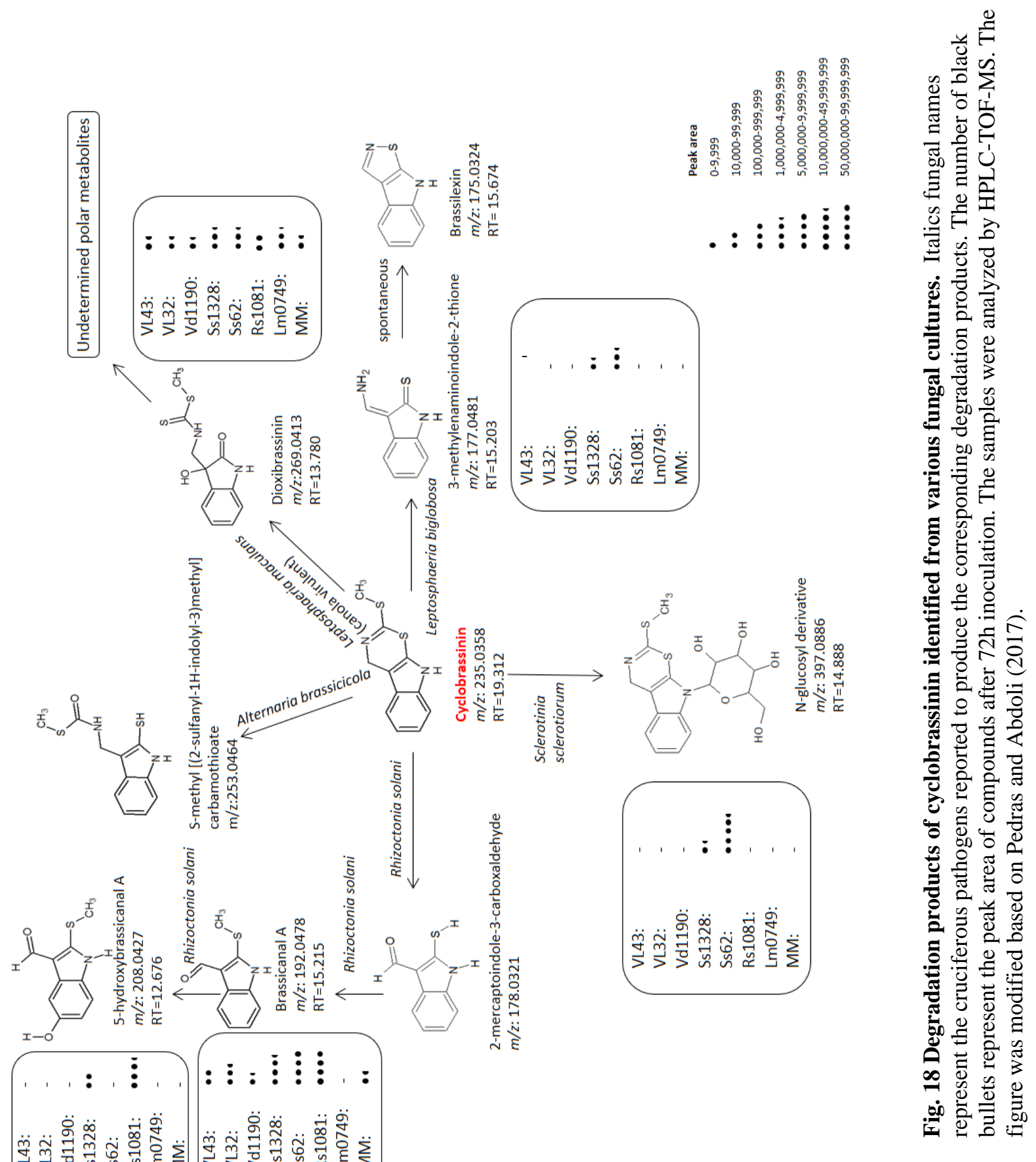




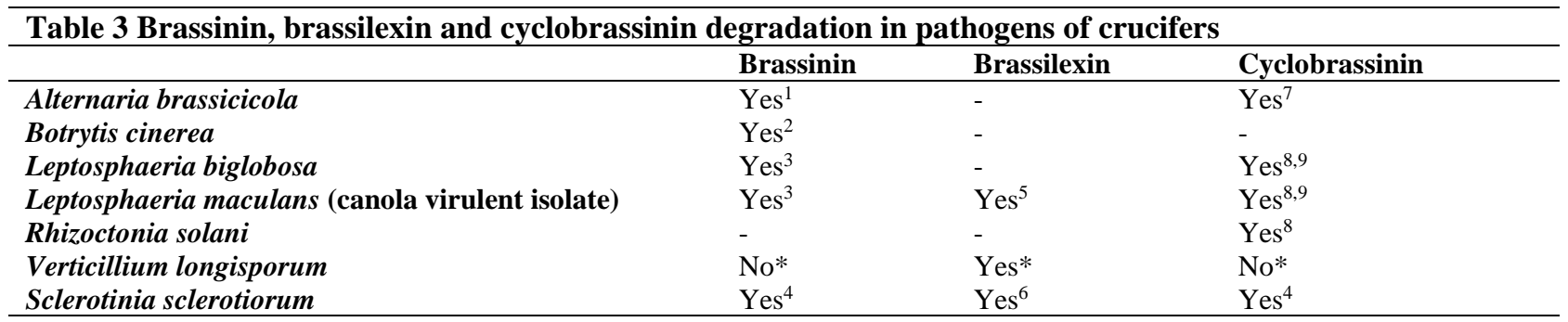

-: no information available

*: this work

References: ${ }^{1}$ : Pedras et al. (2009); ${ }^{2}$ : Pedras et al. (2011): ${ }^{3}$ : Soledade et al. (1993); ${ }^{4}$ Pedras et al. (2004); ${ }^{5}$ : Pedras and Suchy (2005); ${ }^{6}$ : Pedras and Hossain (2006); ${ }^{7}$ : Pedras et al. (2013); ${ }^{8}$ : Pedras and Okanga (1999); ${ }^{9}$ : Pedras (1998);

Table 3 summarizes the results of degradation studies on the three phytoalexins. Most of the fungal pathogens used in the published studies were able to detoxify brassinin. However, the $V$. longisporum strain used in this study failed to degrade brassinin. These strains, though, was able to efficiently degrade brassilexin, which is is a capability that many other fungi lack.

\subsubsection{Brassilexin inhibit germination of $V$. longisporum spores}

Figure 19 shows that brassilexin was active against $V$. longisporum (VL43) germination at a concentration of $20 \mu \mathrm{g} / \mathrm{ml}$. Brassilexin was however not toxic to the fungus when it was added to growing mycelia (Fig. 20).

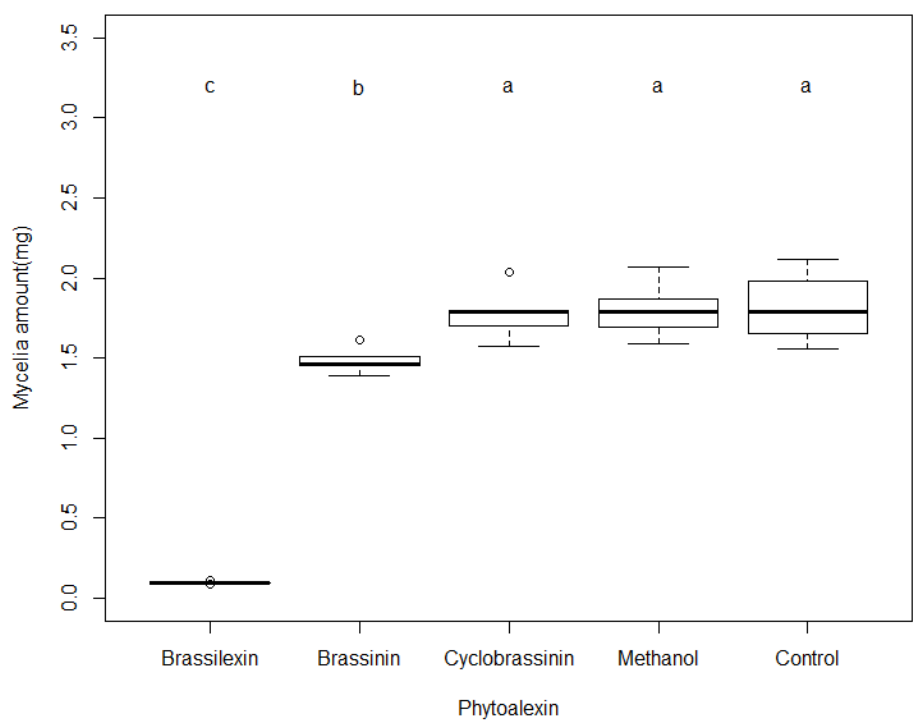

Fig. 19 Toxicity of brassinin, brassilexin and cyclobrassinin tested on spores of VL43. $5 \mu$ of compounds solution was added together with VL43 fungal spore suspension in $500 \mu 1$ minimum media. Final concentration of compounds were $20 \mu \mathrm{g} / \mathrm{ml}$. $5 \mu \mathrm{l}$ of methanol and fungal cultures without any additive were used as two controls, respectively. $5 \mu \mathrm{l}$ of methanol was used for avoiding the fungal growth 
caused by organic solvents. After 22 days incubation at $22^{\circ} \mathrm{C}$ in dark. The fungal mycelia biomass was measured based on turbidity. Boxes represent mycelia biomass with the $75^{\text {th }}$ percentile median and the $25^{\text {th }}$ percentile. Five replicates were performed for each phytoalexin. One-way ANOVA followed by Tukey's HSD test $(\mathrm{p}<0.01)$ is performed for this study.

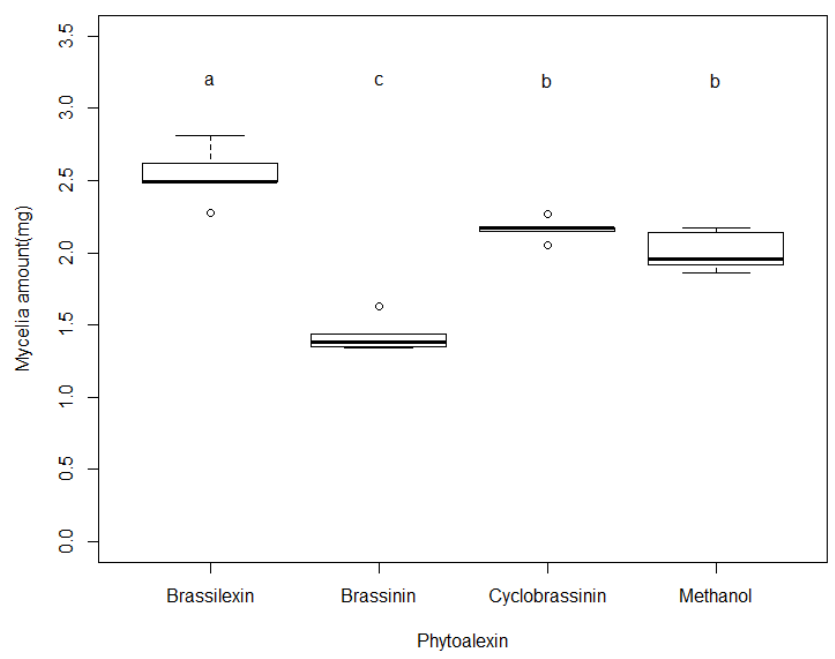

Fig. 20 Toxicity of brassinin, brassilexin and cyclobrassinin to VL43. $10 \mu$ l of compounds solution was added into $500 \mu \mathrm{l}$ of five days old fungal culture in minimum media. Final concentration of compounds are $40 \mu \mathrm{g} / \mathrm{ml}$. $10 \mu \mathrm{l}$ of methanol was used for avoiding the fungal growth caused by organic solvents. After 22 days incubation at $22^{\circ} \mathrm{C}$ in dark. The fungal mycelia biomass was measured based on turbidity. Boxes represent mycelia biomass with the $75^{\text {th }}$ percentile median and the $25^{\text {th }}$ percentile. Five replicates were performed for each phytoalexin. One-way ANOVA followed by Tukey's HSD test $(\mathrm{p}<0.01)$ was used to test the significance of differences.

As shown before, Verticillium has no capability to degrade brassinin (Fig. 13). Brassinin inhibited fungal growth (Fig. 20) has not affected spore germination (Fig. 19). Cyclobrassinin was has not affected fungal germination or growth (Fig. 19 and Fig. 20), probably because it was not stable in media (Fig. 16 and Fig. 17).

\subsection{Discussion}

This study describes the induction of phytoalexins in oilseed rape plants by $V$. longisporum infection, the toxicity of brassinin, brassilexin and cyclobrassinin to V. longisporum, and the ability of Verticillium species to detoxify these phytoalexins. 


\subsubsection{Elicitation of phytoalexins in B. napus by $V$. longisporum infection}

So far, fifty-four specialized metabolites (secondary metabolites) have been reported as phytoalexins from numerous species of Brassicaceae after different types of induction (Pedras and Abdoli 2017). B. napus alone produces a variety of phytoalexins when exposed to either abiotic (e.g. heavy metals) or biotic stress (e.g. fungal infection) (Pedras et al. 2011b). The function of phytoalexins is supposed to be the enhancement of plant resistance against pathogens (Kuć and Rush 1985). Therefore, it was of interest to determine whether $V$. longisporum infection induces phytoalexins in oilseed rape plants and if yes, which phytoalexins are induced. $V$. longisporum is a vascular pathogen that remains in xylem tissue until the host plant becomes senescent (Eynck et al. 2007), therefore detection of phytoalexins was attempted in whole plants and in xylem sap.

Table 4 summarizes elicitation information about phytoalexins that have been detected in oilseed rape plants according to literature and our study (Rouxel et al. 1991; Storck and Sacristán 1995; Dahiya and Rimmer 1988). The elicitation of brassinin and rutalexin in oilseed rape was not reported before. Our study shows that oilseed rape does not accumulate brassinin after $V$. longisporum infection, but is capable of synthesizing rutalexin. Rutalexin had been misassigned as cyclobrassinone in older publications, which was corrected by Pedras et al. (1999). 1-methoxybrassinin was under detectable level in this study. Other four previously reported phytoalexins of oilseed rape are brassilexin, cyclobrassinin, spirobrassinin and cyclobrassinin sulfoxide (Table 4). All of them, except cyclobrassinin sulfoxide, which we could not be monitored because of the lack of a standard, were elicited by $V$. longisporum infection and $\mathrm{CuSO}_{4}$ treatment in our study.

In addition to oilseed rape, over 30 other species of Brassicaceae have been reported to produce phytoalexins. Table 5 summarizes the published data on seven phytoalexins that were detected in this work. Phytoalexins elicited by $V$. longisporum in B. napus were also described in other Brassicaceae species. Brassilexin, cyclobrassinin, spirobrassinin, cyclobrassinin sulfoxide and 1methoxybrassinin were found in many crucifers, while brassinin and rutalexin are specific for Brassica spp.rape. Rutalexin has only been reported in B. napus ssp. rapifera; our study was the to show that it is also produced by B. napus. Rutalexin, spirobrassinin, cyclobrassinin and brassilexin were not detected in B. napus plants infected with $V$. longisporum in a previous study (Possienke 2012). 
Our results showed that the virulent strain VL43 of $V$. longisporum as well as avirulent strains VL1191 and VL1199 were able to elicit the synthesis of brassilexin, cyclobrassinin, spirobrassinin and rutalexin. 1-methoxybrassinin, which was reported to be produced by B. napus in response to fungal infection or abiotic stress (Pedras et al. 2007), was not detected in this study. Elicitation of phytoalexins by pathogens has been studied in many cruciferous plants, but surprisingly in B. napus only the effect of L. maculans has been published. The fact that apathogenic strains of $V$. longisporum induced phytoalexin synthesis in the same way as an aggressive strain was unexpected. The induction of phytoalexins by apathogenic fungal strains has not been studied so far. In line with the hypothesis about the function of phytoalexins (Hammeschmidt 1999), we expected that endophytes escaping detection by the host would not induce defense responses, which include phytoalexin synthesis. For a comparison, published data about the elicitation of these and further phytoalexins in cruciferous plants are summarized in Table 5. Oilseed rape cultivars may differ in the elicitation of phytoalexins, too. A rapid-cycling accession and variety Falcon were used in this study, while germline 2040 was used in the study on elicitation of phytoalexins in B. napus by L. maculans (Dahiya and Rimmer 1988).

Table 4. Induction of phytoalexin synthesis in B. napus by different elicitors

\begin{tabular}{lllll}
\hline & V. longisporum & CuSO & L. maculans & CuCl2 \\
Brassinin & No & No & - & - \\
Brassilexin & Yes & Yes & Yes & Yes \\
$\begin{array}{l}\text { Cyclobrassinin } \\
\text { Spirobrassinin }\end{array}$ & Yes & Yes & Yes & Yes \\
$\begin{array}{l}\text { Rutalexin } \\
\text { Cyclobrassinin }\end{array}$ & Yes & Yes & Yes & Yes \\
$\begin{array}{l}\text { sulfoxide } \\
\text { 1-methoxybrassinin }\end{array}$ & No & Yes & Yes & Yes \\
Source & This work & No & Yes & Yes \\
\hline
\end{tabular}

Yes: Oilseed rape plants synthesized the corresponding phytoalexin after elicitation

No: Oilseed rape plants failed to synthesize the corresponding phytoalexin to a detectable level after elicitation

-: no information available 
Table 5. Elicitation of seven main phytoalexins in Brassicaceae plants by various elicitors

\begin{tabular}{|c|c|c|c|c|c|c|c|}
\hline & Brassinin & Brassilexin & Cyclobrassinin & Spirobrassinin & $\begin{array}{l}\text { Cyclobrassinin } \\
\text { sulfoxide }\end{array}$ & Rutalexin & 1-methoxybrassinin \\
\hline B. adpressa & & & & & & & $\mathrm{Cu}, L m$ \\
\hline B. atlantica & & & & & & & $\mathrm{Cu}, L m$ \\
\hline $\begin{array}{l}\text { B. carinata } \\
\text { (Abyssinian } \\
\text { cabbage) }\end{array}$ & & $\mathrm{Cu}, L m$ & $\mathrm{Cu}, \mathrm{Lm}$ & $\mathrm{Cu}, L m$ & $\mathrm{Cu}, L m$ & & $\mathrm{Cu}, L m$ \\
\hline $\begin{array}{l}\text { B. juncea } \\
\text { (brown } \\
\text { mustard) }\end{array}$ & & $\begin{array}{l}\mathrm{Cu}, \mathrm{Ag}, \\
\mathrm{Ab}, \mathrm{Lm}\end{array}$ & $\mathrm{Cu}, L m$ & $\begin{array}{l}\mathrm{Cu}, \\
A b, L m\end{array}$ & $\mathrm{Cu}, L m$ & & \\
\hline B. montana & & & & & & & $\mathrm{Cu}, L m$ \\
\hline $\begin{array}{l}\text { B. napus } \\
\text { (oilseed rape) }\end{array}$ & & $\begin{array}{l}\mathrm{Cu}, L m \\
V l \\
\text { (this work) }\end{array}$ & $\begin{array}{l}\mathrm{Cu}, L m \\
V l \\
\text { (this work) }\end{array}$ & $\begin{array}{l}\mathrm{Cu}, L m \\
V l \\
\text { (this work) }\end{array}$ & $\mathrm{Cu}, L m$ & $\begin{array}{l}V l \\
\text { (this work) }\end{array}$ & $\mathrm{Cu}, L m$ \\
\hline $\begin{array}{l}\text { B. napis ssp. } \\
\text { rapifera } \\
\text { (rutabaga) }\end{array}$ & $\begin{array}{l}\mathrm{UV} \\
R s\end{array}$ & & & $\begin{array}{l}\mathrm{UV} \\
\text { Rs }\end{array}$ & & $\begin{array}{l}\mathrm{UV}, \\
R s\end{array}$ & $\begin{array}{l}\mathrm{UV} \\
R z\end{array}$ \\
\hline $\begin{array}{l}\text { B. nigra } \\
\text { (black } \\
\text { mustard) }\end{array}$ & & $\mathrm{Cu}, \mathrm{Lm}$ & $\mathrm{Cu}, L m$ & & $\mathrm{Cu}, L m$ & & \\
\hline $\begin{array}{l}\text { B. oleracea } \\
\text { var. } \\
\text { gongylodes } \\
\text { (kohlrabi) }\end{array}$ & & $\mathrm{Cu}, \mathrm{Lm}$ & & UV & & & UV \\
\hline $\begin{array}{l}\text { B. oleracea } \\
\text { var. capitata } \\
\text { (white } \\
\text { cabbage) }\end{array}$ & $P c$ & & $\begin{array}{l}\mathrm{Cu}, \\
P c\end{array}$ & $P c$ & & & $P c$ \\
\hline $\begin{array}{l}\text { B. oleracea } \\
\text { var. Botrytis } \\
\text { (cauliflower) }\end{array}$ & & & & UV & & & UV \\
\hline $\begin{array}{l}\text { B. rapa } \\
\text { (Chinese } \\
\text { cabbage) }\end{array}$ & $\begin{array}{l}\mathrm{UV}, \\
E c, P c\end{array}$ & & $\begin{array}{l}\mathrm{UV}, \\
E c, P c\end{array}$ & $\begin{array}{l}\mathrm{UV}, \\
E c, P c\end{array}$ & & & $P c$ \\
\hline $\begin{array}{l}\text { B. rapa } \\
\text { (rapseed) }\end{array}$ & & & $\mathrm{C} \mathrm{mu}, L m$ & & $\mathrm{Cu}$ & & $\mathrm{Cu}$ \\
\hline $\begin{array}{l}\text { B. rapa } \\
\text { (turnip) }\end{array}$ & & $\mathrm{Cu}, L m$ & $\begin{array}{l}\mathrm{UV} \\
\mathrm{Rs}\end{array}$ & UV & & & $\begin{array}{l}\mathrm{UV}, \\
R s\end{array}$ \\
\hline $\begin{array}{l}\text { R. sativus } \\
\text { (Japanese } \\
\text { radish) }\end{array}$ & $P c$ & & & $P c$ & & & $P c$ \\
\hline $\begin{array}{l}\text { S. arvensis } \\
\text { (white } \\
\text { mustard) }\end{array}$ & & $\mathrm{Lm}$ & & & $\mathrm{Lm}$ & & \\
\hline
\end{tabular}

The identity of brassinin, brassilexin, cyclobrassinin, spirobrassinin and rutalexin was confirmed by authentic standards from a commercial source or synthesized in the laboratory. The levels of most phytoalexins induced by $\mathrm{CuSO}_{4}$ were higher than the levels induced by $V$. longisporum infection. We assume that the larger fraction of the plant tissue affected by the $\mathrm{CuSO}_{4}$ treatment as comparison to V. longisporum infection accounted for the difference. Cyclobrassinin was a notable exception. We assume that in contrast to the other phytoalexins, the synthesis of cyclobrassinin was not restricted to the tissue which was directly affected by the pathogen, but that cyclobrassinin was also synthesized in distant tissues. This implies the existence of yet 
unknown signaling compound(s) that spread in the plant, eliciting defense responses in distant organs in a similar same way as in systemic acquired resistance (Ryals et al. 1996) or longdistance signaling in plants after wounding (Toyota et al. 2018).

\subsubsection{Degradation of phytoalexins by Verticillium spp.}

Pathogens adapted to their hosts are supposed to have developed the capability to degrade phytoalexins into non-toxic compounds. This study focused on the degradation of three cruciferous phytoalexins: brassinin, brassilexin, and cyclobrassinin. Brassilexin is one of the most toxic phytoalexins, and together with cyclobrassinin its synthesis was induced by $V$. longisporum infection. Brassinin is the most studied cruciferous phytoalexin with abundant information available. Therefore we involved brassinin in the degradation study, although it was not elicited by $V$. longisporum infection. The tested Verticillium species degraded brassilexin completely in less than $20 \mathrm{~h}$, while brassinin was still present in Verticillium cultures after $72 \mathrm{~h}$. This was surprising because most cruciferous pathogens studied so far were able to detoxify brassinin (Table 6). A possible explanation is that the ability to degrade brassinin was not selected during the adaptation of $V$. longisporum to crucifers. Brassinin synthesis in B. napus is not induced by V. longisporum infection. Cyclobrassinin degraded spontaneously in sterile media; interestingly, the degradation was slowed down in the presence of fungal cultures. This unusual "phytoalexin protection" has not been further investigated. We suggest that acidification of the medium by the fungus may account for this phenomenon. We cannot exclude that cyclobrassinin was enzymatically degraded by $V$. longisporum in spite of lower net degradation rate in fungal cultures as compared to sterile medium. The degradation of cyclobrassinin by $V$. longisporum cannot be addressed quantitatively until the spontaneous break-up and of the compound and its stabilization by fungal culture is explained.

Enzymes involved in brassinin detoxification have been studied in several plant pathogens. Brassinin oxidase (BOLm) was the first enzyme identified in L. maculans (isolate virulent on oilseed rape), which was capable to detoxifying phytoalexins in the tissue of oilseed rape (Pedras et al. 2008). Brassinin hydrolase (BHAb) from A. brassicicola and brassinin hydrolase (BHLm) from L. maculans (isolate virulent on mustard) were also reported (Pedras et al. 2009b). As mentioned previously, S. sclerotiorum detoxifies phytoalexins by glycosylation, and brassinin 
glucosyl transferase was identified in this fungus (Sexton et al. 2009). We have not detected glycosylation by $V$. longisporum or any other fungal species except $S$. sclerotiorum, which supports a view that in glycosylation is a rare detoxification mechanism in fungi. In addition to brassinin, cyclobrassinin hydrolase $(\mathrm{CHAb})$ was also isolated from A. brassicicola, but its characteristics were not identical to brassinin hydrolase (BHAb) (Pedras and Minic 2014), suggesting that pathogens possess detoxification enzymes that are specific for different phytoalexins.

To complicate matters further, some phytoalexins inhibit detoxifying enzymes. For example, cyclobrassinin, camalexin and wasalexins $\mathrm{A}$ and $\mathrm{B}$ inhibit brassinin oxidase (BOLm) (Pedras et al. 2008); cyclobrassinin inhibits brassinin hydrolases BHAb and BHLm (Pedras et al. 2009b); camalexin, dioxibrassinin and brassilexin inhibit cyclobrassinin hydrolase (CHAb) (Pedras and Minic 2014).

\subsubsection{Toxicity of phytoalexins to $V$. longisporum}

Among the phytoalexin tested, only brassilexin exhibited strong toxicity towards $V$. longisporum, completely inhibiting spore germination at $20 \mu \mathrm{g} / \mathrm{ml}$ (around $0.1 \mathrm{mM}$ ) (Fig.19). Brassilexin is known as one of the most toxic phytoalexins from crucifers, therefore the capability to efficiently degrade brassilexin may play an important role of fungal pathogenicity. Brassilexin was degraded by Verticillium mycelia fast (Fig. 9), which explains why mycelial growth was not affected by brassilexin up to $40 \mu \mathrm{g} / \mathrm{ml}$, as shown in Figure 20. Spores of $V$. longisporum apparently do not

possess the enzyme detoxifying brassinin, therefore. Because $V$. longisporum spores germinate outside the roots (Eynck et al. 2007), the production of brassilexin, even if it were constitutive or induced by previous infection with another pathogen, is unlikely to prevent infection of B. napus with V. longisporum.

Our study revealed that $V$. longisporum and other Verticillium species degrade brassilexin via an intermediate 3-(amino)methylenindoline-2-thione, which has been known from other degradation pathways (Pedras and Abdoli 2017) (Fig. 12). Further products could not be identified, presumably because the conversion of brassilexin to 3-(amino)methylenindoline-2-thione was a rate-limiting step in the degradation pathway and final products were too small to be detected by HPLC-MS. Our data indicates that Verticillium species may also produce brassicanal A as a 
product of a minor pathway of the degradation of brassilexin. To address the biological relevance of these two and possibly further degradation pathways, the enzymes and genes have to be identified to facilitate the construction of disruption mutants.

Although the role of brassilexin degradation in the virulence of Verticillium spp. can only be answered conclusively with the help of disruption mutants, our experiments indicate that detoxification of brassilexin plays a limited or no role in plant colonization because the ability to detoxify brassilexin was not correlated with the virulence of fungal strains. Figures 7 and 8 show that both virulent and avirulent Verticillium isolates degraded brassilexin with similar efficiency. Brassilexin degradation by the $V$. dahliae was the most efficient despite the fact that $V$. dahliae is not pathogenic on oilseed rape. Relatively high brassilexin degradation rates were also detected in F. graminearum and A. alternate (Fig. 6). Because brassilexin does not occur in plant hosts infected with these pathogens, we speculate that enzymes responsible for other processes are involved.

\section{References}

Aroud, H. (2013): Chemical interactions between Verticillium longisporum and oilseed rape Brassica napus. PhD dissertation, University of Goettingen, Goettingen, Germany. Available online at https://ediss.unigoettingen.de/handle/11858/00-1735-0000-0022-5EBD-7.

Budovská, M., Kudličková, Z., Kutschy, P., Pilátová, M., and Mojžiš, J. 2015. A facile method for the synthesis of indole phytoalexin rutalexin. Tetrahedron Letters. 56:3945-3947.

Budovská M, Pilátová MB, Tischlerová V, Mojžiš J. 2016. Spirocyclization reactions and antiproliferative activity of indole phytoalexins 1-methoxybrassinin and its 1-substituted derivatives. ARKIVOC 6, 198 - 234.

Cai, Xiang; Huang, Yongju; Jiang, Daohong; Fitt, Bruce D.L.; Li, Guoqing; Yang, Long (2018): Evaluation of oilseed rape seed yield losses caused by Leptosphaeria biglobosa in central China. In Eur J Plant Pathol 150 (1), pp. 179-190. DOI: 10.1007/s10658-017-1266-x.

Clarkson, John P.; Fawcett, Laura; Anthony, Steven G.; Young, Caroline (2014): A model for Sclerotinia sclerotiorum infection and disease development in lettuce, based on the effects of temperature, relative humidity and ascospore density. In PloS one 9 (4), e94049. DOI: 10.1371/journal.pone.0094049.

Csomós, P., Fodor, L., Sohár, P., and Bernáth, G. 2005. Synthesis of thiazino[6,5-b]indole derivatives, analogues of the phytoalexin cyclobrassinin. A new method for preparation of 3-aminomethylindole. Tetrahedron. 61:9257-9262.

Dahiya, Jagroop S.; Rimmer, S.Roger (1988): Phytoalexin accumulation in tissues of Brassica napus inoculated with Leptosphaeria maculans. In Phytochemistry 27 (10), pp. 3105-3107. DOI: 10.1016/0031-9422(88)80009-8.

Dunker, S.; Keunecke, H.; Steinbach, P.; Tiedemann, A. von (2008): Impact of Verticillium longisporum on Yield and Morphology of Winter Oilseed Rape (Brassica napus ) in Relation to Systemic Spread in the Plant. In $J$ Phytopathol 156 (11-12), pp. 698-707. DOI: 10.1111/j.1439-0434.2008.01429.x.

Eynck, C.; Koopmann, B.; Grunewaldt-Stoecker, G.; Karlovsky, P.; Tiedemann, A. von (2007): Differential interactions of Verticillium longisporum and V. dahliae with Brassica napus detected with molecular and histological techniques. In Eur J Plant Pathol 118 (3), pp. 259-274. DOI: 10.1007/s10658-007-9144-6. 
Fradin, Emilie F.; Thomma, Bart P. H. J. (2006): Physiology and molecular aspects of Verticillium wilt diseases caused by $V$. dahliae and V. albo-atrum. In Molecular plant pathology 7 (2), pp. 71-86. DOI: 10.1111/j.13643703.2006.00323.x.

Gross, D., Porzel, A., and Schmidt, J. 1994. Indole Phytoalexins from the Kohlrabi (Brassica oleracea var. gongylodes). Zeitschrift fuer Naturforschung. Section C. Biosciences. 49:281-285.

Hammerschmidt, R. 1999. Phytoalexins: what have we learned after 60 years? Annu Rev Phytopathol. 37:285-306.

Harper, F. R.; Berkenkamp, B. (1975): Rrevised growth-stage key for Brassica campestris and B. napus. In Can. J. Plant Sci. 55 (2), pp. 657-658. DOI: 10.4141/cjps75-103.

J.W. Bennett, L.L. Lasure (Eds.) (1991): More Gene Manipulations in Fungi, Academic Press, Inc, San Diego. pp. 444-445.

Janick, Jules (Ed.) (2002): Plant breeding reviews. Volume 22. Wiley InterScience (Online service). Hoboken, NJ: John Wiley \& Sons Inc (Plant breeding reviews, v. 22). Available online at http://dx.doi.org/10.1002/9780470650202.

Koh, Joshua C. O.; Barbulescu, Denise M.; Norton, Sally; Redden, Bob; Salisbury, Phil A.; Kaur, Sukhjiwan et al. (2017): A multiplex PCR for rapid identification of Brassica species in the triangle of U. In Plant methods 13, p. 49. DOI: $10.1186 / \mathrm{s} 13007-017-0200-8$.

Kuć, Joseph; Rush, Jeffrey S. (1985): Phytoalexins. In Archives of Biochemistry and Biophysics 236 (2), pp. 455472. DOI: 10.1016/0003-9861(85)90648-4.

Novakazi, Fluturë; Inderbitzin, Patrik; Sandoya, German; Hayes, Ryan J.; Tiedemann, Andreas Von; Subbarao, Krishna V. (2015): The Three Lineages of the Diploid Hybrid Verticillium longisporum Differ in Virulence and Pathogenicity. In Phytopathology 105 (5), pp. 662-673. DOI: 10.1094/PHYTO-10-14-0265-R.

Pedras, M. S. C.; Minic, Zoran; Jha, Mukund (2008): Brassinin oxidase, a fungal detoxifying enzyme to overcome a plant defense -- purification, characterization and inhibition. In The FEBS journal 275 (14), pp. 3691-3705. DOI: 10.1111/j.1742-4658.2008.06513.x.

Pedras, M. S. C., Montaut, S., and Suchy, M. 2004. Phytoalexins from the crucifer rutabaga: structures, syntheses, biosyntheses, and antifungal activity. J. Org. Chem. 69:4471-4476.

Pedras, M. Soledade C.; Abdoli, Abbas (2017): Pathogen inactivation of cruciferous phytoalexins. Detoxification reactions, enzymes and inhibitors. In $R S C A d v .7$ (38), pp. 23633-23646. DOI: 10.1039/C7RA01574G.

Pedras, M. Soledade C.; Chumala, Paulos B.; Jin, Wei; Islam, Mohammed S.; Hauck, Dominic W. (2009a): The phytopathogenic fungus Alternaria brassicicola. Phytotoxin production and phytoalexin elicitation. In Phytochemistry 70 (3), pp. 394-402. DOI: 10.1016/j.phytochem.2009.01.005.

Pedras, M. Soledade C.; Hossain, Mohammad (2006): Metabolism of crucifer phytoalexins in Sclerotinia sclerotiorum. Detoxification of strongly antifungal compounds involves glucosylation. In Organic \& biomolecular chemistry 4 (13), pp. 2581-2590. DOI: 10.1039/b604400j.

Pedras, M. Soledade C.; Hossain, Sajjad; Snitynsky, Ryan B. (2011a): Detoxification of cruciferous phytoalexins in Botrytis cinerea. Spontaneous dimerization of a camalexin metabolite. In Phytochemistry 72 (2-3), pp. 199-206. DOI: 10.1016/j.phytochem.2010.11.018.

Pedras, M. Soledade C.; Minic, Zoran (2014): The phytoalexins brassilexin and camalexin inhibit cyclobrassinin hydrolase, a unique enzyme from the fungal pathogen Alternaria brassicicola. In Bioorganic \& medicinal chemistry 22 (1), pp. 459-467. DOI: 10.1016/j.bmc.2013.11.005.

Pedras, M. Soledade C.; Minic, Zoran; Sarma-Mamillapalle, Vijay K. (2009b): Substrate specificity and inhibition of brassinin hydrolases, detoxifying enzymes from the plant pathogens Leptosphaeria maculans and Alternaria brassicicola. In The FEBS journal 276 (24), pp. 7412-7428. DOI: 10.1111/j.1742-4658.2009.07457.x.

Pedras, M. Soledade C.; Sorensen, John L.; Okanga, Francis I.; Zaharia, Irina L. (1999): Wasalexins A and B, new phytoalexins from wasabi. Isolation, synthesis, and antifungal activity. In Bioorganic \& medicinal chemistry letters 9 (20), pp. 3015-3020. DOI: 10.1016/S0960-894X(99)00523-5. 
Pedras, M. Soledade C.; Suchy, Mojmir (2005): Detoxification pathways of the phytoalexins brassilexin and sinalexin in Leptosphaeria maculans. Isolation and synthesis of the elusive intermediate 3-formylindolyl-2-sulfonic acid. In Organic \& biomolecular chemistry 3 (10), pp. 2002-2007. DOI: 10.1039/b501907a.

Pedras, M. Soledade C.; Yaya, Estifanos E.; Glawischnig, Erich (2011b): The phytoalexins from cultivated and wild crucifers. Chemistry and biology. In Natural product reports 28 (8), pp. 1381-1405. DOI: 10.1039/c1np00020a.

Pedras, M. Soledade C.; Zheng, Qing-an; Sarma-Mamillapalle, Vijay K. (2019): The Phytoalexins from Brassicaceae. Structure, Biological Activity, Synthesis and Biosynthesis. In Natural Product Communications 2 (3), 1934578X0700200. DOI: 10.1177/1934578X0700200315.

Possienke, M. (2012): Signals and metabolic consequences during the interaction of Brassicaceae and Verticillium longisporum. PhD dissertation, University of Goettingen, Goettingen, Germany. Available online at http://hdl.handle.net/11858/00-1735-0000-000D-F0E8-3.

Ratzinger, Astrid (2008): Development and application of LC-MS-based differential metabolic profiling in plant systems. PhD dissertation. Georg August University, Göttingen, Germany. Available online at http://hdl.handle.net/11858/00-1735-0000-0006-B024-7.

Ratzinger, Astrid; Riediger, Nadine; Tiedemann, Andreas Von; Karlovsky, Petr (2009): Salicylic acid and salicylic acid glucoside in xylem sap of Brassica napus infected with Verticillium longisporum. In Journal of plant research 122 (5), pp. 571-579. DOI: 10.1007/s10265-009-0237-5.

Rouxel, T.; Kollmann, A.; Boulidard, L.; Mithen, R. (1991): Abiotic elicitation of indole phytoalexins and resistance to Leptosphaeria maculans within Brassiceae. In Planta 184 (2), pp. 271-278. DOI: 10.1007/BF00197957.

Ryals, J. A.; Neuenschwander, U. H.; Willits, M. G.; Molina, A.; Steiner, H. Y.; Hunt, M. D. (1996): Systemic acquired resistance. In The Plant Cell, 8(10), pp. 1809-1819.Scholander, P. F.; Bradstreet, E. D.; Hemmingsen, E. A.; Hammel, H. T. (1965): Sap Pressure in Vascular Plants. Negative hydrostatic pressure can be measured in plants. In Science (New York, N.Y.) 148 (3668), pp. 339-346. DOI: 10.1126/science.148.3668.339.

Sexton, Adrienne C.; Minic, Zoran; Cozijnsen, Anton J.; Pedras, M. Soledade C.; Howlett, Barbara J. (2009): Cloning, purification and characterisation of brassinin glucosyltransferase, a phytoalexin-detoxifying enzyme from the plant pathogen Sclerotinia sclerotiorum. In Fungal genetics and biology : FG \& B 46 (2), pp. 201-209. DOI: 10.1016/j.fgb.2008.10.014.

Soledade, M.; Pedras, C.; Taylor, Janet L. (1993): Metabolism of the Phytoalexin Brassinin by the "Blackleg" Fungus. In J. Nat. Prod. 56 (5), pp. 731-738. DOI: 10.1021/np50095a010.

Storck, Michael; Sacristán, Maria D. (1995): The Role of Phytoalexins in the Seedling Resistance to Leptosphaeria maculans in Some Crucifers. In Zeitschrift für Naturforschung C 50 (1-2), pp. 15-20. DOI: 10.1515/znc-1995-1-204.

Strehlow, Becke; Mol, Friederike de; Struck, Christine (2015): Risk Potential of Clubroot Disease on Winter Oilseed Rape. In Plant disease 99 (5), pp. 667-675. DOI: 10.1094/PDIS-05-14-0482-RE.

Takasugi, Mitsuo; Katsui, Nobukatsu; Shirata, Akira (1986): Isolation of three novel sulphur-containing phytoalexins from the chinese cabbage Brassica campestris L. ssp. pekinensis(cruciferae). In J. Chem. Soc., Chem. Commun. (14), p. 1077. DOI: 10.1039/c39860001077.

Toyota, M., Spencer, D., Sawai-Toyota, S., Jiaqi, W., Zhang, T., Koo, A. J., et al. (2018). Glutamate triggers longdistance, calcium-based plant defense signaling. In Science. 361, pp. 1112-1115.

VanEtten, H. D.; Mansfield, J. W.; Bailey, J. A.; Farmer, E. E. (1994): Two Classes of Plant Antibiotics. Phytoalexins versus "Phytoanticipins". In The Plant cell 6 (9), pp. 1191-1192. DOI: 10.1105/tpc.6.9.1191.

Zeise, K.; Tiedemann, A. von (2002a): Application of RAPD-PCR for Virulence Type Analysis within Verticillium dahliae and V. longisporum. In J Phytopathol 150 (10), pp. 557-563. DOI: 10.1046/j.1439-0434.2002.00799.x.

Zeise, K.; Tiedemann, A. von (2002b): Host Specialization among Vegetative Compatibility Groups of Verticillium dahliae in Relation to Verticillium longisporum. In J Phytopathol 150 (3), pp. 112-119. DOI: 10.1046/j.14390434.2002.00730.x. 


\section{Article III: Bis-naphthopyrone pigments protect filamentous ascomycetes from a wide range of predators}

Yang $\mathrm{Xu}^{1}$, Maria Vinas ${ }^{1,2}$, Albatol Alsarrag ${ }^{1}$, Ling Su${ }^{1}$, Katharina Pfohl ${ }^{1}$, Marko Rohlfs ${ }^{3}$,

Wilhelm Schäfer ${ }^{4}$, Wei Chen ${ }^{5 *}$, Petr Karlovsky ${ }^{1 *}$

The article was published online in Nature Communications 10 (1), p.3579 in August 2019. The supplementary materials are attached to the main part. The full article can be found online at:

https://www.nature.com/articles/s41467-019-11377-5

DOI: $\underline{10.1038 / \mathrm{s} 41467-019-11377-5}$

\section{Author contribution:}

Ling Su carried out the feeding experiments with nematodes and its related RNA extraction and RT-qPCR in order to determine the induction of Aurofusarin. Besides, she was also involved in the experiment design and data analysis. 
ARTICLE

https://doi.org/10.1038/s41467-019-11377-5

\title{
Bis-naphthopyrone pigments protect filamentous ascomycetes from a wide range of predators
}

\author{
Yang Xu1 ${ }^{1}$, Maria Vinas (1) ${ }^{1,2}$, Albatol Alsarrag ${ }^{1}$, Ling Su${ }^{1}$, Katharina Pfohl ${ }^{1}$, Marko Rohlfs ${ }^{3}$, Wilhelm Schäfer ${ }^{4}$, \\ Wei Chen $^{5} \&$ Petr Karlovsky (1) ${ }^{1}$
}

It is thought that fungi protect themselves from predation by the production of compounds that are toxic to soil-dwelling animals. Here, we show that a nontoxic pigment, the bisnaphthopyrone aurofusarin, protects Fusarium fungi from a wide range of animal predators. We find that springtails (primitive hexapods), woodlice (crustaceans), and mealworms (insects) prefer feeding on fungi with disrupted aurofusarin synthesis, and mealworms and springtails are repelled by wheat flour amended with the fungal bis-naphthopyrones aurofusarin, viomellein, or xanthomegnin. Predation stimulates aurofusarin synthesis in several Fusarium species and viomellein synthesis in Aspergillus ochraceus. Aurofusarin displays low toxicity in mealworms, springtails, isopods, Drosophila, and insect cells, contradicting the common view that fungal defence metabolites are toxic. Our results indicate that bisnaphthopyrones are defence compounds that protect filamentous ascomycetes from predators through a mechanism that does not involve toxicity.

\footnotetext{
${ }^{1}$ University of Goettingen, Molecular Phytopathology and Mycotoxin Research, 37077 Göttingen, Germany. ${ }^{2}$ CIGRAS, University of Costa Rica, 2060 San Pedro, Costa Rica. ${ }^{3}$ University of Bremen, Institute of Ecology, Population and Evolutionary Ecology Group, 28359 Bremen, Germany. ${ }^{4}$ University of Hamburg, Biocenter Klein Flottbek, Molecular Phytopathology and Genetics, 22609 Hamburg, Germany. ${ }^{5}$ Zhejiang University, College of Biosystems Engineering and Food Science, Department of Food Science and Nutrition, Hangzhou 310058, P.R. China. Correspondence and requests for materials should be addressed to W.C. (email: zjuchenwei@zju.edu.cn) or to P.K. (email: pkarlov@gwdg.de)
} 
S oil fungi play a key role in nutrient cycling by degrading recalcitrant plant biomass. Fungal biomass is an attractive source of nutrients for soil invertebrates ${ }^{1}$, and predation on fungi disrupts fungal networks ${ }^{2,3}$ and modulates the composition $^{4}$ and activity ${ }^{5}$ of fungal communities, thereby affecting fungal ecosystem services ${ }^{6}$. Because fungi are sessile organisms, their protection from predation consists primarily of chemical defence.

This chemical defence can be mediated by proteins or secondary metabolites. The role of fungal ribosome-inactivating proteins $^{7}$, protease inhibitors ${ }^{8}$, and lectins $s^{9,10}$ in fungal chemical defence has been elucidated at the molecular level. Studies of fungal defence metabolites have a long history, albeit with inconclusive outcomes. In 1977, Daniel Janzen suggested that fungal toxins protect moulded material from consumption by large animals and hinted that the same metabolites may protect infected grain from storage pests ${ }^{11}$. Janzen's ideas led to the hypothesis that mycotoxins protect fungi from predators, and the insecticidal properties of many mycotoxins have since been studied $^{12-14}$. Apart from their toxicity to insects, circumstantial support for the role of mycotoxins in defence against predators has been drawn from the stimulation of mycotoxin synthesis by arthropod grazing ${ }^{15}$ and mechanical injury ${ }^{16}$ and from the accumulation of toxic metabolites in fungal reproductive

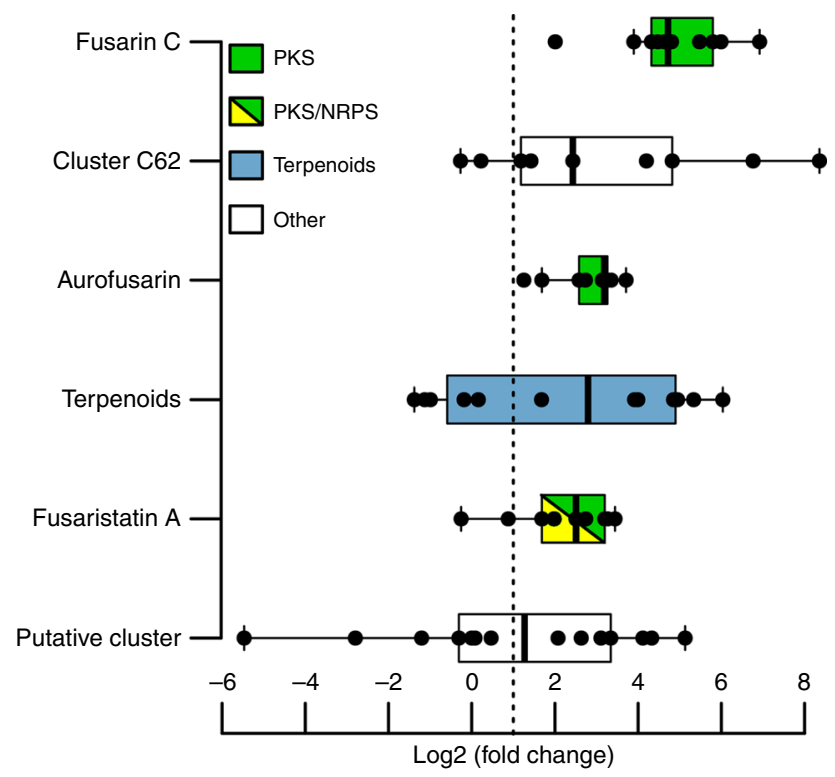

Fig. 1 Secondary metabolite pathways upregulated by grazing in $F$. graminearum. Fusarium graminearum IFA66 was exposed to the springtail Folsomia candida for $48 \mathrm{~h}$. RNA was extracted, and the levels of individual mRNAs were determined by RNA sequencing (RNAseq). Black points show $\log _{2}$ (fold change) values for each gene in grazed versus control cultures. Upregulated gene clusters are defined as having $>50 \%$ of all genes and/or having the gene that encodes a signature enzyme be significantly induced (the $\log _{2}$ (FPKM) was higher than 1.0 (dotted line), and the $q$ value was lower than 0.01). Accession numbers: fusarin C (FGSG_07798, 07800-07805, and 13222-13224), cluster C62 (FGSG_10606, 10608, 10609, and 10611-10614, 10616, and 10617), aurofusarin (FGSG_02320-02329), terpenoids (FGSG_01737-01749), fusaristatin A (FGSG_08204-08210, 08213, and 08214), and putative cluster (FGSG_10557-10560, 10562-10567, 10569-10571, and 10573). Four biological replicates were used. Box plots show the median and interquartile range. Whiskers indicate the largest and smallest observation or 1.5 -fold of the interquartile range, whichever is smaller or larger, respectively $(\mathrm{Q} 1-1.5 \times(\mathrm{Q} 3-\mathrm{Q} 1)$ or $\mathrm{Q} 3+1.5 \times(\mathrm{Q} 3-\mathrm{Q} 1))$. Source data are provided in a Source Data file organs ${ }^{17}$. Although the ecological function of toxins accumulating in mushrooms (fruiting bodies of basidiomycetes) has been elucidated $^{18}$, efforts to substantiate the function of major mycotoxins of filamentous ascomycetes in their defence against predators have remained inconclusive ${ }^{19,20}$. Mycotoxin gliotoxin facilitates the escape of Aspergillus flavus during phagocytosis by a soil amoeba $^{21}$; however, whether gliotoxin protects its producers from animal predators remains unknown. Two polyketides that have not been determined to be mycotoxins have been shown to protect two ascomycetes fungi from animal predation: Asparasone has protected the sclerotia of Aspergillus flavus from sap beetles $^{22}$, and neurosporin A has protected Neurospora crassa from springtail grazing 23 . Nevertheless, there is no indication that these findings can be generalised to related metabolites, other fungal species, or additional predators.

In this work, we investigate the effect of springtail grazing on the transcriptome of the filamentous ascomycete Fusarium graminearum (F. graminearum). The biosynthesis pathways for several secondary metabolites are induced via grazing. One of these metabolites is aurofusarin, which belongs to bisnaphthopyrones that are produced by many ascomycetes. Predation and mechanical damage stimulate aurofusarin synthesis. When mutants of $F$. graminearum with disrupted aurofusarin synthesis are offered to springtails, isopods, and mealworms, all predators strongly prefer the mutants over the aurofusarinproducing strains. Food choice experiments with purified aurofusarin, xanthomegnin, and viomellein-which are bisnaphthopyrones produced by many species of Fusarium, Aspergillus and Penicillium-reveal antifeedant effects of all three metabolites in mealworms and springtails. Toxicity assays with mealworms, springtails, Drosophila larvae, and insect cell cultures show a low toxicity of aurofusarin to arthropods. These results suggest that fungal bis-naphthopyrone pigments-which are widespread among ascomycetes-protect fungi from predators by exerting antifeedant effects on a wide range of phylogenetically distant arthropods.

\section{Results}

Predation stimulates the synthesis of aurofusarin. Assuming that defence metabolites are synthesised on demand, we sequenced the transcriptome of the fungus $F$. graminearum that had been exposed to the springtail Folsomia candida to reveal which biosynthetic pathways were induced by grazing. RNA was extracted from fungal cultures after grazing, and the mRNA levels of all genes were determined by sequencing (RNAseq). Grazing was found to stimulate the transcription of pathways for the metabolites aurofusarin, fusarin C, and fusaristatin A (Fig. 1 and Supplementary Fig. 1b, c), additional gene clusters that are putatively involved in secondary metabolism (Supplementary Fig. 2), and seven genes that encode small proteins (Supplementary Fig. 1a). Pathways for the mycotoxins deoxynivalenol and zearalenone-which are toxic to insects ${ }^{13,14}$ - and for necrosis and ethylene-inducing peptide-like proteins-which we hypothesised to be defence agents owing to their similarity to lectins ${ }^{24}$-were not induced by grazing. The RNAseq data are accessible under E-MTAB-6939 at ArrayExpress, EMBL-EBI (www.ebi.ac.uk/arrayexpress), and their analysis for 13,710 genes of F. graminearum can be found in Supplementary Data 1.

Aurofusarin was selected for further work because it is produced by many fungal species ${ }^{25,26}$ and because metabolites of similar structures are produced by many genera of ascomycetes $^{27}$ (see below). Aurofusarin is a red pigment known from maize ears infected with F. graminearum (Fig. 2a) and pure cultures of the fungus (Fig. 2b). It belongs to dimeric naphtho- $\gamma$ pyrones (Fig. 2f). Springtail grazing stimulated the transcription 
a

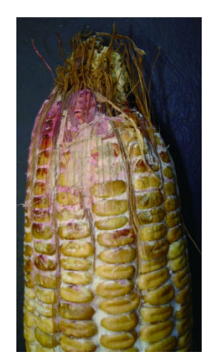

b

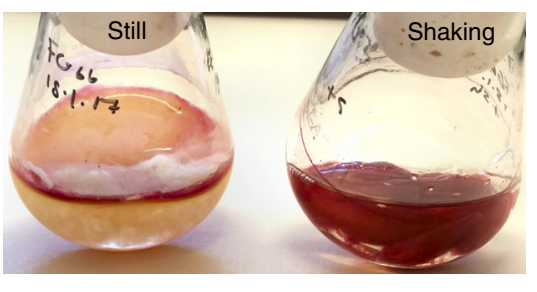

C

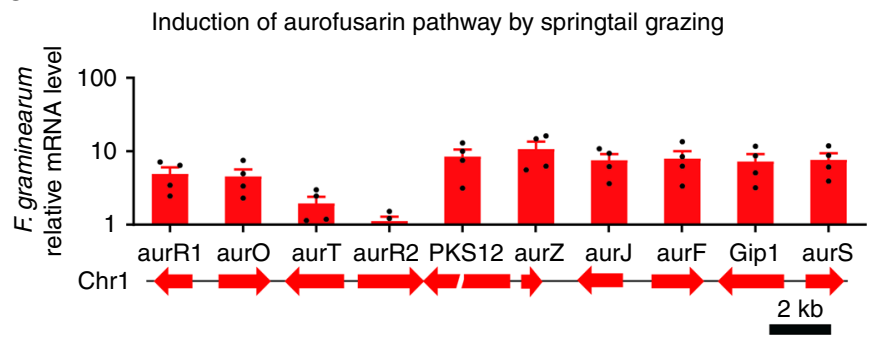

d

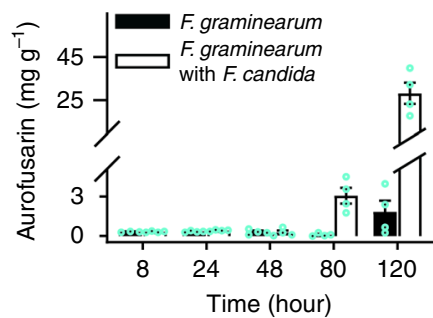

e

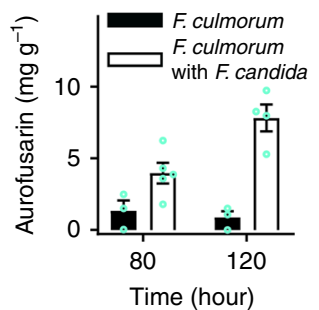

f<smiles></smiles>

g

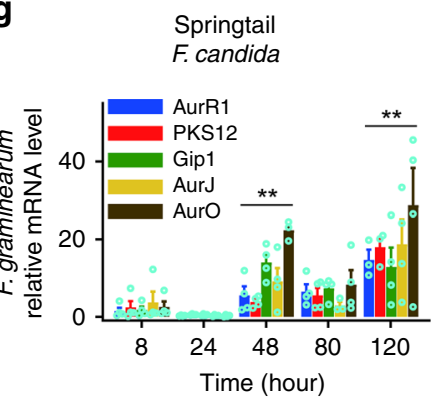

h

h Springtail

F. candida

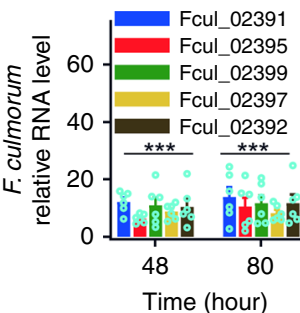

i

Nematode

A. saprophilus

\section{j}

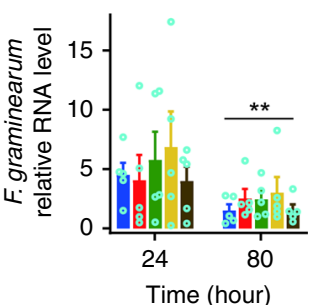

Nematode B. mucronatus

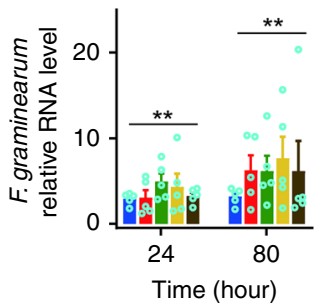

Fig. 2 Aurofusarin synthesis in Fusarium spp. is stimulated by predation. a An ear of corn inoculated with F. graminearum showing red pigment aurofusarin (courtesy of Dr Belinda J. van Rensburg, ARC South Africa). b F. graminearum cultures in potato broth. $\mathbf{c}$ Upregulation of genes of aurofusarin biosynthesis in F. graminearum after exposure to grazing by the springtail Folsomia candida for $48 \mathrm{~h}$ (RNAseq; $n=4$; see Fig. 1 for details). d, e Aurofusarin accumulation in F. graminearum and F. culmorum exposed to grazing by the springtail Folsomia candida $(n=4)$. $\mathbf{f}$ Structure of aurofusarin. $\mathbf{g}$, $\mathbf{h}$ Upregulation of genes of aurofusarin synthesis in F. graminearum and F. culmorum after grazing by Folsomia candida (RT qPCR). $\mathbf{i}$, $\mathbf{j}$ Upregulation of genes of aurofusarin synthesis after exposure to the fungivorous nematodes Aphelenchoides saprophilus and Bursaphelenchus mucronatus (RT qPCR). The gene cluster was labelled as significantly induced when mRNA levels of at least three genes increased at least threefold and the increase was statistically significant $\left({ }^{\star \star} P<0.001,{ }^{\star \star \star} P<\right.$ 0.0001 , two-tailed $t$ test) with both reference genes (glyceraldehyde-3-phosphate dehydrogenase and elongation factor 1a). Error bars show s.e.m. Three to four biological replicates were used in RT qPCR (see Supplementary Data 3). Source data are provided in a Source Data file

of all genes of the aurofusarin cluster except one (Fig. 2c). To examine whether aurofusarin synthesis was also induced by other predators, F. graminearum and F. culmorum were subjected to feeding by the springtail $F$. candida and the nematodes Aphelenchoides saprophilus and Bursaphelenchus mucronatus for different time periods, and relative mRNA levels for five genes of aurofusarin synthesis ${ }^{28}$ were estimated by RT qPCR (Fig. $2 \mathrm{~g}-\mathrm{j}$ ). Predation induced the aurofusarin pathway in all fungus/animal combinations. The estimation of the aurofusarin concentration in F. graminearum and F. culmorum cultures that had been subjected to springtail grazing by high-performance liquid chromatography (HPLC) with light absorption detection (HPLC-DAD) revealed that aurofusarin accumulation was simulated by grazing and that aurofusarin in grazed mycelia amounted to up to $2.5 \%$ of the dry weight (Fig. $2 \mathrm{~d}$, e). We were not aware of any non-polymeric secondary metabolite that accumulates in fungal mycelia at such a level, and we therefore determined the aurofusarin content in the mycelia of five Fusarium species grown in liquid cultures by HPLC with mass spectrometric detection (HPLC-MS/MS) (Supplementary Fig. 3). Aurofusarin levels of $1-7 \%$ of dry weight were found in four Fusarium species. Because both HPLC-DAD and HPLC-MS rely on aurofusarin standards, which are notoriously unstable (see Methods), extracts of six F. venenatum cultures were reanalysed via HPLC with evaporative light-scattering detection (ELSD) for additional verification. ELSD is less accurate than $\mathrm{DAD}$ or MS yet does not require aurofusarin standards. The analysis confirmed the high levels of aurofusarin in fungal mycelia.

To determine whether aurofusarin synthesis is stimulated by predation in other Fusarium species, cultures of $F$. poae, $F$. venenatum, and $F$. avenaceum on solid media were subjected to grazing by the springtail $F$. candida, and $F$. venenatum and $F$. sporotrichioides were subjected to grazing by the woodlouse Porcellio scaber (Supplementary Fig. 4). Mycelia of F. venenatum, F. sporotrichioides, and F. avenaceum turned red in areas exposed to predation, indicating that the predation had stimulated aurofusarin synthesis.

Aurofusarin deters a wide range of predators from feeding. A key characteristic of defence metabolites is that they suppress predation. To test whether aurofusarin protected its producers from predation, F. graminearum accumulating aurofusarin and genetically engineered strains that were unable to produce aurofusarin were simultaneously offered to predators in food choice experiments (Fig. 3). Predators representing distant arthropod 
a

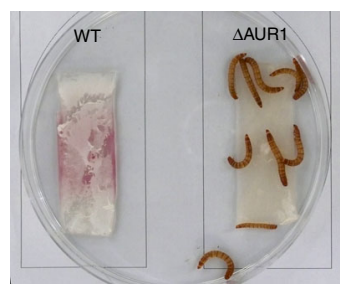

C

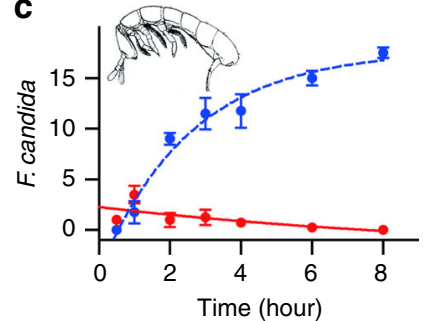

e
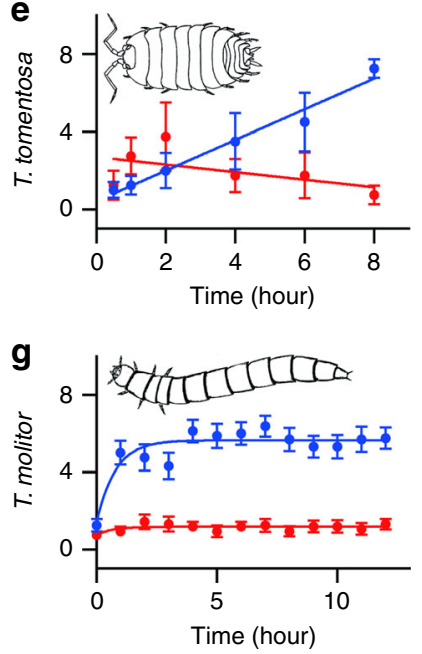

Fig. 3 Predators avoid fungal cultures in which aurofusarin accumulates. a Mealworms in a Petri dish with cultures of Fusarium graminearum producing aurofusarin (WT) and a nonproducing mutant ( $\triangle$ AUR1). b-d The food preferences of the springtail Folsomia candida for $F$. graminearum WT and aurofusarin-nonproducing mutants were studied by placing springtails that had been starved for 2 days into the centre of a Petri dish containing fungal cultures and by counting the animals feeding on each culture (20 animals per plate; four replicates). e-f The food preference of the isopod Trichorhina tomentosa was tested in the same manner with eight animals per arena and four replicates. $\mathbf{g}$ The food preference of the mealworm Tenebrio molitor was examined by placing larvae into Petri dishes containing fungal cultures on microscope slides, as shown in a. Sixteen replicates with 10 animals per plate were used. Error bars show $95 \% \mathrm{Cl}$. Source data are provided in a Source Data file

lineages were used: the collembolan F. candida (primitive arthropod), the woodlouse Trichorhina tomentosa (crustacean), and the mealworm Tenebrio molitor (insect). Aurofusarinproducing and nonproducing cultures were placed onto opposite sides of Petri dishes, and the number of animals feeding on each culture was monitored. All predators displayed a strong preference for mutants that did not produce aurofusarin (Fig. 3). Within 1 hour, most mealworms had gathered on the cultures without aurofusarin, where they remained until the end of the experiment. The springtails and woodlice gradually gathered on cultures of non-producers; as shown in Fig. 3, after $8 \mathrm{~h}$ most animals were feeding on cultures without aurofusarin. The disruption of biosynthetic pathways for the mycotoxins deoxynivalenol and zearalenone in F. graminearum had no effect on food preference (Supplementary Fig. 5), though both mycotoxins are toxic to insects ${ }^{13,14}$. The reversal of the springtails' food preference for $F$. verticillioides over $F$. graminearum via the disruption of aurofusarin synthesis in F. graminearum (Supplementary Fig. 5) indicates that aurofusarin had served as the major-or only-defence metabolite of F. graminearum deterring the springtails in this experiment.

The disruption of the biosynthetic pathway for aurofusarin synthesis may indirectly affect the synthesis of other metabolites 25,29 which may include unknown attractants. To clarify whether indirect effects of the disruption of the aurofusarin pathway may account for the arthropods' preference for fungi in which aurofusarin does not accumulate, mealworms were offered wheat flour amended with purified aurofusarin and unamended flour (Fig. 4). The larvae's strong preference for flour without aurofusarin revealed that aurofusarin possesses antifeedant activity and efficiently deters predators at a concentration similar to its concentration in fungal mycelia upon grazing (Fig. $2 \mathrm{~d}, \mathrm{e}$ ). The exclusion of light in these experiments helped ensure that the animals not recognise aurofusarin by its colour.

Aurofusarin is not toxic to arthropods. Why do predators avoid aurofusarin-accumulating fungi? The avoidance of food containing toxins is an adaptation that reduces toxic exposure ${ }^{7}$. Aurofusarin has been reported to be toxic in poultry ${ }^{30}$, but metabolites other than aurofusarin might have been responsible for the effects described in this work because the poultry feed used in these trials had not been amended with pure aurofusarin, but rather with a culture of a fungus known to be a potent producer of mycotoxins. To determine whether aurofusarin is toxic to insects, mealworms were fed wheat flour amended with aurofusarin for $10 \mathrm{~d}$, and their weight gain was determined. Aurofusarin at concentrations of up to $1 \mathrm{mg} \mathrm{g}^{-1}$ did not affect the mealworms' growth (Fig. 5a). This concentration is two to three orders of magnitude greater than concentrations at which mycotoxins display toxicity in insects ${ }^{13,14,31}$. At $10 \mathrm{mg} \mathrm{g}^{-1}$, aurofusarin suppressed the mealworms' growth. At this concentration, aurofusarin substantially reduced feed intake (Fig. 4a), and we therefore assume that the suppression of mealworms' growth on flour with $10 \mathrm{mg} \mathrm{g}^{-1}$ of aurofusarin was caused by reduced feeding rather than toxicity.

To test the developmental toxicity of aurofusarin on an arthropod that did not feed on filamentous fungi, we fed larvae of Drosophila melanogaster with food amended with aurofusarin at the same level as in the previous experiments as well as at a lower level of $2 \mathrm{mg} \mathrm{g}^{-1}$ for 2 days. This feeding was followed by a transfer to a medium without aurofusarin to accomplish the development (Fig. 5b). No differences in the number of adults emerging from pupae in feeding trials with and without aurofusarin were found, which indicated that aurofusarin did not cause developmental toxicity in D. melanogaster.

The effect of aurofusarin on the growth and mortality of the springtail F. candida and the woodlice Trichorhina tomentosa was studied by monitoring the mortality of animals fed on F. graminearum and its aurofusarin-nonproducing mutant for 5 weeks, and the size of the animal bodies was estimated at the end of the experiment (Table 1). Forced feeding on F. graminearum cultures in which aurofusarin had accumulated did not cause any mortality in the springtail F. candida or the isopod Trichorhina tomentosa. The growth of animals fed on mycelia with aurofusarin was reduced as compared with aurofusarin-nonproducing mutants, but the effects were small, indicating that reduced feed consumption rather than toxicity was the cause. The lack of mortality during 5 weeks of feeding on 

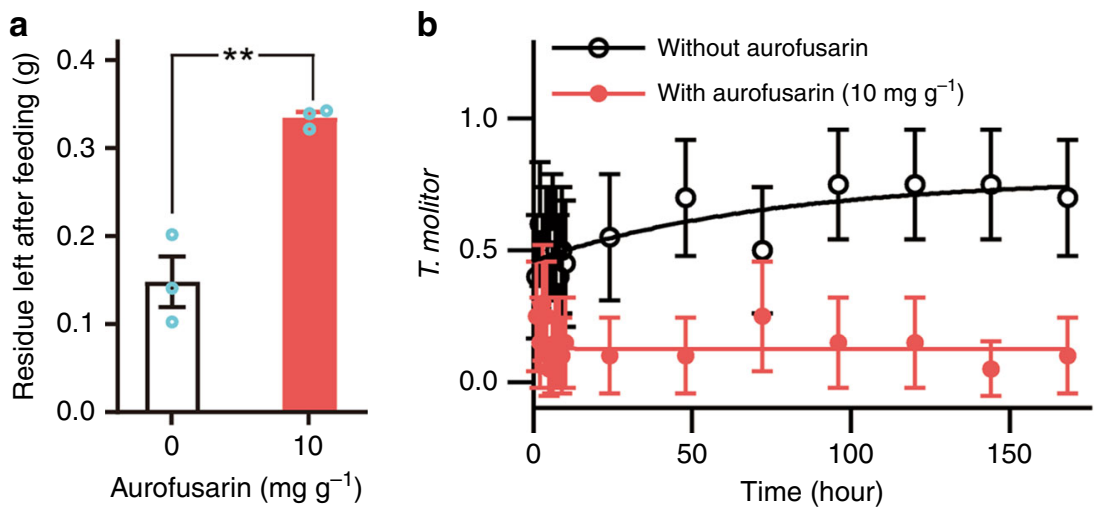

Fig. 4 Aurofusarin in wheat flour repels mealworms. a The weight of the wheat flour and faeces left by five larvae of $T$. molitor after $4 \mathrm{~d}$ of feeding on $0.4 \mathrm{~g}$ of wheat flour with and without $10 \mathrm{mg} \mathrm{g}^{-1}$ of aurofusarin. The significance of the difference was analysed with unpaired two-tailed $t$ test $(n=3, p=$ 0.0033). b Single mealworms were placed on Petri dishes between two portions of $100 \mathrm{mg}$ of wheat flour, one of which was amended with $10 \mathrm{mg} \mathrm{g}^{-1}$ of aurofusarin. The arenas were kept in total darkness and opened only for a second in dim light to record the mealworm location $(n=20$, error bars show $\mathrm{Cl}$ 95\%). Source data are provided in a Source Data file

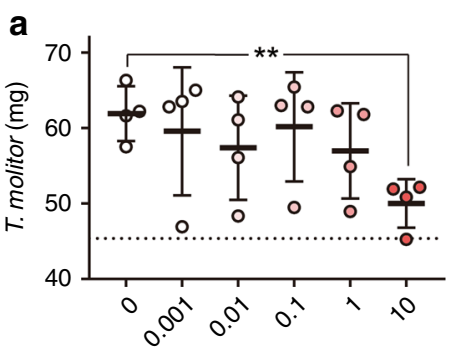

Aurofusarin $\left(\mathrm{mg} \mathrm{g}^{-1}\right)$

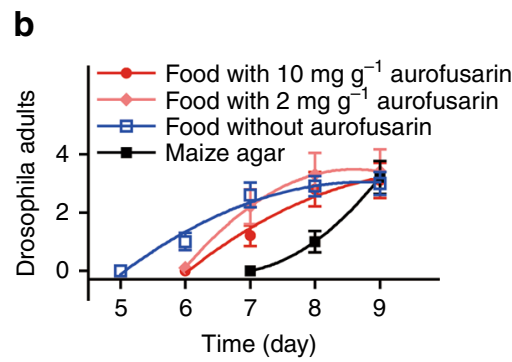

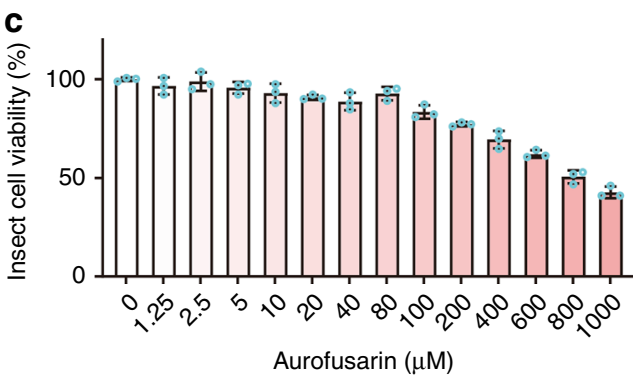

Aurofusarin $(\mu \mathrm{M})$

Fig. 5 Toxicity of aurofusarin to arthropods. a The weight of $T$. molitor after $10 \mathrm{~d}$ of feeding on wheat with different concentrations of aurofusarin. The average initial weight is indicated by the dashed line. Means and SD of 4 replicates, each with 10 animals, are shown. The effect of aurofusarin at 10 mg $g^{-1}$ was analysed with two-sided $t$ tests (10 individuals per experiment, $n=4, p=0.0027$ ). $\mathbf{b}$ The developmental toxicity of aurofusarin was tested by feeding larvae of Drosophila melanogaster on food with and without aurofusarin for two days, followed by incubation on standard food to accomplish the development (10 larvae per arena, 10 arenas per treatment, error bars show s.e.m.). Maize agar was used to simulate starvation. c The viability of Sf9 cells (fall armyworm Spodoptera frugiperda) after $24 \mathrm{~h}$ of incubation with aurofusarin $(n=3)$ shown as means with error bars showing s.e.m. The coloration of data points and bars indicates aurofusarin concentration in flour. Source data are provided in a Source Data file

Table 1 Growth of predators fed aurofusarin mutants of Fusarium graminearum

\begin{tabular}{|c|c|c|c|c|c|c|c|c|}
\hline \multirow{2}{*}{ F. candida } & $\triangle$ AUR1 & 21 & $1.08 \pm 0.20$ & $121 \%$ & $<0.0001$ & $0.23 \pm 0.06$ & $123 \%$ & $<0.0001$ \\
\hline & $\triangle A \cup R 2$ & 35 & $1.05 \pm 0.23$ & $119 \%$ & $<0.0001$ & $0.23 \pm 0.06$ & $122 \%$ & $<0.0001$ \\
\hline \multirow[t]{3}{*}{ T. tomentosa } & WT & 10 & $1.50 \pm 0.13$ & $100 \%$ & - & $0.62 \pm 0.07$ & $100 \%$ & - \\
\hline & $\triangle \mathrm{AUR} 1$ & 12 & $1.81 \pm 0.10$ & $121 \%$ & $<0.0001$ & $0.69 \pm 0.09$ & $112 \%$ & 0.048 \\
\hline & $\triangle \mathrm{A} U \mathrm{R} 2$ & 13 & $1.76 \pm 0.09$ & $117 \%$ & $<0.0001$ & $0.70 \pm 0.05$ & $114 \%$ & 0.0029 \\
\hline
\end{tabular}

The animals were fed on $F$. graminearum for 5 weeks. F. candida, springtail Folsomia candida; T. tomentosa, isopod Trichorhina tomentosa; Rel. length, body length relative to animals fed on WT; Rel. width, body width relative to animals fed on WT. Size at the beginning of trial: $F$. candida length $0.48 \pm 0.08 \mathrm{~mm}$, width $0.11 \pm 0.02 \mathrm{~mm}$; T. tomentosa length $1.20 \pm 0.08 \mathrm{~mm}$, width $0.50 \pm 0.05 \mathrm{~mm}$. Length and width are shown as mean \pm s.d. $p$ values were determined using a two-tailed $t$ test. Source data are provided in a Source Data file

aurofusarin-containing mycelia corroborates the lack of toxicity of aurofusarin in springtails and isopods.

The low toxicity of aurofusarin in mealworms, Drosophila, isopods, and springtails could be accounted for by inefficient absorption, detoxification in the digestive tract, or fast clearance. Toxicity assays with cell cultures circumvent these effects, and we therefore investigated the effect of aurofusarin on a cell culture of the fall armyworm, Spodoptera frugiperda, which is an established toxicity model for insects ${ }^{14}$. Aurofusarin also exhibited relatively low toxicity in insect cells (Fig. 5c). The low toxicity of aurofusarin contradicts the hypothesis that fungal defence metabolites are toxic to predators ${ }^{12-15,19,20}$.

Mechanism of the induction of aurofusarin synthesis by grazing. Increased levels of aurofusarin in shaken cultures 
a

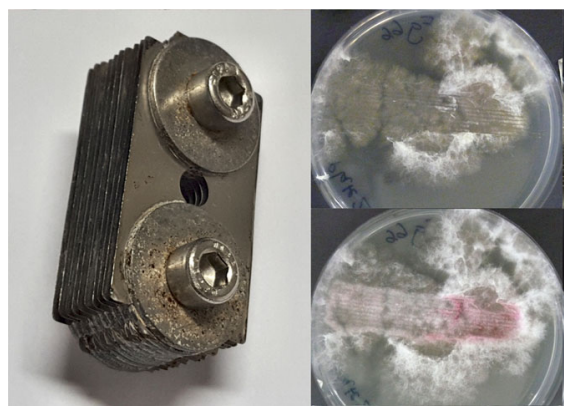

C

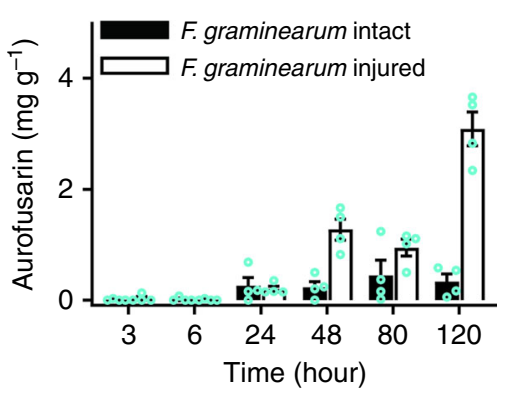

Fig. 6 Mechanical injury induces aurofusarin synthesis. a An array of 10 razor blades assembled at distances of 1 mm. b Fusarium graminearum IFA66 on GM7 medium at $22^{\circ} \mathrm{C}$ immediately after injury with the razor blade array (upper photo) and $24 \mathrm{~h}$ later (lower photo). c $F$. graminearum cultures were

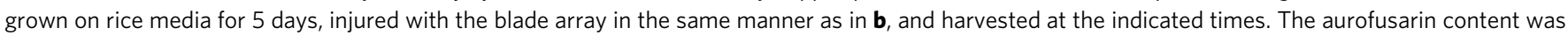
determined via HPLC-MS/MS ( $n=4$, error bars show s.e.m). Source data are provided in a Source Data file

(Fig. 2b) and in mycelia that had been exposed to a wide range of predators with different feeding modes (Fig. $2 \mathrm{~d}$, e, i-j) indicate that mechanical damage alone stimulates the synthesis of aurofusarin in F. graminearum. To test this hypothesis, we injured the mycelium of F. graminearum using an array of razor blades and monitored the aurofusarin content in the mycelia (Fig. 6). The results confirmed that mechanical damage was sufficient to induce aurofusarin synthesis in F. graminearum and showed that the effect was local (remaining confined to damaged parts of mycelia) and that the accumulation of increased levels of aurofusarin continued for at least $120 \mathrm{~h}$ after the injury. The mycelia of $F$. graminearum and F. culmorum in shaken liquid cultures accumulated more aurofusarin than still cultures, indicating that shaking caused mechanical injury (Supplementary Fig. 3). $F$. avenaceum and $F$. poae accumulated low amounts of aurofusarin in both culture types, but $F$. venenatum produced higher amounts of aurofusarin in still cultures than in shaken cultures, contradicting the results obtained with F. graminearum and F. culmorum. To clarify the discrepancy, the mycelium of $F$. venenatum that had been growing on an agar medium was injured with a razor blade array (Supplementary Fig. 6). Within $24 \mathrm{~h}$, the injured mycelia turned red, showing that mechanical damage induced aurofusarin synthesis also in F. venenatum. As shaking has not stimulated aurofusarin synthesis in $F$. venenatum (Supplementary Fig. 3), it apparently has not caused damage in this fungus comparable to cutting (Supplementary Fig. 6) or predation (Supplementary Fig. 4b).

Further fungal bis-naphthopyrones act as antifeedants. The magnitude of the deterrence effect of aurofusarin and the wide range of predators responsive to the antifeedant indicated that aurofusarin is F. gramineraum's major defence metabolite. Dimeric naphthopyrones similar to aurofusarin are produced by many genera of filamentous ascomycetes. Core structures of over 50 such metabolites are shown in Fig. 7. Their biological function is unknown. Viomellein and xanthomegnin-which are produced by many species of Aspergillus, Penicillium, Trichophyton, and other genera-were selected to investigate their inducibility by predation and their antifeedant activity towards arthropods. Induction of the viomellein synthesis by grazing was tested by subjecting cultures of the viomellein producer Aspergillus ochraceus to grazing by the springtail $F$. candida. The analysis of extracts of grazed and controlled fungal cultures by HPLC showed that grazing stimulated the synthesis of viomellein in fungal mycelia (Fig. 8a).

The deterrent effect of xanthomegnin and viomellein on the springtail F. candida was tested by offering baker's yeast spiked with these metabolites to the springtails in food choice experiments. At a spiking level of $10 \mathrm{mg} \mathrm{g}^{-1}$, the springtails strongly avoided yeast containing either xanthomegnin or viomellein (Fig. 8b, d). After 20 min, hardly any animal was found feeding on food spiked with xanthomegnin or viomellein. The deterrence was less prominent yet still highly significant at a spiking level of $2 \mathrm{mg} \mathrm{g}^{-1}$ (Fig. 8c, e). The results showed that the fungal bis-naphthopyrones viomellein and xanthomegnin are antifeedants that exert effects similar to aurofusarin on the springtail $F$. candida.

\section{Discussion}

Secondary metabolite synthesis in fungi is highly diverse, and most secondary metabolite pathways are species-specific ${ }^{32,33}$. Identical or similar structures are rarely found among secondary metabolites produced by more than two fungal genera. In contrast to other secondary metabolites, dimeric naphthopyrones have been found in all genera of filamentous ascomycetes investigated thus far, suggesting that they fulfil a common and widespread biological function. Our study indicates that this function is defence against animal predators.

Fungal strains defective in secondary metabolism owing to dysfunctional global regulator velvet complex ${ }^{20,34}$, which controls secondary metabolism and development ${ }^{35}$, and strains with constitutively stimulated secondary metabolism ${ }^{36}$ were used in food choice experiments. Predators preferred strains impaired in secondary metabolite synthesis and avoided strains with constitutively stimulated secondary metabolite synthesis, but the pleiotropic character of these mutations prevented identification of metabolites responsible for the effects. Mycotoxin sterigmatocystin was most often implicated in defence, but pathwayspecific mutants failed to confirm its role ${ }^{20}$. Our results indicate that the metabolites responsible for the loss of protection against predation in fungal strains with globally suppressed secondary metabolism were dimeric naphthopyrones.

If bis-naphthopyrones are defence metabolites ubiquitous among ascomycetes, why was the induction of their synthesis by predation not observed earlier? Transcriptomic studies in fungi have included various sorts of treatments, but surprisingly the effect of predation on fungal transcriptome has not been investigated. The effect of predation on fungal metabolome was addressed in a single study, in which Aspergillus nidulans was exposed to grazing by the springtail F. candida $a^{15}$. The metabolites found to be stimulated by predation did not include naphthopyrones. A. nidulans produces naphthopyrone YWA1, which is similar to the aurofusarin precursor rubrofusarin $^{28}$ and is dimerised into green pigment ${ }^{37}$ in the same way that rubrofusarin is dimerised into aurofusarin ${ }^{28}$. Why was the synthesis of the pigment not stimulated by grazing? The likely 


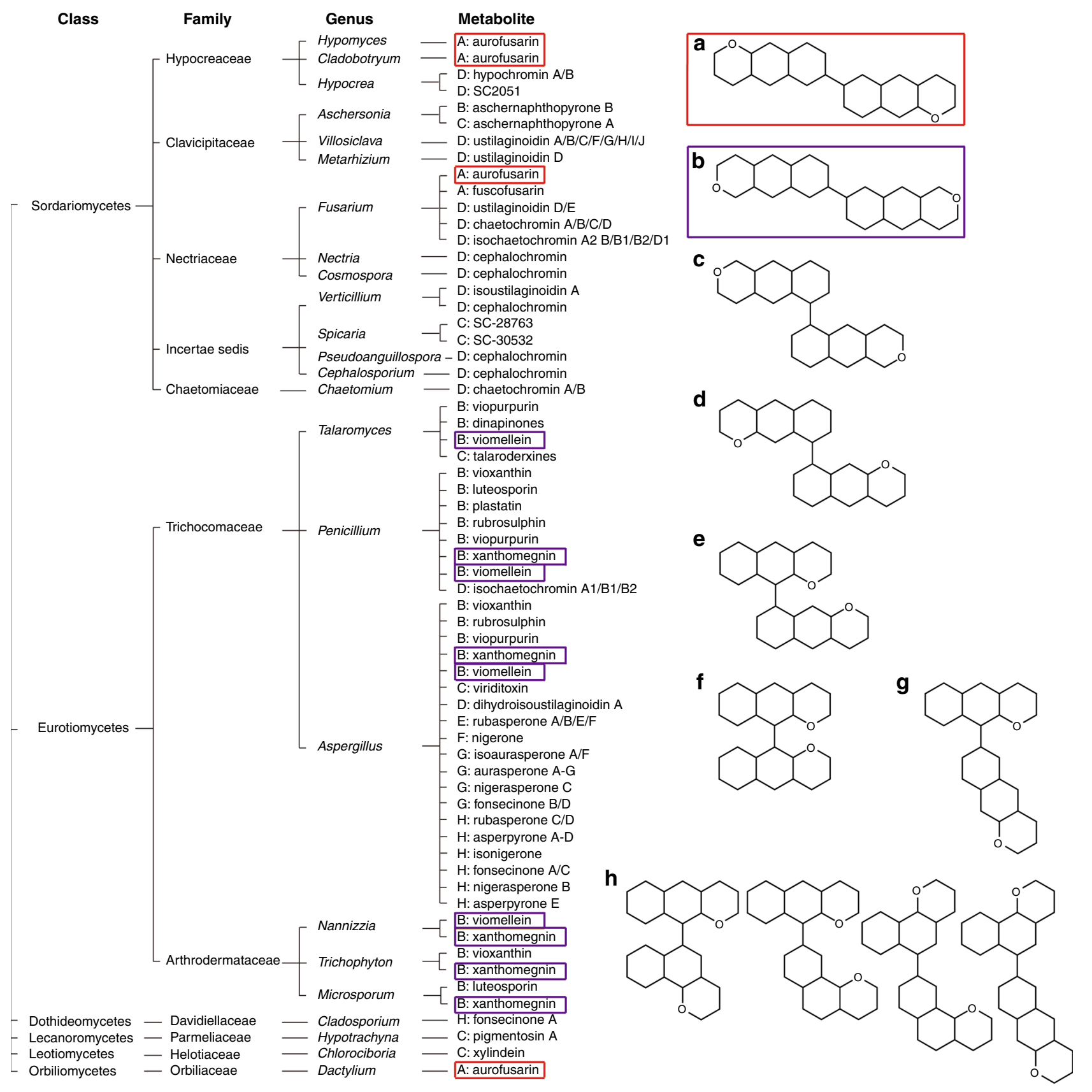

Fig. 7 Production of dimeric naphthopyrones by ascomycetous fungi. The taxonomic affiliation of selected fungal genera that produce dimeric naphthopyrones ${ }^{27}$ is shown on the left with schematic structures of bis-naphthopyrones on the right. The Structure Classes A to G contain dimeric naphtho- $\alpha$-pyrones and naphtho- $\gamma$-pyrones consisting of linear heptaketides; the classes differ by the position of the link between monomers and by the presence of either $\alpha$ - or $\gamma$-pyrones. The metabolites of Class $\mathrm{H}$ contain angular heptaketides, and all metabolites of this class listed here contain $\gamma$-pyrones. The metabolites used in this study have been labelled

reason was that as in most studies of interactions between A. nidulans and arthropods conducted thus far, in this study, an $A$. nidulans strain carrying mutation veA1 was used, which is defective in secondary metabolite production and defence respon$\mathrm{ses}^{38}$. VeA is part of the velvet complex, which is needed for aurofusarin synthesis in F. graminearum ${ }^{39}$. The metabolites induced by grazing in A. nidulans veA1 may belong to a second-tier level of defence, though our results indicate that in F. graminearum, bisnaphthopyrones are mainly-if not only-defence metabolites that target predators.

One Fusarium species that does not produce dimeric naphthopyrones is F. proliferatum. The absence of antifeedant metabolites in this species is in line with the observation that wheat kernels colonised with $F$. proliferatum attracted rather than repelled the mealworm Tenebrio molitor ${ }^{40}$. F. proliferatum survives a passage through the digestive system of $T$. molitor, and its propagules continue to be disseminated by faeces of the beetle long after ingestion ${ }^{41}$, suggesting that the loss of bisnaphthopyrone synthesis may have been selected in this species during its adaptation to dissemination by insects.

Aurofusarin synthesis in Fusarium species was induced by predators with different feeding modes and by cutting mycelia with razor blades, indicating that mechanical damage was sufficient to trigger chemical defence against predation. The potential 
a
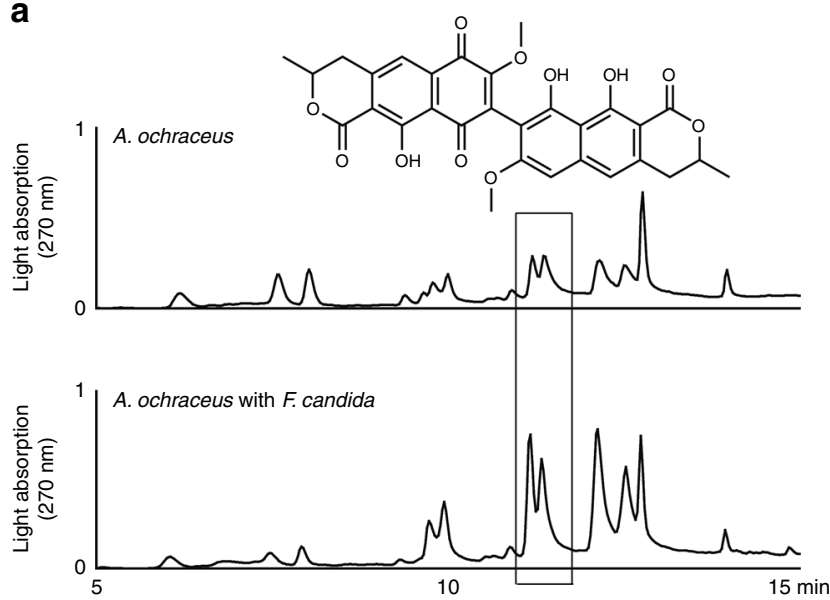

b

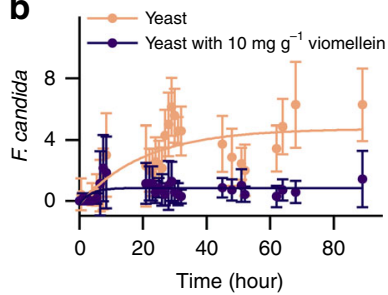

d

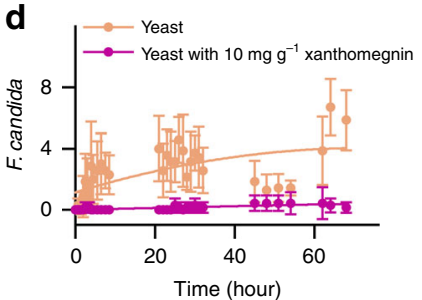

c

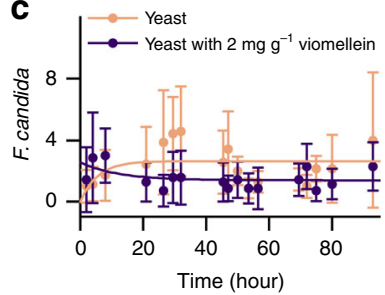

e

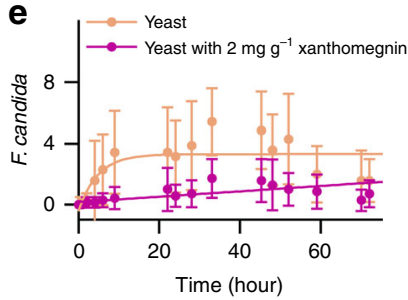

Fig. 8 Antifeedant activity of viomellein and xanthomegnin. a Aspergillus ochraceus was grown on rice kernels for 5 d. The springtail Folsomia candida was added to fungal cultures and allowed to feed for $6 \mathrm{~d}$. The cultures were extracted with chloroform/methanol (80:20) and analysed via HPLC with light absorption detection. Viomellein appeared as two peaks originating from stereoisomers (atropisomers) and separated owing to restricted intramolecular rotation around the biaryl axis. b-e The food preference of springtails Folsomia candida for yeast amended with viomellein or xanthomegnin. The animals were placed into the middle of arenas containing dry yeast on one side and dry yeast amended with viomellein or xanthomegnin on the other side. The number of springtails visiting each diet was recorded in a time series. Seven replicates were used, each with 20 animals. No statistical test was used for the data obtained for $10 \mathrm{mg} \mathrm{g}^{-1}$ of bis-naphthopyrones $\mathbf{b}$, d. For the data obtained with $2 \mathrm{mg} \mathrm{g}^{-1}$ of bis-naphthopyrones $\mathbf{c}$, e, cumulative values for all time points beginning with $20 \mathrm{~h}$ were compared using two-tailed $t$ tests. The preference for yeast without naphthopyrones was highly significant for both metabolites (viomellein: $n=14, p=0.0054$; xanthomegnin: $n=10, p<0.0001$ ). Error bars show $95 \% \mathrm{Cl}$. Source data are provided in a Source Data file

for the activation of defence responses by shaking or stirring fungal cultures in buffled flasks and fermenters should be considered in physiological studies on fungi in liquid media. The induction of bis-naphthopyrones synthesis in fungi by wounding contrasts with the chemical defence of land plants against herbivory, which requires specific signals in addition to wounding ${ }^{42}$. Defence response induction in plants by wounding alone would lead to frequent false alarms because plant shoots are often injured by being hit by solid objects in the wind and owing to passing animals, whereas fungal hyphae are protected by the solid substrates inside of which they grow. The second difference between the chemical defence of plants and fungi against predation is that plant defence against herbivory spreads systemically and even reaches neighbouring plants via volatile signals ${ }^{43}$, whereas fungal defence remains confined to the area affected by the damage (Fig. 6, Supplementary Figs. 4 and 6).

The presence of defence metabolites of the same structural class that presumably possess the same mode of action in many fungal species is likely to exert a strong selection pressure on the predators. If insects are notorious for their rapid development of resistance to insecticides ${ }^{44}$ and mushroom-feeding insects tolerate mushroom toxins ${ }^{18}$, why are bis-naphthopyrones still active against a wide range of fungivores that have likely been exposed to these metabolites for hundreds of millions of years? Aurofusarin content in F. graminearum that has been exposed to predation is very high (Figs. 2d, e). The maintenance of the high production of aurofusarin that may infer substantial fitness costs -as evidenced by markedly increased growth rates of mutants with disrupted aurofusarin synthesis ${ }^{25,29}$-must have been subjected to a strong selection pressure. We hypothesise that high levels of aurofusarin in fungal mycelia prevented predators from adaptation by saturating molecular targets of aurofusarin with binding affinities reduced by mutations, or by overwhelming enzymatic degradation. Mycotoxins never accumulate in comparably high concentrations, which would probably cause selfpoisoning that even protection mechanisms of mycotoxin producers ${ }^{45}$ could not prevent. The synthesis of an antifeedant of low toxicity in large amounts exemplifies a new concept in fungal chemical defence, with aurofusarin, viomellein, and xanthomegnin serving as the first examples. The ubiquity of bisnaphthopyrone pigments in ascomycetes indicates that this defence mechanism is widespread. The defence function of aurofusarin extends and modifies Janzen's 40-year-old hypothesis that fungi protect their substrates from animals via poisonous chemicals ${ }^{11}$. Rather than structurally diverse toxins produced in low amounts, structurally similar antifeedants accumulating at high amounts protect fungi from soil-dwelling predators. The intriguing question for future research is how a single metabolite class deters a wide range of predators. The presence of gustatory receptors triggered by bis-naphthopyrones in phylogenetically distant arthropods, including crustaceans, springtails, and insects, indicates that natural ligands of these receptors are compounds common in food substrates of all arthropods, such as proteins or polysaccharides.

\section{Methods}

Animals. The larvae of the beetle Tenebrio molitor (Insecta: Coleoptera) and the isopod Trichorhina tomentosa (Crustacea: Oniscidea) were purchased from Zoo \& Co. Zoo-Busch and b.t.b.e. Insektenzucht GmbH (Schnürpflingen, Germany). The culture of F. candida (strain: Berlin) was obtained from the Institute of Zoology, University of Goettingen, Germany, and was kept on Petri dishes filled with a layer of gypsum plaster with charcoal (9: 1). The culture of Porcellio scaber was initiated using animals collected in Göttingen, Germany, in spring 2017. The culture of Drosophila melanogaster was initiated with animals caught in 2006 in Kiel, Germany. Cultures of the nematodes Aphelenchoides saprophilus and Bursaphelenchus mucronatus were obtained from Prof. Liliane Ruess, Humboldt University of Berlin, Germany, and Professor Jiafu Hu, Zhejiang Agriculture and Forestry University, China, respectively.

Fungal strains. The F. graminearum strain IFA66 ${ }^{46}$ (DON chemotype) was obtained from Dr Marc Lemmens (BOKU, Tull, Austria) via Professor Thomas Miedaner (University of Hohenheim, Germany). Aurofusarin-deficient mutants were generated by disrupting the polyketide synthase gene PKS12 ${ }^{25}$ in F.graminearum 1003 and were labelled $\triangle$ AUR1, $\triangle$ AUR2, and $\triangle$ AUR3. The zearalenone-deficient mutant $\triangle \mathrm{ZEN}$ was generated from the same parent by disrupting the polyketide synthase gene PKS4 involved in zearalenone synthesis. The deoxynivalenol-deficient mutant $\triangle D O N$ was generated from the strain F. graminearum 3211 by disrupting Tri5 gene ${ }^{47}$. F. culmorum $3.37^{40}$ was a gift from late Professor Heinz-Wilhelm Dehne (University of Bonn, Germany). A. ochraceus 6692 was obtained from Professor Rolf Geisen, Max Rubner-Institut, Karlsruhe, 
Germany. F. avenaceum BBA92013 was obtained from Professor Tapani YliMattila, Turku, Finland. F. tricinctum RD30, F. venenatum RD15, and F. venenatum RD90 were isolated from weed samples in Germany and provided by $\mathrm{Dr}$ Raana Dastjerdi, University of Goettingen, Germany. F. poae DSMZ62376 was obtained from DSMZ (Braunschweig, Germany), and F. verticillioides M-8114 was obtained from the Fusarium Research Center (University Park, PA, USA). F. sporotrichioides IPP0249 was obtained from Prof. A. von Tiedemann (University of Goettingen, Göttingen, Germany).

Fungal media and cultures. The potato broth medium (PDB) was prepared by boiling $200 \mathrm{~g}$ of potatoes with the peel in $1 \mathrm{~L}$ of tap water for $20 \mathrm{~min}$ and autoclaving the filtrate with $20 \mathrm{~g}$ of glucose. GM7 medium ${ }^{48}$ contained $3 \mathrm{~g}$ L-asparagine, $1 \mathrm{~g} \mathrm{KH}_{2} \mathrm{PO}_{4}, 0.5 \mathrm{~g} \mathrm{MgSO}_{4} \cdot 7 \mathrm{H}_{2} \mathrm{O}$, 20 g glucose, $50 \mathrm{mg} \mathrm{CaCl}, 10 \mathrm{mg} \mathrm{FeCl} 3,1 \mathrm{mg}$ thiamine and $20 \mathrm{~g}$ agar in $1 \mathrm{~L} ; \mathrm{pH}$ was adjusted to 5.6. Liquid cultures of Fusarium spp. for the analysis of aurofusarin content were grown in $30 \mathrm{ml}$ of PDB in $100-\mathrm{ml}$ Erlenmeyer flasks at $23{ }^{\circ} \mathrm{C}$. Rice medium for the analysis of aurofusarin accumulation in Fusarium spp. cultures after injury with a razor blade array was prepared by autoclaving a mixture of $1.5 \mathrm{ml}$ of tap water with $0.5 \mathrm{~g}$ of rice powder (Alnatura $\mathrm{GmbH}$, Bickenbach, Germany) in glass Petri dishes with a diameter of $5 \mathrm{~cm}$. Rice medium for the investigation of the effect of predation on pigment accumulation was prepared by autoclaving $20 \mathrm{~g}$ of rice powder and $20 \mathrm{~g}$ of agar in $1 \mathrm{~L}$ of tap water and was poured into plastic Petri dishes with a diameter of $9 \mathrm{~cm}$.

\section{Purification of aurofusarin. Aurofusarin was extracted from $F$. graminearum} IFA66 that had been grown in PDB for 2 weeks at $25^{\circ} \mathrm{C}$ with shaking at $200 \mathrm{rpm}$. Fungal mycelium was freeze-dried, ground, and extracted with $50 \mathrm{ml}$ of chloroform/methanol (80:20) per gram of mycelium. The extract was cleared by centrifugation and the solvent removed in vacuum. Aurofusarin was purified by ethanol precipitation from phenol ${ }^{49}$ at $50^{\circ} \mathrm{C}$ followed by crystallisation from glacial acetic acid ${ }^{50}$. The purity of crystallised aurofusarin was verified by HPLC-ELSD (see "Aurofusarin analysis by HPLC-MS/MS and HPLC-ELSD").

Exposure of F. graminearum and F. culmorum to predation. For transcriptome analysis, 5000 fungal spores in $5 \mu \mathrm{L}$ of water were inoculated onto three rice kernels that had been autoclaved with $200 \mu \mathrm{l}$ of demineralised water (for arthropods) or onto $40 \mathrm{mg}$ of rice flour that had been autoclaved with $150 \mu \mathrm{l}$ of demineralised water (for nematodes) in $15-\mathrm{ml}$ Falcon tubes and incubated at $15^{\circ} \mathrm{C}$ in the dark. After $7 \mathrm{~d}$, the following predators were added to the fungal cultures: $20 \mathrm{mg}$ ( 200 individuals) of the springtail F. candida starved for 2 days or 2000-3000 individuals of the nematode Aphelenchoides saprophilus or 1000-2000 individuals of the nematode Bursaphelenchus mucronatus. Controls were incubated under the same conditions without animals. Each group consisted of four replicates.

Transcriptome analysis by RNAseq. F. graminearum IFA66 was exposed to predation by $F$. candida, as described above. After $48 \mathrm{~h}$, four cultures with predators and four control cultures were harvested, the animals were removed, and the total RNA was extracted using the RNAsnap method ${ }^{51}$, which was modified as follows: Fungal cultures were suspended in $400 \mu \mathrm{l}$ of RNA extraction solution (95\% deionised formamide, $18 \mathrm{~mm}$ EDTA, $0.025 \%$ SDS, and 1\% 2-mercaptoethanol) and disrupted via shaking with zirconia beads $(2.0 \mathrm{~mm}$ in diameter, Carl Roth, Karlsruhe, Germany) in the reciprocal mill MM 200 (Retsch, Haan, Germany) for 2 min at maximum power, followed by incubation at $95^{\circ} \mathrm{C}$ for $7 \mathrm{~min}$. Cell debris was removed by centrifugation at $16,000 \times g$ for $5 \mathrm{~min}$ at room temperature. The supernatant was transferred into an RNA precipitation mixture consisting of $800 \mu$ of isobutanol, $400 \mu \mathrm{l}$ of $5 \mathrm{~m}$ guanidine thiocyanate, and $5 \mu \mathrm{l}$ of linear polyacrylamide used as a co-precipitant (Co-Precipitant Pink, Bioline, London, UK). The mixture was centrifuged at $16,000 \times g$ for $5 \mathrm{~min}$ at room temperature, and the pellet was washed with $75 \%$ ethanol, dried, and dissolved in RNAase-free water.

Strand-specific cDNA libraries were prepared using Illumina's TruSeq stranded mRNA kit (75 bp paired-end) and sequenced on Illumina NextSeq 500V2. Data were analysed using the public server of Galaxy ${ }^{52}$ (https://usegalaxy.org/). Before the analysis, the reads were trimmed to remove low-quality sequences. The reads were mapped to the reference genome ( $F$. graminearum PH-1) using Hisat2 v2.0.5.1 with the default options for single-end data ${ }^{53}$. Cufflinks v2.2.1 ${ }^{54}$ was used to determine the abundance of transcripts in FPKM (Fragments Per Kilobase of exon per Million fragments mapped): The maximum intron length was set to 1000 nt, and the last annotated genome (ASM24013v3) was used as a reference. Cuffdiff v2.2.1.354 was used to determine the changes in gene expression compared with the control using an FDR (false discovery rate) of 0.05 . Only genes with a $\log _{2}$ FPKM (fold change) higher than 1.0 and a $q$ value lower than 0.01 were considered to have been significantly induced.

To identify upregulated secondary metabolite gene clusters, all upregulated genes were checked in the National Center for Biotechnology Information (NCBI) and in the European Bioinformatics Institute (EMBL-EBI) (UniProt) databases for signatures of polyketide synthetases (PKS), nonribosomal peptide synthetases, and terpenoid synthetases. Once more than $50 \%$ of the genes in a cluster and/or the signature enzyme of a cluster had been significantly induced (log2 FPKM (fold change) higher than 1.0 and $q$ value lower than 0.01 ), the literature related to the cluster was consulted to reveal the associated secondary metabolites. In this manner, gene clusters for the biosynthesis of aurofusarin ${ }^{55}$, C62 $62^{56,57}$, fusarin $\mathrm{C}^{56,58,59}$, fusaristatin $\mathrm{A}^{56,57}$, and terpenoids ${ }^{56}$ were identified. The results were corroborated with the help of AntiSMASH 3.060. The upregulated genes that had been located immediately before and after the signature enzymes were considered part of the cluster. Putative clusters without known signature enzymes were identified using AntiSMASH $3.0^{60}$ and corroborated manually.

Transcription analysis of aurofusarin pathway by RT qPCR. After exposure to F. candida grazing for $0,1,2,4,8,24,48,80$, and $120 \mathrm{~h}$ (F. graminearum) or 80 and $120 \mathrm{~h}$ (F. culmorum) and after nematode feeding for 24 and $80 \mathrm{~h}$ (F. graminearum), fungal mycelia were harvested, frozen in liquid nitrogen, and ground. RNA was extracted using the guanidinium thiocyanate-phenol-chloroform method ${ }^{61}$, precipitated with $4 \mathrm{M} \mathrm{LiCl}$ for $3 \mathrm{~h}$ on ice, and reverse transcribed with RevertAid Reverse Transcriptase (Thermo Fisher Scientific, California, USA) and random primers according to the manufacturer's instructions using $400 \mathrm{ng}$ of RNA in $20-\mu \mathrm{l}$ reactions. The cDNA obtained was used as a template for PCR, which contained ThermoPol Reaction Buffer (20 mm Tris-HCl, $10 \mathrm{~mm}\left(\mathrm{NH}_{4}\right)_{2} \mathrm{SO}_{4}, 10 \mathrm{~mm} \mathrm{KCl}, 0.1 \%$ Triton-X-100, $\mathrm{pH} 8.8$ at $25^{\circ} \mathrm{C}$ ) with $2.5-4.5 \mathrm{~mm} \mathrm{MgCl}, 200 \mu \mathrm{m}$ dNTP, $0.3 \mu \mathrm{m}$ forward and reverse primers (Supplementary Data 2), SYBR Green I (Invitrogen, Karlsruhe, Germany), $1 \mathrm{mg} \mathrm{ml}^{-1}$ bovine serum albumin, $0.03{\mathrm{U} \mu l^{-1}}^{\mathrm{T}}$ Taq polymerase (New England Biolabs, UK), and $1 \mu \mathrm{l} \mathrm{cDNA}$ as a template. PCR conditions were as follows: $95^{\circ} \mathrm{C}$ for $2 \mathrm{~min}, 35 \mathrm{cycles}$ of $94^{\circ} \mathrm{C}$ for $20-30 \mathrm{~s}, 59^{\circ} \mathrm{C}$ for $30-40 \mathrm{~s}$ and $68^{\circ} \mathrm{C}$ for $30 \mathrm{~s}$, with a final extension of $68^{\circ} \mathrm{C}$ for $15 \mathrm{~min}$ followed by a melting curve analysis beginning at $95^{\circ} \mathrm{C}$ with a decrement of $0.5-55^{\circ} \mathrm{C}$. GAPDH- (glyceraldehyde-3-phosphate dehydrogenase) and EFIA (elongation factor 1-alpha) genes were used as an internal reference, and the primers are shown in Supplementary Data 2. Three to eight biological replicates were analysed (Supplementary Data 3). The amplification efficiency for each gene obtained with the help of serial dilutions was used to calculate relative transcript levels (fold change) ${ }^{62}$. The significance of differences between cultures subjected to predators and controls was determined, as described in "Statistics and reproducibility".

Analysis of food preference. The preference of the springtail $F$. candida and the isopod Trichorhina tomentosa for fungal strains was studied on Petri dishes (92 $\mathrm{mm}$ in diameter) filled with a mixture of gypsum plaster and charcoal (9:1). Fungal cultures were grown on potato agar prepared from potato broth (see above) solidified with $15 \mathrm{~g} \mathrm{~L}^{-1}$ agar and kept at $25^{\circ} \mathrm{C}$ for $7-8 \mathrm{~d}$ in the dark. Agar plagues were cut from the edge of fungal colonies using a sterile cord borer (12 $\mathrm{mm}$ in diameter) and placed onto discs of matching size cut from Parafilm and placed on the opposite sides of the Petri dishes. Twenty F. candida individuals or eight Trichorhina tomentosa individuals that had been starved for 2 days were placed into the centre of the Petri dishes, and the plates were incubated at $15^{\circ} \mathrm{C}$ in the dark. The number of collembolans and isopods on each mycelium was recorded.

Food choice experiments with the mealworm T. molitor were carried out with fungal mycelia as well as with wheat flour amended with aurofusarin. In an experiment with fungal mycelia, F. graminearum strains were cultured on glass slides covered with PDA. After $7-8 \mathrm{~d}$ at $25^{\circ} \mathrm{C}$, the slides were placed on the opposite sides of Petri dishes ( $150 \mathrm{~mm}$ in diameter), and eight T. molitor larvae were placed in the middle of the plates. The number of animals visiting each fungal culture was recorded by taking photos at time intervals for $12 \mathrm{~h}$, and animals inside $13 \times 6-\mathrm{cm}$ rectangles that had been drawn around each slide were counted. The experiments with wheat flour were carried out with groups of animals and with single animals. Wheat flour (summer wheat variety Taifun) was amended with aurofusarin that had been dissolved in chloroform to reach a final concentration of $10 \mathrm{mg} \mathrm{g}^{-1}$. After shaking at room temperature for $1 \mathrm{~h}$, the chloroform was removed in a vacuum and the flour was left in an open Petri dish overnight in a fume hood. Flour samples used as a control were treated with pure chloroform in the same manner. Portions of $0.4 \mathrm{~g}$ of flour with and without aurofusarin were placed on the opposite sides of Petri dishes. Five larvae of T. molitor were added to each of three Petri dishes and allowed to feed for $4 \mathrm{~d}$ at room temperature and in ambient light. The animals were subsequently removed, and the weight of the remaining wheat flour with and without aurofusarin (mixed with faecal pellets left by the animals) was determined. The experiments with single animals were carried out with portions of $100 \mathrm{mg}$ of flour in Petri dishes covered with light-tight plant pot saucer trays. Single 3month-old mealworms $(23.9 \pm 1.8 \mathrm{~mm})$ were placed into the centre of 20 Petri dishes with one portion of flour with aurofusarin and another portion without aurofusarin. The light-blocking trays were opened for two seconds in dim light at fixed time intervals to record the mealworm location $(n=20)$.

Food choice experiments addressing the effect of xanthomegnin and viomellein on F. candida were carried out with dry yeast (Dr. August Oetker Nahrungsmittel KG, Bielefeld, Germany). The yeast was amended with aurofusarin dissolved in chloroform to achieve final concentrations of $2 \mathrm{mg} \mathrm{g}^{-1}$ and $10 \mathrm{mg} \mathrm{g}^{-1}$, chloroform was removed in vacuum and the yeast was left in open Petri dish overnight in a fume hood. Yeast for the controls was treated with pure chloroform. Twenty collembolans starved for 2 days were added to Petri dishes filled with charcoal/ plaster (1:9) and that contained $3 \mathrm{mg}$ of dry yeast on one side and $3 \mathrm{mg}$ of dry yeast amended with viomellein or xanthomegnin on the other side and the number of springtails visiting each diet was recorded in a time series. 
Toxicity of aurofusarin to mealworm Tenebrio molitor. Wheat flour was amended with aurofusarin dissolved in chloroform, and the solvent was evaporated, leading to aurofusarin concentrations of $0,1,10,100,1000$, and $10,000 \mu \mathrm{g} \mathrm{g}^{-1}$. Groups of ten larvae of T. molitor were weighed individually and placed in Petri dishes containing $1 \mathrm{~g}$ of wheat flour amended with different amounts of aurofusarin. After $10 \mathrm{~d}$ at $18^{\circ} \mathrm{C}$ in the dark, the weight of the animals was determined again. Each treatment consisted of four replicates, and two independent experiments were carried out.

Toxicity of aurofusarin to insect cells in tissue culture. The S. frugiperda 9 (Sf9) cell line was maintained in a Sf-900 II medium (Thermo Fisher Scientific China, Shanghai, China) and grown at $28^{\circ} \mathrm{C}$. The cells were seeded into 96 -well cell culture plates with $8 \times 10^{3}$ cells in $100 \mu \mathrm{l}$ medium per well, incubated for $24 \mathrm{~h}$ and treated with $100 \mu \mathrm{l}$ medium containing aurofusarin dissolved in dimethyl sulfoxide (DMSO) for $24 \mathrm{~h}$ with the final DMSO concentration not exceeding $0.1 \%$. MTT (3-(4,5-dimethylthiazol-2-yl)-2,5-diphenyltetrazolium bromide) solution $\left(0.5 \mathrm{mg} \mathrm{mL}^{-1}\right)$ was added to each well, and incubation was continued for $4 \mathrm{~h}$. Formazan precipitate was dissolved in $150 \mu \mathrm{L}$ of DMSO, and the absorbance of the solution at $490 \mathrm{~nm}$ was determined. Medium containing $0.1 \%$ DMSO was used as a negative control. The test was performed in triplicate.

Developmental toxicity of aurofusarin in Drosophila. Flies were reared at room temperature in BugDorm cages (MegaView Science, Taichung, Taiwan). Eggs were collected in Petri dishes left in the cages and incubated at $22^{\circ} \mathrm{C}$ in the dark for hatching. Larvae were transferred into 2-ml-Eppendorf tubes containing $160 \mathrm{mg}$ of medium, which were closed with sponge stoppers and incubated in a humid chamber in the dark at $25^{\circ} \mathrm{C}$. The standard food medium consisted of $62.5 \mathrm{~g}$ of baker's yeast, $62.5 \mathrm{~g}$ of cornmeal, $62.5 \mathrm{~g}$ of sucrose, and $12.5 \mathrm{~g}$ of agar per litre; the maize agar consisted of $188 \mathrm{~g}$ of cornmeal and $12.5 \mathrm{~g}$ agar per litre. The medium amended with aurofusarin was prepared by adding a chloroform solution of aurofusarin to a mixture of yeast, cornmeal, and sucrose, followed by removing the chloroform in a vacuum and adding the required amount of $1.25 \%$ agar in water autoclaved and cooled to $60^{\circ} \mathrm{C}$. After incubation on the medium with aurofusarin for 2 days (with the full medium and the cornmeal medium used as controls), all larvae were transferred to a fresh medium to accomplish the development, and the flies that emerged from pupae were counted daily.

Inducibility of aurofusarin synthesis by mechanical injury. To visualise aurofusarin induction by injury, fungal spores were spread onto GM7 medium incubated at $22^{\circ} \mathrm{C}$. The developed mycelia were injured with an array of 10 razor blades spaced at $1 \mathrm{~mm}$ apart. $24 \mathrm{~h}$ later, the aurofusarin accumulation was monitored as red pigment. For HPLC analysis of the aurofusarin accumulation after injury, 10,000 spores of $F$. graminearum IFA66 in $10 \mu$ of water were inoculated into the middle of rice medium in Petri dishes and incubated at $23^{\circ} \mathrm{C}$ in the dark for $5 \mathrm{~d}$. Mycelium on the plate surface was injured by five cuts with an array of 10 razor blades spaced at $1 \mathrm{~mm}$ apart. The cultures were kept at $23^{\circ} \mathrm{C}$, harvested at a time series, and extracted for aurofusarin analysis.

Exposure of Aspergillus ochraceus to predation. For treatment with the springtails, A. ochraceus 6692 was grown on three autoclaved rice kernels, as described for F. graminearum under "Exposure of F. graminearum and F. culmorum...". After $5 \mathrm{~d}$ at room temperature in the dark, the springtail $F$. candida was added $(20 \mathrm{mg}$, ca. 200 individuals) and allowed to feed for $6 \mathrm{~d}$. For treatments with mealworms, A. ochraceus was grown in a medium made by autoclaving $1 \mathrm{~g}$ of maize flour with $4 \mathrm{ml}$ of tap water in 100-ml Erlenmeyer flasks. After $5 \mathrm{~d}$ at room temperature in the dark, eight larvae of T. molitor were added to each flask and allowed to feed for $6 \mathrm{~d}$. The animals were removed, the cultures were freeze-dried, and viomellein was extracted with chloroform/methanol (80:20) using a 2-ml solvent for rice cultures and a $15-\mathrm{ml}$ solvent for maize cultures. The extracts were cleared by centrifugation and the solvent was removed in a vacuum. The residue was dissolved in DMSO.

Determination of aurofusarin and viomellein content by HPLC-DAD. Aurofusarin was extracted from freeze-dried cultures using chloroform/methanol $(80: 20)$. The extracts were cleared by centrifugation, the solvent was removed in a vacuum, and the residue was dissolved in DMSO. The aurofusarin content was determined by HPLC with a diode-array detector (DAD, Varian Prostar) using a polar-modified C18 column (Polaris Ether, $100 \times 2.0 \mathrm{~mm}$; Varian, Darmstadt, Germany) that was kept at $40{ }^{\circ} \mathrm{C}$, which was eluted with a gradient of Solvents A (water with $0.05 \%$ acetic acid and $5 \%$ acetonitrile) and B (methanol with $0.05 \%$ acetic acid): $0.1 \min 60 \%$ B, $11.9 \min 60-98 \%$ B, $2 \min 98 \%$ B, 1 min $98-60 \%$ B, $8 \mathrm{~min} 60 \% \mathrm{~B}$. Light absorption was monitored at $243 \mathrm{~nm}$. The aurofusarin standard turned out to be extremely unstable in protic solvents and under light, as has been observed by other researchers ${ }^{63}$. Stock solutions in DMSO were therefore kept at $-80^{\circ} \mathrm{C}$. We found that it was crucial to prepare calibration standards in pure methanol instead of using the mobile phase to prevent degradation during the run. Viomellein was extracted in the same manner as aurofusarin. The residue after solvent removal was dissolved in DMSO and analysed by HPLC on a C18 column (Kinetex $2.6 \mu \mathrm{m}, 50 \times 2.1 \mathrm{~mm}$, Phenomenex,
Aschaffenburg, Germany) that was kept at $40{ }^{\circ} \mathrm{C}$, which was eluted with a gradient of Solvents A (water with 3\% acetic acid and 5\% acetonitrile) and B (methanol with 3\% acetic acid) as follows: 2 min $40 \%$ B, 12 min 40-98\% B, 2 min $98-98 \%$, and $1 \mathrm{~min} 98-40 \%$ B, 8 min $40 \%$ B. Viomellein was detected by light absorption at $270 \mathrm{~nm}$. Aurofusarin and viomellein standards were purchased from Bioviotica (Göttingen, Germany).

Aurofusarin analysis by HPLC-MS/MS and HPLC-ELSD. Because the concentrations of aurofusarin in fungal mycelia that were determined by HPLC-DAD turned out to be unprecedentedly high, two other methods were used for confirmation. The first method was HPLC-MS/MS with separation on a polar-modified C18 column (Polaris Ether, $100 \times 2.0 \mathrm{~mm}$; Varian, Darmstadt, Germany) that was kept at $40^{\circ} \mathrm{C}$, which was eluted with a gradient of Solvents A (water with $0.1 \%$ formic acid) and B (methanol) as follows: $0.2 \min 40 \%$ B, 6.8 min $40-98 \%$ B, 2 min $98 \%$ B, 1 min $98-40 \%$ B, 5 min $40 \%$ B. The eluent was ionised by electrospray in a positive mode with a capillary voltage of $4000 \mathrm{~V}$. The triple quadrupole 6460 (Agilent, Darmstadt, Germany) that was used as a detector was operated in a selected reaction monitoring mode with the transition $\mathrm{m} / \mathrm{z}$ 571.3- $>556.0$. Aurofusarin standards were prepared shortly before analysis by diluting a stock solution in DMSO with pure methanol (see previous paragraph). As an additional verification method independent of the aurofusarin standard, HPLC coupled with an evaporative light scattering detector (ELSD) was used. The analyte was chromatographically separated (as described above), and the eluent was directed into the ELSD 1260 detector (Agilent, Darmstadt, Germany) operated at a nebuliser temperature of $40^{\circ} \mathrm{C}$, an evaporator temperature of $42^{\circ} \mathrm{C}$, and an evaporator gas flow of 1.6 standard litres per minute. Tetracycline hydrochloride (Sigma-Aldrich, Munich, Germany) was used as a standard.

Statistics and reproducibility. The investigators were not blinded. In the RNAseq experiment, four biological replicates were used because we were interested in the gene clusters that were most strongly induced. In the food choice experiments, the number of animals per arena was limited to 8-20 (depending on the species) due to the requirement that the animals not consume all of the food before the end of the experiment. Statistical tests were deemed unnecessary in certain food choice experiments (Fig. 3, Supplementary Fig. 5) and in the stimulation of aurofusarin synthesis by grazing (Fig. 2d, e, Fig. 6c) owing to the high magnitude of the effects. In time series shown in Fig. 3, Fig. 4b, Fig. 5b, Fig. 8b-e, and Supplementary Fig. 5 the same samples were measured repeatedly. In all other experiments each measurement was taken from a distinct sample.

Bar graphs show means \pm SEM, and line graphs show means $\pm 95 \%$ CI. Box plots show medians and lower and quartiles (Q1 and Q3), with whiskers showing the largest (smallest) observation or 1.5-fold of the interquartile range, whichever is smaller (larger) $(\mathrm{Q} 1-1.5 \times(\mathrm{Q} 3-\mathrm{Q} 1), \mathrm{Q} 3+1.5 \times(\mathrm{Q} 3-\mathrm{Q} 1)$. The significance of the differences between the means for the weight gain of mealworms and for the consumption of wheat flour with/without aurofusarin was tested via unpaired $t$ tests. The significance of differences in the transcription of the aurofusarin gene cluster was determined as follows: Ct values for target genes and two internal reference genes (glyceraldehyde-3-phosphate dehydrogenase and elongation factor 1a) were adjusted for amplification efficiencies, which were obtained from dilution series $^{62} . \Delta \mathrm{Ct}$ was calculated for predated cultures and controls, and the two groups were compared by unpaired two-tailed $t$ tests. The induction of gene expression was regarded as significant when relative mRNA levels of at least three genes increased at least threefold and the increase was statistically significant with both reference genes $\left({ }^{\star} 0.05<P<0.01,{ }^{*} 0.01<P<0.0001,{ }^{* *} P<0.0001\right)$. Sample size and $p$ values are shown in Supplementary Data File 3.

Reporting summary. Further information on research design is available in the Nature Research Reporting Summary linked to this article.

\section{Data availability}

RNAseq data are accessible at ArrayExpress under accession code E-MTAB-6939 [https://www.ebi.ac.uk/arrayexpress/experiments/E-MTAB-6939/] and their analysis is shown in Supplementary Data 1. The authors declare that all other data supporting the findings of this study are available within the article and its Supplementary Information, Supplementary Data files and in the Source Data file.

Received: 13 June 2018 Accepted: 5 July 2019

Published online: 08 August 2019

\section{References}

1. Lavelle, P., \& Spain, A. V. Regulation of mineralisation by invertebrates. In: Soil Ecology, pp. 411-414 (Kluyver Academic Publisher, New York, 2003).

2. Johnson, D. et al. Soil invertebrates disrupt carbon flow through fungal networks. Science 309, 1047 (2005).

3. Boddy, L., Wood, J., Redman, E., Hynes, J. \& Fricker, M. D. Fungal network responses to grazing. Fungal Genet. Biol. 47, 522-530 (2010). 
4. McGonigle, T. P. Effects of animals grazing on fungi. In: The Mycota IV: Environmental and Microbial Relationships, pp. 201-212 (Springer, Berlin, Heidelberg, 2007).

5. A'Bear, A. D., Jones, T. H. \& Boddy, L. Size matters: what have we learnt from microcosm studies of decomposer fungus-invertebrate interactions? Soil Biol. Biochem. 78, 274-283 (2014)

6. Crowther, T. W., Boddy, L. \& Hefin Jones, T. Functional and ecological consequences of saprotrophic fungus-grazer interactions. ISME J. 6, 1992-2001 (2012).

7. Brandhorst, T., Dowd, P. F. \& Kenealy, W. R. The ribosome-inactivating protein restrictocin deters insect feeding on Aspergillus restrictus. Microbiology 142, 1551-1556 (1996).

8. Avanzo, P. et al. Trypsin-specific inhibitors from the basidiomycete Clitocybe nebularis with regulatory and defensive functions. Microbiology 155, 3971-3981 (2009).

9. Wohlschlager, T. et al. Methylated glycans as conserved targets of animal and fungal innate defense. Proc. Natl. Acad. Sci. USA 111, 2787-2796 (2014).

10. Künzler, M. Hitting the sweet spot-glycans as targets of fungal defense effector proteins. Molecules 20, 8144-8167 (2015).

11. Janzen, D. H. Why fruits rot, seeds mold, and meat spoils. Am. Nat. 111, 691-713 (1977).

12. Wicklow, D. T. Metabolites in the coevolution of fungal chemical defence systems. In: Coevolution of Fungi with Plants and Animals, pp. 173-201 (Eds Pyrozinski, K. A. \& Hawksworth, D. L.), (Academic Press, London, 1988).

13. Dowd, P. F., Miller, J. D. \& Greenhalgh, R. Toxicity and interactions of some Fusarium graminearum metabolites to caterpillars. Mycologia 81, 646-650 (1989).

14. Dowd, P. F. Insect interaction with mycotoxin-producing fungi and their hosts. In Handbook of applied mycology Volume 4, pp. 137-155 (Eds Bhatnagar, D., Lillehoj, E. B. \& Arora, D. K.) (Marcel Dekker, New York, 1992).

15. Döll, K., Chatterjee, S., Scheu, S., Karlovsky, P. \& Rohlfs, M. Fungal metabolic plasticity and sexual development mediate induced resistance to arthropod fungivory. Proc. Biol. Sci. B 280, 1219 (2013).

16. Brandt, P., García-Altares, M., Nett, M., Hertweck, C. \& Hoffmeister, D. Induced chemical defense of a mushroom by a double-bond-shifting polyene synthase. Angew. Chem. Int. Ed. 56, 5937-5941 (2017).

17. Calvo, A. M. \& Cary, J. W. Association of fungal secondary metabolism and sclerotial biology. Front. Microbiol. 6, 62 (2015).

18. Jaenike, J., Grimaldi, D., Sluder, A. E. \& Greenleaf, A. L. Alpha-amanitin tolerance in mycophagous Drosophila. Science 221, 165-167 (1983).

19. Ruess L., Lussenhop J. (2005) Trophic Interactions of Fungi and Animals. In: The Fungal Community, 3rd edition, pp. 581-598. (Eds Dighton, J., White, J. F. \& Oudemans. P.) (Taylor \& Francis, Boca Raton 2005).

20. Rohlfs, M. Fungal secondary metabolite dynamics in fungus-grazer interactions: novel insights and unanswered questions. Front. Microbiol. 5, 788 (2015).

21. Hillmann, F. et al. Virulence determinants of the human pathogenic fungus Aspergillus fumigatus protect against soil amoeba predation. Environ. Microbiol. 17, 2858-2869 (2015).

22. Cary, J. W. et al. Functional characterization of a veA-dependent polyketide synthase gene in Aspergillus flavus necessary for the synthesis of asparasone, a sclerotium-specific pigment. Fungal Genet. Biol. 64, 25-35 (2014).

23. Zhao, Y. et al. Production of a fungal furocoumarin by a polyketide synthase gene cluster confers the chemo-resistance of Neurospora crassa to the predation by fungivorous arthropods. Environ. Microbiol. 19,3920-3929 (2017).

24. Ottmann, C. et al. A common toxin fold mediates microbial attack and plant defense. Proc. Natl. Acad. Sci. USA 106, 10359-10364 (2009).

25. Malz, S. et al. Identification of a gene cluster responsible for the biosynthesis of aurofusarin in the Fusarium graminearum species complex. Fungal Genet. Biol. 42, 420-433 (2005).

26. Põldmaa, K. Tropical species of Cladobotryum and Hypomyces producing red pigments. Stud. Mycol. 68, 1-34 (2011).

27. Donner, C. D. Naphthopyranones-isolation, bioactivity, biosynthesis and synthesis. Nat. Prod. Rep. 32, 578-604 (2015).

28. Frandsen, R. J. N. et al. Two novel classes of enzymes are required for the biosynthesis of aurofusarin in Fusarium graminearum. J. Biol. Chem. 286, 10419-10428 (2011).

29. Jung, S. Y., Kim, J. E., Yun, S. H. \& Lee, Y. W. Possible negative effect of pigmentation on biosynthesis of polyketide mycotoxin zearalenone in Gibberella zeae. J. Microbiol. Biotechnol. 16, 1392-1398 (2006).

30. Dvorska, J. E., Surai, P. F., Speake, B. K. \& Sparks, N. H. Effect of the mycotoxin aurofusarin on the antioxidant composition and fatty acid profile of quail eggs. Br. Poult. Sci. 42, 643-649 (2001).

31. Gudauskas, R. T., Davis, N. D. \& Diener, U. L. Sensitivity of Heliothis virescens larvae to aflatoxin in ad libitum feeding. J. Invertebr. Pathol. 9, 132-133 (1967).
32. Keller, N. P., Turner, G. \& Bennett, J. W. Fungal secondary metabolism - from biochemistry to genomics. Nat. Rev. Microbiol. 3, 937-947 (2005).

33. Lind, A. L. et al. Examining the evolution of the regulatory circuit controlling secondary metabolism and development in the fungal genus Aspergillus. PLoS Genet. 11,e1005096 (2015).

34. Rohlfs, M., Albert, M., Keller, N. P. \& Kempken, F. Secondary chemicals protect mould from fungivory. Biol. Lett. 3, 523-525 (2007).

35. Bayram, O. \& Braus, G. H. Coordination of secondary metabolism and development in fungi: the velvet family of regulatory proteins. FEMS Microbiol. Rev. 36, 1-24 (2012).

36. Yin, W.-B. et al. An Aspergillus nidulans bZIP response pathway hardwired for defensive secondary metabolism operates through aflR. Mol. Microbiol. 83, 1024-1034 (2012).

37. Klejnstrup, M. L. et al. Genetics of polyketide metabolism in Aspergillus nidulans. Metabolites 2, 100-133 (2012).

38. Regulin, A. \& Kempken, F. Fungal genotype determines survival of Drosophila melanogaster when competing with Aspergillus nidulans. PLoS ONE. 13, e0190543 (2018).

39. Merhej, J. et al. The velvet gene, FgVe1, affects fungal development and positively regulates trichothecene biosynthesis and pathogenicity in Fusarium graminearum. Mol. Plant Pathol. 13, 363-374 (2012).

40. Guo, Z. Q. et al. Effect of fungal colonization of wheat grains with Fusarium spp. on food choice, weight gain and mortality of meal beetle larvae (Tenebrio molitor). PLoS ONE. 9, e100112 (2014).

41. Guo, Z., Pfohl, K., Karlovsky, P., Dehne, H.-W. \& Altincicek, B. Dissemination of Fusarium proliferatum by mealworm beetle Tenebrio molitor. PLoS ONE. 13, e0204602 (2018).

42. Korth, K. L. \& Dixon, R. A. Evidence for chewing insect-specific molecular events distinct from a general wound response in leaves. Plant Physiol. 115, 1299-1305 (1997)

43. Baldwin, I. T., Halitschke, R., Paschold, A., von Dahl, C. C. \& Preston, C. A Volatile signaling in plant-plant interactions: 'talking trees' in the genomics era. Science 311, 812-815 (2006).

44. Scott, J. G. Cytochromes P450 and insecticide resistance. Insect Biochem. Mol. Biol. 29, 757-777 (1999).

45. Keller, N. P. Translating biosynthetic gene clusters into fungal armor and weaponry. Nat. Chem. Biol. 11, 671-677 (2015).

46. Miedaner, T. et al. Aggressiveness and mycotoxin production of eight isolates each of Fusarium graminearum and Fusarium verticillioides for ear rot on susceptible and resistant early maize inbred lines. Eur. J. Plant Pathol. 127, 113-123 (2010).

47. Maier, F. J. et al. Involvement of trichothecenes in fusarioses of wheat, barley and maize evaluated by gene disruption of the trichodiene synthase (Tri5) gene in three field isolates of different chemotype and virulence. Mol. Plant Pathol. 7, 449-461 (2006).

48. Utermark, J. \& Karlovsky, P. Genetic transformation of filamentous fungi by Agrobacterium tumefaciens. Protocol Exchange https://doi.org/10.1038/ nprot.2008.83 (2008).

49. Gray, J. S., Martin, G. C. J. \& Rigby, W. Aurofusarin. J. Chem. Soc. Chem. 1967, 2580-2587 (1967)

50. Ashley, J. N., Hobbs, B. C. \& Raistrick, H. Studies in the biochemistry of micro-organisms: the crystalline colouring matters of Fusarium culmorum (W. G. Smith) Sacc. and related forms. Biochem. J. 31, 385-397 (1937).

51. Stead, M. B. et al. RNAsnap: a rapid, quantitative and in excensive, method for isolating total RNA from bacteria. Nucleic Acids Res. 20, e156 (2012).

52. Afgan, E. et al. The galaxy platform for accessible, reproducible and collaborative biomedical analyses: 2018 update. Nucleic Acids Res. 8, W537-W544 (2018).

53. Kim, D., Langmead, B. \& Salzberg, S. L. HISAT: a fast spliced aligner with low memory requirements. Nat. Methods 12, 357-360 (2015).

54. Trapnell, C. et al. Transcript assembly and quantification by RNA-Seq reveals unannotated transcripts and isoform switching during cell differentiation. Nat. Biotechnol. 28, 511-515 (2010).

55. Frandsen, R. J. N. et al. The biosynthetic pathway for aurofusarin in Fusarium graminearum reveals a close link between the naphthoquinones and naphthopyrones. Mol. Microbiol. 61, 1069-1080 (2006).

56. Sieber, C. M. K. et al. The Fusarium graminearum genome reveals more secondary metabolite gene clusters and hints of horizontal gene transfer. PLoS ONE. 9, e110311 (2014)

57. Harris, L. J., Balcerzak, M., Johnston, A., Schneiderman, D. \& Ouellet, T. Host-preferential Fusarium graminearum gene expression during infection of wheat, barley, and maize. Fungal Biol. 120, 111-123 (2016).

58. Connolly, L. R., Smith, K. M. \& Freitag, M. The Fusarium graminearum histone H3 K27 methyltransferase KMT6 regulates development and expression of secondary metabolite gene clusters. PLoS Genet. 9,e1003916 (2013).

59. Niehaus, E.-M. et al. Genetic manipulation of the Fusarium fujikuroi fusarin gene cluster yields insight into the complex regulation and fusarin biosynthetic pathway. Chem. Biol. 20, 1055-1066 (2013). 
60. Weber, T. et al. antiSMASH 3.0-a comprehensive resource for the genome mining of biosynthetic gene clusters. Nucleic Acids Res. 43, W237-W243 (2015).

61. Chomczynski, P. \& Sacchi, N. The single-step method of RNA isolation by acid guanidinium thiocyanate-phenol-chloroform extraction: twentysomething years on. Nat. Protoc. 1, 581-585 (2006).

62. Pfaffl, M. W. A new mathematical model for relative quantification in realtime RT-PCR. Nucleic Acids Res. 29, e45 (2001).

63. Jarolim, K. et al. The secondary Fusarium metabolite aurofusarin induces oxidative stress, cytotoxicity and genotoxicity in human colon cells. Toxicol. Lett. 284, 170-183 (2018).

\section{Acknowledgements}

We thank R. Pilot (University of Goettingen) for her excellent technical assistance, F. Gremmes (University of Goettingen) for the construction of a cutting tool, J. Straus (BOKU Vienna, Austria) for advice on experiments with flour, and a referee for pointing out that high ligand concentrations may saturate low-affinity receptors. We are obliged to L. Ruess (Humboldt University Berlin, Germany), A. von Tiedemann (University of Göttingen, Germany), J. Hu (Zhejiang Agriculture and Forestry University, China), T. Miedaner (University of Hohenheim, Germany), H.-W. Dehne (University of Bonn, Germany), R. Geisen (Max-Rubner-Institute, Germany), T. Yli-Mattila (Turku University, Finland), and R. Dastjerdi (Seed and Plant Improvement Institute, Karaj, Iran) for providing us with fungal strains and invertebrate cultures and B.J. van Rensburg (ARC South Africa) for a photograph of moulded maize ear. This work was supported by the German Academic Exchange Service; the German Research Foundation (DFG IRTG 2172); the National Natural Science Foundation of China (21876152); the China Scholarship Concil; and the Ministry for Science and Culture of Lower Saxony, Germany.

\section{Author contributions}

P.K. conceived of and guided the study and wrote the manuscript. Y.X. carried out the interaction experiments as well as the RT qPCR- and HPLC-DAD analysis. M.V. carried out RNAseq analysis, and A.A. purified the aurofusarin and carried out the HPLC-MSand HPLC-ELSD analysis. L.S. carried out the feeding experiments and RT qPCR with nematodes, K.P. developed and guided the HPLC analysis, and M.R. provided conceptual support for experiments with animals. W.S. generated fungal strains with disrupted biosynthetic pathways, and W.C. carried out the cell culture experiments and provided guidance. All authors approved the manuscript.

\section{Additional information}

Supplementary Information accompanies this paper at https://doi.org/10.1038/s41467019-11377-5.

Competing interests: The authors declare no competing interests.

Reprints and permission information is available online at http://npg.nature.com/ reprintsandpermissions/

Peer review information: Nature Communications thanks the anonymous reviewers for their contribution to the peer review of this work. Peer reviewer reports are available.

Publisher's note: Springer Nature remains neutral with regard to jurisdictional claims in published maps and institutional affiliations.

cc (i) Open Access This article is licensed under a Creative Commons Attribution 4.0 International License, which permits use, sharing, adaptation, distribution and reproduction in any medium or format, as long as you give appropriate credit to the original author(s) and the source, provide a link to the Creative Commons license, and indicate if changes were made. The images or other third party material in this article are included in the article's Creative Commons license, unless indicated otherwise in a credit line to the material. If material is not included in the article's Creative Commons license and your intended use is not permitted by statutory regulation or exceeds the permitted use, you will need to obtain permission directly from the copyright holder. To view a copy of this license, visit http://creativecommons.org/ licenses/by/4.0/.

(c) The Author(s) 2019 


\section{SUPPLEMENTARY INFORMATION}

Protection of filamentous fungi from wide-range of predators by bis-naphthopyrone pigments

Xu et al. 

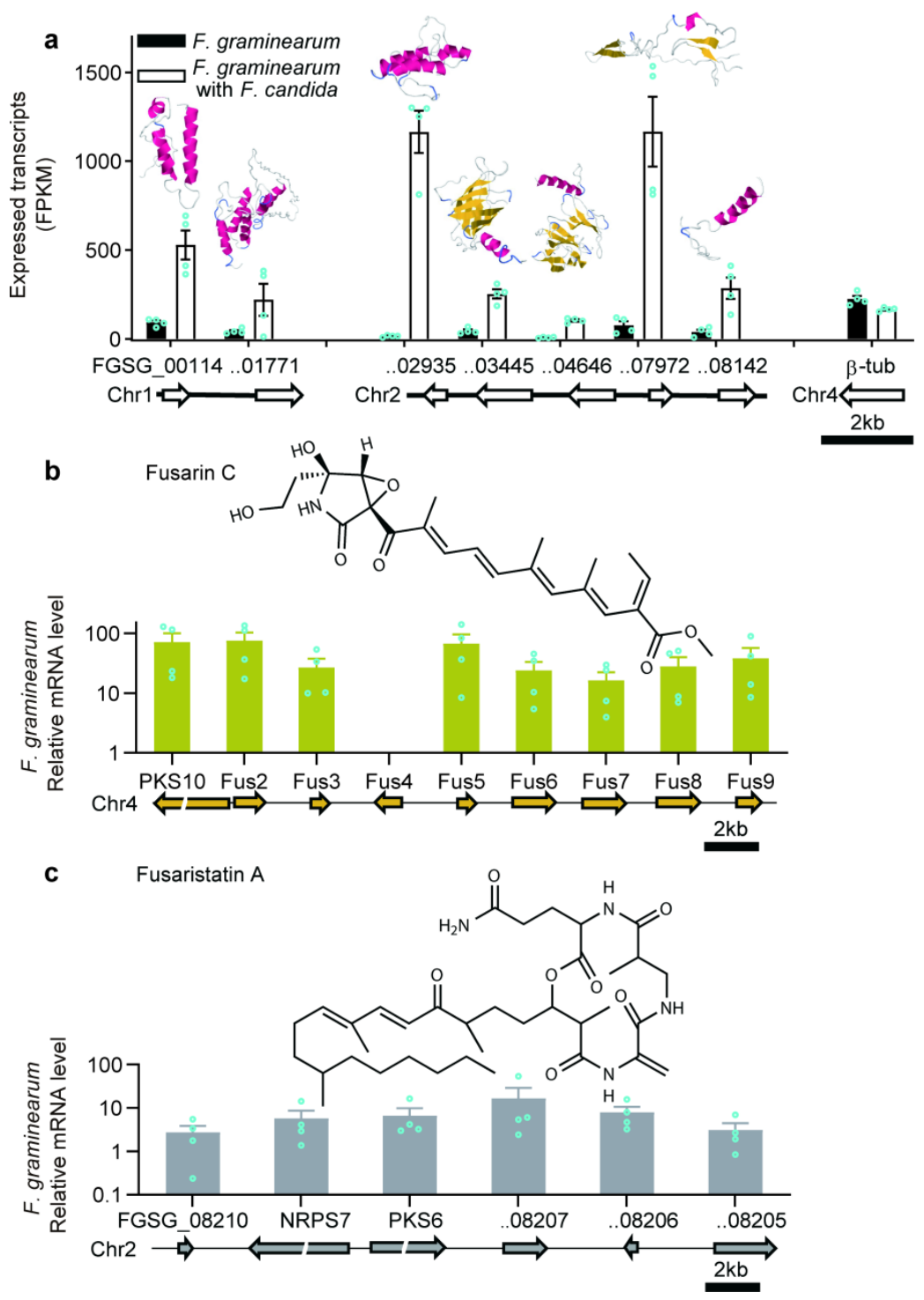

Supplementary Fig. 1 Springtails grazing induces biosynthetic pathways for cysteine-rich proteins, fusarin $\mathrm{C}$ and fusaristatin $\mathrm{A}$ in $F$. graminearum. a Fragments per kilobase of mapped reads (FPKM) generated by Cufflinks for 7 most strongly up-regulated genes encoding small secreted cysteine-rich proteins in $F$. graminearum when predated by the springtail $F$. candida for $48 \mathrm{~h}$ according to RNAseq data; beta-tubulin gene is shown as a reference. b,c Up-regulation of fusarin $\mathrm{C}$ and fusaristatin A pathways in $F$. graminearum after exposure to the springtail F. candida for $48 \mathrm{~h}$. The RNAseq analysis was carried out with four biological replicates; error bars show SEM. Source data are provided in a Source Data file. 


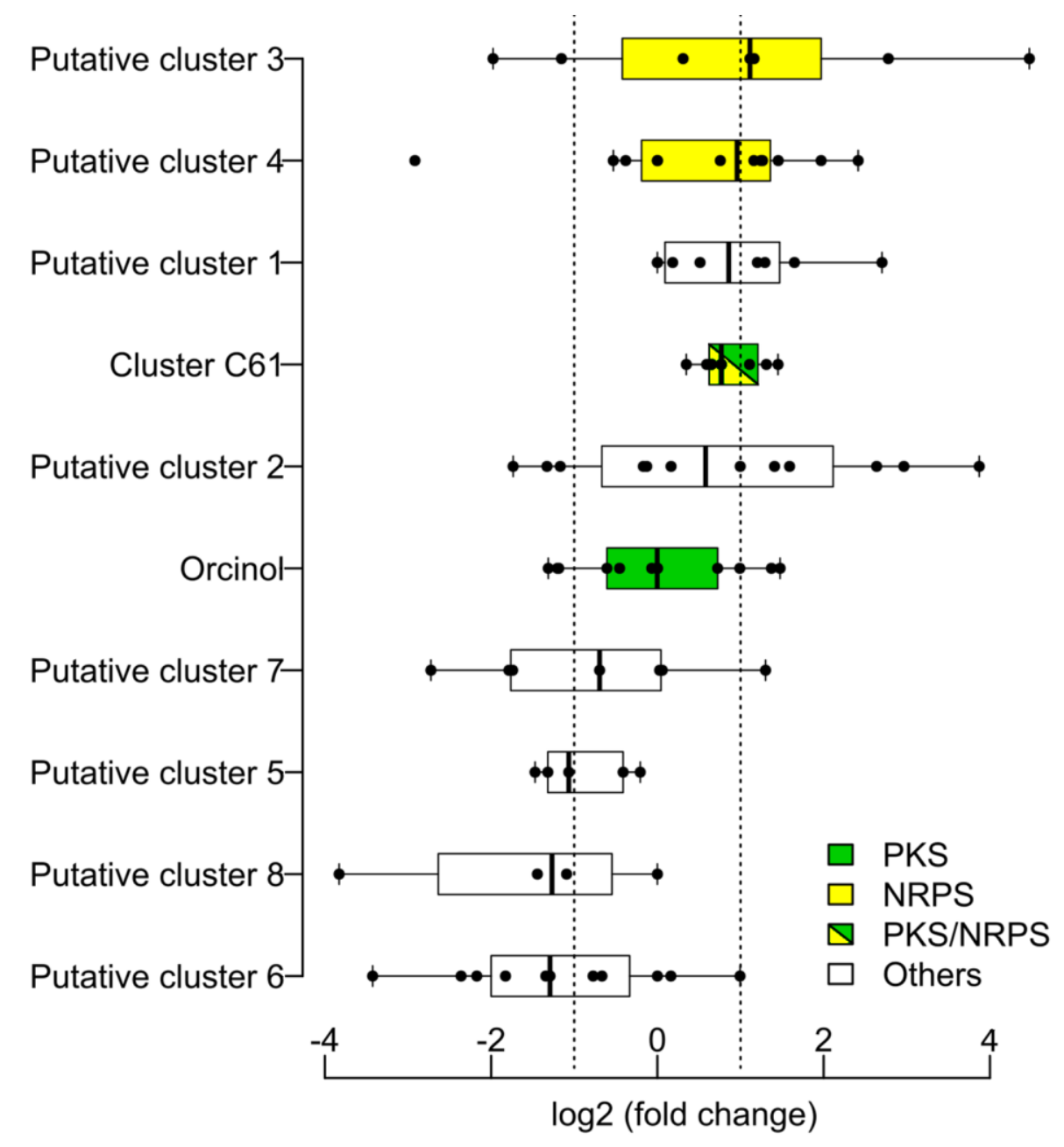

Supplementary Fig. 2 Effect of springtail grazing on the transcription of additional gene clusters encoding secondary metabolite synthesis in Fusarium graminearum. F. graminearum was exposed to the springtail $F$. candida for $48 \mathrm{~h}$ and mRNA levels were determined by RNAseq. Accession numbers: putative cluster 3 (FGSG_03428 to FGSG_03434), putative cluster 4 (FGSG_07820 to FGSG_07831) putative cluster 1 (FGSG_01672, FGSG_01673, FGSG_01675 to FGSG_01677, FGSG_01679 to FGSG_01681), cluster C61 (FGSG_10542, FGSG_10543, FGSG_10545 to FGSG_10547, FGSG_10549, FGSG_13782), putative cluster 2 (FGSG_11984 to FGSG_11989, FGSG_01685 to FGSG_01690), orcinol (FGSG_03956 to FGSG_03959, FGSG_03962 to FGSG_03971), putative cluster 7 (FGSG_09060 to FGSG_09066), putative cluster 5 (FGSG_04173 to FGSG_04177), putative cluster 8 (FGSG_10494 to FGSG_10496, 
FGSG_10498), putative cluster 6 (FGSG_04740 to FGSG_04750). The RNAseq analysis was carried out on four biological replicates. Box plots show the median for each cluster and lower and upper quartiles (Q1 and Q3). Up-regulated clusters were defined as having $>50 \%$ of the genes and/or the gene encoding the signatures enzyme induced [log2 FPKM (fold change) higher than 1.0 (dotted line) and a q value lower than 0.01]. Whiskers show the largest (smallest) observation or 1.5-fold of the interquartile range, whichever is smaller (larger) [Q1-1.5*(Q3-Q1) and $\mathrm{Q} 3+1.5 *(\mathrm{Q} 3-\mathrm{Q} 1)]$. Source data are provided in a Source Data file. 


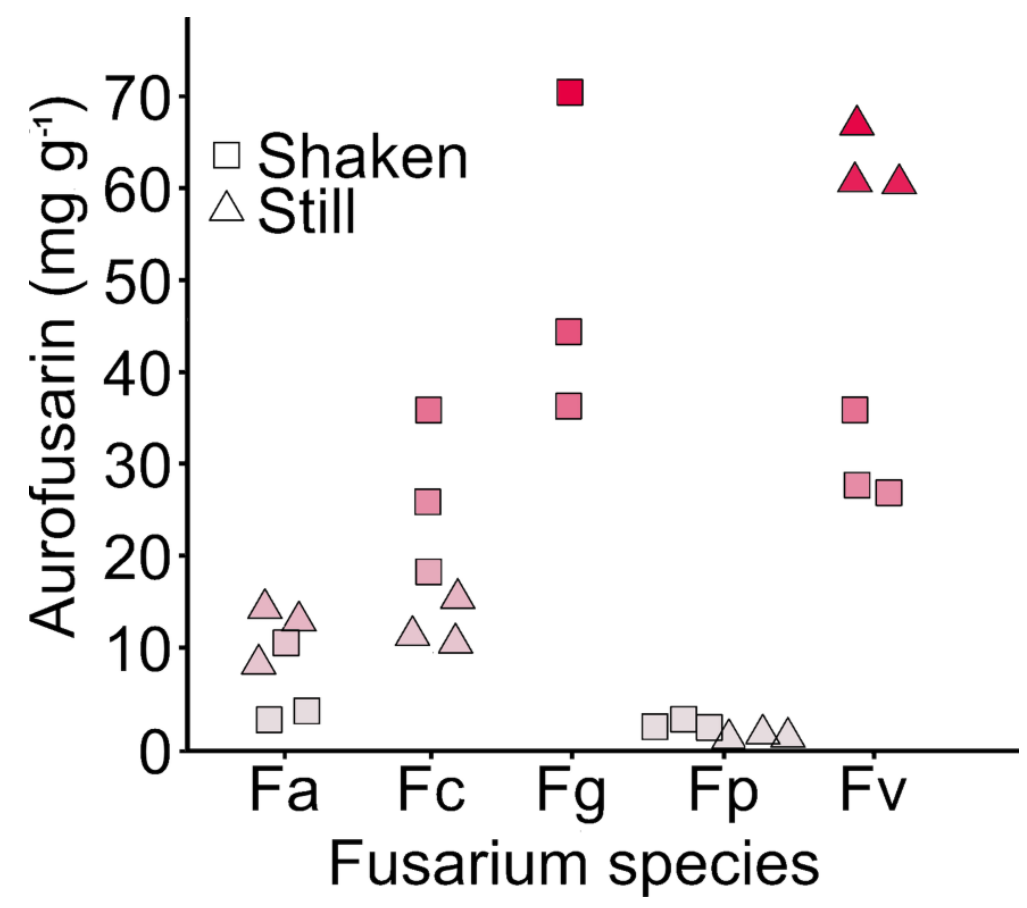

Supplementary Fig. 3 The concentration of aurofusarin in mycelia of Fusarium avenaceum BBA92013 (Fa), F. culmorum $3.37(\mathrm{Fc})$, F. graminearum IFA66 (Fg), F. poae DSMZ62376 (Fp), and $F$. venenatum RD90 (Fv) were grown in still and shaken (140 rpm) cultures in PDB for $14 \mathrm{~d}$ at $23^{\circ} \mathrm{C}$, mycelia were harvested by filtration, freeze-dried, extracted with chloroformmethanol (80:20) and aurofusarin content was determined by HPLC-MS/MS. The coloration of symbols indicates aurofusarin concentration in mycelia. Source data are provided in a Source Data file. 


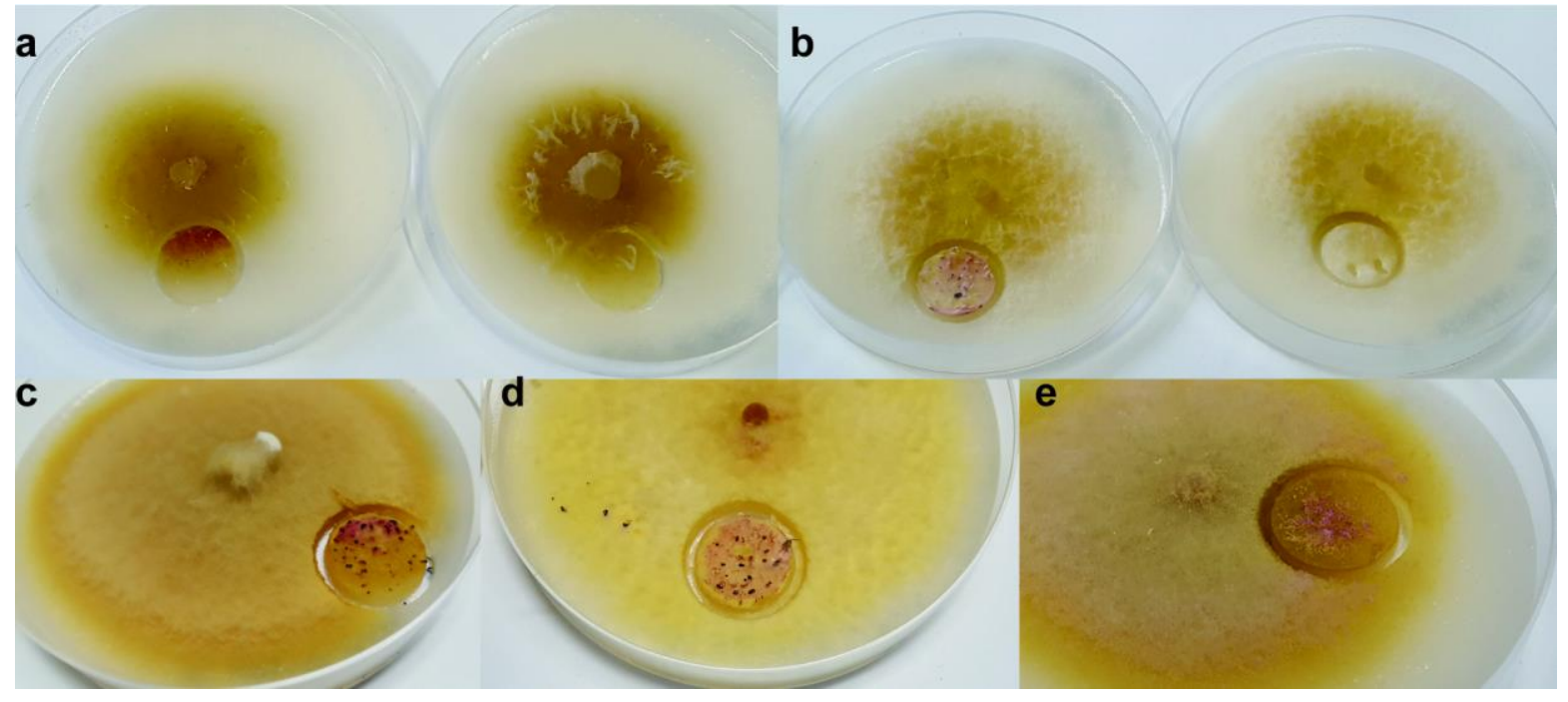

Supplementary Fig. 4 Stimulation of the synthesis of red pigment in Fusarium spp. by grazing. a, Left: $F$. poae DSMZ 62376 culture on rice agar with an arena exposed to $F$. candida grazing for 2 d; right: control with the same arena without animals. b, F. venenatum RD15 culture with an arena exposure to $F$. candida grazing for $2 \mathrm{~d}$; right: control with the same arena without animals. c, F. sporotrichoides IPP 0249 culture with an arena exposed to the isopod Porcellio scaber for 2 d. d, F. venenatum RD 90 culture with an arena exposure to the isopod Porcellio scaber for 2 d. e, F. avenaceum BBA92013 culture with an arena exposure to $F$. candida for $2 \mathrm{~d}$. The animals were confined to grazing arenas of $15 \mathrm{~mm}$ diameter with plastic cylinders inserted into the agar; the cylinders and animals were removed before the photos were taken. 


\section{Fungal strains}

$\longrightarrow$ F. verticillioides

$\longrightarrow$ - F. graminearum

$\longrightarrow$ F. graminearum $\triangle \mathrm{DON}$
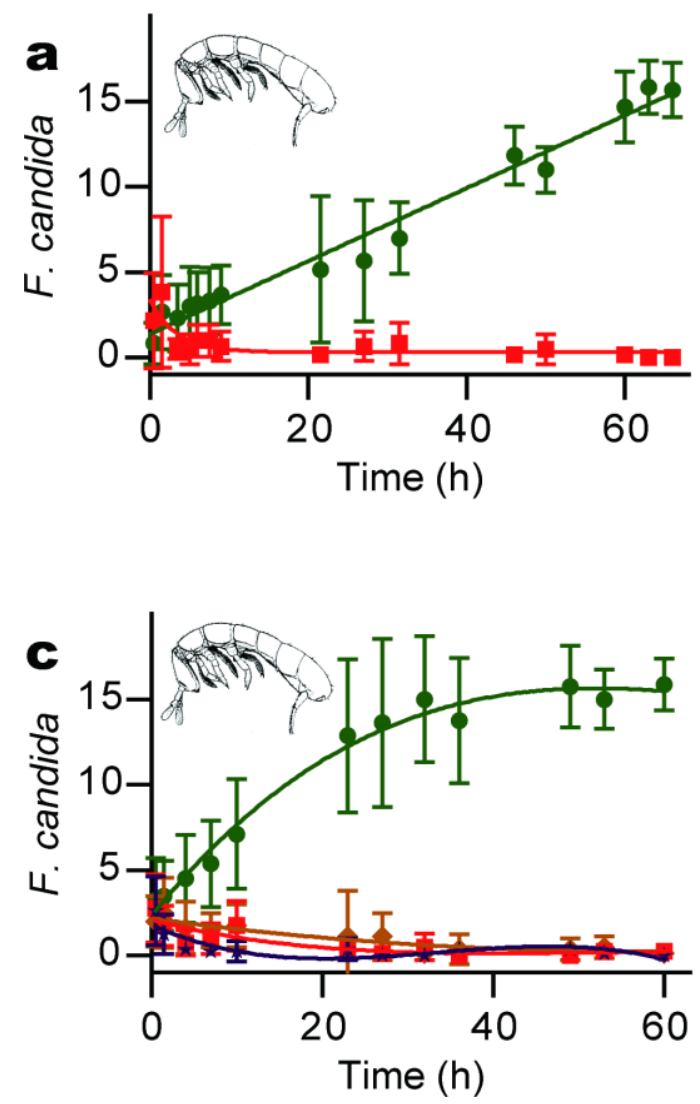
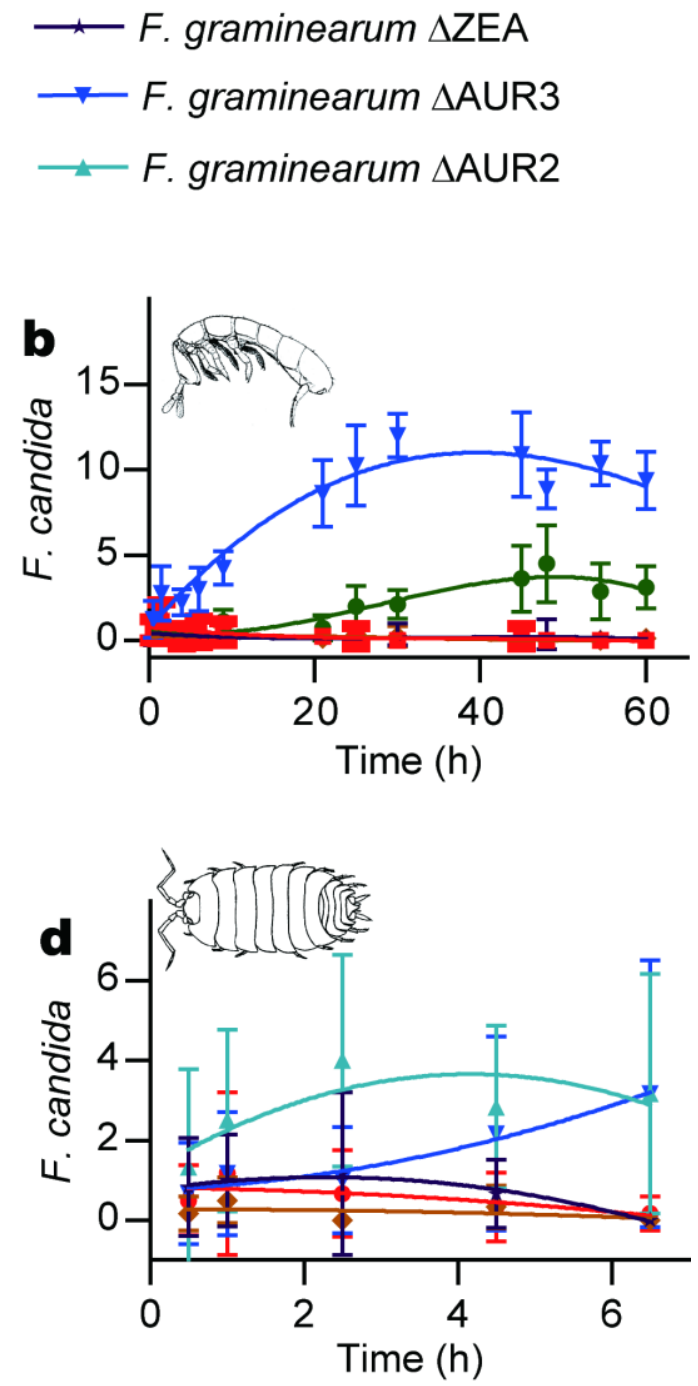

Supplementary Fig. 5 Effect of aurofusarin and mycotoxins deoxynivalenol and zearalenone on the food preference in arthropods. a Preference of the springtail $F$. candida for Fusarium verticillioides over F. graminearum. The springtails starved for two days were placed into the centre of a Petri dish with the fungal cultures on the opposite sides of the dish and the number of animals feeding on each culture was monitored (20 animals per plate; 6 replicates). b Disruption of aurofusarin synthesis in $F$. graminearum ( $\triangle \mathrm{AUR} 2, \triangle \mathrm{AUR} 3)$ reversed the preference of F. candida for Fusarium species; disruption of zearalenone ( $\triangle \mathrm{ZEN})$ and deoxynivalenol $(\Delta \mathrm{DON})$ synthesis did not affect the food preference. The fungal cultures were placed along inner edges of 
Petri dishes in equal distances (20 animals per plate; 8 replicates). c $F$. candida preferred $F$. verticillioides over $F$. graminearum wild type and $F$. graminearum strains with disrupted synthesis of zearalenone and deoxynivalenol (20 animals per plate; 8 replicates). d Isopod T. tomentosa preferred $F$. graminearum strains with disrupted synthesis of aurofusarin over wild type strain and strains with disrupted synthesis of zearalenone and deoxynivalenol ( 8 animals per plate; 6 replicates). Error bars show 95\% CI. Source data are provided in a Source Data file.

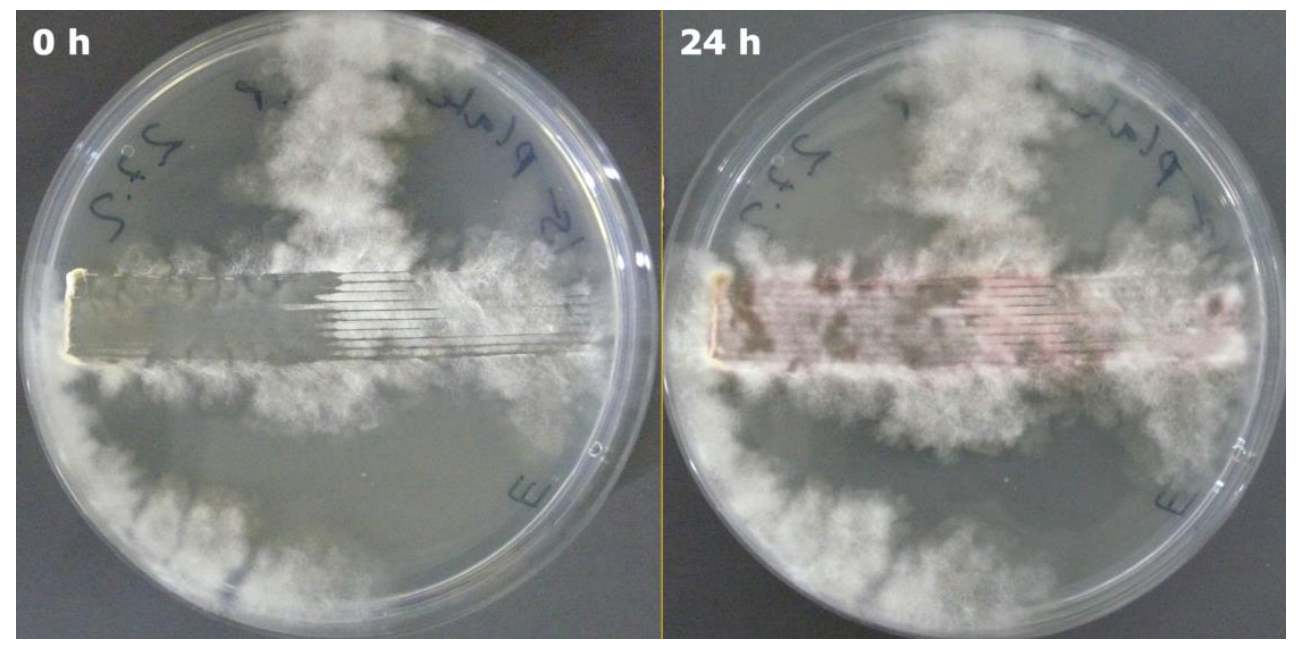

Supplementary Fig. 6 Effect of mechanical damage on Fusarium venenatum. Culture of F. venenatum RD15 growing on GM7 medium at was injured with a razor blade array as in Fig. 6 and photos were taken immediately after the injury (left) and $24 \mathrm{~h}$ later (right). 


\section{Article IV: Small-Scale Bioreactor for Sterile Hydroponics and Hairy Roots: Metabolic Diversity and Salicylic Acid Exudation by Hairy Roots of Hyoscyamus niger}

Zana Jamal Kareem ${ }^{1,2} \dagger$, Ling Su ${ }^{1 \dagger}$, Anna Rathgeb ${ }^{1}$, Anke Sirrenberg ${ }^{1}$, Franz Hadacek ${ }^{1}$, Ahmad Hama Ameen H. Rashid ${ }^{2}$ and Petr Karlovsky ${ }^{1, *}$

$\dagger$ These authors contributed equally to this work.

The article was published online in Applied Sciences 9 (15), p. 3044 in July 2019. The full article can be found online at:

https://www.mdpi.com/2076-3417/9/15/3044

DOI: $\underline{10.3390 / a p p 9153044}$

\section{Author contribution:}

Ling Su carried the sequence analysis, including primer design, PCR, sequencing and data processing. She was also involved in processing metabolic analysis data, visualization and manuscript writing. 
Article

\title{
Small-Scale Bioreactor for Sterile Hydroponics and Hairy Roots: Metabolic Diversity and Salicylic Acid Exudation by Hairy Roots of Hyoscyamus niger
}

\author{
Zana Jamal Kareem ${ }^{1,2, \dagger}$, Ling Su ${ }^{1, \dagger}$, Anna Rathgeb ${ }^{1}$, Anke Sirrenberg ${ }^{1}$, Franz Hadacek ${ }^{1}$, \\ Ahmad Hama Ameen H. Rashid ${ }^{2}$ and Petr Karlovsky ${ }^{1, * \mathbb{D}}$ \\ 1 Molecular Phytopathology and Mycotoxin Research, University of Goettingen, 37077 Goettingen, Germany \\ 2 College of Agricultural Sciences, University of Sulaimani, 334 Sulaimani, Iraq \\ * Correspondence: pkarlov@gwdg.de; Tel.: +49-(0)551-39-12918 \\ + These authors contributed equally to this work.
}

Received: 1 July 2019; Accepted: 25 July 2019; Published: 28 July 2019

check for updates

\begin{abstract}
The volume and complexity of commercial bioreactors for sterile hydroponics and hairy roots are too large for comparative analysis of many cultures. Here a small-scale bioreactor fabricated from standard glass materials and suitable for both airlift and bubble aeration mode is described. The performance of the bioreactor was tested by growing oilseed rape (Brassica napus L.) and rose plants (Rosa canina L.) in sterile hydroponics and by cultivating hairy roots of henbane (Hyoscyamus niger L.) and sesame (Hyoscyamus niger L.). Plants grown in hydroponics for up to six weeks did not show chloroses or necroses. Hairy roots grew faster or comparably fast in bioreactors as compared to shaking flasks. Root exudates of roses and exudates of hairy roots of henbane were subjected to targeted and nontargeted analysis by HPLC coupled with optical and mass spectrometric detectors. The diversity and concentration of hairy root exudates were higher in bioreactors than in shaking flasks. The composition of hairy root exudates of three accessions of $H$. niger did not match the genetic relatedness among the accessions. Hairy roots of Hyoscyamus niger exuded salicylic acid in amounts varying among plant accessions and between bioreactors and shaking flask cultures.
\end{abstract}

Keywords: hairy roots; root exudates; bioreactor; salicylic acid; Hyoscyamus niger; metabolic diversity

\section{Introduction}

Roots of land plants exude small molecules, oligosaccharides, and proteins into the soil. The secreted compounds called root exudates serve multiple functions: they facilitate mobilization of soil nutrients [1-3], modulate the composition of microbial communities of the rhizosphere [4], and attract or repel soil micro- and mesofauna, affecting crop health and productivity [5]. Exudation of metabolites by roots is modulated by nutrient availability [6] and herbivore infestation [7]. Root exudates in turn serve as chemical signals for symbiotic and pathogenic microorganisms [8-10], soil herbivores and their predators [11].

Hairy roots are plant roots genetically transformed with the help of Agrobacterium rhizogenes that exhibit neoplastic growth and can be maintained indefinitely in sterile media without externally added phytohormones [12,13]. Because hairy root cultures consist entirely of differentiated roots and can be easily cloned, they are an ideal model for studies of root biochemistry [14,15] and root-specific processes such as nodulation [16] and the synthesis of root-specific metabolites [17]. Hairy roots also proved useful in studies of interactions between crop plants and herbivores [18]. Biomass production in hairy root cultures is higher than in callus or suspension cultures, therefore hairy roots have been exploited for the production of plant secondary metabolites [12,19-22]. This strategy appears 
particularly promising for large-scale production of metabolites that are primarily synthesized in the roots, such as certain tropane alkaloids of medical interest [23] and glucosinolates with chemoprotective potential [24]. Optimization of the activity of desired biosynthetic pathways can further increase the efficiency of secondary metabolite production by hairy roots [25-28]. Recently genetic engineering of hairy roots received a boost by an adaptation of CRISPR/Cas9 technology for delivery by A. rhizogenes, which combines genome editing and hairy root induction into a single step [29]. Root exudates of hydroponically grown genetically engineered plants [30] as well as hairy roots of genetically modified plants [31,32] can also be used for the production of proteins for diagnostic and medical purposes such as antibodies, antigens for immunization, and growth factors. Optimization of the production system requires comparing multiple genetically enhanced lines because the efficiency of promoters used to drive heterologous gene expression varies among transformation events and even among different parts of the same plant [33]. Similarly, the expression of target genes and the yield of the desired products varies among hairy root lines $[21,28]$. Production of natural products by plants that have not been genetically engineered requires testing multiple accessions, too, because secondary metabolite synthesis varies among plant accessions in a way that is often not predictable based on the genotype [34,35]. Furthermore, cultivation conditions strongly affect production of plant secondary metabolites in hydroponics [36] as well as in hairy root cultures [21,37]. For these reasons, multiple cultures have to be grown in parallel during the optimization of hairy root-based production systems.

A major challenge for hairy root cultures is the supply of oxygen [38]. Different concepts have been pursued to improve oxygen supply to hairy roots in mid- and large-scale bioreactors [22,39]. Apart from conventional stirred bioreactors [40] and stirred bioreactors modified to reduce sharing damage [22,41], hairy root cultures have been grown in plastic sleeve reactors [42], bubble reactors [43], mist reactors [44,45], airlift reactors [46], and liquid-dispersed reactors [47]. The implementation of these concepts focused on midscale bioreactors for research on hairy roots and on industrial bioreactors. Micro and nano bubble generators have recently been introduced into bioreactor design [48], inspired by technologies for the delivery of oxygen and ozone in wastewater treatment [49]. The large surface of micro/nano bubbles as compared to conventional bubbles improves oxygen transfer efficiency. The drawback of micro and nano bubble generators is that they require high-pressure pumps. Furthermore, in airlift mode the liquid velocity decreases with bubble radius for bubbles smaller than $100 \mu \mathrm{m}$ [50]. Because micro bubbles are smaller than $50 \mu \mathrm{m}$ and nano bubbles are smaller than $200 \mathrm{~nm}$ [49], micro bubbles and especially nano bubbles are less suitable to drive liquid transfer in airlift bioreactors.

Small-scale inexpensive bioreactors for screening purposes and comparative studies are lacking, limiting our ability to address differences in the exudation potential of hairy root cultures derived from different plant accessions. Studies of the relationship between genetic polymorphism and secondary metabolite accumulation in plant tissues e.g., [34,51,52] and in root exudates [53] calls for comparative analysis of numerous plant accessions. Exclusion of the effect of bacteria and fungi colonizing plants as endophytes, pathogens, and inhabitants of the phyllosphere require axenic conditions, which can most conveniently be achieved in sterile hydroponic cultures. Lack of small and economical incubators for sterile hydroponics limits these studies.

Our laboratory studies the chemical diversity in sesame [34], extending the focus from seeds to roots and hairy roots and their exudates. In another project we study biological functions of salicylic acid, which may fulfill different roles in different species and even in different tissues of the same plant species. Salicylic acid is known to act as a signal of plant defense response in plant shoots [54] but we also found it in xylem sap of plants infected with a fungal pathogen [55] and in root exudates of healthy plants, where it facilitated mobilization of soil phosphorus [1]. Salicylic acid was also reported to participate on shaping microbial communities of the rhizosphere [56], serving as a signal in interorganismal interactions. Comparative analysis of salicylic acid exudation by different plant accessions requires growing multiple sterile cultures in parallel. In another project in our laboratory we study replant disease of roses. This project relies on the analysis of root exudates because we pursue a 
hypothesis explaining the disease by the effects of certain components of root exudates accumulating in the rhizosphere of roses [57]. Root exudates of rose accessions known to suffer from replant disease have to be collected under sterile conditions, their components identified and the phytotoxicity of these components determined. Progress in all three projects has been hampered by the lack of small bioreactors for sterile hydroponics and hairy root cultures.

The objective of this work was to develop a small-volume, low-cost bioreactor and validate its suitability for hydroponic cultures of Brassica napus and Rosa canina, for growing hairy roots of Hyoscyamus niger and Sesamum indicum and for the collection of root exudates and hairy root exudates suitable for HPLC analysis.

\section{Materials and Methods}

\subsection{Bioreactor Design}

The concept and operating principle of the bioreactor are shown on Figure 1. The body of the bioreactor is connected to an external draft tube. In airlift aeration mode, air bubbles released from a glass frit at the bottom of the draft tube saturate medium with oxygen and drive nutrient medium flow between the draft tube and the reactor body (Figure 1A). The aeration mode can be switched from airlift to bubble mode by attaching air supply to the port on the top of the draft tube and closing the port at the bottom of the draft tube (Figure 1A). The facility to control the aeration mode by connecting air supply to an appropriate port of the draft tube with remaining parts of the reactor unchanged is a distinctive feature of the design.

A

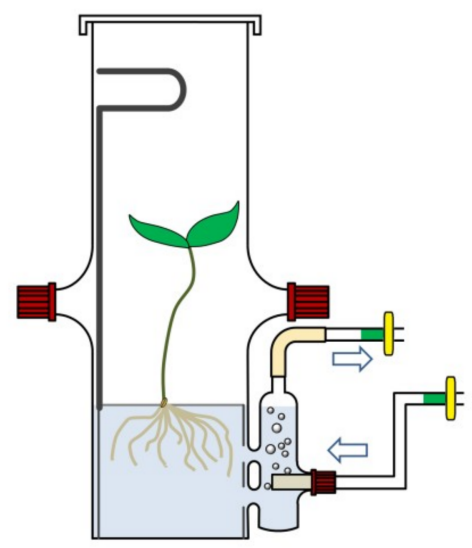

B

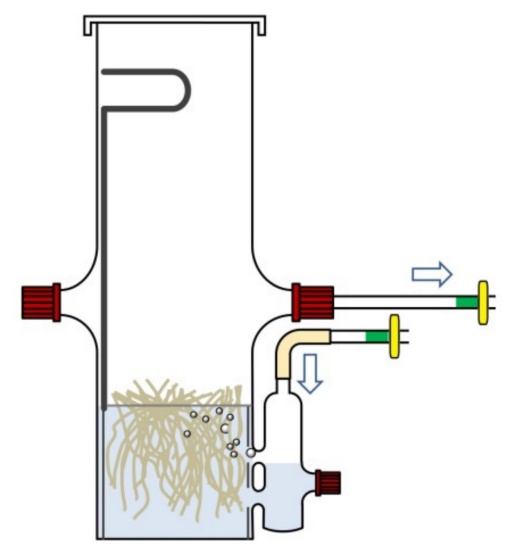

Figure 1. Scheme of bioreactor operating in airlift mode (A) and bubble aeration mode (B).

The second part of the reactor is an internal cylinder inserted into the reactor body. The internal cylinder holds a supporting disk that carries plants or hairy roots. The disk, which loosely resides on the perimeter of the cylinder (Figure 2A, part 1), can be cut from a perforated plate or mesh made of plastic, stainless steel or other materials. The internal cylinder is equipped with a handle for easy removal to facilitate harvesting cultivated material under a sterile bench.

All components and working principles of the bioreactor have been known but the double function of the external draft tube and the way how the bioreactor is assembled from common glass components (see also the following section) are new to this design. 
A

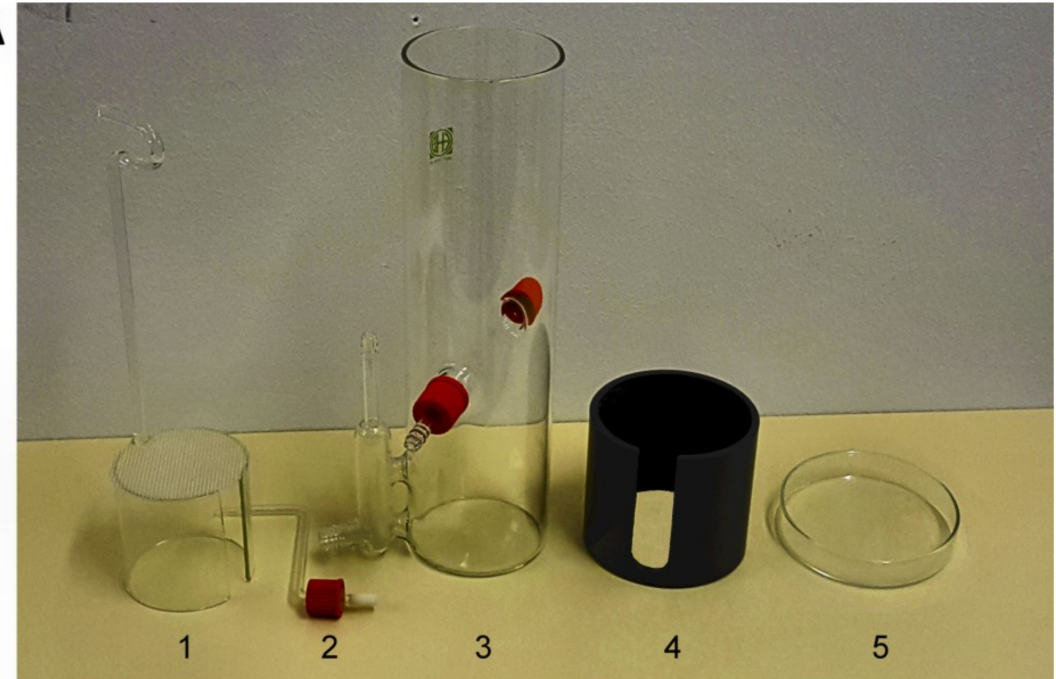

B

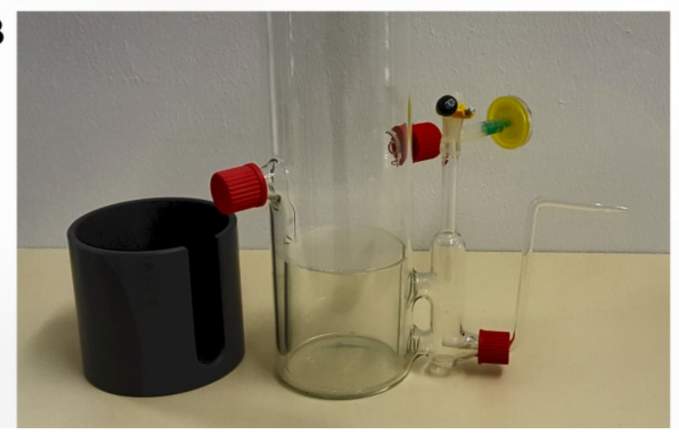

C

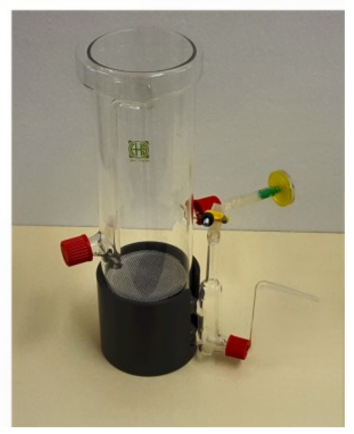

Figure 2. Bioreactor for sterile hydroponics and hairy root cultures. (A) Components of the bioreactor are (1) internal cylinder with a polypropylene mesh, (2) connector for air supply with a glass frit, (3) reactor body with an attached draft tube, (4) light-shielding external cylinder made of polyvinyl chloride, and (5) Petri dish serving as a lid. (B) Detailed view of a bioreactor fitted with an air supply tube. (C) Full view of an assembled bioreactor.

\subsection{Fabrication of the Bioreactor}

The components of the bioreactor are shown in Figure 2A. The reactor body with an attached draft tube (Figure 1A, part 3) and an internal glass cylinder (Figure 1A, part 1) were made of glass tubes. The light-shielding external cylinder (Figure 1A, part 4) was cut from a polyvinyl chloride tube. The body of the reactor had an external diameter of $90 \mathrm{~mm}$, a height of $270 \mathrm{~mm}$ and a wall thickness of $2.5 \mathrm{~mm}$. The draft tube of $18 \mathrm{~mm}$ diameter and $75 \mathrm{~mm}$ height was connected with the reactor body at two sites via glass tubes as shown in Figure 2A, part 3 and Figure 2B. It was furnished with two connectors: a bottom connector accommodated a glass tube with a frit supplying air in airlift operation mode (Figure 2A, part 2). The top connector served as air supply in bubble aeration mode. The body of the reactor was equipped with two ports for the collection of volatiles, for an optional $\mathrm{CO}_{2}$ supply or for air exhaust in bubble aeration mode. Ports that were not in use were closed with screw cups. The internal cylinder had a diameter of $84 \mathrm{~mm}$ and a height of $70 \mathrm{~mm}$. The cylinder held a polypropylene mesh, which rested on its perimeter as a support for plants or hairy roots during cultivation (Figure 2A, part 1). A handle attached to the internal cylinder was made of a glass rod of a diameter of $5 \mathrm{~mm}$. A clearance of $20 \mathrm{~mm}$ width was cut into the internal cylinder (Figure 2A, part 1) at a position facing the draft tube (Figure $1 \mathrm{~A}, \mathrm{~B}$ ) to allow exchange of growth medium between the body of the reactor and the draft tube and to facilitate air supply in bubble aeration mode. A glass Petri dish with an internal diameter of 91-92 mm served as a lid (Figure 2A, part 5). 


\subsection{Operation of the Bioreactor}

For sterile hydroponics, the reactor was filled with nutrient solution just to the level of the mesh supporting the plants, which required about $450 \mathrm{~mL}$ medium per reactor. The reactor was operated in airlift mode with the upper port of the draft tube closed with a filter and the air supply attached to the lower port of the draft tube. The air flow was $480 \mathrm{~mL} / \mathrm{min}$.

For growing hairy roots, the reactor was steam-sterilized and filled with nutrient solution to the level of the supporting mesh or above, depending on whether aerial growth was desirable. Hairy root cultures are known to suffer from inhomogeneous growth and inferior yields when grown in air-lift reactors [46], therefore bubble aeration mode was used. The air flow was $670 \mathrm{~mL} / \mathrm{min}$. In some experiments, the lid was loosely place over the reactor, both ports of the body of the reactor were closed with screw cups to and the flow of air leaving the reactor below the led prevented contamination. In other experiments, the lids were sealed in place with Parafilm and one of the ports of the reactor body serving as an air exhaust was equipped with a hydrophobic sterile filter (Midisart, Sartorius, Göttingen, Germany).

Low-cost aquarium pumps connected to the reactors via Midisart filters were used as air supply. The reactors were operated in an environmental chamber (Mytron, Bio-u. Solartechnik GmbH, Heiligenstadt, Germany) set to a constant temperature (varying among the experiments as described in Section 3) and $12 \mathrm{~h}$ light per day. We have not measured oxygen concentration in growth medium because the concentration within in the bulk of hairy roots is likely to vary with the location of the probe and accurate methods for the determination of volumetric mass transfer coefficient, such as a rapid switch between input gases with different oxygen concentrations or the dynamic pressure method [58], were beyond the scope of this work.

\subsection{Plant Material}

Seeds of Brassica napus var. napus, rapid cycling accession were provided by P.H. Williams (Crucifer Genetics Cooperative at the University of Wisconsin-Madison, WI, USA). Sterile cuttings of rose plants (Rosa canina L., variety "The Fairy") were purchased from Institut für Pflanzenkultur (Schnega, Germany). Accessions of Hyoscymus niger and Sesamum indicum used in this study are listed in Tables 1 and 2.

Table 1. Hyoscyamus niger.

\begin{tabular}{cccc}
\hline Label & Origin & Year & Source \\
\hline Iraq & Iraq, Daray Mar & 2014 & Collected by ZJK \\
Iran & Iran, Isfahan & 2015 & Pakan Bazr * \\
Germany & Germany, Göttingen & 2016 & Botanical garden \\
\hline \multicolumn{4}{c}{ * Seeds were provided by Pakan Bazr company, Iran. }
\end{tabular}

Table 2. Sesamum indicum accessions.

\begin{tabular}{cccc}
\hline Label & Origin & Year $^{*}$ & Accession \\
\hline India & India, Hyderabad & 2004 & Acc. 92-2918 \\
Japan & Japan & 2004 & Acc. 92-3030 \\
Sumer S2b & Iraq, Erbil & 2014 & Sumer S2b \\
\hline
\end{tabular}

* Year of the acquisition of seeds from the collection. ${ }^{* *}$ Centro Nacional Inv. Agropecuarias (CENIAP) Germplasm Bank, Venezuela. ${ }^{* *}$ Ministry of Agriculture, Regional Government of Iraqi Kurdistan, General Directorate of Research, Extension and Training.

\subsection{Analysis of Root Exudates by HPLC-DAD}

Growth medium was filtered through paper filter to remove root fragments and concentrated in a rotary evaporator to $1 / 4$ volume. The concentrate was extracted with equal volume of ethyl acetate, the watery phase was acidified with $1 \%$ acetic acid and extracted with a new portion of ethyl acetate. 
The extracts were combined and solvent was removed in vacuum. Three biological replicates were prepared and extracts of culture medium incubated in bioreactors under the same conditions yet without plants were used as blanks. Thirty-three milligrams of dried residue were suspended in $1.5 \mathrm{~mL}$ of methanol/water (1:1) and sonicated for $5 \mathrm{~min}$. The suspension was filtered through $0.4 \mu \mathrm{m}$ filters and $10 \mu \mathrm{l}$ were injected into HPLC system 1290 Infinity II with a DAD detector G7117B (Agilent, Darmstadt, Germany) equipped with a Varian Polaris C18 Ether column ( $3 \mu \mathrm{m}$ particle size, $2 \times 100 \mathrm{~mm}) \mathrm{kept}$ at $35{ }^{\circ} \mathrm{C}$. LC-MS grade methanol and distilled water were acidified with $0.1 \%$ formic acid and used for gradient elution from $95 \%$ to $0 \%$ water in $20 \mathrm{~min}$ at a flow rate of $0.2 \mathrm{~mL} / \mathrm{min}$. Light absorption spectra were recorded from 220 to $550 \mathrm{~nm}$ and chromatograms at the wavelengths 229, 254, 280, and $320 \mathrm{~nm}$ were monitored. The bandwidth was set to $4 \mathrm{~nm}$ and the reference wavelength to $600 \mathrm{~nm}$.

\subsection{Induction of Hairy Roots}

Agrobacterium rhizogenes ATCC 15834 was grown in YEB medium [59] (0.5\% peptone, $0.1 \%$ yeast extract, $0.5 \%$ meat extract, $0.5 \%$ sucrose, $0.049 \% \mathrm{MgSO}_{4} .7 \mathrm{H}_{2} \mathrm{O}, 1.5 \%$ agar, $\left.\mathrm{pH} 6.8-7.2\right)$, at $25-26{ }^{\circ} \mathrm{C}$ for $36 \mathrm{~h}$ in the dark on a rotatory shaker $(100 \mathrm{rpm})$. The cells were harvested by centrifugation at $5000 \times g$ for $15 \mathrm{~min}$, re-suspended in MS medium (Murashige and Skoog medium, [60]) and set to the $\mathrm{OD}_{600}$ of 1.3 . Leaves were harvested from four weeks old plants grown under sterile conditions from superficially sterilized seeds, cut into squares of $1 \times 1 \mathrm{~cm}$, placed on $1 / 2 \mathrm{MS}$ medium solidified with $0.7 \%$ agar and co-cultivated with $A$. rhizogenes for $30 \mathrm{~min}$ at room temperature. After the incubation, leaf segments were dried with sterile filter paper and placed onto phytohormone-free MS medium supplemented with $2.2 \%(\mathrm{w} / \mathrm{v})$ sucrose and solidified with $0.7 \%$ agar and incubated in darkness at $22{ }^{\circ} \mathrm{C}$. After $72 \mathrm{~h}$ the segments were transferred onto fresh medium supplemented with cefotaxime at $500 \mathrm{mg} / \mathrm{L}$. About two weeks after the inoculation the formation of the first roots was observed. One root was excised from each leaf segment. These roots were treated as hairy root clones. The roots were subcultured each 30 days. After six passages, sterile hairy roots were transferred to antibiotic-free medium.

\subsection{Analysis of Hairy Root Exudates by HPLC-ELSD}

Three biological replicates were prepared and culture medium was used as a blank. The samples were analyzed by HPLC using Agilent 1290 Infinity II system coupled to an Agilent 1260 evaporative light-scattering detector (ELSD) (Agilent, Darmstadt, Germany). For the ELSD, the evaporation temperature was set to $42{ }^{\circ} \mathrm{C}$, the nebulizer temperature to $40{ }^{\circ} \mathrm{C}$ and the nebulizer $\mathrm{N}_{2}$ flow amounted $1.6 \mathrm{~L} / \mathrm{min}$. A Varian Polaris C18 Ether column $(3 \mu \mathrm{m}$ particle size, $2 \times 100 \mathrm{~mm})$ was used for separation. The column temperature was kept at $35^{\circ} \mathrm{C}$ and samples of $40 \mu \mathrm{L}$ were injected. LC-MS grade methanol obtained from Chemsolute (Th. Geyer, Renningen, Germany) and distilled water purified by Arium Pro water purification system (Sartorius, Göttingen, Germany) were acidified with $0.1 \%$ LC-MS grade formic acid (Honeywell Fluka, Bucharest, Romania) and used for gradient elution from $90 \%$ to $2 \%$ water in $15 \mathrm{~min}$ at a flow rate of $0.2 \mathrm{~mL} / \mathrm{min}$.

\subsection{Analysis of Salicylic Acid Content in Hairy Root Exudates by HPLC-MS}

The samples were analyzed by HPLC using an Agilent 1290 Infinity II system (see Section 2.7 for details) with the column maintained at $35^{\circ} \mathrm{C}$. LC-MS grade methanol and distilled water were acidified with $0.1 \%$ formic acid and used for gradient elution from $95 \%$ to $0 \%$ water in $20 \mathrm{~min}$ at a flow rate of $0.2 \mathrm{~mL} / \mathrm{min}$. The HPLC system was coupled to an Agilent 6545 QTOF equipped with a Jet Stream source operated with the following settings: drying gas temperature $320^{\circ} \mathrm{C}$, drying gas flow $8 \mathrm{~L} / \mathrm{min}$, nebulizer pressure $35 \mathrm{psig}$, sheath gas temperature $350{ }^{\circ} \mathrm{C}$, and sheath gas flow $11 \mathrm{~L} / \mathrm{min}$. Negative mode data were acquired with three spectra pre second recorded from 100 to $1700 \mathrm{~m} / \mathrm{z}$. External calibration was prepared from salicylic acid solutions in 50\% methanol. 


\subsection{Primer Design and Sequence Analysis}

The sequence of $\operatorname{Tr} 2$ gene from Hyoscyamus niger (accession No. AB026545.1, tropinone reductase II) was used for the design of primers for PCR and sequencing. The primers (forward primer TCTGA ACGAG AAGAG TTTAT GAAGA and reverse primer AAGTG ATAAG CAGCC TCAAA GT) amplified a segment of the gene from $1543 \mathrm{nt}$ to $2237 \mathrm{nt}$, producing an amplicon of $695 \mathrm{bp}$. PCR products were sequenced by Sanger method from both ends (Macrogen Europe, Amsterdam, The Netherlands), the complementary reads were aligned and ambiguities were resolved by inspection of the electropherograms. An alignment of the sequences from $1592 \mathrm{nt}$ to $2165 \mathrm{nt}$ (original numbering of AB026545.1) was constructed using Clustal W implemented in MEGA 10.0.4 [61] and used to calculate nucleotide differences per $1000 \mathrm{bp}$.

\section{Application Examples}

The performance of the bioreactor was tested by growing two plant species in sterile hydroponics and collecting root exudates; by comparing growths rate of hairy roots generated from two plant species; and by collecting and analyzing hairy root exudates.

\subsection{Growing Oilseed Rape (Brassica napus L.) in Sterile Hydroponics}

Ten surface-sterilized seeds of a rapid cycling variety of Brassica napus were placed into bioreactors filled with half-concentrated MS medium [60] without sucrose and operated in airlift mode. The plants grown at $24^{\circ} \mathrm{C}$ for three weeks have not shown any chloroses or other negative effects of cultivation in restricted space and at high air humidity [62,63] (Figure 3).

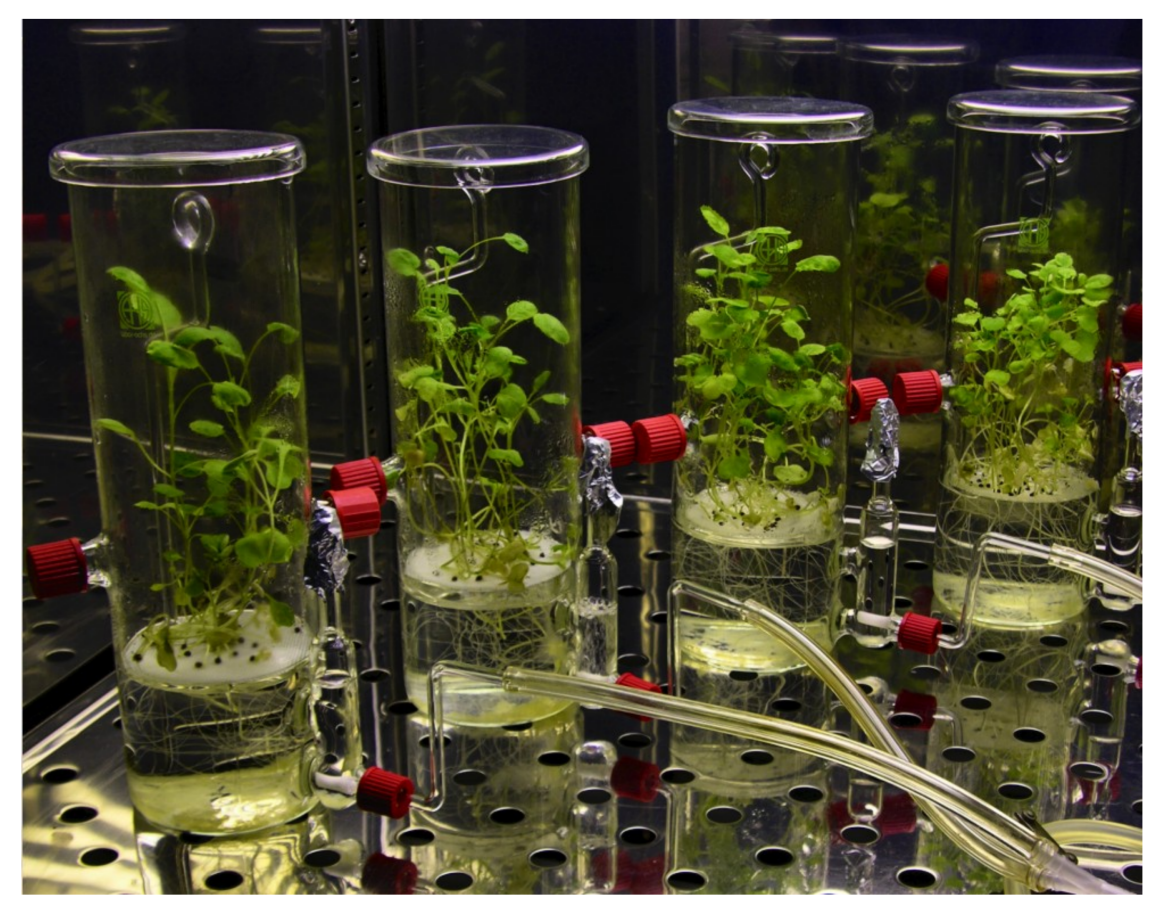

Figure 3. Oilseed rape (Brassica napus L.) grown in sterile hydroponics. Ten plants per bioreactor were grown in $0.5 \times$ MS medium for three weeks at $24{ }^{\circ} \mathrm{C}$ with $12 \mathrm{~h}$ light per day. External light-shielding cylinders were removed for the photography.

\subsection{Growing Rose Plants (Rosa canina L.) in Sterile Hydroponics}

Rose cuttings were adapted to light for 3 days at $20^{\circ} \mathrm{C}$ under long day conditions before transfer to bioreactors. A single rose plant per bioreactor was cultivated for six weeks in half-concentrated MS 
medium with $2 \mathrm{~g}$ sucrose per liter and $\mathrm{pH}$-value set to 5.8 with $\mathrm{KOH}$ in bioreactors in the airlift mode (Figure 4). The plants were grown at $20^{\circ} \mathrm{C}$ for four weeks.

All roots formed on cuttings were adventitious by definition, yet strong white roots penetrating the plastic mesh and growing downwards resembled primary and seminal roots, while dark roots growing on the surface of the mesh and supporting the plants in a vertical position resembled crown roots (Figure 4). The roots growing downwards in medium remained white for the entire cultivation period without signs of necroses, which typically occur in medium solidified with agar (A. Sirrenberg, unpublished observation) due to replant disease syndrome [57].

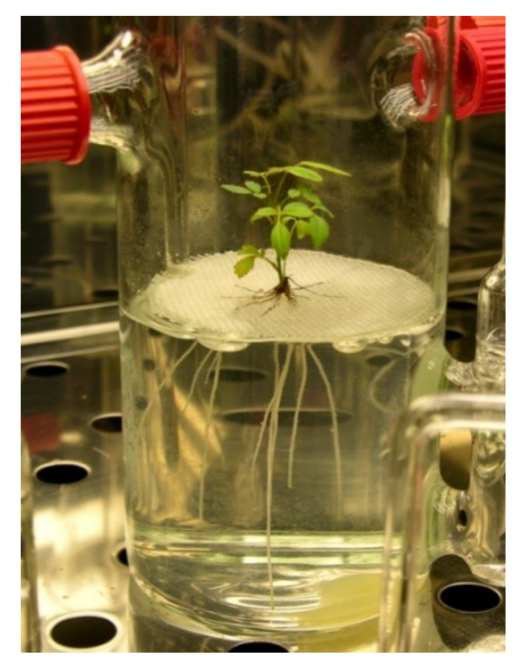

Figure 4. Rose plant (Rosa canina L.) growing in sterile hydroponics in $0.5 \times$ Murashige-Skoog medium supplemented with $2 \%$ sucrose in a bioreactor operated in airlift mode.

\subsection{Collection and Analysis of Root Exudates of Rose (Rosa canina L.) by HPLC-DAD}

Rose plants (Rosa canina L.) were grown in medium with $2 \%$ sucrose for the collection of root exudates as shown in Figure 4. After four weeks, plants were removed and medium was filtered and stored at $-20^{\circ} \mathrm{C}$. For the chemical analysis of root exudates, exclusion of microbial contamination is crucial because microorganisms use the exudates as nutrients and contaminate the medium with their own metabolites. In 11 experiments with 3 to 6 parallel cultures each, contamination occurred in less than $5 \%$ cultures. A total of $13.2 \mathrm{~L}$ medium were collected, concentrated in vacuum and extracted with ethyl acetate (see Section 2.5.) for the analysis and purification of selected components of root exudates. Figure 5 shows a typical result of the analysis of root exudates of rose plants collected in bioreactors by HPLC coupled to a UV detector (HPLC-DAD).

\subsection{Growing Hairy Roots of Hyoscyamus niger and Sesamum indicum}

To compare the yield of hairy roots in the bioreactor and in shaken flask cultures, hairy roots originating from three accession of each Hyoscyamus niger and Sesamum indicum were cultivated in bioreactors and in shaking flasks. The cultures were initiated by $100 \mathrm{mg}$ of hairy roots cut into $1 \mathrm{~cm}$-long pieces. Culture flasks of $300 \mathrm{~mL}$ volume contained $50 \mathrm{~mL}$ MS medium supplemented with $2.5 \%$ sucrose. The cultures were grown on a shaker at $90 \mathrm{rpm}$ in the dark at $25^{\circ} \mathrm{C}$ and in bioreactors operated in bubble mode with an air flow of $670 \mathrm{~mL} / \mathrm{min}$ (Figure 6).

After 30 days, hairy roots were harvested by filtration, blotted dry with sterilized filter paper, frozen at $-20^{\circ} \mathrm{C}$ overnight and lyophilized for $72 \mathrm{~h}$. Then the dry weight of hairy roots was determined (Figure 7). 
A

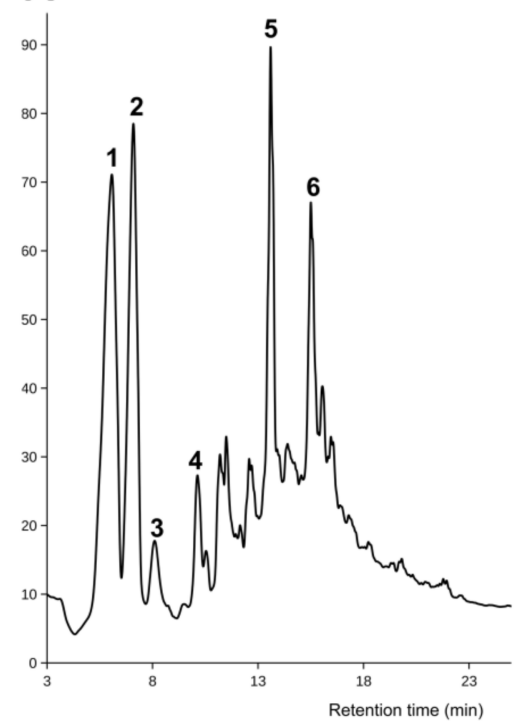

B
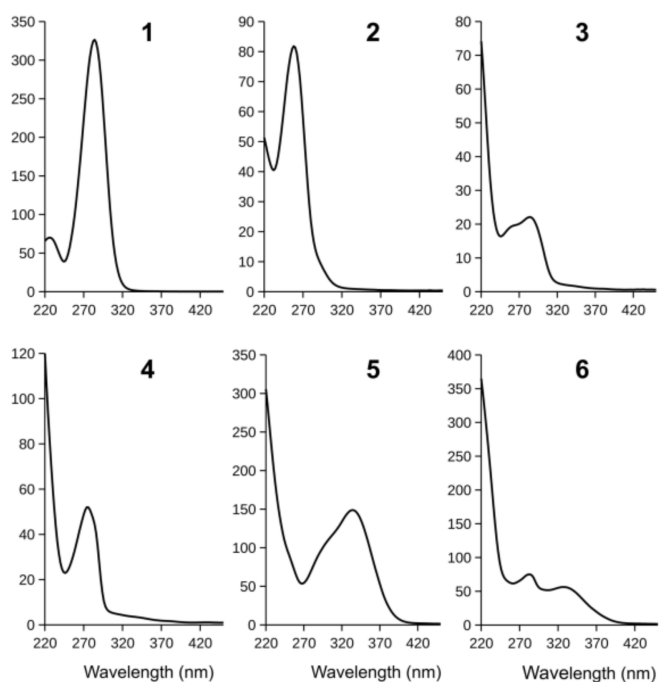

Figure 5. HPLC analysis of root exudates of rose (Rosa canina L.) collected in the bioreactor. (A) HPLC chromatogram of root exudates separated on a reverse-phase column, eluted with a methanol gradient and detected by light absorption at $254 \mathrm{~nm}$. (B) UV absorption spectra of major phenolic compounds recorded using a diode array detector coupled to HPLC.

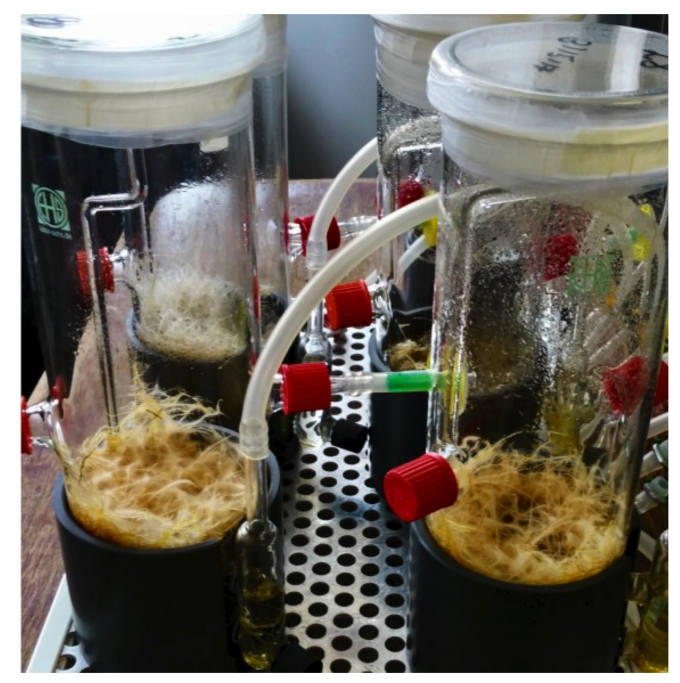

Figure 6. Hairy root cultures generated from several accessions of Hyoscyamus niger in bioreactors operated in a bubble aeration mode.

The biomass of hairy roots varied among accessions but we have not investigated these differences statistically because our purpose was to compare the performance of the bioreactor with that of flask cultures. In Sesamum indicum, the biomass of hairy roots grown in bioreactors was larger than or similar to the biomass harvested from shaking flasks. In Hyoscyamus niger, more biomass was harvested from bioreactors than from flasks in all three plant accessions studied (Figure 7). We assume that better supply of oxygen in the bioreactor was the cause of these differences.

\subsection{Collection and Nontargeted Analysis of Hairy Root Exudates of Sesamum indicum}

Growth medium of hairy root cultures grown as described above was cleared by centrifugation for $10 \mathrm{~min}$ at $2900 \times \mathrm{g}$ to remove root fragments. The medium was used for HPLC-ELSD analysis 
without cleanup. Figure 8 shows a comparison of HPLC-ELSD chromatograms of hairy root exudates obtained from the bioreactor and from a flask culture for one accession of sesame.

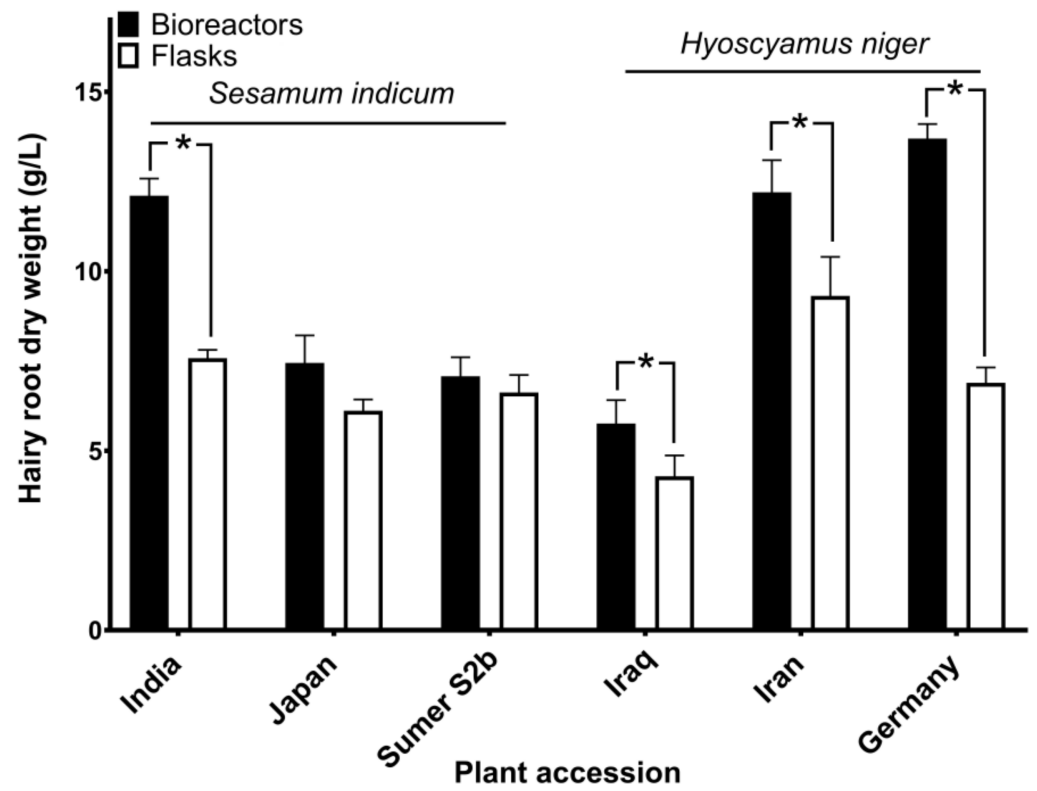

Figure 7. Biomass of hairy roots grown in bioreactors and flask cultures. Hairy root cultures originating from three accessions of each plant species were grown for 30 days and the dry weight of harvested biomass was determined. Asterisks denote significant differences between means (two-tailed $t$-test, $\mathrm{n}=3, \mathrm{p}<0.05)$.

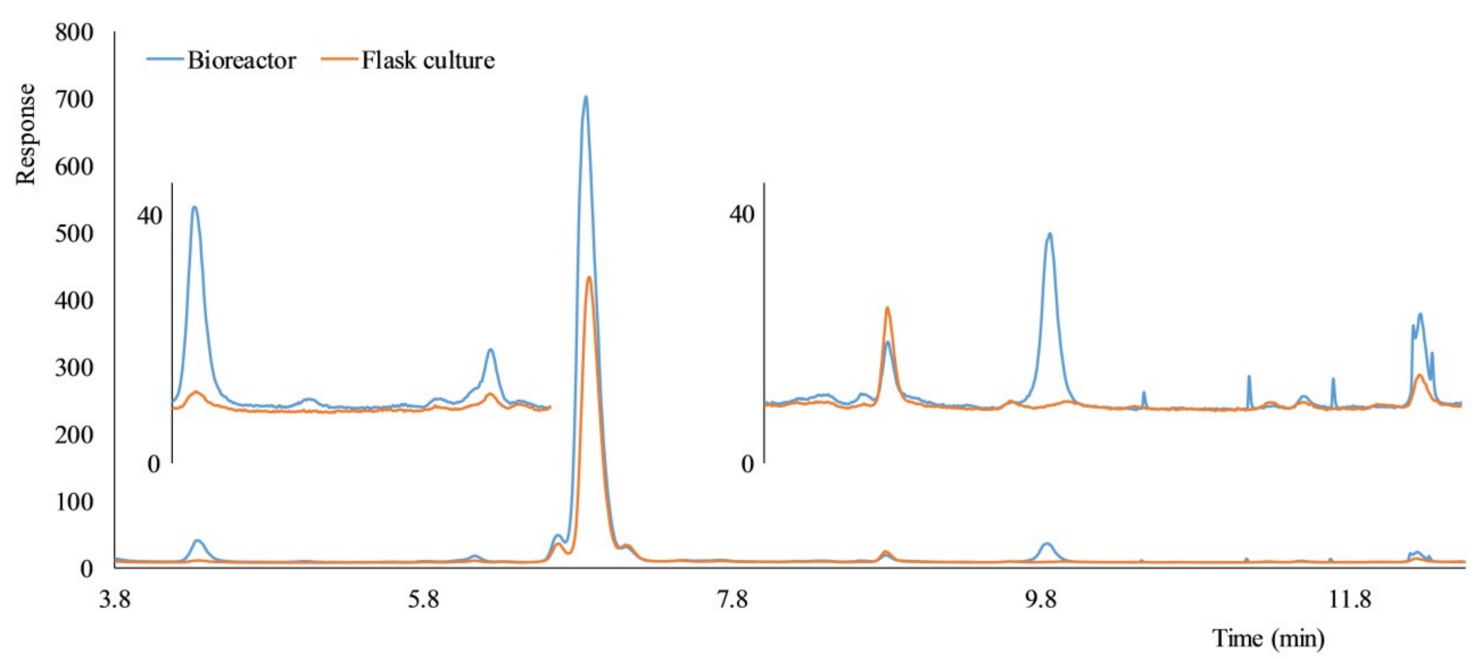

Figure 8. Comparison of HPLC-ELSD chromatograms of hairy roots exudates of Sesamum indicum (accession Japan) grown in the bioreactor and a flask culture. Culture fluid clarified by centrifugation was directly analyzed by HPLC-ELSD.

The amount and diversity of hairy root exudates obtained from a bioreactor were higher than the amount and diversity of exudates obtained from a shaking flask culture (Figure 8). Better oxygen supply in the bioreactors was the likely reason. Most importantly, all metabolic signals recorded in exudates from the flask culture were also found in exudates from the bioreactor, indicating that that few (if any) metabolites will be lost by replacing culture flasks with bioreactors. 


\subsection{Analysis of Salicylic Acid Exudation by Hairy Roots of Hyoscyamus niger}

To test the suitability of the bioreactor for comparative studies of salicylic acid exudation by hairy roots, hairy root cultures generated from three accessions of Hyoscyamus niger and Sesamum indicum were grown in $0.5 \times$ MS medium with $2.5 \%$ sucrose in bioreactors operated in bubble mode and in shaken flasks. After 30 days, growth medium was collected, extracted with ethyl acetate and the extracts were analyzed by HPLC-MS. The results are shown in Figure 9.

Hairy roots of Hyoscyamus niger exuded salicylic acid into growth medium in amounts varying from $2 \mu \mathrm{g} / \mathrm{L}$ to $60 \mu \mathrm{g} / \mathrm{L}$, while hairy root exudates of Sesamum indicum has not secreted detectable amounts of salicylic acid (data not shown). Cultivation conditions strongly affected the concentration of salicylic acid accumulating in nutrient solution. Hairy roots of one plant accession secreted larger amounts of salicylic acid in bioreactors, while hairy roots on another accession secreted much large amounts of salicylic acid in shaking flasks (Figure 9). This surprising result indicates that the response of salicylic acid pathway to environmental cues varies among populations of the same plant species. We speculate that these differences reflect selection pressures exerted by different environments on the plants. The result corroborates our assumption that studying differences in salicylic acid production among plants with different life histories may shed light into biological functions of this multifaceted metabolite.

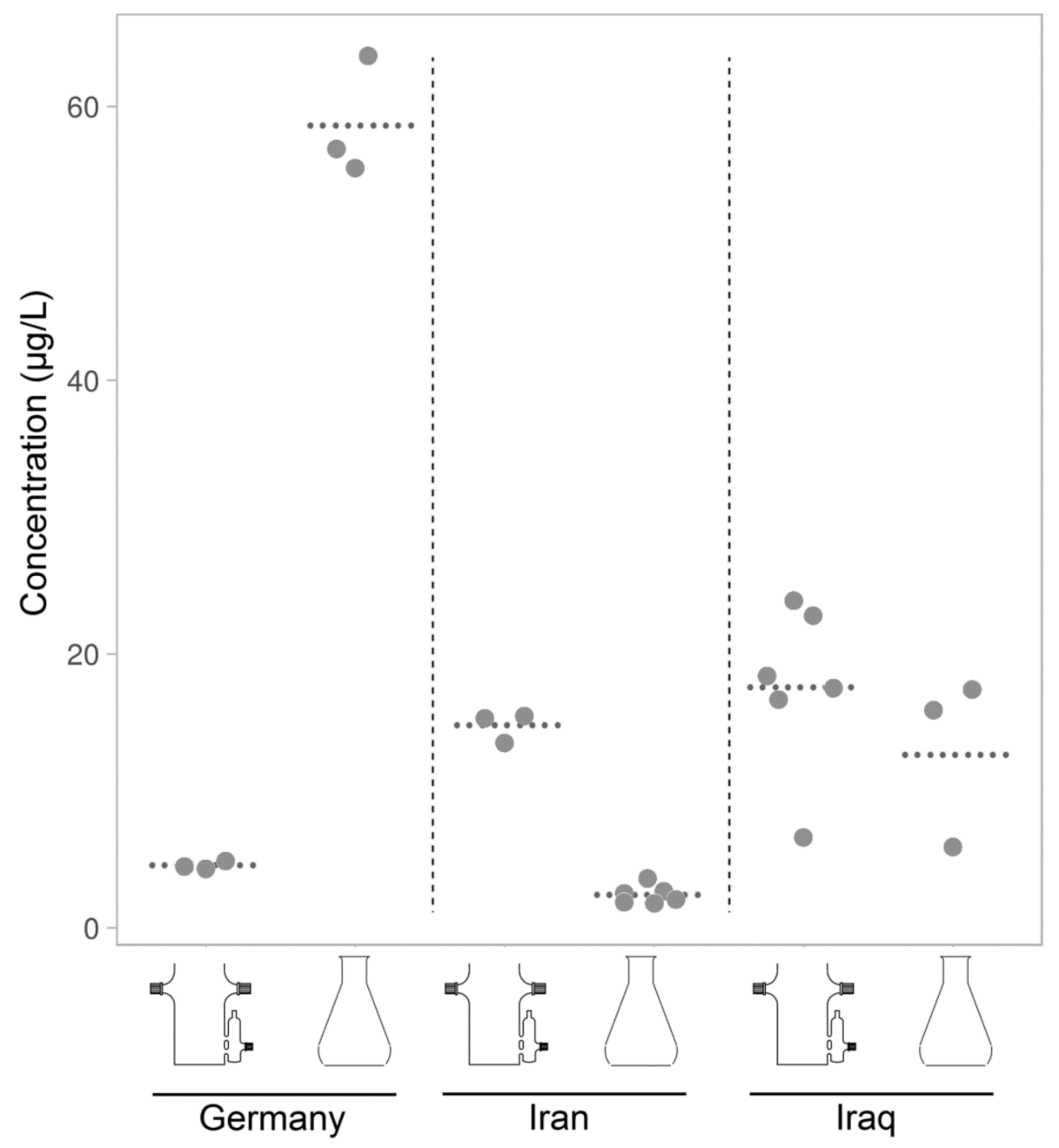

Figure 9. Salicylic acid accumulated in nutrient solution of hairy root cultures derived from three accessions of Hyoscyamus niger (designated Iraq, Iran and Germany) and grown in bioreactors and in shaking flasks for three weeks. Dotted lines show the means. 
As far as we are aware, secretion of salicylic acid by hairy roots has not been reported yet. Adding salicylic acid to culture medium of hairy roots was used to stimulate the synthesis of alkaloids [64], including hyoscyamine and further tropane alkaloids $[65,66]$, which are also produced by Hyoscyamus niger. The interplay between the secretion of salicylic acid and stimulation of alkaloid synthesis by externally added salicylic acid adds to the complexity of biological roles and effects of salicylic acid in plants.

\subsection{Nontargeted Analysis of Hairy Root Exudates in Hyoscyamus niger: Comparison to Shaking Flasks}

Hairy root cultures of $H$. niger were grown in the bioreactor operated in a bubble mode and in traditional shaken flask cultures. After 30 days, growth media were collected, extracted with ethyl acetate and the extracts were analyzed by HPLC. Because the components of the exudates were unknown, an evaporative light scattering detector (ELSD) was used, which allows for an approximate comparison of concentrations without authentic standards because the response factor of the detector is largely independent of the chemical structure of the analyte [67].

The results of the analysis of hairy root exudates by HPLC-ELSD are shown in Table 3 . The average standard error of the intensity of HPLC signals in exudates obtained from bioreactors was $20 \%$, compared to the average standard error of $28 \%$ for exudates from shaken flasks. Thus the reproducibility of the composition of hairy root exudates collected in bioreactors appears higher than the reproducibility of exudates collected in shaking flasks. The total intensity of HPLC-ELSD signals detected in exudates from bioreactors (average total per accession of 9024) was 1.6-times larger than the total intensity of signals from shaking flasks (average total per accession of 5692), indicating that hairy roots exuded larger amounts of metabolites in bioreactors as compared to shaking flasks.

Table 3. Exudates of hairy roots of Hyoscyamus niger collected in bioreactor and flask cultures. The exudates were analyzed by HPLC-ELSD. Only metabolites present in all three replicates were recorded.

\begin{tabular}{lccccccc}
\hline $\begin{array}{c}\text { Accession } \\
\text { Metabolite }\end{array}$ & \multirow{2}{*}{ RT * } & \multicolumn{2}{c}{ Iran } & \multicolumn{2}{c}{ Germany } & \multicolumn{2}{c}{ Iraq } \\
Bioreactor & Flasks & Bioreactor & Flasks & Bioreactor & Flasks \\
\hline Compound 1 & 3.6 & N.d. & $224 \pm 191$ & N.d. & N.d. & N.d. & N.d. \\
Compound 2 & 4.3 & $41 \pm 4$ & N.d. & $41 \pm 4$ & N.d. & N.d. & $100 \pm 125$ \\
Compound 3 & 6.1 & $128 \pm 18$ & $20 \pm 2$ & $24 \pm 1$ & $32 \pm 2$ & $30 \pm 4$ & $41 \pm 2$ \\
Compound 4 & 6.8 & $12,500 \pm 600$ & $2300 \pm 00$ & $5700 \pm 490$ & $5100 \pm 200$ & $7000 \pm 300$ & $5900 \pm 200$ \\
Compound 5 & 7.7 & $29 \pm 5$ & N.d. & N.d. & N.d. & N.d. & N.d. \\
Compound 9 & 9.5 & $40 \pm 8$ & N.d. & N.d. & N.d. & $35 \pm 9$ & N.d. \\
Compound 10 & 9.7 & $75 \pm 29$ & $750 \pm 350$ & $1300 \pm 650$ & $485 \pm 175$ & N.d. & $1750 \pm 490$ \\
Compound 11 & 9.9 & $42 \pm 29$ & N.d. & N.d. & N.d. & N.d. & N.d. \\
Compound 12 & 10.5 & N.d. & N.d. & $32 \pm 2$ & $52 \pm 13$ & N.d. & $120 \pm 30$ \\
Compound 13 & 11.1 & N.d. & N.d. & $51 \pm 18$ & $110 \pm 41$ & N.d. & $67 \pm 2$ \\
Compound 14 & 11.3 & N.d. & N.d. & $21 \pm 5$ & N.d. & N.d. & N.d. \\
Compound 17 & 14.8 & N.d. & $87 \pm 8$ & N.d. & N.d. & N.d. & N.d. \\
\hline \multicolumn{7}{c}{ Retention time [min]. ** Signal intensity [peak area] as mean s.d. } &
\end{tabular}

\subsection{Metabolic Diversity of Hairy Root Exudates and Genetic Diversity in Hyoscyamus niger}

One of the goals of studies of chemical diversity in plants is to assess the relationship between metabolic and genetic diversity. The results of a limited number of studies indicated that patterns of genetic and metabolic similarity may be incongruent $[34,35,68]$. The bioreactor designed in this work will facilitate comparison of root exudates and/or hairy root exudates for plant accessions with known genetic relationships. To demonstrate this approach on hairy root exudates of Hyoscyamus niger (see Section 3.7.), we determined pairwise Euclidean distances among normalized metabolic profiles of hairy root exudates of three varieties of $H$. niger. To determine the effect of cultivation system on the composition of the exudates, we also determined the distances between metabolic profiles of each variety grown in bioreactors and in shaking flasks. 
The results showed that the differences among accessions were of the same magnitude as differences due to cultivation conditions (Figure 10). To obtain a first insight into the relationship between metabolic profiles and genetic distances among plant accessions from which the hairy roots were derived, the third intron of the Tr2 gene was sequenced. Tr2 encodes tropinone reductase, which is involved in the synthesis of tropane alkaloids. The sequences were deposited in NCBI under Nos. MN017172 (accession Iraq), MN017173 (accession Iran), and MN017174 (accession Germany). The number of nucleotide differences per $1000 \mathrm{bp}$ between the sequences from the accessions Iraq/Iran and Iraq/Germany were 21 and 23, respectively, while the accessions Iran and Germany differed in only 2 nucleotides. Thus the accessions Iran and Germany appeared more closely related than each of them to the accession Iraq. The similarity between sequences of a Tr2 gene of the accessions Iran and Germany was not reflected by the similarities of metabolic profiles of their hairy root exudates (Figure 10). Depending on cultivation conditions, the most similar profiles were Iraq/Germany and Iraq/Iran. The discrepancy between metabolic and genetic similarity is in line with the results of previous studies in other plant species $[34,35,68]$. In contrast, chemical profiles and microsatellite markers in Citrus aurantium were correlated [69]. Hyoscyamus niger is an attractive model to study the relationship between metabolic and genetic diversity in plants because it is a source of medicinally useful tropane alkaloids [70]. Attempts to produce tropane alkaloids in hairy root cultures of Hyoscyamus niger began decades ago [71] yet nothing is known about the genetic variation within the species. The availability of small-scale, inexpensive bioreactors will allow for comprehensive sampling of metabolic diversity in hairy root cultures of $H$. niger, which will facilitate selection of accessions for production purposes and enable studies of the relationship between metabolic and genetic diversity in this medical plant.

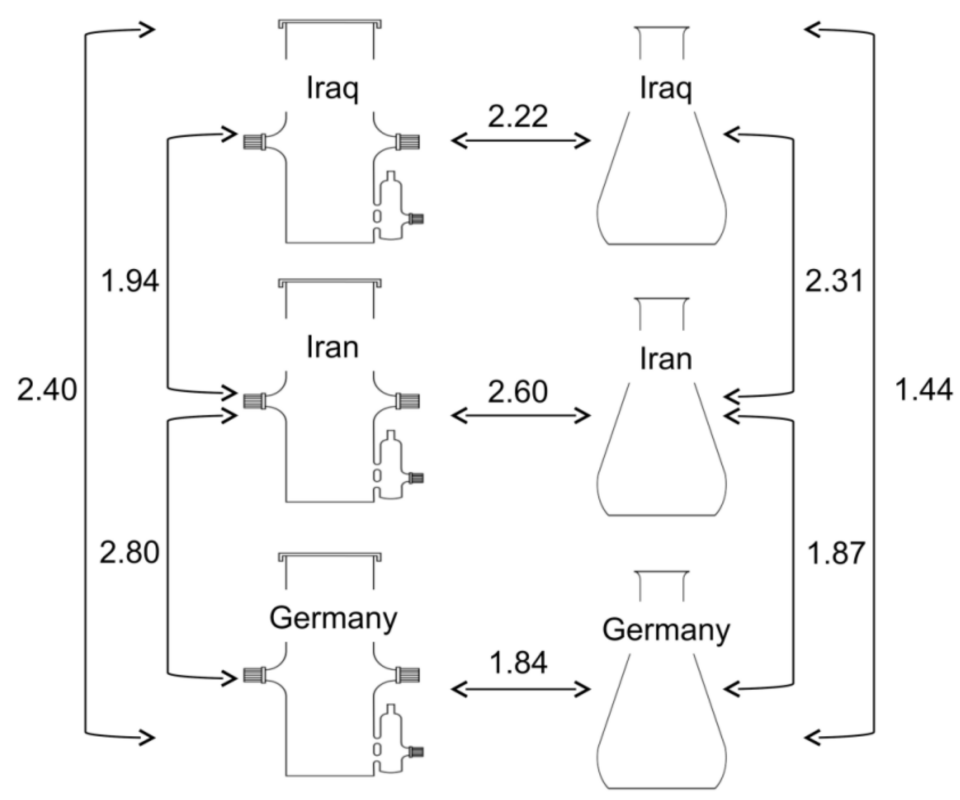

Figure 10. Euclidean distances between normalized metabolic profiles of hairy root exudates obtained from three accessions of Hyoscyamus niger (designated Iraq, Iran and Germany) grown in bioreactors and flask cultures.

\section{Conclusions}

The bioreactor designed in this work proved suitable for growing plants in sterile hydroponics, for cultivating hairy roots, and for collecting root and hairy root exudates. Hairy root cultures accumulated biomass at a comparable rate or faster in the bioreactor than in shaking flasks. The bioreactor outperformed shaking flasks regarding the diversity and quantity of hairy root exudates of $H$. niger.

Validation experiments lead to new findings regarding the metabolic diversity in hairy root exudates of Hyoscyamus niger, which turned out to be incongruent with the genetic relationships among 
plant accessions. Salicylic acid secretion by hairy roots of Hyoscyamus niger varied widely among plant accessions and between cultivation conditions.

Author Contributions: Conceptualization, A.H.A.H.R. and P.K.; Formal analysis, F.H. and P.K.; Funding acquisition, A.H.A.H.R. and P.K.; Investigation, Z.J.K., L.S., A.R., A.S., and F.H.; Methodology, Z.J.K., L.S., A.R., A.S. and F.H.; Resources, A.H.A.H.R. and P.K.; Validation, Z.J.K., L.S. and A.R.; Visualization, Z.J.K., L.S., A.R., F.H., and P.K.; Writing_-original draft, P.K.; Writing—review and editing, P.K.

Funding: This research was funded by the University of Sulaimani, Iraqi Kurdistan Region, and by German Research Foundation (DFG IRTG 2172).

Acknowledgments: The authors are obliged to Hernan Laurentin for providing sesame seeds and Ruth Pilot for technical assistance.

Conflicts of Interest: The authors declare no conflict of interest. The funders had no role in the design of the study; in the collection, analyses, or interpretation of data; in the writing of the manuscript, or in the decision to publish the results.

\section{References}

1. Khorassini, R.; Hettwer, U.; Ratzinger, A.; Steingrobe, B.; Karlovsky, P.; Claassen, N. Citramalic acid and salicylic acid in sugar beet root exudates solubilize soil phosphorus. BMC Plant Biol. 2011, 11, 121. [CrossRef] [PubMed]

2. Tawaraya, K.; Horie, R.; Saito, S.; Wagatsuma, T.; Saito, K.; Oikawa, A. Metabolite profiling of root exudates of common bean under phosphorus deficiency. Metabolites 2014, 4, 599-611. [CrossRef] [PubMed]

3. Zhang, D.; Zhang, C.; Tang, X.; Li, H.; Zhang, F.; Rengel, Z.; Whalley, W.R.; Davies, W.J.; Shen, J. Increased soil phosphorus availability induced by faba bean root exudation stimulates root growth and phosphorus uptake in neighbouring maize. New Phytol. 2016, 209, 823-831. [CrossRef] [PubMed]

4. Hu, L.; Robert, C.A.M.; Cadot, S.; Zhang, X.; Ye, M.; Li, B.; Manzo, D.; Chervet, N.; Steinger, T.; van der Heijden, M.G.A.; et al. Root exudate metabolites drive plant-soil feedbacks on growth and defense by shaping the rhizosphere microbiota. Nature Commun. 2018, 9, 2738. [CrossRef] [PubMed]

5. Watt, M.; Kirkegaard, J.A.; Passioura, J.B. Rhizosphere biology and crop productivity—a review. Soil Res. 2006, 44, 299-317. [CrossRef]

6. Miller, S.B.; Heuberger, A.L.; Broeckling, C.D.; Jahn, C.E. Non-targeted metabolomics reveals sorghum rhizosphere-associated exudates are influenced by the belowground interaction of substrate and sorghum genotype. Int. J. Mol. Sci. 2019, 20, 431. [CrossRef] [PubMed]

7. Huber, M.; Bont, Z.; Fricke, J.; Brillatz, T.; Aziz, Z.; Gershenzon, J.; Erb, M. A below-ground herbivore shapes root defensive chemistry in natural plant populations. Proc. Biol. Sci. 2016, 283, 20160285. [CrossRef] [PubMed]

8. Steinkellner, S.; Lendzemo, V.; Langer, I.; Schweiger, P.; Khaosaad, T.; Toussaint, J.P.; Vierheilig, H. Flavonoids and strigolactones in root exudates as signals in symbiotic and pathogenic plant-fungus interactions. Molecules 2007, 12, 1290-1306. [CrossRef]

9. Lanoue, A.; Burlat, V.; Schurr, U.; Röse, U.S.R. Induced root-secreted phenolic compounds as a belowground plant defense. Plant Signal. Behav. 2010, 5, 1037-1038. [CrossRef]

10. Arafat, Y.; Wei, X.; Jiang, Y.; Chen, T.; Saqib, H.S.A.; Lin, S.; Lin, W. Spatial distribution patterns of root-associated bacterial communities mediated by root exudates in different aged ratooning tea monoculture systems. Int. J. Mol. Sci. 2017, 18, 1727. [CrossRef]

11. Hiltpold, I.; Jaffuel, G.; Turlings, T.C.J. The dual effects of root-cap exudates on nematodes: From quiescence in plant-parasitic nematodes to frenzy in entomopathogenic nematodes. J. Exp. Bot. 2015, 66, 603-611. [CrossRef] [PubMed]

12. Georgiev, M.I.; Agostini, E.; Ludwig-Müller, J.; Xu, J. Genetically transformed roots: From plant disease to biotechnological resource. Trends Biotechnol. 2012, 30, 528-537. [CrossRef] [PubMed]

13. Bulgakov, V.P.; Shkryl, Y.N.; Veremeichik, G.N.; Gorpenchenko, T.Y.; Vereshchagina, Y.V. Recent advances in the understanding of Agrobacterium rhizogenes-derived genes and their effects on stress resistance and plant metabolism. Adv. Biochem. Eng. Biotechnol. 2013, 134, 1-22. [CrossRef] [PubMed] 
14. Yu, H.; Guo, W.; Yang, D.; Hou, Z.; Liang, Z. Transcriptional profiles of SmWRKY family genes and their putative roles in the biosynthesis of tanshinone and phenolic acids in Salvia miltiorrhiza. Int. J. Mol. Sci. 2018, 19, 1593. [CrossRef] [PubMed]

15. Shi, W.Y.; Du, Y.T.; Ma, J.; Min, D.H.; Jin, L.G.; Chen, J.; Chen, M.; Zhou, Y.B.; Ma, Y.Z.; Xu, Z.S.; et al. The WRKY transcription factor GmWRKY12 confers drought and salt tolerance in soybean. Int. J. Mol. Sci. 2018, 19. [CrossRef] [PubMed]

16. Sańko-Sawczenko, I.; Dmitruk, D.; Łotocka, B.; Różańska, E.; Czarnocka, W. Expression analysis of pin genes in root tips and nodules of Lotus japonicus. Int. J. Mol. Sci. 2019, 20, 235. [CrossRef] [PubMed]

17. Bai, Z.; Xia, P.; Wang, R.; Jiao, J.; Ru, M.; Liu, J.; Liang, Z. Molecular cloning and characterization of five SmGRAS genes associated with tanshinone biosynthesis in Salvia miltiorrhiza hairy roots. PLoS ONE 2017, 12, e0185322. [CrossRef] [PubMed]

18. Morriss, S.C.; Studham, M.E.; Tylka, G.L.; MacIntosh, G.C. Validation of a hairy roots system to study soybean-soybean aphid interactions. PLoS ONE 2017, 12, e0174914. [CrossRef]

19. Georgiev, M.I.; Pavlov, A.I.; Bley, T. Hairy root type plant in vitro systems as sources of bioactive substances. Appl. Microbiol. Biotechnol. 2007, 74, 1175-1185. [CrossRef]

20. Kochan, E.; Balcerczak, E.; Szymczyk, P.; Sienkiewicz, M.; Zielińska-Bliźniewska, H.; Szymańska, G. Abscisic acid regulates the 3-hydroxy-3-methylglutaryl CoA reductase gene promoter and ginsenoside production in Panax quinquefolium hairy root cultures. Int. J. Mol. Sci. 2019, 20, 1310. [CrossRef]

21. Renouard, S.; Corbin, C.; Drouet, S.; Medvedec, B.; Doussot, J.; Colas, C.; Maunit, B.; Bhambra, A.S.; Gontier, E.; Jullian, N.; et al. Investigation of Linum flavum (L.) hairy root cultures for the production of anticancer aryltetralin lignans. Int. J. Mol. Sci. 2018, 19, 990. [CrossRef] [PubMed]

22. Srivastava, S.; Srivastava, A.K. Hairy root culture for mass-production of high-value secondary metabolites. Crit. Rev. Biotechnol. 2007, 27, 29-43. [CrossRef]

23. Nakajima, K.; Hashimoto, T. Two tropinone reductases, that catalyze opposite stereospecific reductions in tropane alkaloid biosynthesis, are localized in plant root with different cell-specific patterns. Plant Cell Physiol. 1999, 40, 1099-1107. [CrossRef]

24. Lee, Y.S.; Ku, K.M.; Becker, T.M.; Juvik, J.A. Chemopreventive glucosinolate accumulation in various broccoli and collard tissues: Microfluidic-based targeted transcriptomics for by-product valorization. PLoS ONE 2017, 12, e0185112. [CrossRef]

25. Jouhikainen, K.; Lindgren, L.; Jokelainen, T.; Hiltunen, R.; Teeri, T.H.; Oksman-Caldentey, K.M. Enhancement of scopolamine production in Hyoscyamus muticus L. hairy root cultures by genetic engineering. Planta 1999, 208, 545-551. [CrossRef]

26. Ludwig-Müller, J.; Jahn, L.; Lippert, A.; Püschel, J.; Walter, A. Improvement of hairy root cultures and plants by changing biosynthetic pathways leading to pharmaceutical metabolites: Strategies and applications. Biotechnol. Adv. 2014, 32, 1168-1179. [CrossRef]

27. Guo, Z.; Tan, H.; Lv, Z.; Ji, Q.; Huang, Y.; Liu, J.; Chen, D.; Diao, Y.; Si, J.; Zhang, L. Targeted expression of Vitreoscilla hemoglobin improves the production of tropane alkaloids in Hyoscyamus niger hairy roots. Sci. Rep. 2018, 8, 17969. [CrossRef]

28. Wei, T.; Gao, Y.; Deng, K.; Zhang, L.; Yang, M.; Liu, X.; Qi, C.; Wang, C.; Song, W.; Zhang, Y.; et al. Enhancement of tanshinone production in Salvia miltiorrhiza hairy root cultures by metabolic engineering. Plant Methods 2019, 15, 53. [CrossRef]

29. Kirchner, T.W.; Niehaus, M.; Debener, T.; Schenk, M.K.; Herde, M. Efficient generation of mutations mediated by CRISPR/Cas9 in the hairy root transformation system of Brassica carinata. PLoS ONE 2017, 12, e0185429. [CrossRef]

30. Madeira, L.M.; Szeto, T.H.; Henquet, M.; Raven, N.; Runions, J.; Huddleston, J.; Garrard, I.; Drake, P.M.W.; Ma, J.K.C. High-yield production of a human monoclonal IgG by rhizosecretion in hydroponic tobacco cultures. Plant Biotechnol. J. 2016, 14, 615-624. [CrossRef]

31. Mai, N.T.P.; Boitel-Conti, M.; Guerineau, F. Arabidopsis thaliana hairy roots for the production of heterologous proteins. Plant Cell Tissue Organ Cult. 2016, 127, 489-496. [CrossRef]

32. Häkkinen, S.T.; Raven, N.; Henquet, M.; Laukkanen, M.L.; Anderlei, T.; Pitkänen, J.P.; Twyman, R.M.; Bosch, D.; Oksman-Caldentey, K.M.; Schillberg, S.; et al. Molecular farming in tobacco hairy roots by triggering the secretion of a pharmaceutical antibody. Biotechnol. Bioeng. 2014, 111, 336-346. [CrossRef] 
33. Van Leeuwen, W.; Ruttink, T.; Borst-Vrenssen, A.W.; van der Plas, L.H.; van der Krol, A.R. Characterization of position-induced spatial and temporal regulation of transgene promoter activity in plants. J. Exp. Bot. 2001, 52, 949-959. [CrossRef]

34. Laurentin, H.; Ratzinger, A.; Karlovsky, P. Relationship between metabolic and genomic diversity in sesame (Sesamum indicum L.). BMC Genom. 2008, 9, 250. [CrossRef]

35. Sarrou, E.; Ganopoulos, I.; Xanthopoulou, A.; Masuero, D.; Martens, S.; Madesis, P.; Mavromatis, A.; Chatzopoulou, P. Genetic diversity and metabolic profile of Salvia officinalis populations: Implications for advanced breeding strategies. Planta 2017, 246, 201-215. [CrossRef] [PubMed]

36. Watanabe, S.; Ohtani, Y.; Aoki, W.; Uno, Y.; Sukekiyo, Y.; Kubokawa, S.; Ueda, M. Detection of betacyanin in red-tube spinach (Spinacia oleracea) and its biofortification by strategic hydroponics. PLoS ONE 2018, 13, e0203656. [CrossRef]

37. Kittipongpatana, N.; Hock, R.S.; Porter, J.R. Production of solasodine by hairy root, callus, and cell suspension cultures of Solanum aviculare Forst. Plant Cell Tissue Organ Cult. 1998, 52, 133-143. [CrossRef]

38. Palavalli, R.R.; Srivastava, S.; Srivastava, A.K. Development of a mathematical model for growth and oxygen transfer in in vitro plant hairy root cultivations. Appl. Biochem. Biotechnol. 2012, 167, 1831-1844. [CrossRef]

39. Georgiev, M.I.; Eibl, R.; Zhong, J.J. Hosting the plant cells in vitro: Recent trends in bioreactors. Appl. Microbiol. Biotechnol. 2013, 97, 3787-3800. [CrossRef]

40. Homova, V.; Weber, J.; Schulze, J.; Alipieva, K.; Bley, T.; Georgiev, M. Devil's claw hairy root culture in flasks and in a 3-L bioreactor: Bioactive metabolite accumulation and flow cytometry. Z. Nat. C J. Biosci. 2010, 65, 472-478. [CrossRef]

41. Patra, N.; Srivastava, A.K. Enhanced production of artemisinin by hairy root cultivation of Artemisia annua in a modified stirred tank reactor. Appl. Biochem. Biotechnol. 2014, 174, 2209-2222. [CrossRef]

42. Medina-Bolívar, F.; Cramer, C. Production of recombinant proteins by hairy roots cultured in plastic sleeve bioreactors. Methods Mol. Biol. 2004, 267, 351-363. [CrossRef]

43. Kwok, K.H.; Doran, P.M. Kinetic and stoichiometric analysis of hairy roots in a segmented bubble column reactor. Biotechnol. Prog. 1995, 11, 429-435. [CrossRef]

44. Weathers, P.J.; Giles, K.L. Regeneration of plants using nutrient mist culture. Vitr. Cell. Dev. Biol. 1988, 24, 727-732. [CrossRef]

45. Tscheschke, B.; Dreimann, J.; von der Ruhr, J.W.; Schmidt, T.; Stahl, F.; Just, L.; Scheper, T. Evaluation of a new mist-chamber bioreactor for biotechnological applications. Biotechnol. Bioeng. 2015, 112, 1155-1164. [CrossRef]

46. Caspeta, L.; Quintero, R.; Villarreal, M.L. Novel airlift reactor fitting for hairy root cultures: Developmental and performance studies. Biotechnol. Prog. 2005, 21, 735-740. [CrossRef]

47. Williams, G.R.; Doran, P.M. Hairy root culture in a liquid-dispersed bioreactor: Characterization of spatial heterogeneity. Biotechnol. Prog. 2000, 16, 391-401. [CrossRef]

48. Higuchi, M.; Kunitomo, N.; Kobayashi, Y.; Kumada, K.; Tanaka, N. Bioreactor using oxygen-enriched micro/nano-bubbles. International Patent Application No. PCT/JP2015/071597, 2 February 2017.

49. Khuntia, S.; Majumder, S.K.; Ghosh, P. Microbubble-aided water and wastewater purification: A review. Rev. Chem. Eng. 2012, 28, 191-221. [CrossRef]

50. Al-Mashhadani, M.K.H.; Wilkinson, S.J.; Zimmerman, W.B. Airlift bioreactor for biological applications with microbubble mediated transport processes. Chem. Eng. Sci. 2015, 137, 243-253. [CrossRef]

51. Mochida, K.; Furuta, T.; Ebana, K.; Shinozaki, K.; Kikuchi, J. Correlation exploration of metabolic and genomic diversity in rice. BMC Genom. 2009, 10, 568. [CrossRef]

52. Moore, B.D.; Andrew, R.L.; Külheim, C.; Foley, W.J. Explaining intraspecific diversity in plant secondary metabolites in an ecological context. New Phytol. 2014, 201, 733-750. [CrossRef]

53. Rengel, Z. Genetic control of root exudation. Plant Soil 2002, 245, 59-70. [CrossRef]

54. Klessig, D.F.; Malamy, J. The salicylic acid signal in plants. Plant Mol. Biol. 1994, 26, 1439-1458. [CrossRef]

55. Ratzinger, A.; Riediger, N.; von Tiedemann, A.; Karlovsky, P. Salicylic acid and salicylic acid glucoside in xylem sap of Brassica napus infected with Verticillium longisporum. J. Plant Res. 2009, 122, 571-579. [CrossRef]

56. Lebeis, S.L.; Paredes, S.H.; Lundberg, D.S.; Breakfield, N.; Gehring, J.; McDonald, M.; Malfatti, S.; Glavina del Rio, T.; Jones, C.D.; Tringe, S.G.; et al. Salicylic acid modulates colonization of the root microbiome by specific bacterial taxa. Science 2015, 349, 860-864. [CrossRef] 
57. Sirrenberg, A.; Nutz, S.; Ratzinger, A.; Karlovsky, P. Replant disease of roses-new research approaches. Gartenpraxis 2009, 9, 16-20.

58. Zedníková, M.; Orvalho, S.; Fialová, M.; Ruzicka, M.C. Measurement of volumetric mass transfer coefficient in bubble columns. ChemEngineering 2018, 2, 19. [CrossRef]

59. Vervliet, G.; Holsters, M.; Teuchy, H.; Van Montagu, M.; Schell, J. Characterization of different plaque-forming and defective temperate phages in Agrobacterium. J. Gen. Virol. 1975, 26, 33-48. [CrossRef]

60. Murashige, T.; Skoog, F. A revised medium for rapid growth and bioassays with tobacco tissue culture. Physiol. Plant. 1962, 15, 473-497. [CrossRef]

61. Kumar, S.; Stecher, G.; Li, M.; Knyaz, C.; Tamura, K. MEGA X: Molecular evolutionary genetics analysis across computing platforms. Mol. Biol. Evol. 2018, 35, 1547-1549. [CrossRef]

62. Hand, D.W. Effects of atmospheric humidity on greenhouse crops. Acta Hortic. 1988, 229, 143-158. [CrossRef]

63. Cámara-Zapata, J.M.; Sánchez-Molina, J.A.; Rodríguez, F.; López, J.C. Evaluation of a dehumidifier in a mild weather greenhouse. Appl. Therm. Eng. 2019, 146, 92-103. [CrossRef]

64. Ramirez-Estrada, K.; Vidal-Limon, H.; Hidalgo, D.; Moyano, E.; Golenioswki, M.; Cusidó, R.M.; Palazon, J. Elicitation, an effective strategy for the biotechnological production of bioactive high-added value compounds in plant cell factories. Molecules 2016, 21, 182. [CrossRef]

65. Kang, S.M.; Jung, H.Y.; Kang, Y.M.; Yun, D.J.; Bahk, J.D.; Yang, J.; Choi, M.S. Effects of methyl jasmonate and salicylic acid on the production of tropane alkaloids and the expression of PMT and $\mathrm{H} 6 \mathrm{H}$ in adventitious root cultures of Scopolia parviflora. Plant Sci. 2004, 166, 745-751. [CrossRef]

66. Harfi, B.; Khelifi, L.; Khelifi-Slaoui, M.; Assaf-Ducrocq, C.; Gontier, E. Tropane alkaloids GC/MS analysis and low dose elicitors' effects on hyoscyamine biosynthetic pathway in hairy roots of Algerian Datura species. Sci. Rep. 2018, 8, 17951. [CrossRef]

67. Adnani, N.; Michel, C.R.; Bugni, T.S. Universal quantification of structurally diverse natural products using an evaporative light scattering detector. J. Nat. Prod. 2012, 75, 802-806. [CrossRef]

68. Houshyani, B.; Kabouw, P.; Muth, D.; de Vos, R.C.H.; Bino, R.J.; Bouwmeester, H.J. Characterization of the natural variation in Arabidopsis thaliana metabolome by the analysis of metabolic distance. Metabolomics 2012, 8, 131-145. [CrossRef]

69. Lamine, M.; Rahali, F.Z.; Hamdaoui, G.; Selmi, S.; Mliki, A.; Gargouri, M. Associating chemical analysis to molecular markers for the valorization of Citrus aurantium leaves: A useful starting point for marker-assisted selection. Euphytica 2017, 213, 44. [CrossRef]

70. Grynkiewicz, G.; Gadzikowska, M. Tropane alkaloids as medicinally useful natural products and their synthetic derivatives as new drugs. Pharmacol. Rep. 2008, 60, 439-463.

71. Hashimoto, T.; Yukimune, Y.; Yamada, Y. Tropane alkaloid production in Hyoscyamus root cultures. J. Plant Physiol. 1986, 124, 61-75. [CrossRef] 


\section{Acknowledgements}

My deepest gratitude goes first and foremost to my supervisor Prof. Dr. Petr Karlovsky for giving me this opportunity to do my $\mathrm{PhD}$ in the doctor program IRTG 2172 PRoTECT and supervising me. I learned a lot from him, not only research skills, but also life experience. I benefit from it all through my life. I want to thank Prof. Dr. Ivo Feussner for being a member of my thesis committee. I am thankful to our cooperation partner and member of the thesis advisory committee Prof. Yuelin Zhang for his commitment, and hospitality during my stay in his group.

Additionally, I thank Prof. Dr. Gerhard Braus, Prof. Dr. Christiane Gatz and Prof. Dr. Andrea Polle for being my members of examination board. Many thanks to all the members of PRoTECT for fruitful discussions in frame of our meeting. I thank DFG for supporting me the cost in Canada and for conferences. My sincere thanks go to all the students of PRoTECT for their kind help and friendship.

I am very lucky that I am a member of Lab Karlovsky. It is not only a place where I did my research and receive my degree, but also a big warm family. I am thankful to all the members of Lab Karlovsky. I thank Yang Xu for our good cooperation in Aurofusarin project, and Dr. Maria Vinas for her guide me in project management. Every time I were under high time pressure, Heike Rollwage always help me doing experiments as much as she can. Ruth Pilot guided and supported me in molecular assay a lot. I can imagine it would be much harder to perform experiments without her help. Besides, many thanks to Ruth for helping me produced and purified VINLP1 protein. I very appreciate that Nahid Najafi Hajiwar put a lot of efforts on phytoalexins synthesis. Many thanks to Luciana Macis who helped me extracting samples from plants. I thank Dr. Anna Rathgeb for helping all the HPLC experiments. Many thanks to Lukas Beule for giving me good suggestions. I thank Isabella Reitz and Chelsea Schreiber for helping me doing experiments. My thanks also goes to Makrina Diakaki, Albatol Alsarrag, Rasoul Abousaeedi, Dr. Mohammad Alhussein, Simon Schiwek, and Ana Flores.

Further, the PRoTECT made the cooperation with the University of British Columbia possible to get international experience and build up an international network. I had the chance to organize the invitation and talk of a great group leader from Bayer CropScience, from which I learnt a lot for my future career. 
My acknowledgements go to Prof. Andreas von Tiedemann group for providing me all fungal strains. More important, I obtained a lot of suggestions and supports from Dr. Xiaorong Zheng. She taught me the plants experiments and assist me in my thesis. Besides, I want to thanks Marta Vega Marin for providing isolates.

My gratitude further goes to Zhang lab.in Vancouver who hosted me during my stay. Many thanks to Dr. Tongjun Sun and Dr. Qian Sun for guiding me all the experiments. A special thanks to Dr. Yuli Ding for supporting me a lot in Vancouver. I also want to thank Yujun Peng, Di Wu, Weijie Huang and all members.

I would like to thank Dr. Gerhard Braus for providing me the chance to use data from VertiBase. Many thanks to Dr. Tri-Thuc Bui for providing me pGreen2 plasmid.

For the proofreading of my thesis, I would like to thank Dr. Franz Hadacek and Dr. Xiaorong. Zheng. Dr. Maria Vinas, Wanying He and Dr. Anna Rathgeb also gave me valuable suggestions. Prof. Karlovsky gave me critical comments and suggestions, especially for summaries.

Last but not least, I owe my thanks to my beloved parents and friends. Because of them, I was able to motivate when I felt dispirited or faced to difficult time, and had an unforgettable happy life in Göttingen. 